\title{
Macromolecular Engineering of In-situ Forming Hydrogels
}

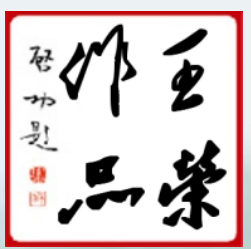




\section{MACROMOLECULAR ENGINEERING OF IN-SITU FORMING HYDROGELS}

Rong Wang

2016 


\section{Members of graduation committee}

\section{Chairman:}

Prof.dr.ir. J.W.M. Hilgenkamp

University of Twente

Promoters:

Prof.dr. H.B.J. Karperien

University of Twente

Prof.dr. P.J.Dijkstra

University of Twente

\section{Members:}

Prof.dr.ir. W.E. Hennink University of Utrecht

Dr. T.H. van Kuppevelt Radboud University Medical Center

Prof.dr.ir. L.H. Koole Interface BIOmaterials BV

Prof.dr. J. Feijen

University of Twente

Prof.dr. ir. J. Huskens

University of Twente

Prof.dr. D.B.F. Saris

University of Twente

Prof.dr. P.C.J.J. Passier

University of Twente

\section{NIRM UNIVERSITY OF TWENTE. MIIA}

Macromolecular engineering of in-situ forming hydrogels

Jan., 2016, Rong Wang r.wang@utwente.nl

This research was performed in department of Developmental Bioengineering (DBE), Faculty of Science and Technology (TNW), University of Twente, P.O. Box 217, 7500 AE Enschede, The Netherlands.

The printing of this thesis was kindly supported by the Netherlands Society of Biomaterials and Tissue Engineering (NBTE).

ISBN: 978-90-365-4023-0

DOI: $10.3990 / 1.9789036540230$

Printed by: Gildeprint, Enschede 


\title{
MACROMOLECULAR ENGINEERING OF IN-SITU FORMING HYDROGELS
}

\author{
DISSERTATION \\ To obtain \\ the degree of doctor at the University of Twente, \\ on the authority of the rector magnificus, \\ Prof.dr. H. Brinksma, \\ on account of the decision of the graduation committee, \\ to be publicly defended \\ on Thursday, $21^{\text {st }}$ of January, 2016 at 14.45 hrs
}

by

\section{Rong Wang}

born on the $1^{\text {st }}$ of February, 1984

in Xuzhou, P.R. China 
This dissertation has been approved by:

Promotor: Prof.dr. D.J. H.B. Karperien

Promotor: Prof.dr. P.J. Dijkstra

(C) Rong Wang 2016

All rights reserved. 
Dedicated to my beloved family my dear supervisor Prof.dr. Piet Dijkstra 



\section{Contents}

Chapter 1 General introduction 1

Chapter 2 Naturally and synthetically derived biomedical materials: A literature review.

Chapter 3 Cartilage adhesive and mechanical properties of enzymatically crosslinked polysaccharide tyramine conjugate hydrogels 39

Chapter 4 Non-invasive near-infrared fluorescence in-vivo imaging of biodegradable enzymatically crosslinked polysaccharide hydrogels 55

Chapter 5 Enzymatic co-crosslinking of star-shaped poly(ethylene glycol) tyramine and hyaluronic acid tyramine conjugates provides elastic biocompatible and biodegradable hydrogels

Chapter 6 Hydrogels by supramolecular crosslinking of terpyridine end group functionalized 8 arm poly(ethylene glycol)

Chapter 7 Kinetically stable metal ligand charge transfer complexes as crosslinks in nanogels/hydrogels: physical properties and cytotoxicity

Summary 165

Samenvatting 169

摘 要 173

Acknowledgements 175

About the author 178

Publications 179 



\section{Chapter 1}

\section{General Introduction}

\subsection{Minimally invasive surgery and articular cartilage damage}

Surgery in our perspective generally requires an operation with a certain size of an incision, leaving wounds, which are painful, require a long time to heal and end up with the formation of scars. Advances in medical procedures and technologies have led to the development of minimally invasive surgical procedures and several imaging tools for diagnosis. Imaging by techniques like e.g. Magnetic Resonance Imaging (MRI) has become increasingly important since it provides 3D-information in a non-invasive manner [1]. If possible, in follow-up surgery, a minimally invasive procedure with a small incision is applied because of the time reduction for a wound to heal and of prime importance, to diminish the risk of infection [2].

For articulating joints like the knee, a typical procedure to diagnose and treat cartilage tissue or replace torn ligaments is the use of arthroscopic techniques [3]. It uses an endoscope (arthroscope) through which surgical instruments can be inserted and a fiber optic camera to view real time procedures at the location site. The arthroscope is inserted through a small incision in the skin and the surgical procedure is followed on a monitor connected to the camera.

Articular cartilage damage can originate from a variety of causes, like an accident or traumatic sport-accidents. Such knee injuries, especially when not recognized by the patient, can cause the wear of cartilage over time. Osteoarthritis (OA), a degenerative joint disease will develop and results in irreversible loss of structure and function of the tissue [4]. Articular cartilage damage therefore strongly affects a person's quality of life because it causes pain, substantial reduction in mobility and severe restrictions to activities. Replacement of the joint with an artificial knee is the end stage for a patient. Articular cartilage cannot self-regenerate after injury or disease because of its aneural and 
avascular nature, spare cell population and low mitotic activity of cells, the chondrocytes [5]. Therefore, treatment at an early stage is critical to prevent the progression of cartilage deterioration. Tissue engineering approaches are currently recognized to solve this problem by applying a temporary construct filling the defect and stimulating surrounding healthy cells to migrate and produce new tissue extra cellular matrix at the defect site [68].

\subsection{Historical perspective and developmental bioengineering}

About 10 years ago the Dutch Program for Tissue Engineering (DPTE) started as a consortium of Dutch Universities, Academic hospitals, knowledge institutions and industries. A large number of projects were financed and one of these projects was directed to the development of enzymatically cross-linked hydrogels and another one to cartilage regeneration. The enzymatically cross-linked hydrogels were based on earlier work of Kaplan who showed that water soluble poly(r-glutamic acid) conjugated with phenolic groups afforded a hydrogel by crosslinking using the enzyme horseradish peroxidase in the presence of hydrogen peroxide [9]. This method was a few years later picked up by Kurisawa who showed that similar conjugates of hyaluronic acid, an important polysaccharide in cartilage, was cross-linked into a hydrogel in a similar way [10]. The plant enzyme, horseradish peroxidase (HRP), couples the phenolic groups covalently via a radical reaction in the presence of hydrogen peroxide which was converted into water molecules. Within the DPTE program the early strategies were directed to crosslink tyramine conjugated of the polysaccharide dextran. Dextran is biocompatible and is used in various biomedical applications, such as a blood expander. It is slowly degraded in the body or excreted. First results on hydrogels prepared from these dextran conjugates revealed excellent mechanical properties and elasticity. Because cartilage is an elastic and very resilient material and has a high water content a link between the two projects on hydrogels and cartilage regeneration was easily made. In first experiments, cartilage cells and the hydrogel were mixed and cultured in vitro. These constructs showed that cells survived well in the watery gel matrix and produced a new extra cellular matrix (ECM) in their periphery with characteristics resembling those of the natural cartilage matrix. These results led to an expansion of this hydrogel technology 
to other polysaccharides using enzymatic crosslinking [11]. Because the hydrogel has only a temporary function it would be desirable that the material degrades in time. The degradation rate should match the secretion rate of newly formed ECM by the encapsulated cells. Using natural polymers like alginate, chitosan, hyaluronic acid and heparin and proteins like collagen, and fibrin, research showed that degradation of these hydrogels in time takes place and the hydrogel was replaced by newly formed tissue. Research eventually focused on hydrogels prepared from a combination of dextran, because of the already established good mechanical properties, and hyaluronic acid, because of its naturally occurrence in cartilage and interaction with cartilage cells; the chondrocytes $[12,13]$.

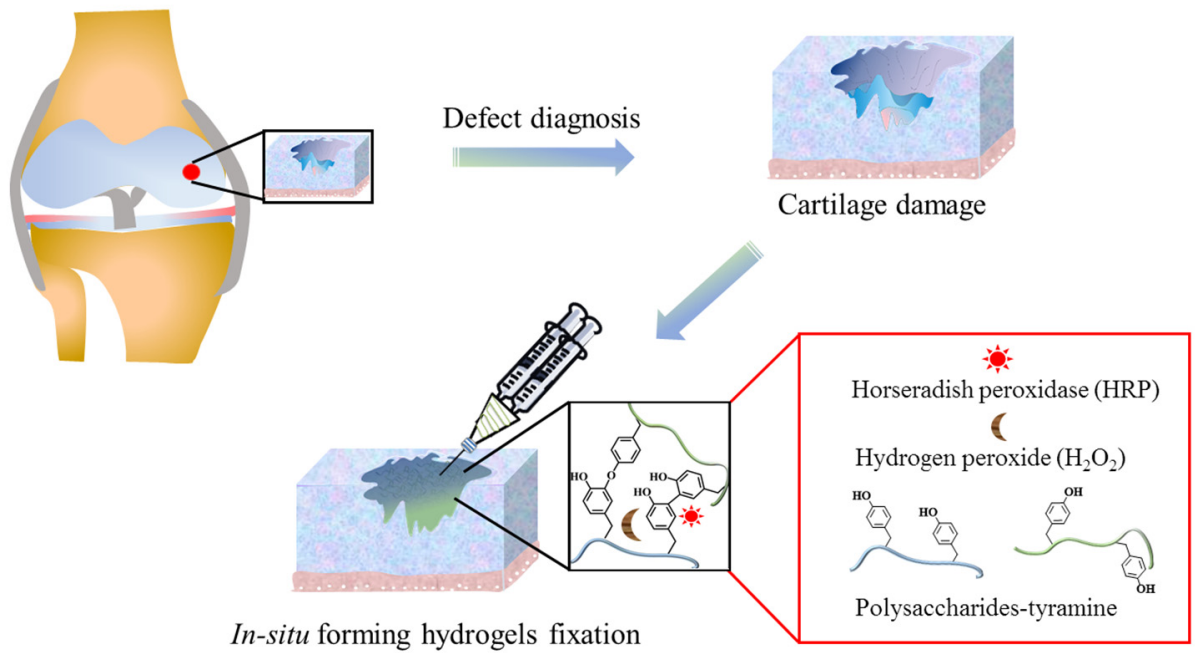

Figure 1.1 Schematic overview of injecting hydrogels to cartilage defect using double syringe system.

The kinetics of the crosslinking of the polysaccharide conjugates was particularly relevant for the design of an in-situ forming (injectable) hydrogel. A two chamber syringe (Mixpac) was chosen for this system and a general scheme of the procedure is presented in Figure 1.1. Both chambers were filled with the mixed polysaccharide solution, but one was mixed with the enzyme and the other was mixed with hydrogen peroxide. The chambers were connected to a mixing chamber. Simultaneous ejection of the solutions gave adequate mixing in the mixing chamber and consecutive formation of the initially 
flowing solution into a hydrogel within a few seconds. First tests were also performed on cartilage defects in bovine knee joints obtained from a local slaughterhouse and revealed proper filling of defects and first indication of good adhesion to the surrounding tissue. Also it was possible to mix chondrocytes into the polymer solution with the enzyme and homogeneous gel-cell constructs were prepared in this way. However, could this technology be translated to a clinically relevant product for filling cartilage defects and stimulate the regeneration of damaged cartilage?

It was realized that still major steps were necessary and in a follow up program, the Netherlands Initiative for Regenerative Medicine (NIRM), further steps were taken. Next to the research on the MIRA labs towards the properties of hydrogels and cartilage formation, initiatives were taken to integrate the application of the in-situ forming hydrogels in an arthroscopic procedure enabling minimally invasive surgery. Veterinarians in a clinic in Den Ham in the Twente area, with facilities for operation techniques on small and large animals, were contacted and they were enthusiastic on collaboration. Facilities were accessible for performing such arthroscopic procedures on horse cadavers. A team of researchers amongst which, an Erasmus student from Germany, a student from Saxion College, a student from Technical Medicine, and staff members of the Developmental BioEngineering group headed for this first clinical horse research.

Two veterinary orthopedic surgeons and staff members from the clinic made a set up and performed the first experiments (Figure 1.2). The surgeons made a small incision in a joint of the horse for inserting the arthroscope. After draining and flushing of the joint, defects were made in the cartilage. Using a fiber optic camera the procedure could be followed on a monitor. After preparation of the double syringe the defects were filled by injection through the skin. Adjusting the final formulation allowed a proper filling of defects. All team members were enthusiastic on these results and convinced this was an excellent basis in the development of the technology towards a clinical application. Currently this technology is transferred to a spin-off company, Hy2Care, which name is derived from Hydrogels, 2 components and Cartilage Regeneration. The opportunities to cooperate with orthopedic surgeons and the early development of the technology were an exciting and a stimulating factor in ongoing research. Despite the considerable amount of time necessary for further product development, which is not taken up in this thesis, new insights in the technology of injectable hydrogels have surfaced. Especially in chapters 3, 
4 and 5 this research work can be recognized.

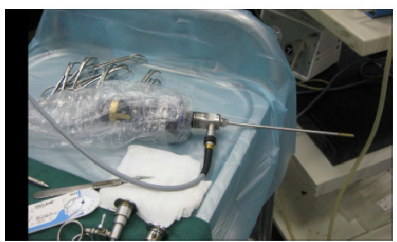

I

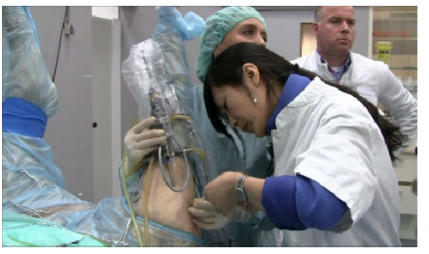

IV

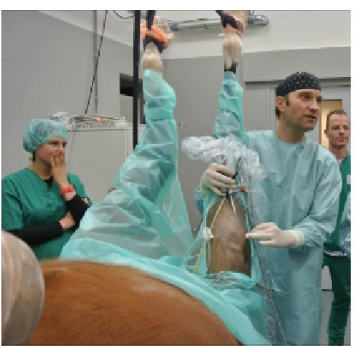

II

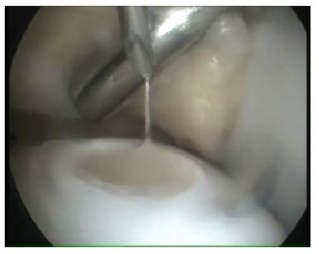

V

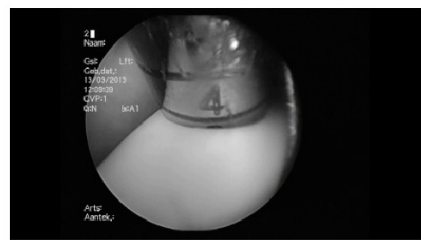

III

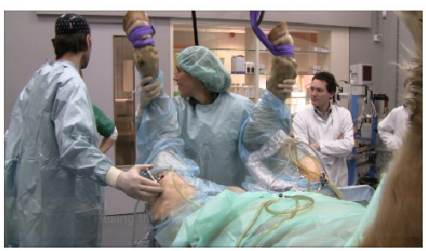

VI

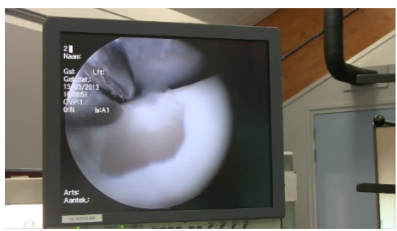

VII

Figure 1.2 Illustration of a minimally invasive procedure on a horse cadaver for filling articular cartilage defects. I: arthroscope, II: insertion and examining cartilage by arthroscopy, III: preparation of a cartilage defect, IV: positioning the double syringe, V: placing the in-situ forming hydrogel, VI: flexing joints and VII: checking gel adhesion.

\subsection{Aim and outline of this thesis}

The research described in this thesis was mainly focused on macromolecular engineering of injectable hydrogels tailored for the treatment of articular cartilage defects. In-situ forming hydrogels, based on the horseradish peroxidase catalyzed crosslinking via the oxidative coupling of phenolic residues in the presence of $\mathrm{H}_{2} \mathrm{O}_{2}$ were studied for their adhesion to tissue and degradation in vivo. Novel hydrogels comprising hyaluronic acid 
and a synthetic eight arm poly(ethylene glycol) were designed to easier control hydrogel properties. In the second part of this thesis, hydrogel formation by supramolecular complexation of ligands by transition metal ions, to provide a novel method for in-situ forming hydrogels, was explored.

In Chapter $\mathbf{2}$ a general overview is presented on the natural polymers dextran and the synthetic polymer 8 arm poly(ethylene glycol) as materials for in-situ forming hydrogels. The review summarizes recent developments based on these polymers in the (bio)medical and pharmaceutical fields.

Articular cartilage is an anisotropic load-bearing tissue, continuously subjected to compressive and shear stresses. The injected hydrogel should provide sufficient mechanical support and transfer of forces. One of the main challenges in the use of hydrogels as articular cartilage scaffolds is their integration and adhesion to native cartilage. An optimal integration method would result in tightly bonding with tissue, be quickly and easily applied clinically, demonstrate biocompatibility and should promote tissue repair. Several approaches have been used to address this major issue, such as incorporation of fiber meshes and sutures or build up a bridge between biomaterials and tissue proteins [14]. In Chapter 3 we introduce a new method to analyze the integration between injectable hydrogels to native tissue. A home built tensile tester was used to evaluate the adhesion and mechanical properties of in-situ forming hydrogels based on dextran-tyramine and hyaluronic acid tyramine conjugates.

Strategies developed for monitoring the in vitro degradation of hydrogels are well known, such as bulk weight loss measurements [11]. Methods for monitoring real-time in vivo hydrogel degradation are magnetic resonance imaging (MRI) and fluorescence spectroscopy. However, a few drawbacks cannot be ignored for MRI imaging, it requires magnetically active materials as contrast agents and sophisticated instrumentation. Near infrared fluorescence imaging remains the most widely used method for tracking materials in vivo at least in small animal models [15-18]. In Chapter 4 we describe a noninvasive method for real time monitoring in vivo degradation of dextrantyramine/hyaluronic acid tyramine conjugate hydrogels by using near infrared fluorescence spectroscopy. The near infrared label was covalently bonded to a dextrantyramine-butyl amine conjugate. The solubility and physical and mechanical properties of the hydrogels were evaluated. The decrease in fluorescence during a 10 weeks 
implantation was followed by imaging.

The advantage of the use of synthetic water soluble polymers compared to natural polymers is their defined structure with no batch to batch variation. End group modification of an 8 arm poly(ethylene glycol) with tyramine groups and enzymatic crosslinking of this polymer with hyaluronic acid-tyramine was studied (Chapter 5). Mechanical properties and biological properties were analyzed to show the potential of these materials as a supportive environment for chondrocyte proliferation and differentiation, as well as matrix deposition.

From coordination chemistry it is known that the bonds between terpyridines and transition metal ions like $\mathrm{Fe}^{2+}$ can be almost as strong as covalent bonds. In Chapter 6 we describe in-situ forming hydrogels by metallo supramolecular assemblies of terpyridine end-groups modified 8 arm poly(ethylene glycol) with the transition metal ions $\mathrm{Ni}^{2+}, \mathrm{Fe}^{2+}, \mathrm{Co}^{2+}$ and $\mathrm{Zn}^{2+}$. The dissociation rate constant of the metal ligand complexes, $\mathrm{Fe}^{2+}$ and $\mathrm{Co}^{2+}$, were for the first time measured in an aqueous environment. Elastic hydrogels were obtained with $\mathrm{Ni}^{2+}, \mathrm{Fe}^{2+}$ and $\mathrm{Co}^{2+}$, whereas a thermoreversible $\mathrm{Zn}^{2+}$ gel was formed.

Cytocompatability studies on supramolecular complexed hydrogels based on complexes of the materials described in Chapter 6 with $\mathrm{Fe}^{2+}$ are presented in Chapter 7 . Cytotoxicity of free ligands and $\mathrm{Fe}^{2+}$ complexes in direct or indirect contact with chondrocytes were determined. We concluded that materials based on kinetically stable metal-ligand interactions may be used in the design, but complexes, which are in thermodynamic equilibrium with the uncomplexed state, are not suitable as biomaterials.

\section{References}

[1] Paul E. K.; Manbir S., Functional magnetic resonance imaging for brain mapping in neurosurgery. Neurosurg. Focus 2003, 15 (1), 1-7.

[2] Sethi, R. K. V.; Henry, A. J.; Hevelone, N. D.; Lipsitz, S. R.; Belkin, M.; Nguyen, L. L., Impact of hospital market competition on endovascular aneurysm repair adoption and outcomes. J. Vasc. Surg. 2013, 58 (3), 596-606. 
[3] Kaufman, J. A.; Reekers, J. A.; Burnes, J. P.; Al-Kutoubi, A.; Lewis, C. A.; Hardy, B. W.; Kuribayashi, S., Global statement defining interventional radiology. J. Vasc. Interv. Radiol. 2010, 21 (8), 1147-1149.

[4] Altman, R.; Asch, E.; Bloch, D.; Bole, G.; Borenstein, D.; Brandt, K.; Christy, W.; Cooke, T. D.; Greenwald, R.; Hochberg, M.; Howell, D.; Kaplan, D.; Koopman, W.; Longley, S.; Mankin, H.; McShane, D. J.; Medsger, T.; Meenan, R.; Mikkelsen, W.; Moskowitz, R.; Murphy, W.; Rothschild, B.; Segal, M.; Sokoloff, L.; Wolfe, F., Development of criteria for the classification and reporting of osteoarthritis classification of osteoarthritis of the knee. Arthritis Rheum. 1986, 29 (8), 1039-1049.

[5] Wang, Y.; Ding, C.; Wluka, A. E.; Davis, S.; Ebeling, P. R.; Jones, G.; Cicuttini, F. M., Factors affecting progression of knee cartilage defects in normal subjects over 2 years. Rheumatology 2006, 45 (1), 79-84.

[6] Griffith, L. G.; Naughton, G., Tissue engineering--current challenges and expanding opportunities. Science 2002, 295 (5557), 1009-1014.

[7] Peretti, G. M.; Campo-Ruiz, V.; Gonzalez, S.; Randolph, M. A.; Xu, J. W.; Morse, K. R.; Roses, R. E.; Yaremchuk, M. J., Tissue engineered cartilage integration to live and devitalized cartilage: A study by reflectance mode confocal microscopy and standard histology. Connect. Tissue Res. 2006, 47 (4), 190-199.

[8] Teixeira, L. S. M.; Bijl, S.; Pully, V. V.; Otto, C.; Rong, J.; Feijen, J.; van Blitterswijk, C. A.; Dijkstra, P. J.; Karperien, M., Self-attaching and cell-attracting in-situ forming dextran-tyramine conjugates hydrogels for arthroscopic cartilage repair. Biomaterials 2012, 33 (11), 3164-3174.

[9] Sofia, S. J.; Singh, A.; Kaplan, D. L., Peroxidase-catalyzed crosslinking of functionalized polyaspartic acid polymers. J Macromol Sci Pure 2002, A39 (10), 11511181.

[10] Lee, F.; Chung, J. E.; Kurisawa, M., An injectable enzymatically crosslinked hyaluronic acid-tyramine hydrogel system with independent tuning of mechanical strength and gelation rate. Soft Matter 2008, 4 (4), 880-887.

[11] Jin, R.; Hiemstra, C.; Zhong, Z. Y.; Feijen, J., Enzyme-mediated fast in situ formation of hydrogels from dextran-tyramine conjugates. Biomaterials 2007, 28 (18), 2791-2800. 
[12] Jin, R.; Teixeira, L. S. M.; Dijkstra, P. J.; van Blitterswijk, C. A.; Karperien, M.; Feijen, J., Enzymatically-crosslinked injectable hydrogels based on biomimetic dextranhyaluronic acid conjugates for cartilage tissue engineering. Biomaterials 2010, 31 (11), 3103-3113.

[13] Wennink, J. W. H.; Niederer, K.; Bochynska, A. I.; Teixeira, L. S. M.; Karperien, M.; Feijen, J.; Dijkstra, P. J. Injectable hydrogels by enzymatic co-crosslinking of dextran and hyaluronic acid tyramine conjugates; Adv. Polym. Med., 2011; Vol. 309-310.

[14] Wang, D. A.; Varghese, S.; Sharma, B.; Strehin, I.; Fermanian, S.; Gorham, J.; Fairbrother, D. H.; Cascio, B.; Elisseeff, J. H., Multifunctional chondroitin sulphate for cartilage tissue-biomaterial integration. Nat Mater 2007, 6 (5), 385-392.

[15] Tanaka, E.; Choi, H. S.; Fujii, H.; Bawendi, M. G.; Frangioni, J. V., Image-guided oncologic surgery using invisible light: Completed pre-clinical development for sentinel lymph node mapping. Ann. Surg. Oncol. 2006, 13 (12), 1671-1681.

[16] Choi, H. S.; Nasr, K.; Alyabyev, S.; Feith, D.; Lee, J. H.; Kim, S. H.; Ashitate, Y.; Hyun, H.; Patonay, G.; Strekowski, L.; Henary, M.; Frangioni, J. V., Synthesis and in vivo fate of zwitterionic near-infrared fluorophores. Angew. Chem. Int. Ed. Engl. 2011, $50(28), 6258-6263$.

[17] Wang, W. W.; Liu, J. J.; Li, C.; Zhang, J.; Liu, J. F.; Dong, A. J.; Kong, D. L., Realtime and non-invasive fluorescence tracking of in vivo degradation of the thermosensitive PEGlyated polyester hydrogel. J Mater Chem B 2014, 2 (26), 4185-4192.

[18] Kim, S. H.; Lee, J. H.; Hyun, H.; Ashitate, Y.; Park, G.; Robichaud, K.; Lunsford,

E.; Lee, S. J.; Khang, G.; Choi, H. S., Near-Infrared Fluorescence Imaging for Noninvasive Trafficking of Scaffold Degradation. Sci. Rep. 2013, 3 (1198), 1-7. 


\section{Chapter 2}

\section{Naturally and synthetically derived biomedical materials: A literature review}

Biomaterials have played a tremendous role in the development of medical devices, drug delivery systems or tissue engineering [1-5]. They have been defined as substances other than foods or drugs contained in therapeutic or diagnostic systems, as well as materials composed of biologically derived components irrespective of their applications [6]. In the middle of the nineteenth century a first generation of materials based on wood, glass or metals were used as implant materials and prosthetic devices $[1,2,7]$. With the advent of synthetic polymers, a second generation of biomaterials attracted enormous interest. New implant materials were developed with an emphasis to avoid a tissue response [8-10]. However, living tissues respond to e.g. changing physiological loads or biochemical stimuli, which led to numerous failures shortly after surgery or in the long term. At the end of the $20^{\text {th }}$ century it was recognized that the development of biomaterials should be centered around biomaterial-tissue interactions, which eventually led to current research on biologically based methods for the repair and regeneration of tissues. A third generation of biomaterials nowadays surfaced, which can stimulate specific cellular responses at the molecular level, thereby directing cell proliferation, differentiation and extracellular matrix deposition [11-15].

Here we describe a few design concepts, mainly focusing on in-situ forming hydrogels for biomedical and pharmaceutical applications that are shaping our future directions in the macromolecular engineering of biomaterials. In particular, we discuss materials based on the water soluble naturally occurring polymer dextran and the synthetic star shaped 8 arm poly(ethylene glycol).

\subsection{Dextran}


Dextran is a complex, branched and hydrophilic polysaccharide composed of anhydroglucose rings. Due to its hydrophilicity, biocompatibility and proven clinical applications this polysaccharide receives increasing interest in the (bio)medical and pharmaceutical fields. Because of the many hydroxyl functional groups present, dextran is an interesting polysaccharide as a starting biopolymer for the design of variety of structural motifs. It may be regarded as a natural alternative to the synthetic poly(ethylene glycol)s, which are studied intensively as a component of amphiphilic materials and in conjugation. It is therefore not surprising that chemical modification of dextran is increasingly studied for the design of novel materials with controlled properties.

This review highlights recent progress in the synthesis of new materials based on dextran. We summarize recent developments in the preparation of dextran derivatives with a focus on the chemical structure which make these materials e.g. applicable as prodrugs, bioactives, hydrogels or nanogels, nanoparticles and self-assembled structures for surface modification. Novel materials with properties intended for specific applications are for example nanoparticle formulations in the field of drug delivery systems and 3D scaffold materials useful in the tissue regeneration field. A number of reviews have been published on dextrans covering subjects like material production through biotechnological processes up to the chemical modification of this material. A recently published review paper of Heinze et al. [16] provides detailed information on structure and modification of dextran and is an excellent basis for researchers active in dextran research. Other review papers providing solid background on dextrans are the expert opinion paper of Sun on dextran conjugates in drug delivery [17], an overview of the typical industrial applications of dextran [18] and reviews in the field of hydrogels to be applied as drug delivery systems and tissue regeneration constructs [19-22].

\subsubsection{Structure and properties}

Dextrans are a family of neutral polysaccharides consisting of mainly $\alpha-(1 \rightarrow 6)$ linked D-glucose units in the main chain. These $\alpha-(1 \rightarrow 6)$ linkages in dextrans may vary from 50 to $97 \%$ of the total glycosidic bonds. Depending on the production process, branches comprising $\alpha-(1 \rightarrow 2), \alpha-(1 \rightarrow 3)$ and $\alpha-(1 \rightarrow 4)$ linkages may be present and molecular weights may vary from 5 to as high as $500 \mathrm{kDa}$ (Figure 2.1). 
Dextrans are industrially produced from sucrose by microorganisms that secrete dextran sucrases and a single enzyme can catalyze the synthesis of several types of linkages leading to different degrees of branching. The most commonly applied bacterial strains are Leuconostic mesenteroides NRRL B512F and Leuconostic mesenteroides NRRL B1299. The former gives dextran with 95\% $\alpha-(1-6)$ linkages and $5 \% \alpha-(1-3)$ linkages whereas the latter provides dextran with $63 \% \alpha-(1-6), 27 \% \alpha-(1-2)$ and $8 \% \alpha-(1-3)$ linkages. Depending on the microorganisms used the physical and chemical properties of dextrans produced can vary widely.

The production of dextrans remains an industrial challenge because of their wide use in many types of applications [23]. It is therefore still subject to research in order to provide a commercial attractive production process with control over the (branched) structure. As an example Sarwat et al. [24] recently showed that L. Mesenteroides CMG713 produces high molecular weight dextran without the presence of any branches.

Besides their application in the medical field dextrans are applied in the food industry, photographic industry and in cosmetics. Typical applications are their use in bakery products because of its moisturizing properties, ice cream, freeze dried foods, photographic silver emulsions and hair and skin care products [18].

The increasing interests in the use of dextrans in the biomedical field relates to its good biocompatibility, non-toxicity, hydrophilicity and degradation by dextranases which are present in the intestine, kidneys, lungs and spleen. These materials therefore have a long history to be applied in the medical and pharmaceutical fields. In the medical field dextrans have found applications as blood plasma extenders, blood flow improver, ophthalmic solutions and to preserve human tissues in transplantation. Especially, sulphated dextran can be used as a substitute for heparin in anticoagulant therapy. Dextrans also can be used to coat materials like quantum dots, and iron or gadolinium nano spheres in medical imaging.

Aqueous solutions of dextrans having molecular weights $(\mathrm{Mw})$ of 40.000 and 70.000 are commercially available as clinical grade dextrans. These materials have a high heat resistance in sterilization and good storage capability. Dextran with molecular weight of 40.000 improves blood flow and inhibits erythrocyte aggregation. 
Hypersensitivity reactions can be limited by a pre-injection of low molecular weight dextran (Mw $1000 \mathrm{Da})$ as a monovalent hapten [23,24].

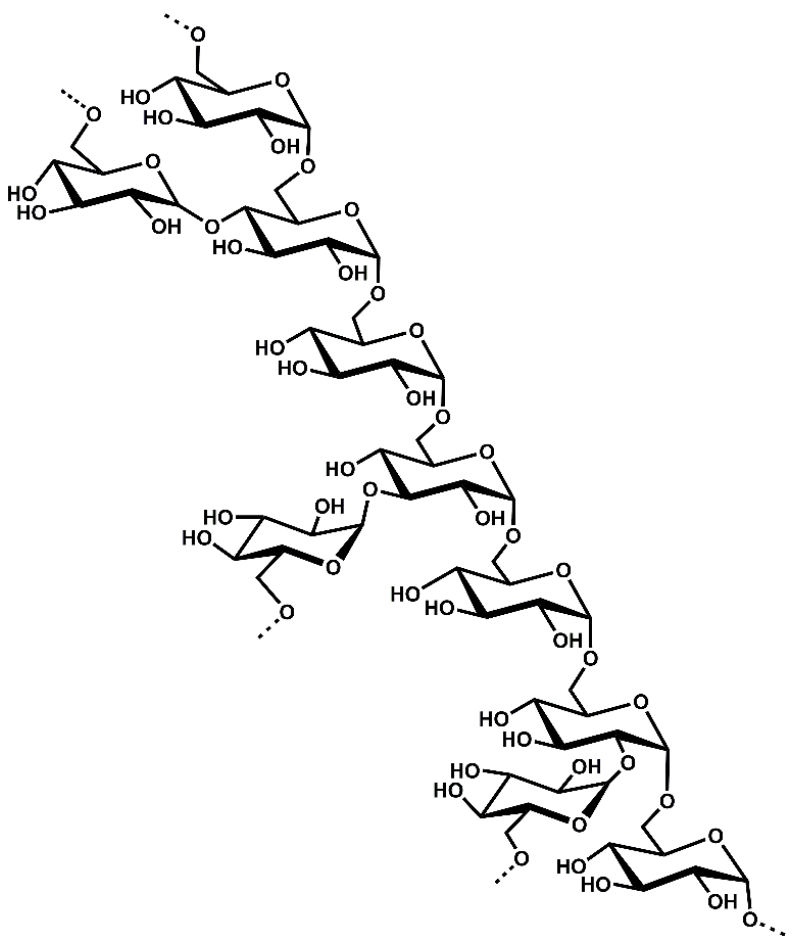

Figure 2.1 Dextran is a polysaccharide of $\alpha-1,6$ linked glucose units with branches at the 2, 3 and or 4 positions.

Dextran only has hydroxyl functional groups, different from many other polysaccharides like chitosan, alginate and hyaluronic acid. The structure of dextrans has been investigated in detail [16]. The analysis of the degree of branching and length of branches is complicated and not always feasible using NMR techniques. Derivatization by methylation of dextran hydroxyl groups, partly oxidation of rings and degradation have been employed to gain a more detailed structural analysis. Dextrans are very well soluble in water and polar solvents like dimethyl sulfoxide, formamide, and ethylene glycol. In solution most dextran molecules behave as flexible polymeric chains and behave as a random coil. As the temperature is increased from $20{ }^{\circ} \mathrm{C}$ to 40 ${ }^{\circ} \mathrm{C}$, dextran is driven toward theta conditions in water. Upon heating, the polymer 
segments interact less with the solvent molecules, adopt a less expanded conformation in solution, and the intermolecular association with other polymer segments is enhanced. The decrease in the hydrogen bonding interactions appears to be the reason for dextran coils to contract. The radius of gyration follows Rcoil (nm) Mw1/2 [25]. The lower molecular weight dextrans used in clinical applications can be obtained from high molecular weight dextrans by acid hydrolysis. On the other hand controlling the sucrose concentration in the biotechnological process may afford controlled molecular weights of dextran.

The presence of only hydroxyl groups in dextran allows the introduction of other functional groups randomly distributed on the polymer chain. Depending on the degree of substitution, and physical properties of the groups introduced, the properties of dextrans like inherent hydrophilicity are changed and even become insoluble in water or physiological media. The following sections refer to recent developments in dextran conjugates.

\subsubsection{Dextran conjugates}

In recent years several research groups have focused on the synthesis of dextran conjugates to make new materials that may be applied in the biomedical and pharmaceutical fields. The examples given below provide trends observed, but this overview will not be exhaustive. In Figure 2.2 examples are presented of the conversion of dextran into dextran esters for the preparation of novel materials.

\subsubsection{Dextran esters}

$\mathrm{N}, \mathrm{N}$ '-carbonyl-diimidazole (CDI) activation of carboxylic acid groups is known as an efficient method for the preparation of esters [16]. As an example, using in-situ activation, ibuprofen and naproxen dextran conjugates with high degree of substitution were prepared. These conjugates are soluble in organic solvents and nanoparticles with consequently high loading of the drug were prepared by precipitation in dilute aqueous solutions. Nano particle formulations with a uniform distribution can be prepared in this way without the addition of a surfactant. The dextran esters with high degree of 
substitution are highly stable to hydrolysis in a wide $\mathrm{pH}$ range. At low degree of substitution, the release of loaded drugs may take place, but possibly also relies on the enzymatic cleavage of the dextran by dextranase [26]. The group of Heinze also prepared aliphatic dextran esters with adjustable degree of substitution (DS), which appear well suitable to be applied as coating materials. Such materials are biocompatible for they do not influence cell growth. Preliminary experiments have shown that dextran esters with long aliphatic chains can also act as an adhesive [27].

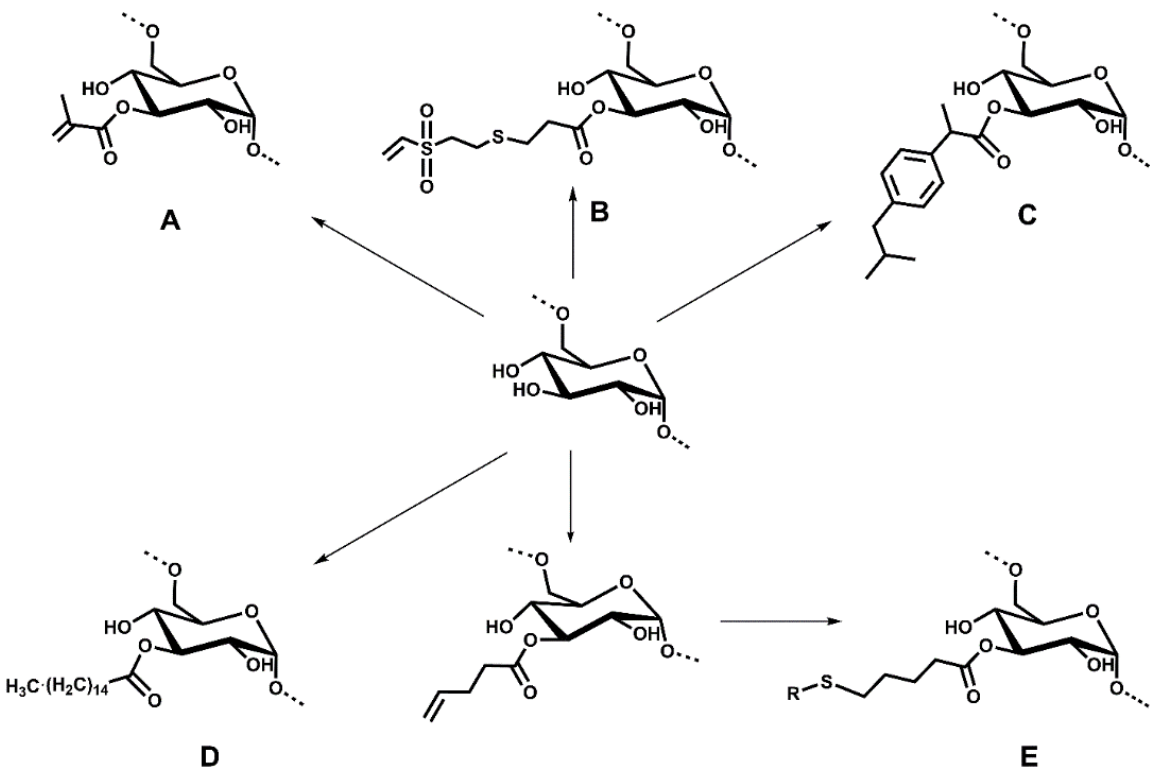

Figure 2.2 Schematic overview of dextran esters from literatures. A; acrylate [22], B; vinyl sulfonic ester [32], C; drug conjugate [26], D; aliphatic ester conjugate [27], E; thiolated conjugate [30].

In-situ CDI activation has been employed to introduce furoyl-, pyroglutamyl-, propyl-, and acetyl moieties onto dextran with varying degrees of substitution. A DS of at least 2 of the hydroxyl groups present in each ring was necessary to prepare nanoparticle formulations. Such materials may be used for the release of hydrophobic drugs [28]. In contrast to a chemically catalyzed reaction an enzyme mediated transesterification using vinyl dodecanoate and lipase as a catalyst has shown an effective way to introduce ester groups to the dextran backbone. The reaction conducted 
in dimethyl sulfoxide affords after fractionation a range of amphiphilic materials that may be used in the preparation of biodegradable micro or nano particles [29].

Acid chlorides or anhydrides can be applied for preparing dextrans with conjugate ester groups. These esters may contain other functional groups that can be used in further modification. As an example conjugate esters comprising an isolated double bond were subjected to subsequent thiol-ene chemistry. This reaction is regarded as a click reaction, which leads to the formation of thiol-ether linkage. This method does not rely on the use of copper ions like the copper-mediated 1,3-dipolar cycloaddition of an alkyne and azide, which has become most popular in click chemistry [30]. The reaction was performed under physiological conditions and is initiated thermally or by photoinitiation. Mergy et al. synthesized an alkene-functional high molecular weight dextran using the corresponding anhydride and showed that a variety of (end) functionalized thiols can be coupled to the double bonds present. The number of groups conjugated can be controlled (10\% to 55\% of substituted rings). The thiol-ene coupling reaction is induced using UV light in the presence of the water-soluble photoinitiator, Irgacure 2959. A quantitative reaction is generally observed within seconds at ambient temperatures. Interestingly, in the hydrogel formation of the conjugated dextran with a linear PEG dithiol, the increase of the storage modulus G' with concentration was much higher above a critical concentration of $25 \mathrm{~g} / \mathrm{L}$. Above this concentration, which is close to the overlap concentration of dextran ( $\mathrm{Mw}$ of the dextran was $200.000 \mathrm{~g} / \mathrm{mol}$ ), interpolymer cross-linking plays an important role, whereas intramolecular crosslinking is the dominant process at low concentrations.

Dextran conjugates carrying vinyl sulfone groups have been designed for the preparation of in-situ forming hydrogels through Michael type addition reaction. Vinyl sulfone alkonoic acids were prepared and coupled to dextran using using N,N'dicyclohexylcarbodiimide (DCC) and 4-(dimethylamino)pyridinium-4-toluenesulfonate (DPTS) using DMSO as a solvent. The degree of substitution was varied and hydrogel formation was accomplished by reaction with a four armed PEG bearing thiol end-groups. These hydrogels are degraded through hydrolysis of the ester bonds. The degradation time depends on the concentration, degree of substitution and also the length of the spacer between the thiol-ether and ester group. The latter is due to the decreased positive charge on the carbonyl carbon making it less susceptible to 
hydrolysis [31]. Such type of materials recently revealed good biocompatibility, fast gelation and good mechanical properties [32].

\subsubsection{Dextran carbonates}

Dextran alkyl carbonates with high DS can be conveniently prepared by reaction of dextran hydroxyl groups with different acylation agents. The use of CDI allows the manufacturing of dextran carbonates based on tertiary alcohols or complex molecules of pharmacological interest. The conjugation reaction appeared highly efficient and was shown a general methodology to produce dextran carbonates. The structure of several dextran carbonates were presented in detail and show that groups are randomly distributed on the dextran backbone [33]. The formation of dextran carbonates from fluoroformates as acylating agents has also been shown highly efficient. The formation of nano particles from conjugates with a high degree of substitution was shown and such particles may potentially be applied for delivery of therapeutic or imaging agents [34]. In a similar way Chiang et al prepared activated octadecanol by reaction with CDI. Conjugation to dextran afforded materials that can be assembled into polymersomes with controlled dimensions [35]. The group of Fréchet has developed a protocol to prepare $\mathrm{pH}$-sensitive dextrans conjugated with azide groups through a hydrophilic linker. The remaining hydroxyl groups were protected by acetal moieties. This latter modification makes the dextran soluble in organic solvents and enables via a double emulsion process the preparation of particles. The reactive azide functional groups mainly located on the surface of the particles were accessible for further modification as shown by a click reaction using an alkynylated mannoside [36].

\subsubsection{Dextran carbamates}

As shown above the synthesis of dextran conjugates generally relies on reactions of preactivated molecules. Similarly, a versatile method to prepare dextran conjugates is the activation of the dextran alcohol groups with p-nitrophenyl chloroformate to give carbonate groups. The method has been described in detail [37] and subsequent reaction with amines allows conjugation with the formation of a hydrolytically stable carbamate 
bond. This method of conjugation has been used to couple phenolic groups to the dextran backbone which were subsequently subjected to enzymatic crosslinking using Horse radish peroxidase as an enzyme in the presence of hydrogen peroxide (Figure 3.5.3). The enzyme generates radicals on the phenolic groups which by combination form the crosslinks. Depending on the degree of substitution of tyramine groups, polymer concentration and polymer molecular weight the gelation time can be adjusted.

It has been shown that low enzyme concentrations and importantly low hydrogen peroxide concentrations can be used making these type of hydrogels biocompatible. Such type of hydrogels can be formed in-situ making them applicable as injectable hydrogels. This method can also be applied to tyramine conjugates of e.g. hyaluronic acid and heparin and co-crosslinking of different polysaccharide conjugates provides hydrogels that may be applied in the various fields of tissue regeneration or drug delivery systems [38-40].

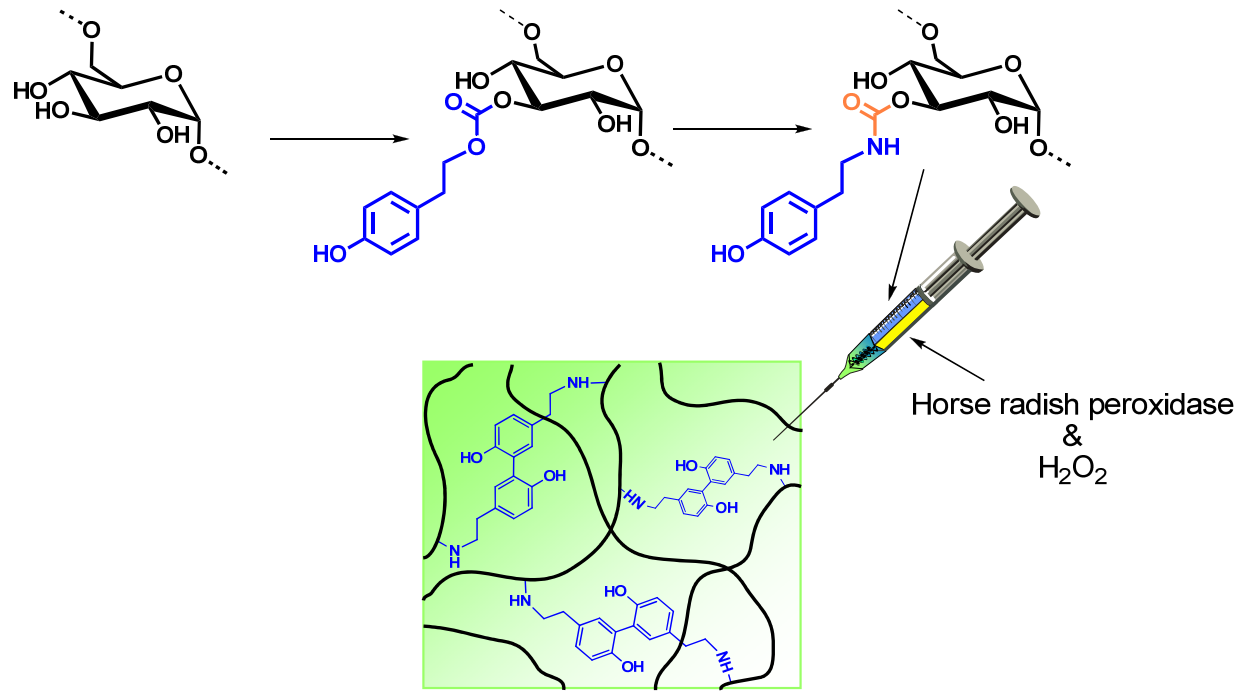

Figure 2.3 Schematic representation of an injectable dextran tyramine conjugate hydrogel [38].

Another method to prepare dextran carbamates is the reaction of the alcohol groups with isocyanates. The group of Yang used the commercial available 2-isocyanatoethyl methacrylate to prepare dextran conjugated with acrylate groups and studied the 
photocrosslinking. The conjugates with lower degree of substitution are well soluble in water. The adhesion strength was measured by placing the material between gelatin coated glass slides and adhesive strengths were in the order of a few MPa. The adhesion strength could be further improved by the post oxidation of the grafted dextran. The introduced aldehyde groups can form Schiff bases with the $\mathcal{E}$-amino groups present in the gelatin. In this way a dually crosslinked network is formed [41,42].

\subsubsection{Dextran copolymers}

\subsubsection{Graft copolymers}

Van Tomme and Hennink reviewed the large variety of dextran conjugates that have been used in the preparation of systems for the controlled release of proteins [22]. Part of their work has been directed to the conjugation of short poly(lactide) chains to dextran and the formation of stereocomplexes from enantiomeric graft copolymers. Coupling of poly(lactide) chains was performed by CDI activation of oligolactates. In the next step, L- and D-lactic acid oligomers were coupled via their terminal hydroxyl group to dextran. Upon mixing aqueous solutions, a hydrogel is formed through the formation of stereocomplexes that act as crosslinks. These hydrogels show thermoreversibility due to the reversibility of the crosslinks formed. The storage modulus of the obtained hydrogel decreased upon heating to $80{ }^{\circ} \mathrm{C}$, while it was restored upon cooling to $20^{\circ} \mathrm{C}$. Interestingly, gel formation was favored when lactic oligomers were coupled via their hydroxyl groups, whereas the oligomers of opposite chirality were coupled via their carboxylic acid groups. This is ascribed to the parallel packing of the oligomers in stereocomplexes.

Dextran grafted with poly(lactide) chains have been developed for nanoparticle formation in the controlled release of drugs and as surfactants [43]. The molecular weight of the polyester chains can be varied as well as the degree of substitution of the grafts to the dextran backbone. It was shown that high degrees of substitution renders the dextran backbone slower to degrade by dextranases.

Coupling the polyester poly(3-hydroxyalkanoates) to dextran has also recently been performed using click chemistry [44]. Dextran was modified with azide groups, a 
methodology also described by the group of Heinze [45] and reacted with alkyne terminated polyester oligomers. These materials were studied for their nanoparticle formation with the intention to be applied as vehicles for the release of hydrophobic drugs. Acrylated dextrans have been extensively studied for the preparation of hydrogels to applied in the biomedical and pharmaceutical field [22]. Grafting of a variety of (meth)acrylate groups has been accomplished. The broad scope of materials that can be prepared offers the opportunity to make smart materials that are sensitive to $\mathrm{pH}$, temperature or light. A few examples may illustrate this. Klinger and Lanfester introduced a photo-cleavable linker in between dextran and the acrylate group, thereby giving the possibility to disintegrate networks [46]. Dextran conjugated with hydroxyethyl methacrylate and subsequently polymerized in the presence of methacrylic acid or dimethylaminoethyl methacrylate, negatively and positively charged materials are obtained. Mixing these materials gives hydrogels bonding together by ionic interactions.

Sun and Mao recently reviewed their work on cationically and anionically modified dextrans and studied the assembly process [17]. Nano and macro structures like spheres tubules and 3D networks have been prepared. Such structures can be applied as drug delivery systems or may function as scaffolds in tissue engineering applications or temperature sensitive cell detaching substrates.

\subsubsection{Block copolymers}

Dextrans can also be coupled to synthetic polymers through the polysaccharide reducing end. Up to now only a limited number of researchers have focused on the preparation and properties of dextran-based block copolymers (Figure 3.5.4). Methods comprise the introduction of an amino group or carboxylic acid group at the reducing end by chemical modification and subsequent coupling a complementary end-functionalized polymer. Dextran-polystyrene diblock copolymers $[47,48]$ and biodegradable dextranpoly( $\varepsilon$-caprolactone) diblock copolymers $[49,50]$ have been synthesized by such type of coupling reactions. Click chemistry has been used to prepare a dextran-poly $(\gamma$-benzyl 1glutamate) diblock copolymer [51]. The aqueous self-assembly into micelles, vesicles and polymersomes has been studied in relation to the potential application as drug 
delivery systems. Sun et al. reported a reduction sensitive dextran-SS-poly( $(\varepsilon-$ caprolactone) diblock copolymer which may be applied in the intracellular delivery of cytostatic agents [52].

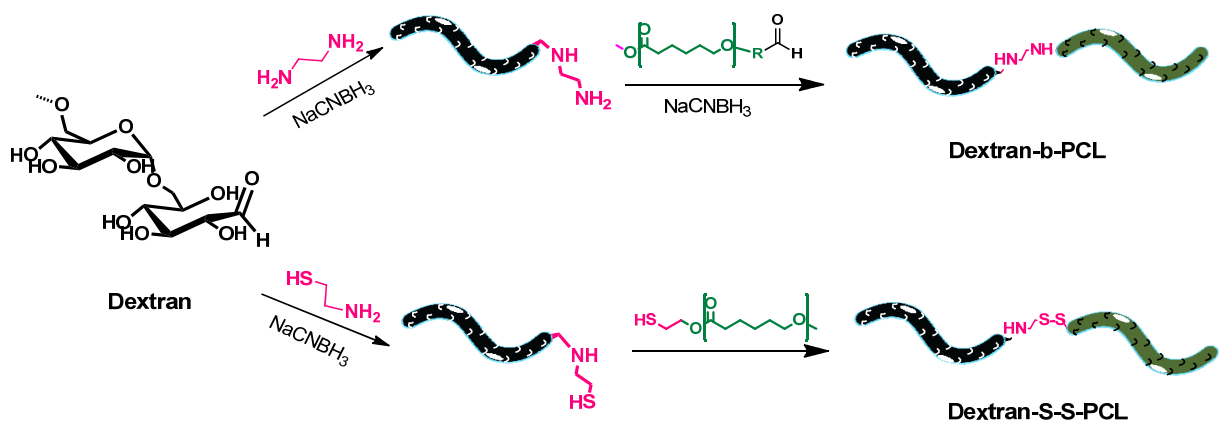

Figure 2.4 Examples of dextran poly( $\varepsilon$-caprolactone) block copolymers $[50,52]$.

In a recent paper, Novoa-Carball and Müller showed that dextran can be coupled to PEG by an oxime click reaction from a readily available amino end-functionalized PEG [53]. The method is highly efficient and may be used for other polysaccharides carrying a reductive end unit. Such block copolymers can be post modified into polyanions by carboxymethylation of the dextran block [54]. The above given examples show that converting the reductive end moiety in dextran into a reactive functional group allows for the design and preparation of many structural motifs. As an example this methodology has been used to couple an amine end-modified dextran to carboxylic acid groups of hyaluronic acid thus obtaining a branched polysaccharide structure [40].

Dextrans can also be coupled to synthetic polymers through the polysaccharide reducing end. Up to now only a limited number of researchers have focused on the preparation and properties of dextran-based block copolymers (Figure 3.5.4). Methods comprise the introduction of an amino group or carboxylic acid group at the reducing end by chemical modification and subsequent coupling a complementary endfunctionalized polymer. Dextran-polystyrene diblock copolymers [47,48] and biodegradable dextran-poly( $\varepsilon$-caprolactone) diblock copolymers [49,50] have been synthesized by such type of coupling reactions. Click chemistry has been used to prepare a dextran-poly( $\gamma$-benzyl l-glutamate) diblock copolymer [51]. The aqueous self- 
assembly into micelles, vesicles and polymersomes has been studied in relation to the potential application as drug delivery systems. Sun et al. reported a reduction sensitive

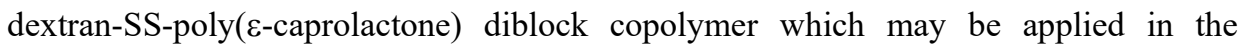
intracellular delivery of cytostatic agents [52].

In a recent paper, Novoa-Carball and Müller showed that dextran can be coupled to PEG by an oxime click reaction from a readily available amino end-functionalized PEG [53]. The method is highly efficient and may be used for other polysaccharides carrying a reductive end unit. Such block copolymers can be post modified into polyanions by carboxymethylation of the dextran block [54]. The above given examples show that converting the reductive end moiety in dextran into a reactive functional group allows for the design and preparation of many structural motifs. As an example this methodology has been used to couple an amine end-modified dextran to carboxylic acid groups of hyaluronic acid thus obtaining a branched polysaccharide structure [40].

\subsubsection{Degradation}

Dextran is degraded enzymatically by dextranase, which is present in the human body in the colon, spleen, kidneys and lungs. This enzymatic degradation can be regarded important in the application of dextran and dextran conjugates. High degrees of substitution renders the dextran non degradable by the enzyme and degradation may rely on the detachment of conjugated groups [55]. An assay for the degradation of dextran by dextranase can be found in papers of the group of Durand [43]. In another paper the enzymatic degradation of conjugated dextrans in the presence of dextranase was compared to that of native dextran. For dextran with a molecular weight of 40.000, it was shown that the main degradation product was isomaltose. It was also shown that the average number of glucose units per degraded dextran chains increases significantly with higher degrees of substitution of conjugated groups. It was found that among all the dextran conjugates tested, only dextrans with a substitution ratio lower than 100 could be partially degraded in the presence of dextranase [55].

Ester groups conjugated to dextran may be hydrolyzed in a physiological environment if there is not a high degree of steric hindrance and if e.g. the charge density on the carbonyl carbon is not delocalized to a high degree. Conjugated 
molecules linked by ester groups or e.g. grafted poly(lactide) chains can be detached from the polymer backbone by preferential hydrolysis of the ester linkage or carbonate linkage $[22,56]$.

\subsubsection{Outlook}

The use of dextran conjugates receives increasing interest in the development of new materials for biomedical and pharmaceutical applications. Dextran conjugates have become readily available nowadays and such materials may be used in polymer assemblies like nanoparticles using a proper balance between hydrophobic conjugated groups and the hydrophilic dextran. The high functionality of the dextran backbone provides the preparation of architectures like block, graft and comb type materials. Such materials may be applied in tissue engineering as scaffolds, like hydrogels and as coating materials. The already proven applicability of dextrans in the clinic provides an excellent starting point for future research in this field.

\subsection{Poly(ethylene glycol)}

Poly(ethylene glycol)s (PEGs) are hydrophilic synthetic polymers and have been used in various biomedical applications. Due to an organized hydration shell, PEGs do not have specific interactions with biological components and cells (no immune response) [57]. Therefore, it is a suitable material to modify therapeutic proteins and peptides to increase their solubility, lower their toxicity as well as prolong their in vivo circulation time [58-63]. Hydrogels based on synthetic materials like PEG show the characteristics of a 3D interstitial crosslinked network that swells in aqueous environments with a water content similar to soft tissues, which make them highly biocompatible. These PEG hydrogels are generally chemically well-defined, and multiple chemistries can be used for functional modifications. Specifically, branched PEGs like the star shaped 8 arm PEG have been extensively studied in the preparation of hydrogels for tissue engineering and drug delivery applications. Because of the multiple reactive end-groups present 8 PEG macromeres can be easily converted into hydrogels. An overview of the 
chemistries involved for preparation of 8 arm PEG based hydrogels was summarized in this chapter.

\subsubsection{Hydrogels by chemically cross-linked 8PEG-derivatives}

The use of an 8 arm PEG for the preparation of chemically crosslinked hydrogels was approached using different strategies. The general requirements with respect to in-situ forming hydrogels are the coupling of functional groups without the release of potentially toxic molecules and short gelation times.

In early work of the group of Hubbell, an 8 arm PEG extended with oligo(DL-lactic acid) or oligo(glycolic acid) blocks and terminated with acrylate groups were synthesized. Photopolymerization was applied for hydrogel formation in the presence of the photoinitiator Irgacure 2959. The hydrogels degraded through hydrolysis of the oligo( $\alpha$-hydroxyl acid) blocks depending on the composition, in between 1 day and 4 months [64].

Buwalda et al. prepared hydrogels by using 8 arm PEG-PLLA-acrylates and 8 arm PEG-thiols through a Michael-type addition. Hydrogels were formed within 1 min and quickly reached a high storage modulus up to $17 \mathrm{kPa}$. Such a hydrogel was used to release lysozyme from the hydrogel. Release was regulated by diffusion and degradation while the protein activity was maintained [65]. In a recent study, Zhang and co-workers showed that an 8 arm PEG based hydrogel could be prepared by an amineMichael type addition reaction. An 8 arm PEG with acrylate end groups was crosslinked using a $30 \%$ ammonia solution, and depending on the concentration the gelation time was modulated from $168 \mathrm{~min}$ to $5 \mathrm{~min}$. Moreover, residual acrylate groups could be bio-functionalized via a second Michael-type addition using thiol-containing bioactive molecules or photocrosslinked to give hydrogels with a higher mechanical integrity [66].

Mixing dextran aldehyde and an 8 arm PEG amine afforded an adhesive hydrogel crosslinked by Schiff-base linkages. In vitro cytotoxicity experiments revealed that the hydrogels are biocompatible and in vivo experiments on sealing and healing of corneal incisions of rabbit eyes indicated that hydrogels maintained an eye leak pressure of at least $120 \mathrm{~mm} \mathrm{Hg}$ and remained visibly present at the wound site for 5 days [67]. 
Similarly, Fan and co-workers reported the formation of hydrogels by Schiff-base reaction between 4 or 8 arm PEG amines and aldehyde groups of oxidized hyaluronic acid [70].
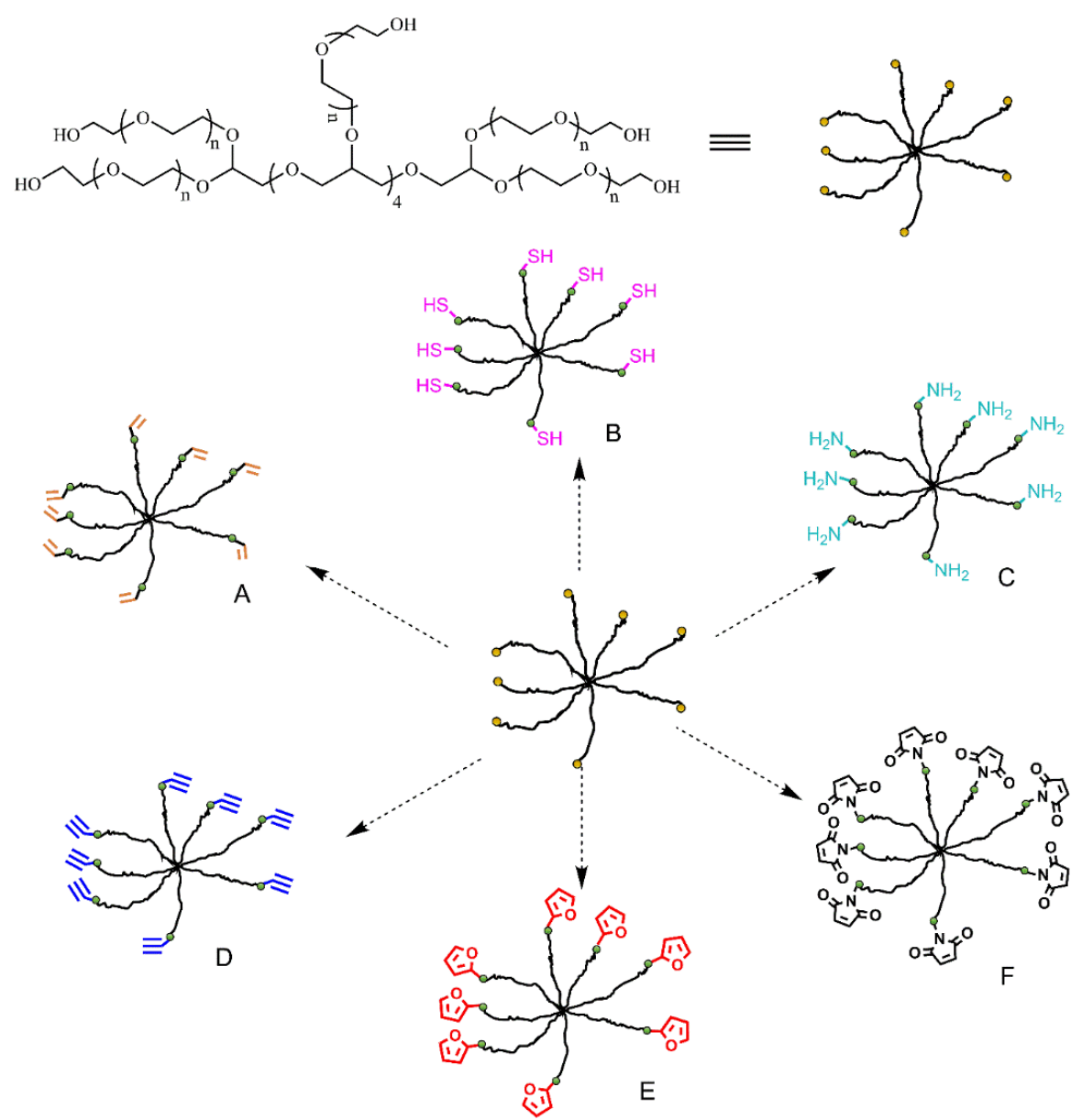

Figure 2.5 Schematic overview of chemically cross-linked 8 arm PEG end functionalized with different groups. A: acrylate [64], B: thiolate [65], C: amine [67], D: alkyne [68], E: furyl and F: maleimide [69].

The group of Anseth reported a stimulus-responsive hydrogel made from an 8 arm PEG with alkyne end groups and an azide-fuctionalized photodegradable cosslinker. Hydrogels showed bulk degradation upon exposure to light which was dependent on exposure time, wavelength and light intensity. The changes in local microenvironments 
induced by controlled degradation of the scaffold matrix may change cell-matrix interactions. Such type of hydrogels may therefore provide fundamental insight in tissue regeneration processes [68].

Crosslinking 8 arm PEG macromonomers end functionalized with furyl and maleimide groups takes place via a Diels-Alder reaction. The cross-linking and degradation were dependent on the $\mathrm{pH}$ and temperature. Low $\mathrm{pH}(\mathrm{pH} 3.0$ vs $\mathrm{pH} 7.4)$ showed faster gelation times ( $88 \mathrm{~min}$ vs $375 \mathrm{~min}$ ). At higher temperatures $\left(50^{\circ} \mathrm{C}\right.$ vs 20 ${ }^{\circ} \mathrm{C}$ ) faster degradation ( 2 days vs 98 days) was observed. A retro-Diels-Alder reaction followed by a base-catalyzed ring-opening hydrolysis of maleimide groups to unreactive maleamic acid derivatives was accounted for the degradation [69].

\subsubsection{Hydrogels by physically cross-linking of 8PEG derivatives}

Because physical crosslinking is based on non-covalent interactions only mild crosslinking conditions and no additives are needed. These mild conditions may be used to entrap labile compounds, like proteins, drugs or bioactive molecules. The properties of physically cross-linked gels may vary widely, especially when the interactions are governed by dynamic equilibrium processes. On the other hand, in the design of intelligent hydrogels that respond to various external stimuli are easily prepared by physical crosslinking.

L- or D-Lactide ring-opening polymerization using the end hydroxyl groups of an 8 arm PEG as initiator provides enantiomeric star block copolymers upon mixing aqueous solutions of these polymers afford hydrogels by stereocomplexation. Stereocomplexed hydrogels were stable up to $70{ }^{\circ} \mathrm{C}$ and had high storage moduli up to $14 \mathrm{kPa}$. Protein (lysozyme) release from these hydrogels followed first order kinetics and about $90 \%$ was released in 10 days. Larger proteins like IgG and the therapeutic protein rhIL-2 followed a zero order release kinetics. Approximately $50 \%$ and $45 \%$ of these proteins was released in 16 and 7 days, respectively [71,72]. The group of Ohya and co-workers designed irreversible temperature-responsive high strength hydrogels from an enantiomeric mixture of starbursts triblock copolymers of 8 arm PEG and PLLA or PDLA, these hydrogels were completely biodegraded in about 20 days, demonstrating a potential use as injectable biomaterials for drug delivery [73]. Furthermore, this group 
synthesized partially cholesterol-substituted 8 arm PEG-b-PLLA. Hydrogels were formed by increasing the temperature above $34{ }^{\circ} \mathrm{C}$ at polymer concentrations above 3 wt $\%$. L929 cells encapsulated in the hydrogels were viable and proliferated in the threedimensional structure. At physiological conditions, hydrogels were completely dissociated in about 1 month [74].
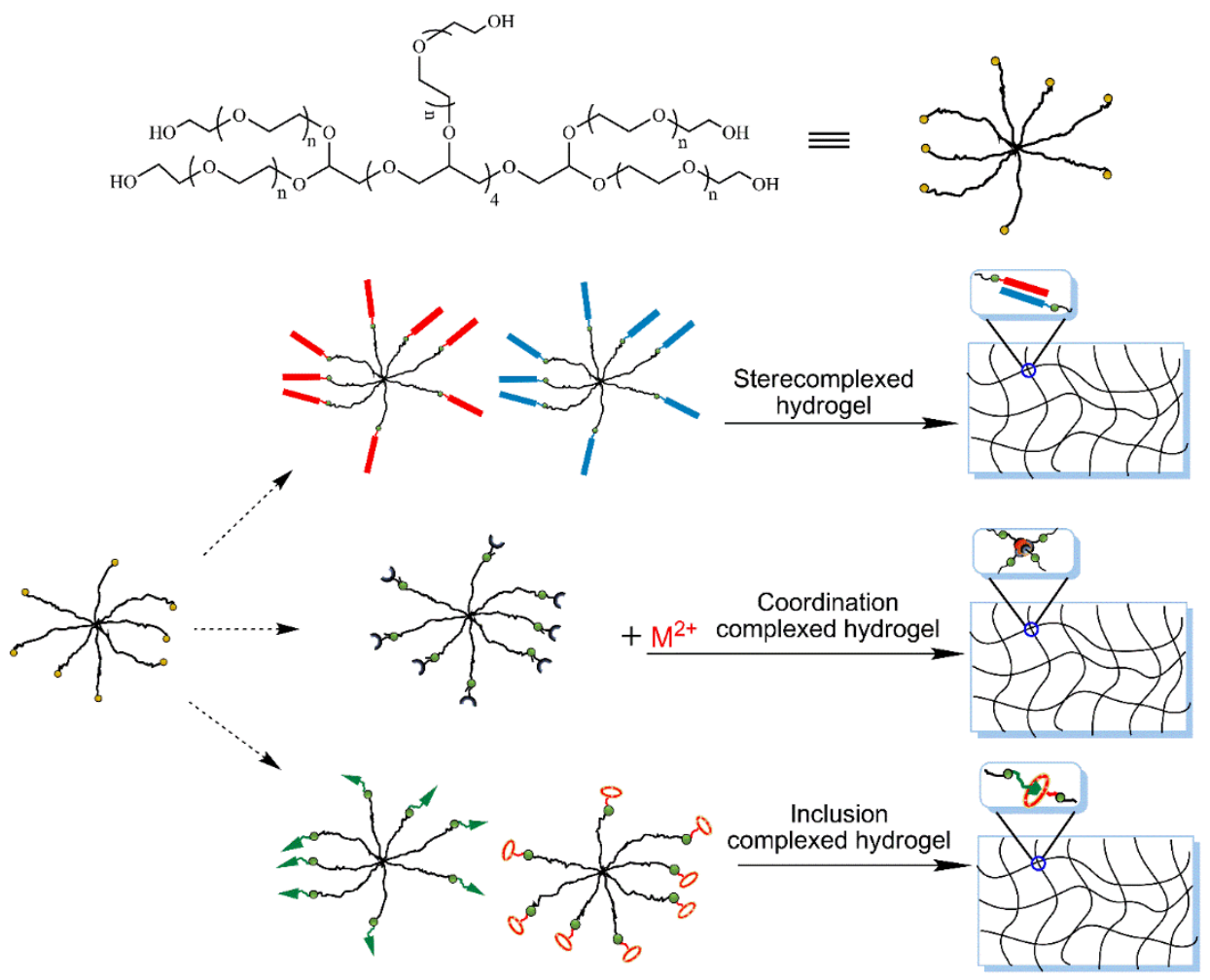

Figure 2.6 Schematic overview of physically cross-linked hydrogels from 8 arm PEG end functionalized with different groups. A: stereocomplexed hydrogel of 8PEG-PLLA and 8PEG-PDLA [71], B: coordination complexed hydrogel of 8PEG-PLLA-pyridine with metal ions [75] and $\mathrm{C}$ : inclusion complexed hydrogel of 8PEG-cholesterol with 8PEG- $\beta$-cyclodextrin [76].

The group of Hennink reported a novel self-assembling 8 arm PEG hydrogel system based on inclusion complexes of $\beta$-cyclodextrin $(\beta C D)$ and cholesterol-derived 8 arm PEG in aqueous solutions. Rheological studies revealed that hydrogels are fully 
thermoreversible. Furthermore, the in vitro degradation mechanism and protein release behavior were evaluated. It was found that depending on the hydrogel composition and the initial crosslink density, the degradation mainly takes place by surface erosion, leading to a quantitative and nearly zero-order release of entrapped proteins [76,77].

Metallo supramolecular crosslinking of a pyridine end functionalized 8 arm PEGPLLA block copolymer was investigated in the group of Feijen. In situ forming hydrogels by metallo supramolecular assemblies with transition metal ions $\left(\mathrm{Cu}^{2+}, \mathrm{Co}^{2+}\right.$ and $\mathrm{Mn}^{2+}$ ) were prepared. These complexes showed a shift in thermoreversible gelation to higher temperatures [75].

\subsubsection{Dually cross-linked hydrogels from 8 PEG derivatives}

The large number of studies on physically and chemically cross-linked hydrogels opened possibilities to combine both crosslinking methods in their macromolecular engineering. Although physically crosslinked hydrogels are mechanically weak, the kinetic gel formation may be fast. Post formation of chemical crosslinking in the hydrogel 3D network will strengthen the constructs.

Hiemstra et. al reported a new approach to prepare in situ forming biodegradable robust hydrogels based on 8 arm PEG derivatives by combining stereocomplexation and photopolymerization. Two types of methacrylate-functionalized 8PEG(-MA)-PLLA(MA) and 8PEG(-MA)-PDLA(-MA) star block copolymers were synthesized. Results showed that methacrylate groups had no influence on stereocomplexation, whereas additional photopolymerization significantly increased the storage moduli as compared to hydrogels formed by sterocomplexation only. In particular, photopolymerization of stereocomplexed hydrogels was possible at very low initiator concentrations $(0.003$ wt $\%)$. Degradation experiments showed that the dually cross-linked networks degraded much slower than only photopolymerized or stereocomplexed crosslinked hydrogels. This strategy provides a novel approach to obtain fast in situ forming robust hydrogels. 

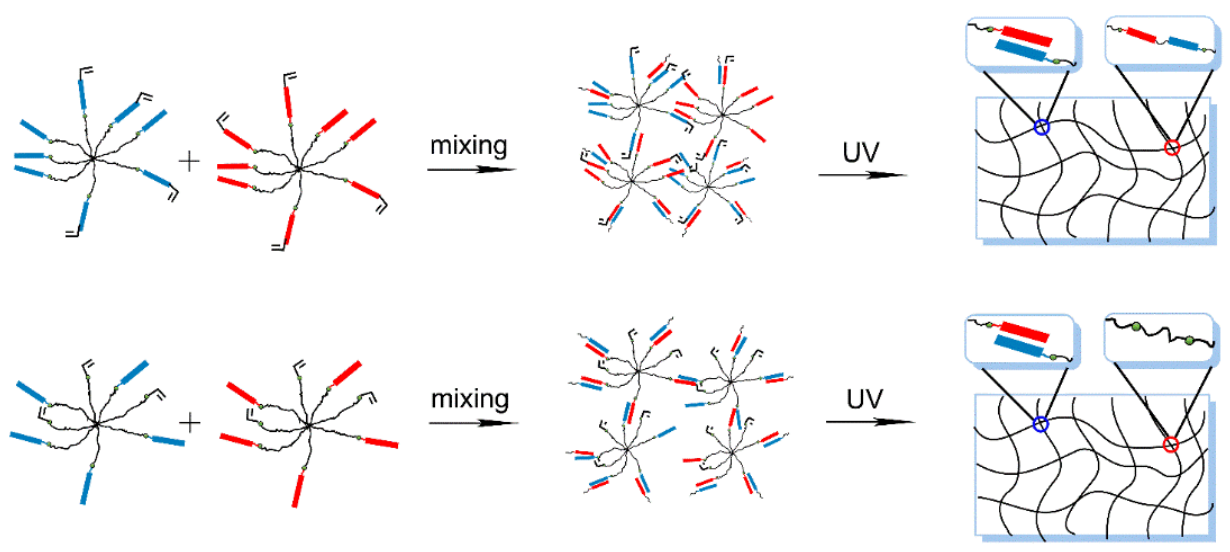

Figure 2.7 Schematic representation of the preparation of stereo and stereo-photo crosslinked hydrogels based on PEG-PLA-MA or PEG-MA/PLA star block copolymers [78].

\subsubsection{Outlook}

Controlled end-group modification of 8 arm PEG allows the preparation of variety of crosslinked hydrogels. Degradable linkages are easily introduced and allows PEG hydrogels to be degraded in the controlled way, creating possibilities for new ECM deposition, release of therapeutic agents or allows manipulation of the chemical and physical properties of hydrogels.

\section{Reference}

[1] Langer, R.; Tirrell, D. A., Designing materials for biology and medicine. Nature 2004, 428 (6982), 487-492.

[2] Hench, L., Biomaterials. Science 1980, 208 (4446), 826-831.

[3] Langer, R., Biomaterials in drug delivery and tissue engineering: One laboratory's experience. Acc. Chem. Res. 2000, 33 (2), 94-101.

[4] Hubbell, J. A., Biomaterials in tissue engineering. Bio-Technology 1995, 13 (6), $565-576$. 
[5] Ratner, B. D.; Bryant, S. J., Biomaterials: Where we have been and where we are going. Annu. Rev. Biomed. Eng. 2004, 6, 41-75.

[6] Peppas, N.; Langer, R., New challenges in biomaterials. Science 1994, 263 (5154), 1715-1720.

[7] Staiger, M. P.; Pietak, A. M.; Huadmai, J.; Dias, G., Magnesium and its alloys as orthopedic biomaterials: A review. Biomaterials 2006, 27 (9), 1728-1734.

[8] Nair, L. S.; Laurencin, C. T., Biodegradable polymers as biomaterials. Prog. Polym. Sci. 2007, 32 (89), 762-798.

[9] Griffith, L. G., Polymeric biomaterials. Acta Mater. 2000, 48 (1), 263-277.

[10] Seal, B. L.; Otero, T. C.; Panitch, A., Polymeric biomaterials for tissue and organ regeneration. Materials Science \& Engineering R-Reports 2001, 34 (45), 147-230.

[11] Francis Suh, J. K.; Matthew, H. W. T., Application of chitosan-based polysaccharide biomaterials in cartilage tissue engineering: a review. Biomaterials 2000, 21 (24), 2589-2598.

[12] Zhang, S., Fabrication of novel biomaterials through molecular self-assembly. Nat Biotech 2003, 21 (10), 1171-1178.

[13] Anderson, J. M.; Rodriguez, A.; Chang, D. T., Foreign body reaction to biomaterials. Semin. Immunol. 2008, 20 (2), 86-100.

[14] Lutolf, M. P.; Hubbell, J. A., Synthetic biomaterials as instructive extracellular microenvironments for morphogenesis in tissue engineering. Nat. Biotechnol. 2005, 23 (1), 47-55.

[15] Hench, L. L.; Polak, J. M., Third-Generation Biomedical Materials. Science 2002, 295 (5557), 1014-1017.

[16] Heinze, T.; Liebert, T.; Heublein, B.; Hornig, S. In Polysaccharides Ii; Klemm, D., Ed.; Springer-Verlag Berlin: Berlin, 2006; Vol. 205, p 199-291.

[17] Sun, G.; Mao, J. J., Engineering dextran-based scaffolds for drug delivery and tissue repair. Nanomedicine 2012, 7 (11), 1771-1784.

[18] A.Lakshmi Bhavani, J. N., Dextran - the polysacchride with versatile uses. Int J Pharma Bio Sci. 2010, 1 (4), 569-573.

[19] Censi, R.; Di Martino, P.; Vermonden, T.; Hennink, W. E., Hydrogels for protein delivery in tissue engineering. J. Controlled Release 2012, 161 (2), 680-692. 
[20] Hennink, W. E.; van Nostrum, C. F., Novel crosslinking methods to design hydrogels. Adv. Drug Del. Rev. 2012, 64, 223-236.

[21] Varshosaz, J., Dextran conjugates in drug delivery. Expert Opin. Drug Deliv. 2012, $9(5), 509-523$.

[22] Van Tomme, S. R.; Hennink, W. E., Biodegradable dextran hydrogels for protein delivery applications. Expert Rev. Med. Devices 2007, 4 (2), 147-164.

[23] Mary Helen P. B. Vettoria, K. C. B., Mariana Cortezia,; Cristian J. B. de Limab, J. C., Dextran: effect of process parameters on production, purification and molecular weight and recent applications. Diálogos \& Ciência 2012, 31, 171-186.

[24] Sarwat, F.; Ul Qader, S. A.; Aman, A.; Ahmed, N., Production \& Characterization of a Unique Dextran from an Indigenous Leuconostoc mesenteroides CMG713. Int. J. Biol. Sci. 2008, 4 (6), 379-386.

[25] Antoniou, E.; Tsianou, M., Solution properties of dextran in water and in formamide. J. Appl. Polym. Sci. 2012, 125 (3), 1681-1692.

[26] Hornig, S.; Bunjes, H.; Heinze, T., Preparation and characterization of nanoparticles based on dextran-drug conjugates. J. Colloid Interface Sci. 2009, 338 (1), 56-62.

[27] Liebert, T.; Wotschadlo, J.; Laudeley, P.; Heinze, T., Meltable Dextran Esters As Biocompatible and Functional Coating Materials. Biomacromolecules 2011, 12 (8), 3107-3113.

[28] Hornig, S.; Heinze, T., Nanoscale structures of dextran esters. Carbohydr. Polym. 2007, 68 (2), 280-286.

[29] Kaewprapan, K.; Baros, F.; Marie, E.; Inprakhon, P.; Durand, A., Macromolecular surfactants synthesized by lipase-catalyzed transesterification of dextran with vinyl decanoate. Carbohydr. Polym. 2012, 88 (1), 313-320.

[30] Mergy, J.; Fournier, A.; Hachet, E.; Auzely-Velty, R., Modification of polysaccharides via thiol-ene chemistry: A versatile route to functional biomaterials. $J$ Polym Sci Pol Chem 2012, 50 (19), 4019-4028.

[31] Hiemstra, C.; van der Aa, L. J.; Zhong, Z.; Dijkstra, P. J.; Feijen, J., Novel in situ forming, degradable dextran hydrogels by Michael addition chemistry: Synthesis, rheology, and degradation. Macromolecules 2007, 40 (4), 1165-1173. 
[32] Hiemstra, C.; van der Aa, L. J.; Zhong, Z. Y.; Dijkstra, P. J.; Feijen, J., Rapidly in situ-forming degradable hydrogels from dextran thiols through michael addition. Biomacromolecules 2007, 8 (5), 1548-1556.

[33] Elschner, T.; Wondraczek, H.; Heinze, T., Syntheses and detailed structure characterization of dextran carbonates. Carbohydr. Polym. 2013, 93 (1), 216-223.

[34] Wondraczek, H.; Elschner, T.; Heinze, T., Synthesis of highly functionalized dextran alkyl carbonates showing nanosphere formation. Carbohydr. Polym. 2011, 83 (3), 1112-1118.

[35] Chiang, W.-H.; Lan, Y.-J.; Huang, Y.-C.; Chen, Y.-W.; Huang, Y.-F.; Lin, S.-C.; Chern, C.-S.; Chiu, H.-C., Multi-scaled polymersomes from self-assembly of octadecanol-modified dextrans. Polymer 2012, 53 (11), 2233-2244.

[36] Cui, L. N.; Cohen, J. A.; Broaders, K. E.; Beaudette, T. T.; Frechet, J. M. J., Mannosylated Dextran Nanoparticles: A pH-Sensitive System Engineered for Immunomodulation through Mannose Targeting. Bioconj. Chem. 2011, 22 (5), 949-957. [37] Ramirez, J. C.; Sanchezchaves, M.; Arranz, F., Dextran functionalized by 4nitrophenyl carbonate groups aminolysis reactions. Angew. Makromol. Chem. 1995, 225, 123-130.

[38] Jin, R.; Moreira Teixeira, L. S.; Dijkstra, P. J.; Zhong, Z.; van Blitterswijk, C. A.; Karperien, M.; Feijen, J., Enzymatically crosslinked dextran-tyramine hydrogels as injectable scaffolds for cartilage tissue engineering. Tissue Eng Part A 2010, 16 (8), 2429-2440.

[39] Jin, R.; Teixeira, L. S. M.; Dijkstra, P. J.; van Blitterswijk, C. A.; Karperien, M.; Feijen, J., Chondrogenesis in injectable enzymatically crosslinked heparin/dextran hydrogels. J. Controlled Release 2011, 152 (1), 186-195.

[40] Jin, R.; Teixeira, L. S. M.; Dijkstra, P. J.; van Blitterswijk, C. A.; Karperien, M.; Feijen, J., Enzymatically-crosslinked injectable hydrogels based on biomimetic dextranhyaluronic acid conjugates for cartilage tissue engineering. Biomaterials 2010, 31 (11), 3103-3113.

[41] Li, H.; Niu, R.; Yang, J.; Nie, J.; Yang, D., Photocrosslinkable tissue adhesive based on dextran. Carbohydr. Polym. 2011, 86 (4), 1578-1585.

[42] Wang, T.; Nie, J.; Yang, D., Dextran and gelatin based photocrosslinkable tissue adhesive. Carbohydr. Polym. 2012, 90 (4), 1428-1436. 
[43] Nouvel, C.; Raynaud, J.; Marie, E.; Dellacherie, E.; Six, J. L.; Durand, A., Biodegradable nanoparticles made from polylactide-grafted dextran copolymers. $J$. Colloid Interface Sci. 2009, 330 (2), 337-343.

[44] Lemechko, P.; Renard, E.; Guezennec, J.; Simon-Colin, C.; Langlois, V., Synthesis of dextran-graft-PHBHV amphiphilic copolymer using click chemistry approach. React. Funct. Polym. 2012, 72 (8), 487-494.

[45] Heinze, T.; Michealis, N.; Hornig, S., Reactive polymeric nanoparticles based on unconventional dextran derivatives. Eur. Polym. J. 2007, 43 (3), 697-703.

[46] Klinger, D.; Landfester, K., Enzymatic- and light-degradable hybrid nanogels: Crosslinking of polyacrylamide with acrylate-functionalized Dextrans containing photocleavable linkers. J Polym Sci Pol Chem 2012, 50 (6), 1062-1075.

[47] Houga, C.; Le Meins, J. F.; Borsali, R.; Taton, D.; Gnanou, Y., Synthesis of ATRPinduced dextran-b-polystyrene diblock copolymers and preliminary investigation of their self-assembly in water. Chem. Commun. 2007, 29, 3063-3065.

[48] Houga, C.; Giermanska, J.; Lecommandoux, S.; Borsali, R.; Taton, D.; Gnanou, Y.; Le Meins, J. F., Micelles and Polymersomes Obtained by Self-Assembly of Dextran and Polystyrene Based Block Copolymers. Biomacromolecules 2009, 10 (1), 32-40.

[49] Liu, J. Y.; Zhang, L. M., Preparation of a polysaccharide-polyester diblock copolymer and its micellar characteristics. Carbohydr. Polym. 2007, 69 (1), 196-201.

[50] Li, B. G.; Wang, Q.; Wang, X.; Wang, C. Z.; Jiang, X. Q., Preparation, drug release and cellular uptake of doxorubicin-loaded dextran-b-poly(epsilon-caprolactone) nanoparticles. Carbohydr. Polym. 2013, 93 (2), 430-437.

[51] Schatz, C.; Louguet, S.; Le Meins, J. F.; Lecommandoux, S., Polysaccharideblock-polypeptide copolymer vesicles: towards synthetic viral capsids. Angew. Chem. Int. Ed. Engl. 2009, 48 (14), 2572-2575.

[52] Sun, H. L.; Guo, B. N.; Li, X. Q.; Cheng, R.; Meng, F. H.; Liu, H. Y.; Zhong, Z. Y., Shell-Sheddable Micelles Based on Dextran-SS-Poly(epsilon-caprolactone) Diblock Copolymer for Efficient Intracellular Release of Doxorubicin. Biomacromolecules 2010, 11 (4), 848-854.

[53] Novoa-Carballal, R.; Mueller, A. H. E., Synthesis of polysaccharide-b-PEG block copolymers by oxime click. Chem. Commun. 2012, 48 (31), 3781-3783. 
[54] Hernandez, O. S.; Soliman, G. M.; Winnik, F. M., Synthesis, reactivity, and pHresponsive assembly of new double hydrophilic block copolymers of carboxymethyldextran and poly(ethylene glycol). Polymer 2007, 48 (4), 921-930. [55] Aumelas, A.; Serrero, A.; Durand, A.; Dellacherie, E.; Leonard, M., Nanoparticles of hydrophobically modified dextrans as potential drug carrier systems. Colloids Surf., B 2007, 59 (1), 74-80.

[56] Mehvar, R.; Dann, R. O.; Hoganson, D. A., Kinetics of hydrolysis of dextranmethylprednisolone succinate, a macromolecular prodrug of methylprednisolone, in rat blood and liver lysosomes. J. Controlled Release 2000, 68 (1), 53-61.

[57] Zalipsky, S.; Harris, J. M. In Poly(Ethylene Glycol): Chemistry and Biological Applications; Harris, J. M., Zalipsky, S., Eds. 1997; Vol. 680, p 1-13.

[58] Chapman, A. P., PEGylated antibodies and antibody fragments for improved therapy: a review. Adv. Drug Del. Rev. 2002, 54 (4), 531-545.

[59] Hinds, K. D.; Kim, S. W., Effects of PEG conjugation on insulin properties. Adv. Drug Del. Rev. 2002, 54 (4), 505-530.

[60] Kinstler, O.; Molineux, G.; Treuheit, M.; Ladd, D.; Gegg, C., Mono-N-terminal poly(ethylene glycol)-protein conjugates. Adv. Drug Del. Rev. 2002, 54 (4), 477-485.

[61] Sato, H., Enzymatic procedure for site-specific pegylation of proteins. Adv. Drug Del. Rev. 2002, 54 (4), 487-504.

[62] Wang, Y. S.; Youngster, S.; Grace, M.; Bausch, J.; Bordens, R.; Wyss, D. F., Structural and biological characterization of pegylated recombinant interferon alpha- $2 \mathrm{~b}$ and its therapeutic implications. Adv. Drug Del. Rev. 2002, 54 (4), 547-570.

[63] Roberts, M. J.; Bentley, M. D.; Harris, J. M., Chemistry for peptide and protein PEGylation. Adv. Drug Del. Rev. 2002, 54 (4), 459-476.

[64] Sawhney, A. S.; Pathak, C. P.; Hubbell, J. A., Bioerodible hydrogels based on photopolymerized poly(ethylene glycol)-co-poly(alpha-hydroxy acid) diacrylate macromers. Macromolecules 1993, 26 (4), 581-587.

[65] Buwalda, S. J.; Calucci, L.; Forte, C.; Dijkstra, P. J.; Feijen, J., Stereocomplexed 8armed poly(ethylene glycol)-poly(lactide) star block copolymer hydrogels: Gelation mechanism, mechanical properties and degradation behavior. Polymer 2012, 53 (14), 2809-2817. 
[66] Zhang, Z. F.; Loebus, A.; de Vicente, G.; Ren, F.; Arafeh, M.; Ouyang, Z. F.; Lensen, M. C., Synthesis of Poly(ethylene glycol)-based Hydrogels via Amine-Michael Type Addition with Tunable Stiffness and Postgelation Chemical Functionality. Chem. Mater. 2014, 26 (12), 3624-3630.

[67] Chenault, H. K.; Bhatia, S. K.; DiMaio, W. G.; Vincent, G. L.; Camacho, W.; Behrens, A., Sealing and Healing of Clear Corneal Incisions with an Improved Dextran Aldehyde-PEG Amine Tissue Adhesive. Curr. Eye Res. 2011, 36 (11), 997-1004.

[68] Kirschner, C. M.; Alge, D. L.; Gould, S. T.; Anseth, K. S., Clickable, Photodegradable Hydrogels to Dynamically Modulate Valvular Interstitial Cell Phenotype. Adv. Healthc. Mater. 2014, 3 (5), 649-657.

[69] Kirchhof, S.; Strasser, A.; Wittmann, H. J.; Messmann, V.; Hammer, N.; Goepferich, A. M.; Brandl, F. P., New insights into the cross-linking and degradation mechanism of Diels-Alder hydrogels. J. Mater. Chem. 2015, 3 (3), 449-457.

[70] Fan, M.; Zhang, Z. W.; Mao, J. H.; Tan, H. P., Injectable Multi-Arm Poly(ethylene glycol)/Hyaluronic Acid Hydrogels for Adipose Tissue Engineering. Journal of Macromolecular Science Part a-Pure and Applied Chemistry 2015, 52 (5), 345-352.

[71] Hiemstra, C.; Zhong, Z. Y.; Li, L. B.; Dijkstra, P. J.; Feijen, J., In-situ formation of biodegradable hydrogels by stereocomplexation of PEG-(PLLA)(8) and PEG(PDLA)(8) star block copolymers. Biomacromolecules 2006, 7 (10), 2790-2795.

[72] Hiemstra, C.; Zhong, Z.; Van Tomme, S. R.; van Steenbergen, M. J.; Jacobs, J. J. L.; Den Otter, W.; Hennink, W. E.; Feijen, J., In vitro and in vivo protein delivery from in situ forming poly(ethylene glycol)-poly(lactide) hydrogels. J. Controlled Release 2007, 119 (3), 320-327.

[73] Nagahama, K.; Fujiura, K.; Enami, S.; Ouchi, T.; Ohya, Y., Irreversible temperature-responsive formation of high-strength hydrogel from an enantiomeric mixture of starburst triblock copolymers consisting of 8-arm PEG and PLLA or PDLA. J. Polym. Sci., Part A: Polym. Chem. 2008, 46 (18), 6317-6332.

[74] Nagahama, K.; Ouchi, T.; Ohya, Y., Temperature-induced hydrogels through selfassembly of cholesterol-substituted star PEG-b-PLLA copolymers: An injectable scaffold for tissue engineering. Adv. Funct. Mater. 2008, 18 (8), 1220-1231. 
[75] Buwalda, S. J.; Dijkstra, P. J.; Feijen, J., Poly(ethylene glycol)-poly(L-lactide) star block copolymer hydrogels crosslinked by metal-ligand coordination. Journal of Polymer Science Part a-Polymer Chemistry 2012, 50 (9), 1783-1791.

[76] van de Manakker, F.; van der Pot, M.; Vermonden, T.; van Nostrum, C. F.; Hennink, W. E., Self-assembling hydrogels based on beta-cyclodextrin/cholesterol inclusion complexes. Macromolecules 2008, 41 (5), 1766-1773.

[77] van de Manakker, F.; Braeckmans, K.; el Morabit, N.; De Smedt, S. C.; van Nostrum, C. F.; Hennink, W. E., Protein-Release Behavior of Self-Assembled PEGbeta-Cyclodextrin/PEG-Cholesterol Hydrogels. Adv. Funct. Mater. 2009, 19 (18), 29923001.

[78] Hiemstra, C.; Zhou, W.; Zhong, Z.; Wouters, M.; Feijen, J., Rapidly in situ forming biodegradable robust hydrogels by combining stereocomplexation and photopolymerization. J. Am. Chem. Soc. 2007, 129 (32), 9918-9926. 
Chapter 2 


\section{Chapter 3}

Cartilage adhesive and mechanical properties of enzymatically crosslinked polysaccharide tyramine conjugate hydrogels*
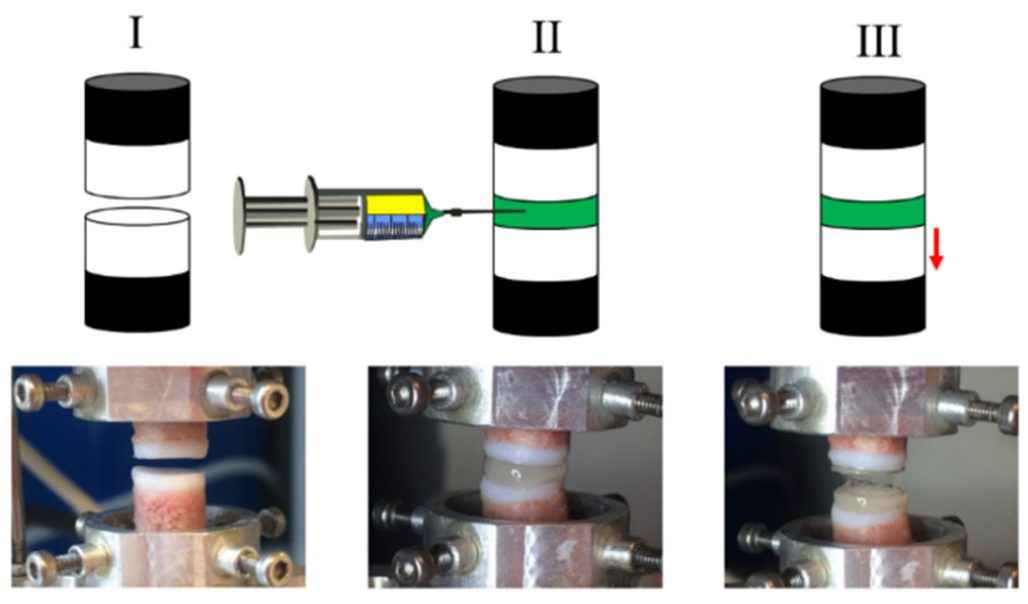

* This chapter has been published: Wang, R.; Leber, N.; Buhl, C.; Verdonschot, N.; Dijkstra, P. J.; Karperien, M.,. Polym. Adv. Technol. 2014, 25 (5), 568-574. 


\section{Abstract}

Using a home built tensile tester adhesion and mechanical properties of injectable enzymatically crosslinkable hydrogels were determined by placing the hydrogels in between cartilage surfaces. Dextran tyramine (Dex-TA) and hyaluronic acid tyramine (HA-TA) conjugates as well as a 50/50 composite material of these polysaccharide conjugates were tested. To integrate the injectable hydrogels with the cartilage tissue pretreatment of the tissue with a dextran tyramine conjugate solution strongly improved the adhesion. Only failure of the crosslinked hydrogel was observed and not at the hydrogel tissue interface. Moduli of a Dex-TA hydrogel are higher than those of a HATA hydrogel whereas the ultimate strain of the HA-TA hydrogel was at least 3 times higher. The Dex-TA/HA-TA hydrogel has similar storage and elastic moduli as the DexTA gel and also an ultimate strain of $\sim 30 \%$, similarly as found for the HA-TA gel. The controlled biodegradability and gelation time of the Dex-TA/HA-TA hydrogel, the developed method for strong tissue adhesion of the gel particularly in comparison to fibrin glue makes this material applicable as an injectable hydrogel for tissue regeneration applications. 


\subsection{Introduction}

Cartilage damage due to trauma, wear and diseases like osteoarthitis are the most common reasons for joint disability. The limited regenerative capacity of cartilage mostly leads to further degeneration of the tissue in time. Treatment of degenerated cartilage nowadays can be performed by techniques like microfracture or mosaicplasty, however results are not satisfying. With a population that becomes increasingly active and older it is expected that the prevalence of cartilage damage will increase and development of new strategies for cartilage repair become necessary [1].

Hydrogels, 3D elastic networks, mimic hydrated native cartilage tissue and are considered suitable scaffolds for cartilage tissue engineering. Although preformed hydrogels may be applied their main disadvantage is the need for invasive surgical interventions. In-situ forming hydrogels, also called injectable hydrogels, have attracted attention in recent years since they offer various advantages. In contrast to preformed hydrogels, they can be applied in a minimally invasive surgical procedure, can fill irregular-shaped defects and can be co-injected with cells and bioactive molecules mixed in the precursor solution. Approaches currently used to fill defects focus on cell free hydrogels or hydrogels containing autologous cells. Although much progress has been made in the development of new materials for cartilage regeneration several considerations have to be made to effect new cartilage formation in a proper way revealing the complex nature of this tissue [2-5].

In a review paper of Khan et al. [6] several factors impeding repair of cartilage were described. Cell death at the hydrogel-tissue interface, impaired differentiation of chondrocytes in constructs containing cells, donor age related effects of cells used to repair the defect, and pretreatment of the interface with collagenase to improve chondrocyte outgrowth may all contribute to implant failure. In this respect, the success of the integration of graft materials with the host tissue is dependent on the viability of chondrocytes at the edges of the cartilage. Also maintaining the chondrocyte phenotype is important in the function and deposition of a new matrix and contributes to the success of the formation of new cartilage and integration of new and existing tissue. Inhibition of chondrocyte cell death and stimulation of cell density together with facilitating cell migration are considered to contribute to cartilage healing. Adhesive 
properties of implants like injectable hydrogels most likely critically determines integration of the gel with the cartilage [7]. In order to clinically transform the use of injectable hydrogels in cartilage repair, it is necessary to integrate the hydrogel in a stable way in the surrounding tissue. Although some studies have addressed tissue integration only a limited number of studies are related to the firm adhesion of hydrogels to the surrounding tissue. The group of Elisseeff [8] has performed most work in this area and translation of their materials to clinic is in progress [9]. To firmly adhere hydrogels to the existing tissue they used a strategy by first priming the tissue with reactive groups that subsequently can be co-polymerized with a photopolymerizable hydrogel precursor solution. They used functionalized chondroitin sulfate with methacrylate groups and aldehyde groups. Aldehyde groups were introduced by oxidizing part of the rings. This material was used as a primer. By formation of Schiff-base groups upon reaction of the aldehyde groups of the polysaccharide with free amine groups from e.g. lysine residues of the collagen the chondroitin sulfate conjugate was covalently attached to the tissue. Upon filling the defect with the photopolymerizable hydrogel precursor the acrylate groups of the chondroitin sulfate were co-crosslinked thereby attaching the hydrogel to the tissue interface. Tensile and shear forces applied to the tissue-gel constructs revealed failure in the hydrogel showing the proper fixation of the hydrogel at the interface.

The deposition of a new matrix by cells in hydrogels may well contribute to the adhesion of the gel to the tissue. Erickson et al. [10] studied the integration and compressive properties of a photocrosslinkable methacrylated hyaluronic acid hydrogel containing mesenchymal stem cells in cartilage explants. It was shown that the hydrogel-MSC's constructs afforded higher equilibrium moduli in a push out test than materials without cells. It was hypothesized that the deposition of new matrix contributed to the better integration and thereby adhesion of the hydrogel to the cartilage. Maher et al. [11] prepared gels containing chondrocytes using Puramatrix ${ }^{\mathrm{TM}}$. Puramatrix $^{\mathrm{TM}}$ is a peptide-based hydrogel having a nanometer scale fibrous structure. By addition of transforming growth factor- $\beta 3$ (TGF- $\beta 3$ ), integration of the gels with cartilage appears to be enhanced as measured by a push out method. Although no explanation was presented, it is believed that matrix deposition at the interface can be regarded as the main reason for the improved adhesion. 
Recently an injectable hydrogel has been described which is based on the use of oxidized carboxymethyl cellulose, borax and gelatin. Such a gel can be applied to cartilage defects using a double syringe. In this method the borax provided linkages with remaining hydroxyl groups while the aldehyde groups on the partially oxidized polysaccharide were used to induce crosslinking via Schiff base formation with the gelatin free amine groups [12]. The in situ gelating material was tested for its integration with goat cartilage explants. Good integration was shown and the adhesion to cartilage was determined via its burst strength. The formation of Schiff base groups between collagen fibers located at the defect interface and oxidized carboxymethyl cellulose in the hydrogel was reported a reason for the adhesive nature of the hydrogel.

In previous research we have shown that in-situ forming hydrogels can be prepared by the horseradish peroxidase-mediated (co)-crosslinking of dextran-tyramine (DexTA) and hyaluronic acid-tyramine (HA-TA) conjugates [13-15]. Initial results on placing such in-situ forming hydrogels into a cartilage defect indicated adhesion to the surrounding tissue. We hypothesized that the reaction results in covalent binding of the hydroxyphenyl groups in the polysaccharide tyramine conjugate to collagen tyrosine functional groups in the cartilage matrix. In this paper, we describe a method to measure the adhesion strength of in-situ forming injectable and enzymatically crosslinkable hydrogels to cartilage tissue. We used a sandwich model in which the hydrogels were injected in between two cartilage explants. Applying a tensile force, disruption in the hydrogels or at the hydrogels cartilage interface could be determined. Moreover, this method also allowed for measuring the hydrogel's mechanical properties. These properties were compared to mechanical properties of the hydrogels by rheology.

\subsection{Materials and methods}

Dextran $\left(\mathrm{M}_{\mathrm{w}}=15\right.$ to $\left.25 \mathrm{~kg} / \mathrm{mol}\right)$ was obtained from Sigma-Aldrich and dried by lyophilization. Hyaluronic acid sodium salt $\left(\mathrm{M}_{\mathrm{w}}=15\right.$ to $\left.25 \mathrm{~kg} / \mathrm{mol}\right)$ was purchased from CPN-shop. Fibrin gel with 2 components (Tissucol Duo 500) was purchased from Baxter AG. N-hydroxysuccinimide (NHS), tyramine, p-nitrophenyl chloroformate (PNC), anhydrous dimethylformamide (DMF), pyridine (anhydrous), hydrogen peroxide $\left(\mathrm{H}_{2} \mathrm{O}_{2}, 30\right.$ wt \% in $\left.\mathrm{H}_{2} \mathrm{O}\right)$, lithium chloride, horseradish peroxidase (HRP, 253 
purpurogallin units/mg solid) were obtained from Sigma-Aldrich and used without further purification. N-Ethyl-N'-(3-dimethylaminopropyl) carbodiimide hydroxide (EDAC) was obtained from Fluka. Phosphate buffered saline (PBS, pH 7.4) was purchased from B. Braun Co. The dextran-tyramine conjugate (Dex-TA) and hyaluronic acid tyramine conjugate (HA-TA) were synthesized using slightly modified procedures as described previously [14].

\subsubsection{Synthesis}

Dextran-tyramine conjugate (Dex-TA): Typically, dextran $(3.0 \mathrm{~g}, 56.3 \mathrm{mmol} \mathrm{OH}$ groups) was dissolved in dimethyl formamide (DMF, $120 \mathrm{~mL}$, containing $2.4 \mathrm{~g}$ of $\mathrm{LiCl}$ ) at $90{ }^{\circ} \mathrm{C}$ under nitrogen. After the dextran was dissolved, the mixture was cooled and thermostated at $0^{\circ} \mathrm{C}$. Pyridine $(1.5 \mathrm{ml}, 18.6 \mathrm{mmol})$ and 4-nitrophenyl chloroformate (PNC, $2.6 \mathrm{~g}, 12.9 \mathrm{mmol}$ ) were added to the solution while stirring. The reaction was conducted for $1 \mathrm{~h}$, and the product (denoted as Dex-PNC) was precipitated in cold ethanol, filtered and washed with ethanol and diethyl ether. DS ( ${ }^{1} \mathrm{H}$ NMR): 24 . Yield: $3.7 \mathrm{~g}(99 \%) .{ }^{1} \mathrm{H}$ NMR (DMSO-d $\left.{ }_{6}\right): 3.0-4.0$ (m, dextran glucosidic protons), 4.4-5.8 (m, dextran anomeric protons and $\mathrm{OH}), 7.58$ and 8.32 (d, aromatic protons).

Subsequently, Dex-PNC DS 24 (3.7 g, $4.4 \mathrm{mmol}$ of 4-nitrophenyl carbonate groups) was dissolved in $57 \mathrm{~mL}$ of DMF and tyramine (1.2 $\mathrm{g}, 8.9 \mathrm{mmol})$ was added under nitrogen. The reaction was conducted for $1 \mathrm{~h}$. The product was precipitated in cold ethanol, filtered and washed with ethanol and diethyl ether. The Dex-TA conjugate was purified by ultrafiltration (MWCO 3000) against deionized water and isolated after lyophilization. DS ( ${ }^{1} \mathrm{H}$ NMR): 12. Yield: $2.7 \mathrm{~g}(82 \%) .{ }^{1} \mathrm{H}$ NMR $\left(\mathrm{D}_{2} \mathrm{O}\right): \delta 2.75$ and 3.05 (m, $-\mathrm{CH}_{2}-\mathrm{CH}_{2}-$ ), 3.3-4.1 (m, dextran glucosidic protons), 4.2-5.6 (s, dextran anomeric protons), 6.86 and 7.17 (d, tyramine aromatic protons).

Hyaluronic acid-tyramine conjugate (HA-TA): Sodium hyaluronate $(1.0 \mathrm{~g})$ was dissolved in $20 \mathrm{~mL}$ of MES buffer (0.1 M, pH 6.0), EDAC (240 mg, $1.25 \mathrm{mmol})$ and NHS (144 mg, $1.25 \mathrm{mmol}$ ) both separately dissolved in $5.0 \mathrm{~mL}$ of MES buffer were added. After $30 \mathrm{~min}$ of stirring a solution of tyramine $(342 \mathrm{mg}, 2.50 \mathrm{mmol})$ in $6 \mathrm{~mL}$ of DMF was added and the mixture was stirred for 3 days. The mixture was neutralized with $1 \mathrm{M} \mathrm{NaOH}$ and ultrafiltrated (MWCO 1000), first with PBS and then MilliQ- 
water. The resultant HA-Tyr conjugate was obtained after lyophilization as a white foam. Yield: $0.72 \mathrm{~g}(71 \%)$. The degree of substitution was DS (UV/Vis): 2.2. ${ }^{1} \mathrm{H}$ NMR (400 MHz, $\mathrm{D}_{2} \mathrm{O}$ ): $\delta 1.93$ (s, methyl protons); 2.4-2.8 (m, polysaccharide ring protons); 3.2-4.1 ( $\mathrm{m}$, polysaccharide ring protons); 4.2-4,6 (d, 1H, polysaccharide ring protons); $4.69\left(\mathrm{~s}, \mathrm{H}_{2} \mathrm{O}\right) ; 6.86$ and 7.17 (d, tyramine aromatic protons).

\subsubsection{Characterization}

${ }^{1} \mathrm{H}-\mathrm{NMR}:{ }^{1} \mathrm{H}$ (400MHz) NMR spectra were recorded using a Bruker AVANCE III 400 $\mathrm{MHz}$ (Nanobay) spectrometer. Polymers were dissolved in $\mathrm{D}_{2} \mathrm{O}$ or DMSO at a concentration of $0.020 \mathrm{~g} / \mathrm{mL}$. The degree of substitution (DS) of tyramine groups was calculated using the integral ratio between the aromatic protons of the tyramine groups and anomeric and $\mathrm{OH}$ protons of the polysaccharide backbone.

UV/Vis: The degree of substitution of tyramine residues in Dex-TA and HA-TA conjugates was determined by an UV measurement. The HA-TA conjugate was dissolved in PBS at a concentration of $5 \mu \mathrm{g} / \mathrm{mL}$ and the absorbance was measured at $275 \mathrm{~nm}$ using a Cary 300 Bio spectrometer (Varian). The absorbance was correlated to the DS of tyramine groups in the HA-TA using a calibration curve from tyramine in PBS.

Rheological analysis: Rheological experiments were carried out with a MCR 301 rheometer (Anton Paar) using parallel plates $\left(25 \mathrm{~mm}\right.$ diameter, $\left.0^{\circ}\right)$ configuration at 37 ${ }^{\circ} \mathrm{C}$ in the oscillatory mode. In a typical experiment a solution of HRP $(25 \mu \mathrm{L}, 150$ units $/ \mathrm{mL}$ stock solution) and $\mathrm{H}_{2} \mathrm{O}_{2}(25 \mu \mathrm{L}, 0.3 \%$ stock solution) were mixed and added to the polymer dissolved in PBS ( $200 \mu \mathrm{L}, 10 \mathrm{wt} \%)$. The resulting gel was placed at the ground plate and the upper plate was lowered to a measuring gap size of $0.3 \mathrm{~mm}$, and the measurement was started. To prevent water evaporation, a layer of oil was introduced around the polymer sample. The storage $\left(\mathrm{G}^{\prime}\right)$ and loss $\left(\mathrm{G}^{\prime \prime}\right)$ modulus were recorded using a strain of $1 \%$ and a frequency of $1 \mathrm{~Hz}$.

Tensile testing: A tensile test set-up with a $9 \mathrm{~N}$ load cell was used to measure the material response on tensile forces. Bovine cartilage explants were obtained from fresh bovine knees using a Trukor drill $(\varnothing=8 \mathrm{~mm})$ sleeve. In a typical experiment, a $10 \mathrm{wt} \%$ Dex-TA hydrogel was injected between two bovine cartilage pieces. Mixing was 
achieved using a Mixpac ${ }^{(\mathrm{TM})}$ double syringe. A Dex-TA polymer solution $(20 \mathrm{wt} \%$ in PBS) was mixed with an equal volume of HRP (150 unit $/ \mathrm{mL}$ stock solution in PBS) and placed in one of the chambers. To the other chamber of the double syringe was added a mixture of the Dex-TA polymer solution (20 wt \% in PBS) and an equal volume of $\mathrm{H}_{2} \mathrm{O}_{2}$ ( $0.3 \mathrm{wt} \%$ stock solution in PBS). Gels were formed in-situ between the cartilage pieces by injection. After $5 \mathrm{~min}$ the cartilage pieces were pulled apart at a uniform speed of $0.1 \mathrm{~mm} / \mathrm{s}$. As control fibrin gels (Tissucol, Baxter), were applied in a similar way. Cartilage surfaces were either non-treated or pretreated with a 10 wt \% Dex-TA polymer solution in PBS for $5 \mathrm{~min}$ before injecting the in-situ forming hydrogels.

\subsection{Results and discussion}

\subsubsection{Synthesis}

The synthesis of the dextran-tyramine (Dex-TA) and hyaluronic acid-tyramine (HATA) conjugates (Figure 3.1) was performed in an analogues way as described previously with some modifications. The activation of dextran hydroxyl functional groups with 4-nitrophenyl chloroformate was performed according to a paper of Schacht and coworkers [16]. The reaction appears highly sensitive to impurities like amines present in the solvent (dimethyl formamide) and moisture. Moreover, a reaction time of $1 \mathrm{~h}$ appeared sufficient and longer reaction times decreased the number of activated hydroxyl groups. The subsequent reaction with tyramine affords the Dex-TA conjugate through the formation of carbamate groups. For the intended application as an injectable hydrogel using the enzymatic crosslinking by horseradish peroxidase and hydrogen peroxide of phenolic groups, the number of tyramine groups on the dextran backbone needs to be within certain values. Low degrees of substitution (DS $<10$ ) do give slow gelation applying a conjugate concentration of $10 \mathrm{wt} \%$. The upper limit in the DS of tyramine groups in Dex-TA with an average molecular weight of 16000 is approximately 17. At higher degrees of substitution the conjugate will become insoluble in phosphate buffered saline (PBS). Within the range of DS values given above, the gelation time can be tuned to approximately 20 seconds by using appropriate HRP and 
$\mathrm{H}_{2} \mathrm{O}_{2}$ concentrations. Such gelation times are highly suitable for the conjugate to be applied as an injectable hydrogel.

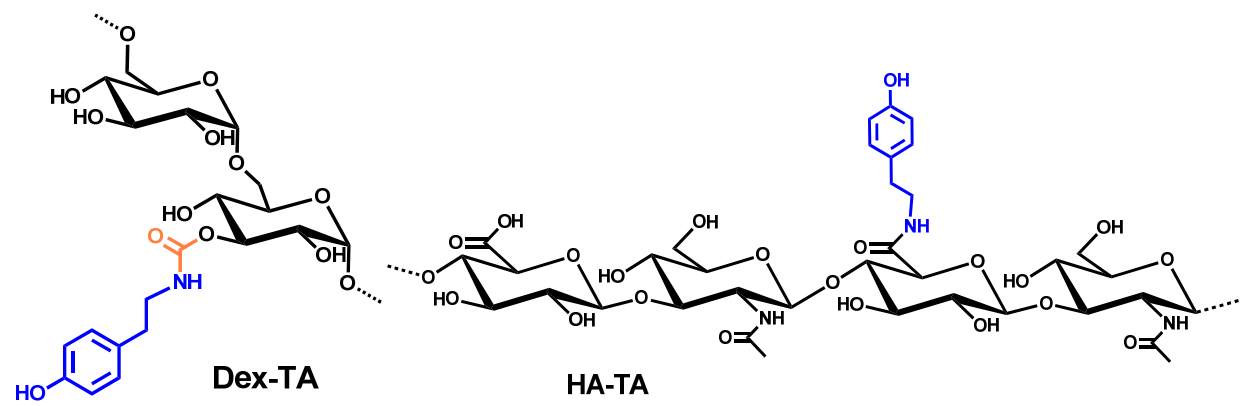

Figure 3.1 Chemical structure of the dextran tyramine conjugate (Dex-TA) and hyaluronic acid tyramine conjugate (HA-TA).
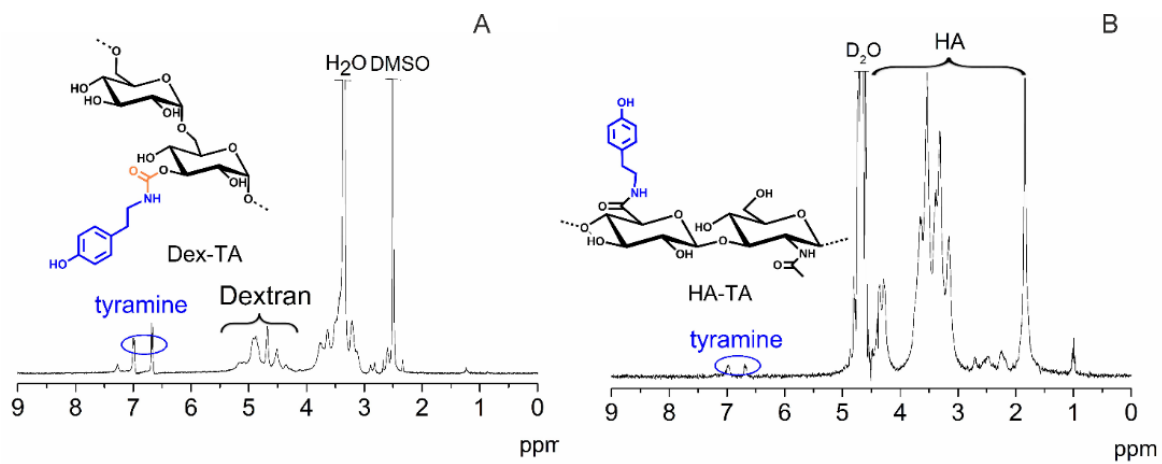

Figure 3.2 ${ }^{1} \mathrm{H}-\mathrm{NMR}$ spectra (400 MHz) of (A) Dextran tyramine conjugate (Dex-TA) and (B) Hyaluronic acid tyramine conjugate (HA-TA).

In a recent paper we described the synthesis of a HA-TA conjugate using the wellknown method of carboxylic acid activation by ethyl diaminopropyl carbodiimide (EDC) and N-hydroxy succinimide (NHS) and subsequent reaction with tyramine. In this one pot synthesis using an excess of activation reagents and tyramine, a degree of substitution of 2 to 3 was obtained. The degree of substitution of the HA-TA was determined both by ${ }^{1} \mathrm{HNMR}\left(\mathrm{D}_{2} \mathrm{O}\right)$ (Figure 3.2) and UV-Vis at $275 \mathrm{~nm}$ using a calibration curve of tyramine in MilliQ water. The values obtained by NMR (DS = 2.0) and UV-Vis (DS = 2.2) are similar. This means that approximately $5 \%$ of the carboxylic acid groups have been substituted with a tyramine group. Despite this 
apparent low degree of substitution, fast gelation within 20 s using HRP and $\mathrm{H}_{2} \mathrm{O}_{2}$ concentrations similar as given above takes place.

\subsubsection{Adhesion tests}

An important issue in the application of an in-situ forming hydrogel to fill cartilage defects is the integration and adhesion of the gel to the surrounding tissue. In previous research we showed that using a double syringe as an injection system homogeneous enzymatically crosslinked Dex-TA gels could be applied to fill defects made in fresh bovine explants [15]. SEM and Raman analysis showed that the gel even at irregular sites filled the defects. The adhesion of the Dex-TA gels was explained by the cocrosslinking of collagen tyrosine groups with the tyramine groups on the polysaccharide backbone.

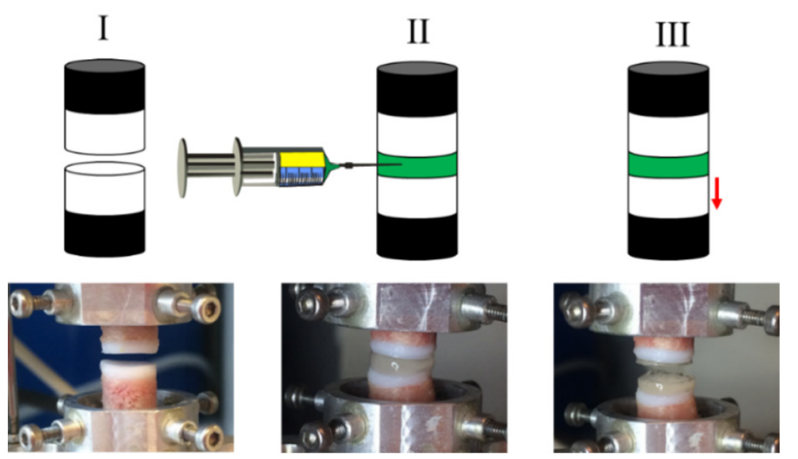

Figure 3.3 Schematic representation of the tensile tests performed. I: cylindrical cartilage specimens were placed in the holders of the tensile tester; II: hydrogels were injected in the gap; III: disrupted hydrogel after loading.

In this study we used a home built tensile tester to quantify the adhesion of the gels to cartilage. Fresh bovine knees were dissected and the synovial fluid drained. Using a Trukor sleeve, cylindrical specimens $(\varnothing=8 \mathrm{~mm})$ were taken from the cartilage up to the subchondral bone. The tissue was kept moist by regularly rinsing with PBS. The tester consists of a $9 \mathrm{~N}$ load cell and two holders of which the lower one can be pushed downwards. Two cylindrical specimens $(\varnothing=8 \mathrm{~mm})$ were taken from bovine knees and 
mounted in the holders (Figure 3.3). The hydrogel precursor solutions were injected in between the two specimens (gap size of $3 \mathrm{~mm}$ ) using a double syringe. The gel was formed almost instantaneously and after 5 min curing a tensile test experiment was performed at a measured rate of $100 \mu \mathrm{m} / \mathrm{s}$. From the measured forces corresponding to a certain position a stress-strain curve was determined.

However, occasionally, it could be observed that the shrinkage of the Dex-TA gel upon crosslinking leads to detachment of the gel from the tissue. Apparently no integration or insufficient integration of the Dex-TA with the cartilage takes place and generally this could clearly be observed visually. It thus appears that a proper balance is needed between gelation time and time needed for the precursor solutions to integrate with the tissue. Detachment of a commercial fibrin gel (Tissucol Duo 500, Baxter) was also observed applying this injectable gel in between non-treated cartilage surfaces. In this case the mixing of fibrinogen and thrombin forms a gel. Several experiments using fresh bovine cartilage cylindrical specimens afforded similar stress strain curves and a typical example is presented in Figure 3.4A. In this graph the stress is given in excited force $(\mathrm{kPa})$ as a function of the corresponding tensile strain $(\%)$.
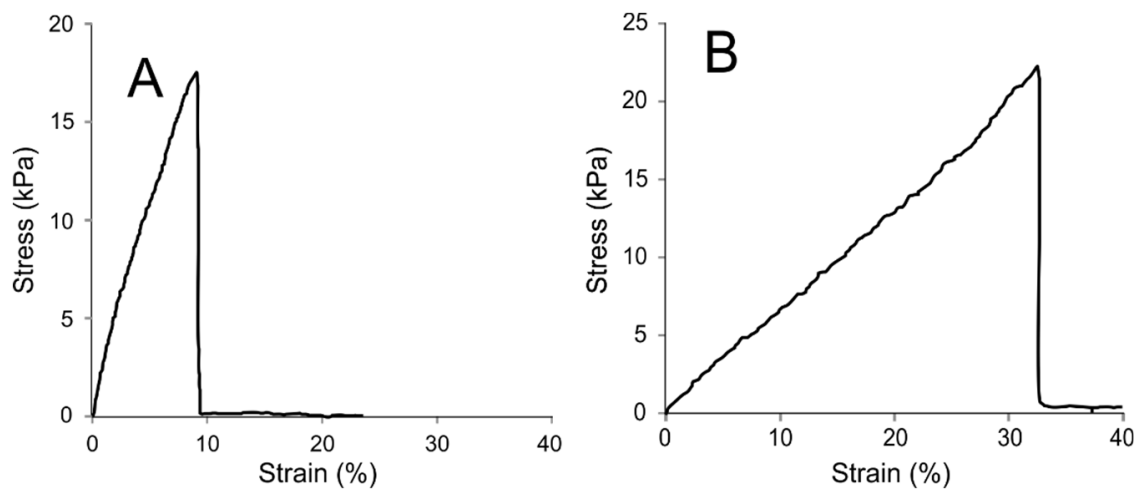

Figure 3.4 Stress-strain curves of hydrogels injected in between two cartilage specimen. A $10 \mathrm{wt} \%$ Dex-TA gel (A) and a $10 \mathrm{wt} \%$ HA-TA gel (B). The cartilage surfaces were pretreated with a $10 \mathrm{wt} \%$ Dex-TA solution for $5 \mathrm{~min}$.

In following experiments, we therefore pretreated the cartilage surface with a $10 \mathrm{wt}$ $\%$ Dex-TA solution and after $5 \mathrm{~min}$ the hydrogel precursor solutions were injection in 
the gap. The pretreatment ensured in all cases tensile tests could be performed revealing integration of the gel with the cartilage.

The stress-strain diagram visualizes the mechanical properties of the hydrogel. In all cases failure takes place in the Dex-TA hydrogel and not at the interface. As can be seen in Figure 3.4A the stress increases almost linearly with the strain applied. No clear yield point is observed and an E-modulus of $3.7 \mathrm{kPa}$ was calculated in between 0.2 and $1.0 \%$ strain. An average ultimate stress of approximately $17 \mathrm{kPa}$ and ultimate strain of $8 \%$ were found (Table 3.1).

A typical stress strain curve of an injected $10 \mathrm{wt} \%$ HA-TA gel, applying the same concentrations of HRP and $\mathrm{H}_{2} \mathrm{O}_{2}$, is presented in Figure 3.4B. Also in this case the cartilage surface was pretreated with a $10 \mathrm{wt} \%$ Dex-TA solution for $5 \mathrm{~min}$. Failure occurs in the gel and not at the interface. Compared to the Dex-TA gel the HA-TA gel shows a somewhat lower E-modulus, similar ultimate stress but a much higher strain at break.

Table 3.1 Mechanical properties of injected Dex-TA, HA-TA and Dex-TA/HA-TA $(50 / 50)$ hydrogels in between cartilage surfaces. ${ }^{a}$

\begin{tabular}{|c|c|c|c|c|c|c|}
\hline & $\begin{array}{c}\mathrm{E} \\
\text { modulus } \\
(\mathrm{kPa})\end{array}$ & $\begin{array}{c}\text { Ultimate } \\
\text { stress } \\
(\mathrm{kPa})\end{array}$ & $\begin{array}{c}\text { Ultimate } \\
\text { strain } \\
(\%)\end{array}$ & $\begin{array}{l}\text { Storage } \\
\text { modulus } \\
(\mathrm{kPa})\end{array}$ & $\begin{array}{c}\text { Loss } \\
\text { modulus } \\
(\mathrm{kPa})\end{array}$ & $\begin{array}{c}\text { Damping } \\
\text { factor }\end{array}$ \\
\hline Dex-TA $^{b}$ & $3.7 \pm 0.6$ & $17.6 \pm 0.5$ & $7.6 \pm 2.1$ & $12.2 \pm 0.04$ & $0.9 \pm 0.03$ & 0.07 \\
\hline HA-TA ${ }^{b}$ & $0.7 \pm 0.1$ & $20.8 \pm 1.7$ & $34.0 \pm 1.8$ & $3.3 \pm 0.04$ & $0.005 \pm 0.1$ & 0.002 \\
\hline $\begin{array}{c}\text { Dex-TA } \\
/ \text { HA-TA }^{\mathrm{b}} \\
(1 / 1)\end{array}$ & $3.8 \pm 1.2$ & $37.3 \pm 11$ & $30.8 \pm 6.0$ & \multirow[b]{2}{*}{$11.3 \pm 0.5$} & \multirow[b]{2}{*}{$0.3 \pm 0.02$} & \multirow[b]{2}{*}{0.03} \\
\hline $\begin{array}{c}\text { Dex-TA } \\
\text { HA-TA }^{c} \\
(1 / 1)\end{array}$ & $3.4 \pm 0.6$ & $18.2 \pm 0.4$ & $10.4 \pm 3.5$ & & & \\
\hline Fibrin $^{c}$ & 0 & -- & -- & $0.5 \pm 0.02$ & $0.007 \pm 0.1$ & 0.02 \\
\hline
\end{tabular}

${ }^{a}$ : standard deviations were determined from at least 3 independent measurements.

${ }^{\mathrm{b}}$ : the cartilage was pretreated with a $10 \mathrm{wt} \%$ Dex-TA polymer solution for $5 \mathrm{~min}$.

${ }^{c}:$ the cartilage was non-pretreated. 

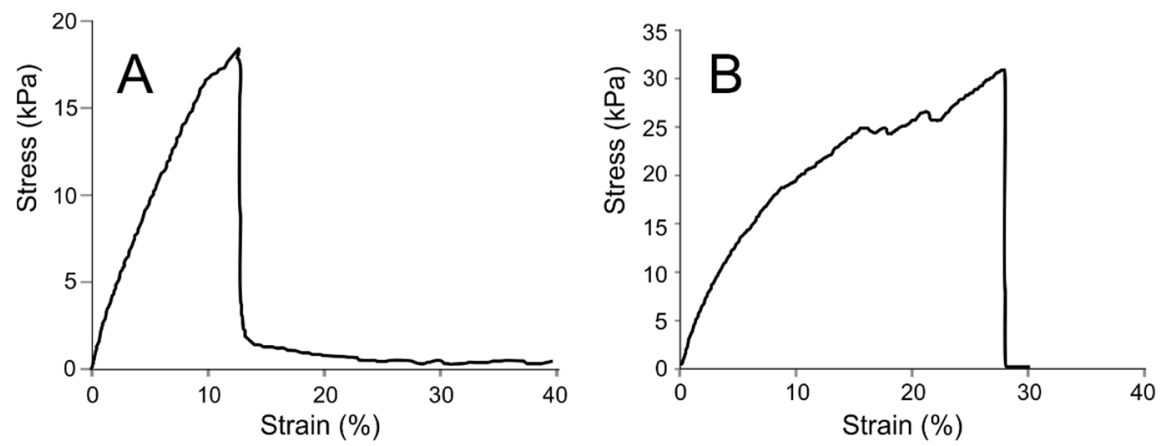

Figure 3.5 Stress-strain curves of adhesion forces to cartilage obtained from (A) a $10 \mathrm{wt}$ $\%$ Dex-TA/HA-TA (50/50) gel at a non-pretreated cartilage surface and (B) a $10 \mathrm{wt} \%$ Dex-TA/HA-TA $(50 / 50)$ gel at a pretreated surface. The cartilage surface was pretreated with a $10 \mathrm{wt} \%$ solution of Dex-TA for $5 \mathrm{~min}$.

Previously we have shown that Dex-TA gels very slowly degrade which may hamper cartilage tissue regeneration. Combining Dex-TA with HA-TA at a 50/50 weight ratio resulted in a hydrogel, which degraded in approximately 50 days [14]. It was also shown that the ultimate stress and ultimate strain, as determined by rheology even exceeds that of a Dex-TA hydrogel at a similar concentration. Based on these data we decided to test the tissue adhesive properties of this hydrogel. Both non-pretreated and pretreated cartilage surfaces (10 wt \% Dex-TA solution for $5 \mathrm{~min}$ ) were used and the results are presented in Figure 3.5. Applying a non-pretreated surface, failure was observed in $40 \%$ of cases partly in the gel and $60 \%$ of the cases at the interface. A typical stress strain curve of a successful experiment is shown in Figure 3.5A. The ultimate stress and strain measured resemble that of a Dex-TA gel. When the DexTA/HA-TA conjugates were applied on a Dex-TA pretreated surface the stress stain curve as presented in Figure 3.5B shows a more similar behavior as found for the HATA gel. In all cases, failure occurs in the gel and not at the interface and a high ultimate strength of $37 \mathrm{kPa}$ and a strain at break of $31 \%$ were found. Although a clear yield point cannot be indicated, the curve shows a clear non-linear increase in stress revealing the visco-elastic properties of the gel. The E-modulus was determined at low strain and is comparable to the Dex-TA gel. 


\subsubsection{Rheological tests}

Using oscillatory rheology we next determined the storage and loss moduli of the $10 \mathrm{wt}$ $\%$ Dex-TA, HA-TA and Dex-TA/HA-TA (50/50) enzymatically crosslinked hydrogels and the results are presented in Figure 3.6. Storage and E-moduli cannot be directly compared since the E-moduli are difficult to measure at very low strains as applied in the rheological experiments. Although the storage moduli are a factor 3 higher than the E-moduli, in general the moduli show a similar trend for the different hydrogels. Importantly, the data shows that combining Dex-TA and HA-TA in a hydrogel yields a visco-elastic injectable hydrogel with a high ultimate stress and high strain. Combined with the degradation properties such co-crosslinked hydrogels may well serve to fill defects intended for regeneration of cartilage.

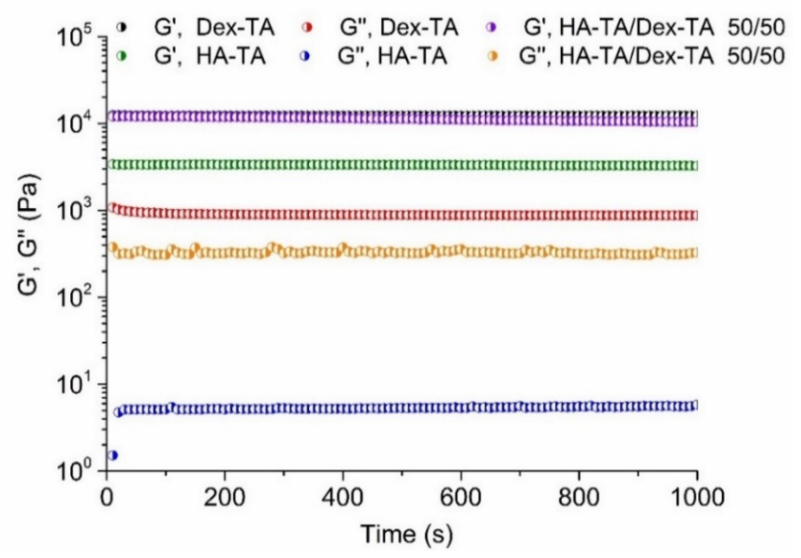

Figure 3.6 Storage and loss moduli of a 10\% Dex-TA, HA-TA and a 1:1 mixture (wt $\%$ ) of Dex-TA: HA-TA hydrogels as measured with oscillatory rheology.

\subsection{Conclusions}

In this study we have developed a method to integrate enzymatically crosslinked hydrogels to cartilage tissue and measure the adhesion properties. The results show that Dex-TA/HA-TA (50/50) hydrogels well adhere to cartilage tissue, particularly when the cartilage surface is pretreated with a polysaccharide precursor solution. The integration 
leads to failure within the gels and not at the interface of the gel and the tissue. The rheological properties of a 10\% Dex-TA/HA-TA hydrogel as well as bonding to damaged cartilage is in the order of 1 to $2 \operatorname{logs}$ better than the properties of clinically applied fibrin glue. These results are important regarding the clinical translation of hydrogels for the treatment of arthritic cartilage or cartilage defects.

\section{References}

[1] Hunziker, E. B., Articular cartilage repair: basic science and clinical progress. A review of the current status and prospects. Osteoarthritis Cartilage 2002, 10 (6), 432463.

[2] Elisseeff, J., Injectable cartilage tissue engineering. Expert Opin. Biol. Ther. 2004, 4 (12), 1849-1859.

[3] Ge, Z.; Li, C.; Heng, B. C.; Cao, G.; Yang, Z., Functional biomaterials for cartilage regeneration. J. Biomed. Mater. Res. A 2012, 100 (9), 2526-2536.

[4] Li, Y.; Rodrigues, J.; Tomas, H., Injectable and biodegradable hydrogels: gelation, biodegradation and biomedical applications. Chem. Soc. Rev. 2012, 41 (6), 2193-2221.

[5] Overstreet, D. J.; Dutta, D.; Stabenfeldt, S. E.; Vernon, B. L., Injectable hydrogels. J. Polym. Sci. B. Polym. Phys. 2012, 50 (13), 881-903.

[6] Khan, I. M.; Gilbert, S. J.; Singhrao, S. K.; Duance, V. C.; Archer, C. W., Cartilage integration: evaluation of the reasons for failure of integration during cartilage repair. Eur. Cells Mater. 2008, 16 (26-39).

[7] Spiller, K. L.; Maher, S. A.; Lowman, A. M., Hydrogels for the repair of articular cartilage defects. Tissue Eng. Part B-Rev. 2011, 17 (4), 281-299.

[8] Wang, D. A.; Varghese, S.; Sharma, B.; Strehin, I.; Fermanian, S.; Gorham, J.; Fairbrother, D. H.; Cascio, B.; Elisseeff, J. H., Multifunctional chondroitin sulphate for cartilage tissue-biomaterial integration. Nat Mater 2007, 6 (5), 385-392.

[9] Sharma, B.; Fermanian, S.; Gibson, M.; Unterman, S.; Herzka, D. A.; Cascio, B.; Coburn, J.; Hui, A. Y.; Marcus, N.; Gold, G. E.; Elisseeff, J. H., Human Cartilage 
Repair with a Photoreactive Adhesive-Hydrogel Composite. Sci. Transl. Med. 2013, 5 (167).

[10] Erickson, I. E.; Kestle, S. R.; Zellars, K. H.; Dodge, G. R.; Burdick, J. A.; Mauck, R. L., Improved cartilage repair via in vitro pre-maturation of MSC-seeded hyaluronic acid hydrogels. Biomed Mater 2012, 7 (2), 024110.

[11] Maher, S. A.; Mauck, R. L.; Rackwitz, L.; Tuan, R. S., A nanofibrous cell-seeded hydrogel promotes integration in a cartilage gap model. J. Tissue Eng. Regen. Med. 2010, 4 (1), 25-29.

[12] Balakrishnan, B.; Joshi, N.; Banerjee, R., Borate aided Schiff's base formation yields in situ gelling hydrogels for cartilage regeneration. J. Phys. Chem. B 2013, 1 (41), 5564-5577.

[13] Jin, R.; Hiemstra, C.; Zhong, Z.; Feijen, J., Enzyme-mediated fast in situ formation of hydrogels from dextran-tyramine conjugates. Biomaterials 2007, 28 (18), 27912800 .

[14] Wennink, J. W. H.; Niederer, K.; Bochynska, A. I.; Teixeira, L. S. M.; Karperien, M.; Feijen, J.; Dijkstra, P. J., Injectable Hydrogels by Enzymatic Co-Crosslinking of Dextran and Hyaluronic Acid Tyramine Conjugates. Adv. Polym. Med. 2011, 309-310 (1), 213-221.

[15] Moreira Teixeira, L. S.; Bijl, S.; Pully, V. V.; Otto, C.; Jin, R.; Feijen, J.; van Blitterswijk, C. A.; Dijkstra, P. J.; Karperien, M., Self-attaching and cell-attracting insitu forming dextran-tyramine conjugates hydrogels for arthroscopic cartilage repair. Biomaterials 2012, 33 (11), 3164-3174.

[16] Bruneel, D.; Schacht, E., Chemical modification of pullulan: 2. Chloroformate activation. Polymer 1993, 34 (12), 2633-2637. 


\section{Chapter 4}

Non-invasive near-infrared fluorescence in vivo imaging of biodegradable enzymatically crosslinked polysaccharide hydrogels*

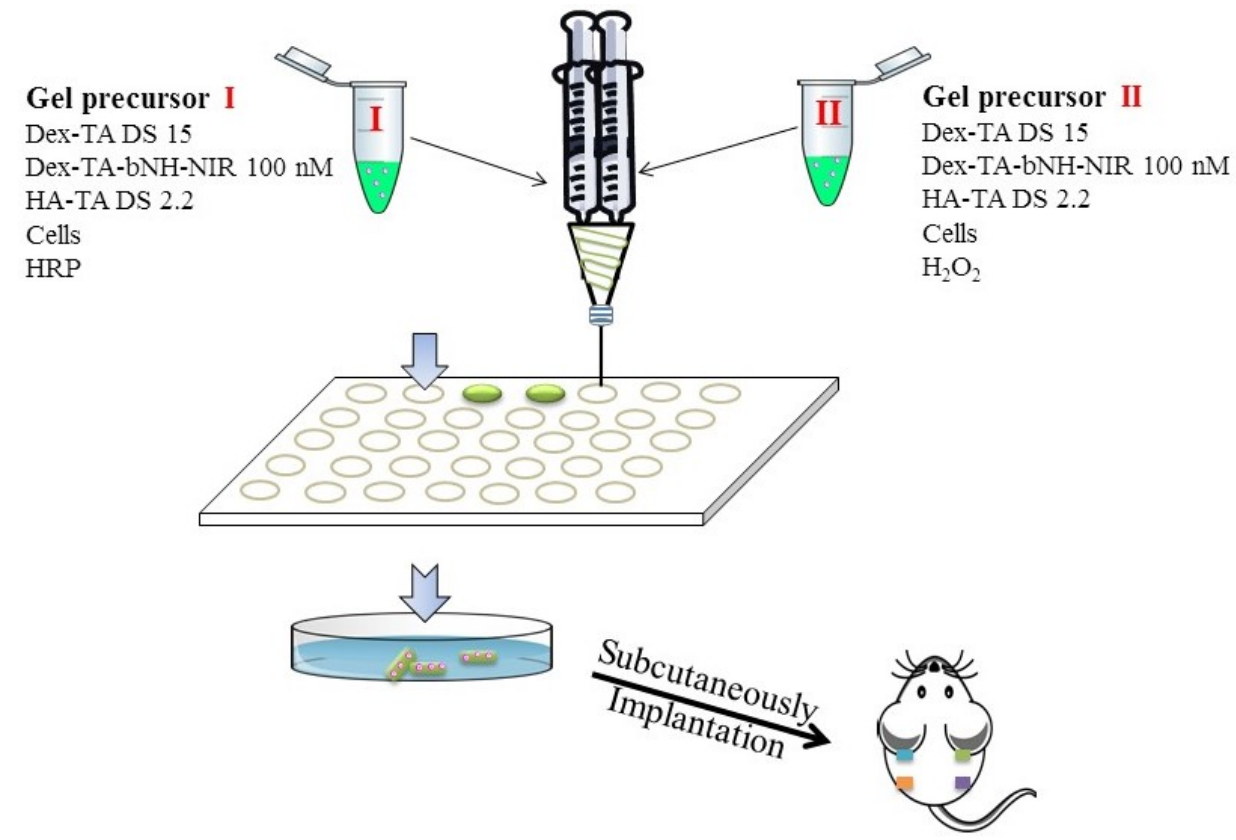

* This chapter is in preparation: Wang R., Que I., Y Fu, Both S., Chan A., Dijkstra P. J., Karperien, M, 2015. 


\section{Abstract}

An essential step in the development of in-situ forming degradable hydrogels for tissue engineering applications is the monitoring of their in vivo degradation. To avoid sacrificing large numbers of animals, in vivo real-time imaging to study the degradation of implants in time is preferred. In a long-term study over 2 months the degradation of near infrared (NIR) fluorescently labeled polysaccharide-tyramine conjugate hydrogels was followed by monitoring the decrease in NIR fluorescent intensity of subcutaneously implanted hydrogels in mice. Hydrogels implanted were prepared by the enzymatic crosslinking of dextran-tyramine or dextran-tyramine/hyaluronic acid-tyramine (50/50) conjugates. The NIR fluorescent label was covalently linked to a dextran co-conjugated with both tyramine and free amine groups. The free amine groups or NIR label had no influence on the gelation time, swelling and mechanical properties of the enzymatically crosslinked hydrogels prepared using Horseradish peroxidase in the presence of hydrogen peroxide. In vitro fluorescence imaging was performed to determine the necessary concentration for imaging prior to hydrogel implantation. Both hydrogel constructs with and without human mesenchymal stem cells/bovine primary chondrocytes in an 80/20 ratio were studied. The total fluorescence decreased to $60 \%$ for the dextran-tyramine hydrogels and to 40\% for the 50/50 dextran-tyramine hyaluronic acid-tyramine conjugates during the 10 weeks implantation period indicating hydrogel degradation. Results were similar for hydrogel/cell constructs. This research revealed that polysaccharide hydrogels can be traced by a non-invasive procedure and provides new strategies to study the degradation of injectable hydrogels in vivo. 


\subsection{Introduction}

In-situ forming hydrogels receive increasing interest in the biomedical field as materials for tissue engineering applications and in the pharmaceutical field for the controlled delivery of bioactive agents [1-3]. These materials, also referred as injectable hydrogels, may provide an environment similar to native tissue for they can retain a large amount of water. Their high permeability for nutrients and waste products allow cells incorporated to proliferate and produce a new extra cellular matrix. This process may be stimulated by the incorporation of bioactive molecules and the degradation of the hydrogel network [4-7].

Because of their substrate specificity and activity at physiological relevant conditions, an attractive concept to in-situ gelation is the enzymatic crosslinking of water soluble polymer conjugates. This research has mainly focused up to now on the use of the enzyme horseradish peroxidase. The enzyme has a high specificity for phenolic residues and in the presence of hydrogen peroxide coupling of these residues takes place at high reaction rates. Water is generated as a non-toxic by-product and the low concentration of enzyme and hydrogen peroxide necessary was shown a highly promising method to develop a new generation of injectable hydrogels. Kaplan and coworkers were the first to show the HRP mediated crosslinking of conjugates of poly(r-glutamic acid). Especially tyramine and tyrosine conjugates do rapidly form hydrogels [8]. In a similar approach, horseradish peroxidase (HRP) in combination with hydrogen peroxide $\left(\mathrm{H}_{2} \mathrm{O}_{2}\right)$ was successfully used to crosslink phenolic conjugates of polysaccharides like dextran-tyramine, chitosan-phloretic acid and hyaluronic acidtyramine conjugates [9-11]. Also for these conjugates, gelation times can be in the order of seconds, underlining the possibilities of the concept. Analogously the group of Park and coworkers studied tyramine conjugates of branched poly(propylene oxide)-bpoly(ethylene oxide) and gelatin. The physico-chemical and biological properties of the hydrogels could be tuned according various parameters, e.g. HRP and $\mathrm{H}_{2} \mathrm{O}_{2}$ concentration, peptide conjugation and phenolic content [12-15]. Recent research from Lakshmi et al. showed that in situ forming phenolic-conjugated chitosan hydrogels can be used as carriers for the sustained release of rhBMP-2. In vivo animal studies revealed that rhBMP-2 remained bioactive and stimulated a more rapid closure of a defect within 
4-8 weeks post implantation [16]. The in vitro degradation of hydrogels based on synthetic or natural polymers in relation to tissue regeneration or the release of bioactive agents has been widely studied [17]. Despite the increasing number of studies to degradable hydrogels, either by physical or chemical crosslinking, in vivo degradation studies are hampered by the large number of animals required to acquire statistically relevant data. Moreover, only qualitative insight in the degradation and tissue regeneration process is obtained.

Non-invasive imaging technologies become increasingly important in studies towards tissue-engineered products. These technologies do not provide a single solution to tissue regeneration processes but open up strategies to ex vivo monitoring in time. A commonly used technique for tracking hydrogel degradation is fluorescence spectroscopy. Artzi and co-workers demonstrated a noninvasive assessment of implanted PEG/dextran crosslinked hydrogels sequentially to model hydrolytical and enzymatical degradation of materials using non-invasive fluorescence imaging [18]. Recently, Wang and co-workers showed that in vivo fluorescent tracking of physically crosslinked synthetic amphiphilic copolymer hydrogels (poly(ethylene glycol)-b-poly( $\varepsilon$ caprolactone)-b- poly(ethylene glycol)) over a period of 5 weeks was possible by covalently conjugating Rhodamine B [19]. More recently it was shown that the use of near infrared (NIR) labels may provide insight in the degradation of implanted hydrogels. A NIR fluorophore absorbs light between $\sim 650 \mathrm{~nm}$ and $950 \mathrm{~nm}$, where biological tissue components do not absorb light significantly, allowing accurate imaging at tissue depths ranging from 1 to $4 \mathrm{~cm}$ [20]. Moreover, NIR fluorophores are stable at physiological conditions, and most importantly, the use of fluorescent labels enables an extensive long-term in vivo investigation of the degradation of the hydrogels by imaging without the need to sacrifice large numbers of animals. In a recent report from Kim and co-workers, the degradation of a crosslinked collagen scaffold conjugated with the zwitterionic NIR fluorophore (ZW800-1) was monitored in vivo up to 4 weeks and results correlated well with in vitro degradation profiles using collagenase [21]. In another study from Jalani and co-workers, upconverted silica coated lanthanide-doped nanoparticles were used to follow the degradation of physically crosslinked chitosanhyaluronic acid thermosensitive hydrogels in explanted intervertebral discs up to 3 weeks [22]. In vitro and ex-vivo release profiles according to NIR imaging afforded 
different results, which were mathematically correlated. Up to now no studies on biodegradable enzymatically crosslinked hydrogels with or without cells incorporated using non-invasive real-time monitoring have been reported.

We here describe polysaccharide based injectable hydrogel systems co-conjugated with a near-infrared fluorescent (NIR) label. To this objective, a dextran conjugated with both tyramine and free amine groups was labeled with the near-infrared dye, IRDye $800 \mathrm{CW}$. The effects of introducing free amine groups on the solution and gel properties and NIR dye stability upon enzymatic crosslinking were studied. Finally, bovine articular chondrocytes and human mesenchymal stem cells were encapsulated in the NIR-labeled hydrogels to assess their biological performance and effect on the hydrogel degradation.

\subsection{Materials and methods}

\subsubsection{Materials}

Dextran (Dex) ( $\mathrm{M}_{\mathrm{w}} 15$ to $\left.25 \mathrm{~kg} / \mathrm{mol}-\mathrm{M}_{\mathrm{n}} 16 \mathrm{~kg} / \mathrm{mol}\right)$ was obtained from Sigma-Aldrich (Miami, USA) and dried by lyophilization. Sodium hyaluronate $(\mathrm{Mw}=15-30 \mathrm{kDa}$, Pharmaceutical grade) was purchased from Contipro (Dolni Dobrouc, Czech Republic) and used without further purification. 4-Nitrophenyl chloroformate (PNC) (96\%) was purchased from Sigma-Aldrich and was sublimated before use. Lithium chloride ( $\mathrm{LiCl})$ (99\%) was obtained from Sigma-Aldrich and dried at $110{ }^{\circ} \mathrm{C}$ in an oven before use. Tyramine (TA) (99\%), anhydrous pyridine (99.8\%), anhydrous N,Ndimethylformamide (DMF) (99.8\%), trifluoroacetic acid (TFA), sodium hydroxide ( $\mathrm{NaOH}), \mathrm{N}$-(3-dimethylaminopropyl)-N'-ethylcarbodiimide hydrochloride (EDAC), $\mathrm{N}$ hydroxysuccinimide (NHS), hydrogen peroxide $\left(\mathrm{H}_{2} \mathrm{O}_{2}\right)$, horseradish peroxidase (HRP, type VI, 261 purpurogallin unit/mg solid) and $\mathrm{N}-\mathrm{Boc}-1,4-$ butanediamine $\left(\mathrm{NH}_{2}\right.$-Boc) were obtained from Sigma-Aldrich and used without further purification. IR Dye ${ }^{\circledR}$ infrared dye $800 \mathrm{CW}$ NHS ester was obtained from LI-COR Biosciences (Lincoln, NE). Phosphate buffered saline (PBS, $150 \mathrm{mM}, \mathrm{pH}$ 7.4) was purchased from Gibco Life Technologies Ltd (Paisley, UK). All other solvents were purchased from Biosolve (Valkenswaard, The Netherlands) and used as received. 


\subsubsection{Synthesis}

Dextran was reacted with p-nitrophenyl chloroformate (PNC) to form p-nitrophenyl carbonate conjugates, which were then treated with primary amine-containing compounds [9].

Dextran-tyramine (Dex-TA): In a typical experiment, dextran $(3.0 \mathrm{~g}, 56.3 \mathrm{mmol} \mathrm{OH}$ groups) was dissolved in DMF $(200 \mathrm{~mL}$, containing $2.4 \mathrm{~g}$ of $\mathrm{LiCl})$ at $90{ }^{\circ} \mathrm{C}$ under a nitrogen atmosphere. After the dextran was dissolved, the mixture was cooled and thermostated at $0{ }^{\circ} \mathrm{C}$. Pyridine $(1.5 \mathrm{~mL}, 18.6 \mathrm{mmol})$ and PNC $(2.6 \mathrm{~g}, 12.9 \mathrm{mmol})$ were added to the solution while stirring. The reaction was allowed to proceed for one hour and the product was precipitated in cold ethanol. The precipitate was filtered and washed with ethanol and diethyl ether, and then dried in a vacuum oven. The degree of activation (DA), defined as the number of activated groups per 100 anhydroglucose rings, was determined by ${ }^{1} \mathrm{H}-\mathrm{NMR}$ : Dex-PNC 26 . Yield: $4.24 \mathrm{~g}$ (PNC conversion 42.1\%). ${ }^{1} \mathrm{H}-\mathrm{NMR}\left(\mathrm{DMSO}_{\mathrm{d}}\right.$ ): $\delta$ 3.0-4.0 (m, dextran glucosidic protons), 4.2-5.8 (m, dextran anomeric protons and $\mathrm{OH}$ protons), 7.56, $8.32 \mathrm{ppm}$ (d, aromatic protons).

Next, Dex-PNC 26 (4.24 g, 5.43 mmol PNC groups) was dissolved in $50 \mathrm{~mL}$ of DMF. Tyramine $(0.89 \mathrm{~g}, 6.52 \mathrm{mmol})$ was added under a nitrogen flow and the reaction was allowed to proceed for one hour. The product was precipitated in cold ethanol, filtered and washed with ethanol and diethyl ether. The tyramine conjugated dextran was further purified by dialysis (MWCO $1000 \mathrm{Da}$ ) against a $150 \mathrm{mM} \mathrm{NaCl}$ solution for $48 \mathrm{~h}$ and deionized water for $24 \mathrm{~h}$ and isolated by lyophilization. The degree of substitution (DS), defined as the number of substituted groups per 100 anhydroglucose rings, was determined by ${ }^{1} \mathrm{H}-\mathrm{NMR}$ : Dex-TA 15 . Yield: $3.94 \mathrm{~g}$ (99.6\%). ${ }^{1} \mathrm{H}-\mathrm{NMR}$ (DMSO-d ${ }_{6}$ ): $\delta 3.0-$ 4.0 ( $\mathrm{m}$, dextran glucosidic protons), 4.2-5.5 ( $\mathrm{m}$, dextran anomeric protons and $\mathrm{OH}$ protons), 6.66, 6.98 (d, aromatic protons).

Dextran-butylamine (Dex-bNH $\left.\mathbf{H}_{2}\right)$ : Typically, Dex-PNC 23 (0.5 g, 0.58 mmol PNC groups) was dissolved in $8 \mathrm{~mL}$ of DMF. N-Boc-1,4-butanediamine $(0,164 \mathrm{~g}, 0,87 \mathrm{mmol})$ was added under a nitrogen flow and the reaction was allowed to proceed for one hour at room temperature. The product was precipitated in cold ethanol, filtered and washed with ethanol and diethyl ether, and then dried in a vacuum oven. The degree of substitution, defined as the number of substituted groups per 100 anhydroglucose rings, 
was determined by ${ }^{1} \mathrm{H}-\mathrm{NMR}$ : Dex-(bNHBoc) $)_{12}$, Yield: $0.35 \mathrm{~g}(60.1 \%)$. ${ }^{1} \mathrm{H}-\mathrm{NMR}$ $\left(\right.$ DMSO-d $\left.\mathrm{d}_{6}\right): \delta$ 1.2-1.5 (m, methyl protons and $-\mathrm{CH}_{2}-\left(\mathrm{CH}_{2}\right)_{2}-\mathrm{CH}_{2} \mathrm{NHBoc}$ protons), 3.04.2 (m, dextran glucosidic protons), 4.4-5.8 (m, dextran anomeric protons and $\mathrm{OH}$ protons).

In the next step, Dex-(bNHBoc $)_{12}(0.35 \mathrm{~g}, 0.19 \mathrm{mmol})$ was dissolved in $8.5 \mathrm{~mL}$ of deionized water. Trifluoroacetic acid (TFA) $(0.35 \mathrm{~mL}, 4.57 \mathrm{mmol})$ was added under a nitrogen flow and stirred overnight. The solution was neutralized to $\mathrm{pH} 7$ by a $2 \mathrm{M}$ $\mathrm{NaOH}$ solution and the product was purified by dialysis (MWCO $1000 \mathrm{Da}$ ) against deionized water for 48 hours and isolated by lyophilisation. The degree of substitution, defined as the number of substituted groups per 100 anhydroglucose rings, was determined by ${ }^{1} \mathrm{H}-\mathrm{NMR}$ : Dex-(bNH$)_{12}$, Yield: $0.16 \mathrm{~g}$ (36.6\%). ${ }^{1} \mathrm{H}-\mathrm{NMR}\left(\mathrm{DMSO}_{\mathrm{d}}\right)$ ): $\delta$ 1.4-1.5 (b, - $\mathrm{CH}_{2}-\left(\mathrm{CH}_{2}\right)_{2}-\mathrm{CH}_{2} \mathrm{NH}_{2}$ protons), 3.0-4.2 (m, dextran glucosidic protons), 4.45.8 ( $\mathrm{m}$, dextran anomeric protons and $\mathrm{OH}$ protons).

Dextran-tyramine-butylamine (Dex-TA-bNH $\mathbf{H}_{2}$ ): Dex-PNC was first reacted with $N$ Boc-1,4-diaminobutane and then with tyramine. The protecting $t$-butyloxycarbonyl group was removed by reaction with TFA. In a typical experiment, Dex-PNC $26(1.0 \mathrm{~g}$, $1.28 \mathrm{mmol}$ PNC groups) was dissolved in $16 \mathrm{~mL}$ of DMF. $N$-Boc-1,4-diaminobutane $(0.09 \mathrm{~g}, 0.49 \mathrm{mmol})$ was added under a nitrogen flow and the reaction was allowed to proceed for $15 \mathrm{~min}$. Thereafter tyramine $(0.20 \mathrm{~g}, 1.48 \mathrm{mmol})$ was added and the reaction was allowed to proceed for one hour. The product was precipitated in cold ethanol, the precipitate was filtered and washed with ethanol and diethyl ether, and then dried in a vacuum oven. The degree of conjugated tyramine and butylamine groups, defined as the number of substituted groups per 100 anhydroglucose rings, was determined by ${ }^{1} \mathrm{H}-$ NMR: Dex-TA $10-\left(\right.$ bNHBoc) 6 , Yield: 0.85 g (77.6 \%). ${ }^{1} \mathrm{H}-\mathrm{NMR}$ (DMSO-d6): $\delta$ 1.2-1.5 ppm (m, methyl protons and $-\mathrm{CH}_{2}-\left(\mathrm{CH}_{2}\right)_{2}-\mathrm{CH}_{2} \mathrm{NHBoc}$ protons), 3.0-4.0 (m, dextran glucosidic protons), 4.2-5.8 (m, dextran anomeric protons and $\mathrm{OH}$ protons), 6.66, 6.98 (d, aromatic protons).

In the second step, Dex-TA $10-(\mathrm{bNHBoc})_{6}(0.85 \mathrm{~g}, 0.47 \mathrm{mmol})$ was dissolved in 10 $\mathrm{mL}$ of deionized water. Trifluoroacetic acid (TFA) $(1 \mathrm{~mL}, 13.06 \mathrm{mmol})$ was added under a nitrogen atmosphere and stirred overnight. The reaction mixture was neutralized by a $2 \mathrm{M} \mathrm{NaOH}$ solution and purified by dialysis (MWCO $1000 \mathrm{Da}$ ) against a $150 \mathrm{mM}$ $\mathrm{NaCl}$ solution for 48 hours and deionized water for 24 hours and then isolated by 
lyophilization. The degree of substitution, defined as the number of substituted groups per 100 anhydroglucose rings, was determined by ${ }^{1} \mathrm{H}-\mathrm{NMR}$ : Dex-TA $\mathrm{T}_{10}-\left(\mathrm{bNH}_{2}\right)_{6} \cdot{ }^{1} \mathrm{H}-$ NMR (DMSO-d6): $\delta$ 1.4-1.5 ppm (m, $-\mathrm{CH}_{2}-\left(\mathrm{CH}_{2}\right)_{2}-\mathrm{CH}_{2} \mathrm{NH}_{2}$ protons), 3.0-4.0 (m, dextran glucosidic protons), 4.2-5.8 ( $\mathrm{m}$, dextran anomeric protons and $\mathrm{OH}$ protons), 6.66, 6.98 (d, aromatic protons).

IRDye ${ }^{\circledR}$ 800CW labeled dextran: The IRdye ${ }^{\circledR} 800 \mathrm{CW}$ NHS ester $(10 \mu \mathrm{L}, 1 \mathrm{mM}$ in PBS) was added to $1 \mathrm{~mL}$ of PBS buffer ( $\mathrm{pH}$ 8.2) and the resulting solution was subsequently added to $9 \mathrm{~mL}$ of a Dex-TA $10^{-}\left(\mathrm{bNH}_{2}\right)\left(10 \mathrm{mg}, 3.3 \times 10^{-3} \mathrm{mmol} \mathrm{NH}_{2}\right)$ solution in PBS. The reaction was allowed to proceed overnight at room temperature in the dark. The product was purified by dialysis (MWCO $1000 \mathrm{Da}$ ) against deionized water for 48 hours and isolated by lyophilization.

Hyaluronic acid-tyramine conjugate (HA-TA): Typically, sodium hyaluronate (1.0 g) was dissolved in $20 \mathrm{~mL}$ of MES buffer (0.1 M, pH 6.0). EDAC (240 mg, $1.25 \mathrm{mmol})$ and NHS (144 mg, $1.25 \mathrm{mmol}$ ) were separately dissolved in $5.0 \mathrm{~mL}$ of MES buffer. The solutions were mixed and after $30 \mathrm{~min}$ of stirring a solution of tyramine (342 $\mathrm{mg}, 2.50$ $\mathrm{mmol}$ ) in $6 \mathrm{~mL}$ of DMF was added and the resulting solution was stirred for 3 days. The mixture was neutralized with $1 \mathrm{M} \mathrm{NaOH}$ and dialyzed (MWCO 1000), first with PBS and then MilliQ-water. The HA-TA conjugate was obtained after lyophilization as a white foam. Yield: $0.72 \mathrm{~g}(71 \%)$. The degree of substitution, defined as the number of substituted groups per 100 saccharide rings, was determined by ${ }^{1} \mathrm{H}-\mathrm{NMR}$ : HA-TA 2.2 . ${ }^{1} \mathrm{H}-\mathrm{NMR}\left(\mathrm{D}_{2} \mathrm{O}\right): \delta 1,9$ (s, methyl protons), 2,4-2,8 (m, glucosidic protons), 3.2-4.1 (m, glucosidic protons), 4.2-4,6 (d, 1H, glucosidic protons), 6.86 and 7.17 (d, aromatic protons).

Chondrocytes (bCh) and human mesenchymal stem cells (hMsc): Bovine primary chondrocytes (bCh) were isolated as previously reported [23] and cultured in chondrocyte expansion medium (DMEM with 10\% heat inactivated fetal bovine serum, $1 \%$ penicillin/streptomycin (Gibco), $0.5 \mathrm{mg} / \mathrm{mL}$ fungizone (Gibco), $0.01 \mathrm{M}$ MEM nonessential amino acids (Gibco) and $0.04 \mathrm{mM} \mathrm{L-proline)} \mathrm{at} 37{ }^{\circ} \mathrm{C}$ in a humidified atmosphere $\left(95 \%\right.$ air $\left./ 5 \% \mathrm{CO}_{2}\right)$. Human bone marrow derived mesenchymal stem cells (hMsc) were isolated as previously reported [24] and cultured in MSC proliferation medium ( $\alpha$-MEM (Gibco) supplemented with $10 \%$ fetal bovine serum, $1 \%$ L-glutamin (Gibco), $0.2 \mathrm{mM}$ ascorbic acid (Gibco), $100 \mathrm{U} / \mathrm{mL}$ penicillin, $10 \mu \mathrm{g} / \mathrm{mL}$ streptpmycin 
and $1 \mathrm{ng} / \mathrm{mL} \mathrm{bFGF}$ ). The use of human material was approved by a local medical ethical committee. All reagents used were purchased from Invitrogen (Paisley, UK) unless otherwise stated.

Hydrogel-cells constructs: Hydrogels without cells or containing hMsc/bCh (80/20) were prepared under sterile conditions by mixing gel solutions and a cell suspension with a freshly prepared mixture of $\mathrm{HRP}$ and $\mathrm{H}_{2} \mathrm{O}_{2}$. Gel precursor solutions were made using chondrocyte differentiation medium and $\mathrm{HRP}$ and $\mathrm{H}_{2} \mathrm{O}_{2}$ stock solutions were made using PBS. Typically, $50 \mu \mathrm{L}$ of a Dex-TA $15 / \mathrm{HA}-\mathrm{TA}_{2.2}(50 / 50 \mathrm{wt} / \mathrm{wt})$ polymer solution $(12.5 \mathrm{wt} \%)$ and $12.5 \mu \mathrm{L}$ of a HRP stock solution $(150 \mathrm{U} / \mathrm{mL})$ were added to a hMsc/bCh $(80 / 20)$ pellet $\left(6.25 \times 10^{5}\right.$ cells $)$, cells were resuspended by gently mixing and the suspension was added to one chamber of a double syringe (MixPac ${ }^{\mathrm{TM}}$, Switzerland). Similarly, $50 \mu \mathrm{L}$ of Dex-TA $15 / \mathrm{HA}-\mathrm{TA}_{2.2}(50 / 50)$ polymer solution $(12.5 \mathrm{wt} \%)$ and 12.5 $\mu \mathrm{L}$ of a $\mathrm{H}_{2} \mathrm{O}_{2}$ stock solution $(0.3 \mathrm{wt} \%$ ) were mixed with an hMsc/bCh (80/20) pellet $\left(6.25 \times 10^{5}\right.$ cells $)$ and added to the other chamber of the double syringe. Hydrogels $(100$ $\mu \mathrm{L}, 10 \mathrm{wt} \%$ ) were prepared by injecting the solutions into a home-made Teflon model plate containing 35 wells (100 $\mu \mathrm{L} /$ well, sterilized before use). The final cell seeding density in the gel was $10 \times 10^{6} / \mathrm{mL}$. Gels were removed from the mold and put into chondrocyte proliferation medium and cultured overnight before implantation.

In vivo Implantation: Balb c/nude male mice, 6-8 weeks of age were obtained from Charles River Laboratory (Lentilly,France ) and kept in standard cages with access to food and water ad libitum. All was conducted with approval of the Animal Ethic Committee of Leiden University Medical Center. Labeled gels $(100 \mu \mathrm{L}, 100 \mathrm{nM}$ NIR dye, $10 \mathrm{wt} \%$ ) of Dex-TA 15 , Dex-TA $15 / \mathrm{HA}-\mathrm{TA}_{2.2}$ 50/50 with or without $\mathrm{hMsc} / \mathrm{bCh}$ (80/20) were first washed with PBS and were subcutaneously implanted under $2 \%$ of Isoflurane anesthesia in four different pockets. The location of the four conditions were randomly chosen $(n=6)$.

\subsubsection{Characterization}

${ }^{1}$ H-NMR (400 MHz): Spectra were recorded using a Bruker AVANCE III $400 \mathrm{MHz}$ (Nanobay) spectrometer. The signals of solvent residues were used as reference for the 
${ }^{1} \mathrm{H}$ NMR chemical shift and were set at $\delta 2.50$ for DMSO-d6 and at $\delta 4.79$ for $\mathrm{D}_{2} \mathrm{O}$. Polymers were dissolved at a concentration of $0.010 \mathrm{~g} / \mathrm{mL}$.

Dynamic Light Scattering (DLS): Size distributions were measured with DLS using a Malvern Instruments Zetasizer Nano ZS at $25^{\circ} \mathrm{C}$, equilibration time was set to 2 min and a $173^{\circ}$ backscatter angle was used. All measurements were recorded using polystyrene capillary folded cells type 1070. Aqueous solutions of the polymers were prepared at a concentration of $3 \mathrm{mg} / \mathrm{mL}$ by dissolving in MilliQ water overnight. The solution was filtered $(0.22 \mu \mathrm{m})$ prior to measurements, data were obtained from at least three measurements.

Hydrogel formation and gelation time: Hydrogel samples $(\sim 0.25 \mathrm{~mL})$ at a final polymer concentration of $10 \mathrm{wt} \%$ were prepared in vials at room temperature. In a typical procedure, a polymer solution $(100 \mu \mathrm{L}, 12.5 \mathrm{wt} \%$ in PBS) and a freshly prepared solution of $\mathrm{H}_{2} \mathrm{O}_{2}(25 \mu \mathrm{L}, 0.3 \mathrm{wt} \%$ stock solution in PBS) were mixed well. Correspondingly, a polymer solution $(100 \mu \mathrm{L}, 12.5 \mathrm{wt} \%$ in PBS $)$ and a freshly prepared solution of HRP ( $25 \mu \mathrm{L}, 150 \mathrm{U} / \mathrm{mL}$ stock solution in PBS) were mixed thoroughly. The two solutions were added together, mixed gently by vortex and the time to form a gel (denoted as gelation time) was determined by using the vial tilting method. The experiment was performed in triplicate.

Rheological analysis: Rheological experiments were carried out with a MCR 301 rheometer (Anton-Paar) using parallel plates (ø $25 \mathrm{~mm}, 0^{\circ}$ ) set to $37^{\circ} \mathrm{C}$ in the oscillatory mode. In a typical experiment, preformed gel samples $(\sim 0.25 \mathrm{~mL}, 10 \mathrm{wt} \%)$ were placed at the center of the ground plate, while the upper plate was lowered to a gap size of 0.3 $\mathrm{mm}$. A layer of oil was introduced around the gel sample to prevent water evaporation. The storage $\left(G^{\prime}\right)$ and loss $\left(G^{\prime}\right.$ ') modulus were recorded under oscillatory shear. Experiments were performed using a strain $(\gamma)$ of $1 \%$ and frequency $(\omega)$ of $1 \mathrm{~Hz}$.

Degree of swelling: For the swelling tests, prepared gels $(\sim 0.25 \mathrm{~mL})$ were lyophilized $\left(\mathrm{W}_{\mathrm{d}}\right)$. Subsequently, $3 \mathrm{~mL}$ of fresh PBS solutions were applied to the dried hydrogels. The gels were incubated at $37{ }^{\circ} \mathrm{C}$ and allowed to reach swelling equilibrium (approximately 2.5 days). The excess of PBS was removed and samples were weighed $\left(\mathrm{W}_{\mathrm{s}}\right)$. Data were obtained from at least three independent measurements and the swelling ratio was expressed as: $S R=\frac{W_{S}-W_{d}}{W_{d}}$. 
Fluorescence imaging: In order to determine the emission of the NIR dye conjugated to Dex-TA-bNH ${ }_{2}$, intensity measurements were performed using an Odyssey® Infrared Imaging System (LI-COR; Lincoln, NE), version 1.0.4 (ODY 2468), as instructed in the application protocol manual. The fluorescence signals were measured at $800 \mathrm{~nm}$. The images were analyzed using the Odyssey Imaging Studio ${ }^{\mathrm{TM}}$ Software with In-Cell Western $^{\mathrm{TM}}$ Analysis Module, which automatically finds wells for 96-well microplates and performs radiometric calculations using the $800 \mathrm{~nm}$ channel of the Odyssey Imager. The fluorescent signal from each well was quantified by the integrated intensities and numerical data were analyzed using GraphPad Prism 6.0.

In vivo imaging: A PEARL impulse imaging camera (LI-COR; Lincoln, NE) was used for the in vivo imaging. Mice were imaged under 1.5\% Isoflurane anesthesia in ventral and dorsal sites at pre- and post-implantation as well as at $1 \mathrm{~h}$ and 1, 3, 7, 14, 28, 41, 53, 63 and $70 \mathrm{~d}$. The results were analyzed with Pearl impulse software 3.01. Mice were sacrificed with $\mathrm{CO}_{2}$ at day 70 .

\subsection{Results and discussion}

\subsubsection{Synthesis}

Dextran conjugates, as depicted in Scheme 4.1, were prepared by firstly activating the hydroxyl groups of dextran $(\mathrm{Mw}=16 \mathrm{KDa})$ with p-nitrophenyl chloroformate $(\mathrm{PNC})$ to give p-nitrophenyl carbonate conjugates (Dex-PNC). The degree of activation (DA), defined as the number of conjugated moieties per 100 dextran anhydroglucose rings, was determined using ${ }^{1} \mathrm{H}$ NMR by comparing the integrals of signals at $\delta$ 4.2-5.6 (anomeric and $\mathrm{OH}$ protons) and $\delta$ 7.4-8.5 (aromatic protons) (Figure 4.1 A). Dextran tyramine conjugates were prepared by reacting the Dex-PNC with an excess of tyramine. Dex-TA conjugates with DS values of 10 and 15 could be prepared by changing the degree of activation using different molar feed ratio of p-nitrophenyl chloroformate to dextran hydroxyl groups prior to reaction with the amine (Figure 4.1 B). 


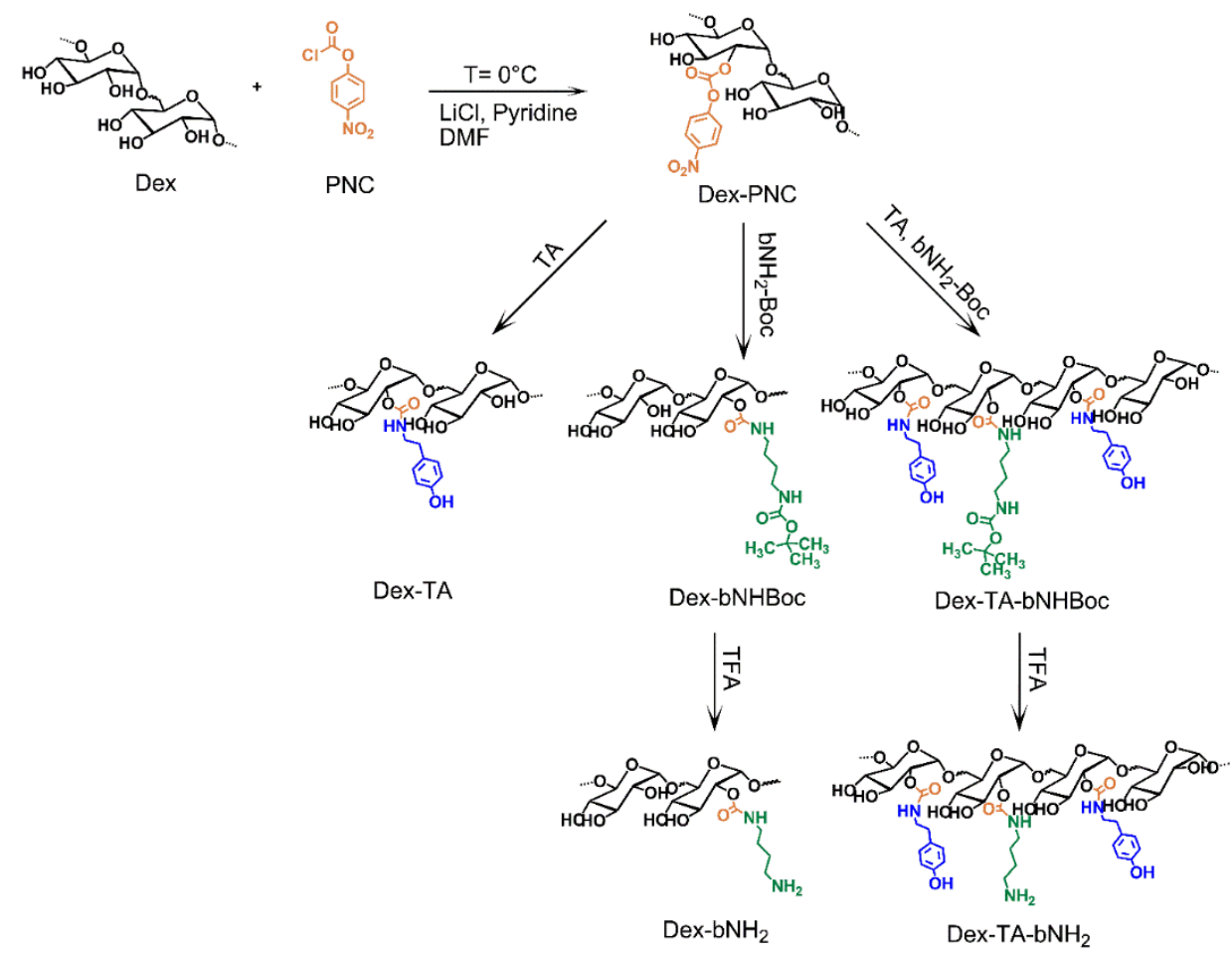

Scheme 4.1 Synthesis of Dex-TA, Dex-bNH 2 and Dex-TA-bNH 2 . (Dex: Dextran; PNC: 4-nitrophenyl chloroformate; TA: tyramine; $\mathrm{bNH}_{2}$-Boc: N-Boc-1,4-butanediamine; TFA: trifluoroacetic acid).

In a similar way dextran was conjugated with butyl-amine groups by first reacting the Dex-PNC with the mono Boc-protected 1,4-butanediamine. The Boc-group was removed upon reaction with trifluoroacetic acid. The successful conjugation was confirmed by ${ }^{1} \mathrm{H}-\mathrm{NMR}$ showing the butylamine central methylene protons at $\delta 1.52$ (Figure 4.1 C). Conjugation of dextran with both tyramine and aminobutyl groups was preferably done by first addition of N-Boc-1,4-diaminobutane and after $15 \mathrm{~min}$. of reaction addition of tyramine. After deprotection of the Boc-groups the DS of the two different groups was determined by ${ }^{1} \mathrm{H}$ NMR (Figure 4.1 D). By changing the molar feed ratios of the N-Boc-1,4-diaminobutane and tyramine, dextran conjugates with different DS values of free amine and tyramine groups were obtained after deprotection. 


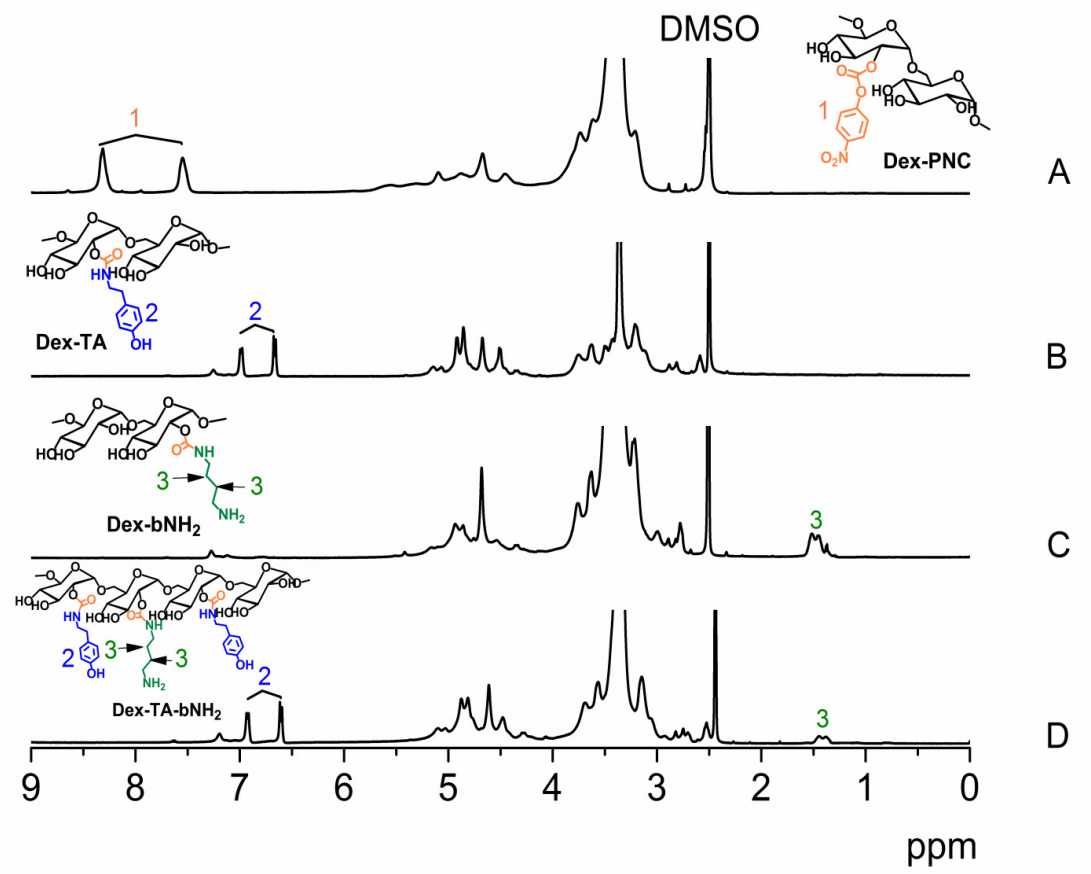

Figure 4.1 ${ }^{1} \mathrm{H}-\mathrm{NMR}$ spectra $\left(\mathrm{DMSO}-\mathrm{d}^{6}\right)$ of Dex-PNC (A), Dex-TA 10 (B), Dex- $\left(\mathrm{bNH}_{2}\right)_{12}$ (C) and Dex- $\mathrm{TA}_{10}-\left(\mathrm{bNH}_{2}\right)_{6}(\mathrm{D})$.

\subsubsection{Solution properties}

Polymer-solvent interactions generally dictate the conformation of a dissolved polymer. It is well known that water is a theta solvent for dextran [25]. At high dilution $(0.3 \mathrm{wt} \%)$ dextrans are present as small aggregates in water or PBS and the formation of these aggregates was explained by the hydrophobic interactions between the anhydroglucose rings and hydrogen bonding between $\mathrm{OH}$ groups [26]. Upon conjugation of dextran with hydrophobic tyramine groups and/or hydrophilic free amine groups (Dex-TA, Dex$\mathrm{bNH}_{2}$ and Dex-TA-bNH${ }_{2}$ ) large differences were observed in the average aggregate diameters at high dilution as measured using dynamic light scattering (DLS). In Figure 4.2A the distribution of the aggregates is presented in an intensity plot. A bimodal distribution was observed with larger aggregates present. It has to be emphasized that the intensity of the light scattered by the larger aggregates is much larger than that of the smaller particles (power 6) and the number plot as depicted in Figure 4.2B will give a 
better illustration of particles most present. From the intensity plots an average aggregate size distribution of the different conjugates were obtained and the results are presented in Table 4.1. Dex-TA conjugates with a DS of $10(46.2 \pm 8.8)$ and $15(37.1 \pm$ 7.9) $\mathrm{nm}$ were considerably smaller than that of dextran $(158 \pm 17 \mathrm{~nm})$. This decrease can be explained by the hydrophobic interactions due to the conjugation with tyramine groups. The average size of dextran conjugated with butylamine groups (DS 12) was approximately $3.6 \pm 1.2 \mathrm{~nm}$, demonstrating an almost complete disappearance of aggregation. The free amine group containing dextran conjugates appear to form monomolecular micelles in water or PBS and are stabilized by the protonated amine groups (pKa of approximately 10.5) likely located at the surface of the aggregates. When dextran was conjugated with both tyramine and butylamine groups (DS $10: 6$ and $15: 6$ ), intermediate average aggregate sizes of $12.5 \pm 0.6 \mathrm{~nm}$ and $11.9 \pm 1.5 \mathrm{~nm}$, respectively, were found, values also significantly smaller than the aggregate size of Dex-TA conjugates.

Table 4.1 Composition, dynamic size (intensity), zeta potential and polydispersity (PDI) of dextran conjugates.

\begin{tabular}{c|c|c|c|c}
\hline \hline Entry & DS $^{a}$ & $\begin{array}{c}\text { Size average } \\
(\mathrm{nm})\end{array}$ & $\begin{array}{c}\text { Zeta potential } \\
(\mathrm{mV})\end{array}$ & PDI \\
\hline Dex & - & $158 \pm 17$ & $-5.8 \pm 2.3$ & 0.58 \\
\hline Dex-TA & 10 & $46 \pm 9$ & $-18.0 \pm 3.2$ & 0.59 \\
\hline Dex-TA & 15 & $37 \pm 8$ & $-19.8 \pm 5.1$ & 0.36 \\
\hline Dex-bNH & 12 & $3.6 \pm 1.2$ & $15.3 \pm 4.2$ & 0.32 \\
\hline Dex-TA-bNH & $10: 6$ & $12.5 \pm 0.6$ & $3.2 \pm 1.4$ & 0.49 \\
\hline Dex-TA-bNH & $15: 6$ & $11.9 \pm 1.5$ & $5.8 \pm 2.0$ & 0.40 \\
\hline HA-TA & 2.2 & $11.1 \pm 1.6$ & $-3.0 \pm 0.6$ & 0.54 \\
\hline \hline
\end{tabular}

${ }^{a}$ : DS values were determined from ${ }^{1} \mathrm{H}$ NMR spectra. 
A

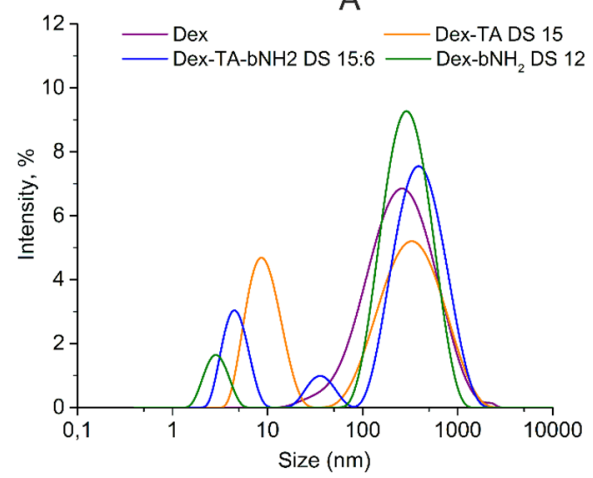

B

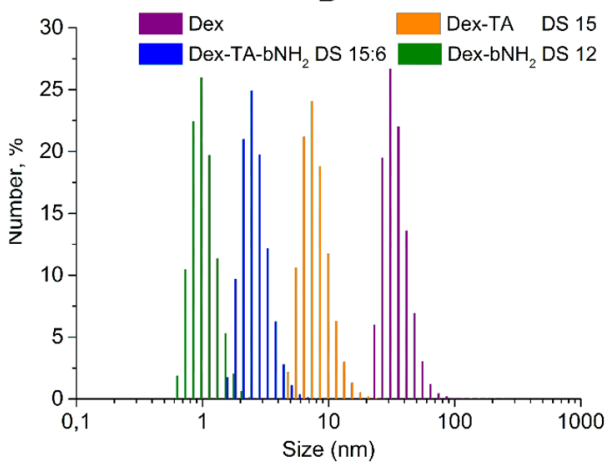

Figure 4.2 Size distribution profiles of dextran and dextran conjugates at a concentration of $0.3 \mathrm{wt} \%$ in MiliQ water as determined by DLS, (A) Intensity plot, (B) Number plot.

The large changes in the aggregation behavior indicated a change in surface charge of the aggregates. The zeta potentials of the aggregates revealed that upon conjugation with tyramine the zeta potential as determined for dextran $(-5.8 \mathrm{mV})$ changed to -18.0 $\mathrm{mV}$ for Dex-TA 10 and to $-19.8 \mathrm{mV}$ for Dex-TA $\mathrm{T}_{15}$. However, conjugation with aminobutyl groups led in all cases to positively charged type aggregates, with a highest value of $15.3 \mathrm{mV}$ for the Dex-bNH 2 as well as 3.2 and $5.8 \mathrm{mV}$ for the Dex-TA $10^{-}$ $\left(\mathrm{bNH}_{2}\right)_{6}$ and Dex-TA $15-\left(\mathrm{bNH}_{2}\right)_{6}$, respectively. The HA-TA 2.2 showed an average aggregate size of $11.1 \mathrm{~nm}(\mathrm{pH} 6$ at $0.3 \mathrm{wt} \%)$, and a surface charge density of $-3.0 \mathrm{mV}$ (Table 4.1).

\subsubsection{Hydrogels properties}

The Horseradish peroxidase crosslinking of polysaccharide tyramine conjugates in the presence of hydrogen peroxide was shown a highly efficient method to prepare hydrogels. Hydrogels/cell constructs could be conveniently prepared at very low concentrations of Horseradish peroxidase $(15$ units $/ \mathrm{mL})$ and $\mathrm{H}_{2} \mathrm{O}_{2}(0.01 \mathrm{M})$ and showed good cytocompatibility [27]. At these $\mathrm{HRP}$ and $\mathrm{H}_{2} \mathrm{O}_{2}$ concentrations the gelation times of the different conjugates at concentrations of $5 \mathrm{wt} \%$ and $10 \mathrm{wt} \%$ were determined by the vial tilting method. The enzymatic crosslinking led to fast gelation within 6-15 seconds for all materials tested (Table 4.2). The presence of free amine groups appeared 
to have no influence on the gelation time and in control experiments $\mathrm{Dex}-\mathrm{bNH}_{2}$ did not react under these conditions. Such fast gelation makes these conjugates highly suitable as injectable scaffolds for various applications.

Table 4.2 Physical and mechanical properties of dextran and dextran/hyaluronic acid hydrogels

\begin{tabular}{|c|c|c|c|c|c|}
\hline Entry & $\mathrm{DS}^{a}$ & $\begin{array}{l}\text { Gelation } \\
\text { time }^{b} \\
(\mathrm{~s})\end{array}$ & $\begin{array}{l}\text { Swelling } \\
\text { ratio }\end{array}$ & $\begin{array}{c}\text { Storage } \\
\text { modulus } \\
(\mathrm{kPa})\end{array}$ & $\begin{array}{c}\text { Loss } \\
\text { modulus } \\
(\mathrm{kPa})\end{array}$ \\
\hline Dex-TA & 10 & $16 \pm 1$ & $8.2 \pm 0.2$ & $3.8 \pm 0.02$ & $0.12 \pm 0.01$ \\
\hline Dex-TA & 15 & $9 \pm 1$ & $6.6 \pm 0.1$ & $4.2 \pm 0.01$ & $0.23 \pm 0.01$ \\
\hline Dex-TA-bNH ${ }_{2}$ & $10: 6$ & $17 \pm 1$ & $8.1 \pm 0.1$ & $3.1 \pm 0.54$ & $0.14 \pm 0.05$ \\
\hline Dex-TA-bNH 2 & $15: 6$ & $9 \pm 1$ & $7.5 \pm 0.1$ & $4.0 \pm 0.03$ & $0.23 \pm 0.04$ \\
\hline $\begin{array}{l}\text { Dex-TA/HA-TA } \\
50 / 50\end{array}$ & $\begin{array}{l}15 / \\
2.2\end{array}$ & $8 \pm 1$ & $6.9 \pm 0.2$ & $4.1 \pm 0.07$ & $0.05 \pm 0.01$ \\
\hline
\end{tabular}

${ }^{a}$ : DS values were determined from ${ }^{1} \mathrm{H}$ NMR spectra;

${ }^{b}$ : determined by the vial tilting method.

The degree of swelling of the Dex-TA or Dex-TA-bNH $\mathrm{H}_{2}$ hydrogels in PBS was determined after $72 \mathrm{~h}$ incubation in water. In general, the gels showed degrees of swelling ranging from 6.6 to $8.2 \%$, values similar to previously determined values of Dextran-tyramine conjugates [9]. Moreover, the presence of the free amine groups $\left(\right.$ Dex-TA-bNH $\left.H_{2}\right)$ or free carboxylic acid groups (Dex-TA $\left.15 / \mathrm{HA}-\mathrm{TA}_{2.2}(50 / 50)\right)$ had no large effects on the swelling ratios.

The mechanical properties of $10 \mathrm{wt} \%$ Dex-TA or Dex-TA-bNH $\mathrm{H}_{2}$ hydrogels were investigated by oscillatory rheology measurements at $37{ }^{\circ} \mathrm{C}$. As shown in Table II, storage moduli of hydrogels prepared from dextran with conjugated tyramine and butyl amine groups showed no significant difference compared to tyramine conjugated dextran. Hydrogels were prepared at a low $\mathrm{H}_{2} \mathrm{O}_{2} /$ TA molar ratio of 0.1 , and at this low hydrogen peroxide concentration a storage modulus of $4.2 \mathrm{kPa}$ was determined for a Dex-TA 15 hydrogel. Hydrogels composed of Dex-TA $15 /$ HA-TA $2.2(50 / 50)$ gave a similar 
storage modulus but much lower loss modulus, which indicated that more elastic hydrogels were formed.

\subsubsection{Imaging of NIR labeled hydrogels}

The free amine groups present in the dextran conjugate Dex-TA-bNH 2 provided reactive groups that were used for the coupling of the NHS ester of the IRdye ${ }^{\circledR} 800 \mathrm{CW}$ (Scheme 4.2). This fluorescent dye has an excitation maximum at $787 \mathrm{~nm}$ and an emission maximum at $808 \mathrm{~nm}$. The conjugation of the dye was performed overnight at room temperature in the dark, and the product was purified by dialysis. Because noninvasive imaging of this fluorescent label is best performed at a concentration of $\sim 20$ $\mathrm{nM}$, and assuming quantitative coupling of the label, a series of calibration curves were prepared based on a quantitative coupling of the fluorescent label (Figure 4.3 A). The intensity of the Dex-TA $15-(\mathrm{bNH}-\mathrm{NIR})_{6}$ signal with concentration was linear and the calculated concentration corresponded to the found concentration. Addition of a gel precursor solution had almost no influence on the intensity of the signal. However, upon crosslinking a precursor solution of Dex-TA $15 / \mathrm{HA}-\mathrm{TA}_{2.2}(50 / 50)$ containing the Dex$\mathrm{TA}_{15}-\mathrm{b}\left(\mathrm{NH}_{2}\right)_{6}-(\mathrm{NIR})$ with HRP in the presence of $\mathrm{H}_{2} \mathrm{O}_{2}$, dramatically decreased the signal intensity.

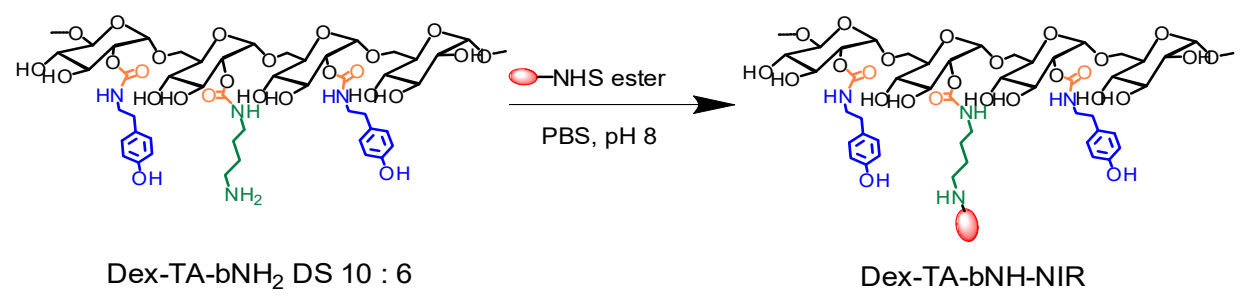

Scheme 4.2 Conjugation of IRdye ${ }^{\circledR} 800 \mathrm{CW}$ to Dex-TA $15-\mathrm{b}\left(\mathrm{NH}_{2}\right)_{6}$. (

IR Dye ${ }^{\circledR} 800$ CW NHS ester.)

The radicals generated during crosslinking likely react with the dye resulting in modification with concomitant change in the conjugated structure resulting in the disappearance of fluorescence. Solutions comprising the gel precursor and cells showed a somewhat higher signal intensity, whereas similarly as described above the 
crosslinked cell/gel constructs showed a low signal. In order to retain a sufficient signal for monitoring gels in vivo after implantation in mice, the concentration of the NIR dye conjugate in the hydrogels was increased to $100 \mathrm{nM}$. The quenching or stability of the NIR fluorescence dye was monitored in time up to $144 \mathrm{~h}$ storage in the dark. Although at high concentrations of 100 and $150 \mathrm{nM}$ the signal intensity was reduced by $15-18 \%$, almost no fluorescence was lost at low concentrations (Figure 4.3 C).

(A)

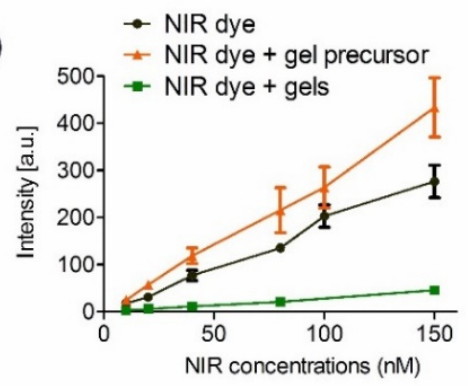

(B)

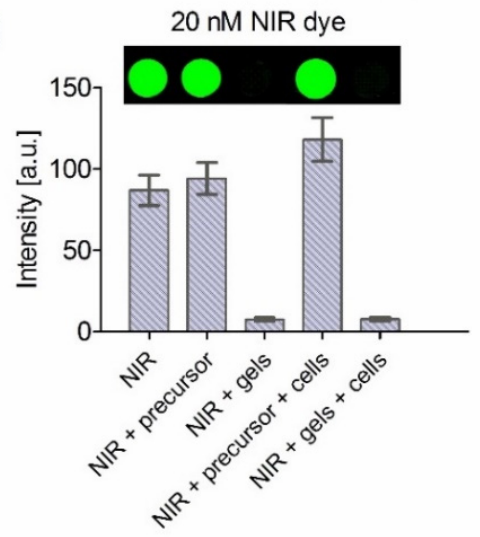

(C)

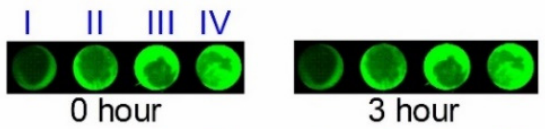

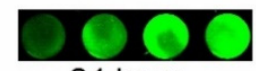

24 hour

I: $40 \mathrm{nM}$

150

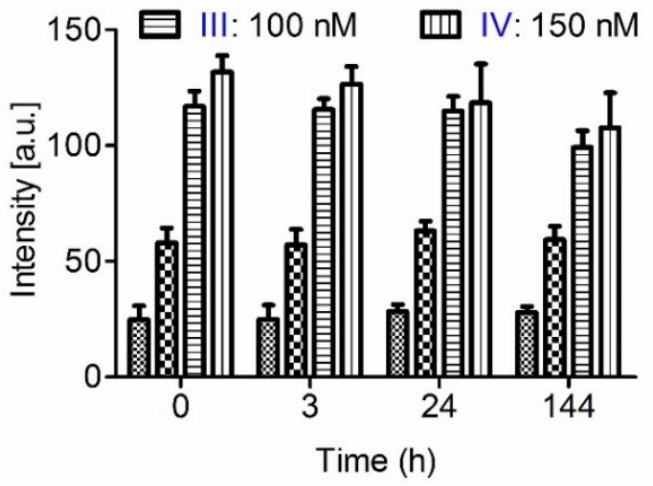

Figure 4.3 (A): Fluorescence calibration curves of NIR labeled conjugate solutions without or with a gel precursor solution and in hydrogels. (B): influence of gel precursor solution, gels or cells on the fluorescence intensity values and corresponding images on top. (C): stability of the NIR dye labeled gels in time. Intensity values of labeled gels as a function of NIR dye concentration and incubation time (images on top), respectively. Gels composition: $10 \mathrm{wt} \%$, Dex-TA $15 / \mathrm{HA}-\mathrm{TA}_{2.2}(50 / 50)$.

\subsubsection{Tracking the degradation by in vivo imaging}


To evaluate the effect of local enzyme mediated biodegradation profile in vivo, Dex$\mathrm{TA}_{15}$ or Dex-TA $15 / \mathrm{HA}-\mathrm{TA}_{2.2}$ (50/50) hydrogels with or without cells were subcutaneously implanted into 4 different pockets of mice (Figure 4.4). Typically, to the precursor solutions $\left(10 \mathrm{wt} \%\right.$, Dex-TA ${ }_{15}$ or Dex-TA $\left.15 / \mathrm{HA}-\mathrm{TA}_{2.2}(50 / 50)\right)$ the NIRfunctionalized Dex-TA $15-(\mathrm{bNH}-\mathrm{NIR})_{6}$ was added at a final concentration of $100 \mathrm{nM}$. Hydrogels were prepared by crosslinking solutions with HRP (15 units $/ \mathrm{mL}$ ) and $\mathrm{H}_{2} \mathrm{O}_{2}$ $(0.01 \mathrm{M})$. Hydrogels with cells $\left(\mathrm{hMsc} / \mathrm{bCh}, 80 / 20,10 \times 10^{6} / \mathrm{mL}\right)$ were prepared in a similar way. Hydrogels were washed with PBS and stored in the dark before implantation. Images of the fluorescent labeled hydrogels were taken at regular time intervals up to 10 weeks using a PEARL impulse imaging camera.

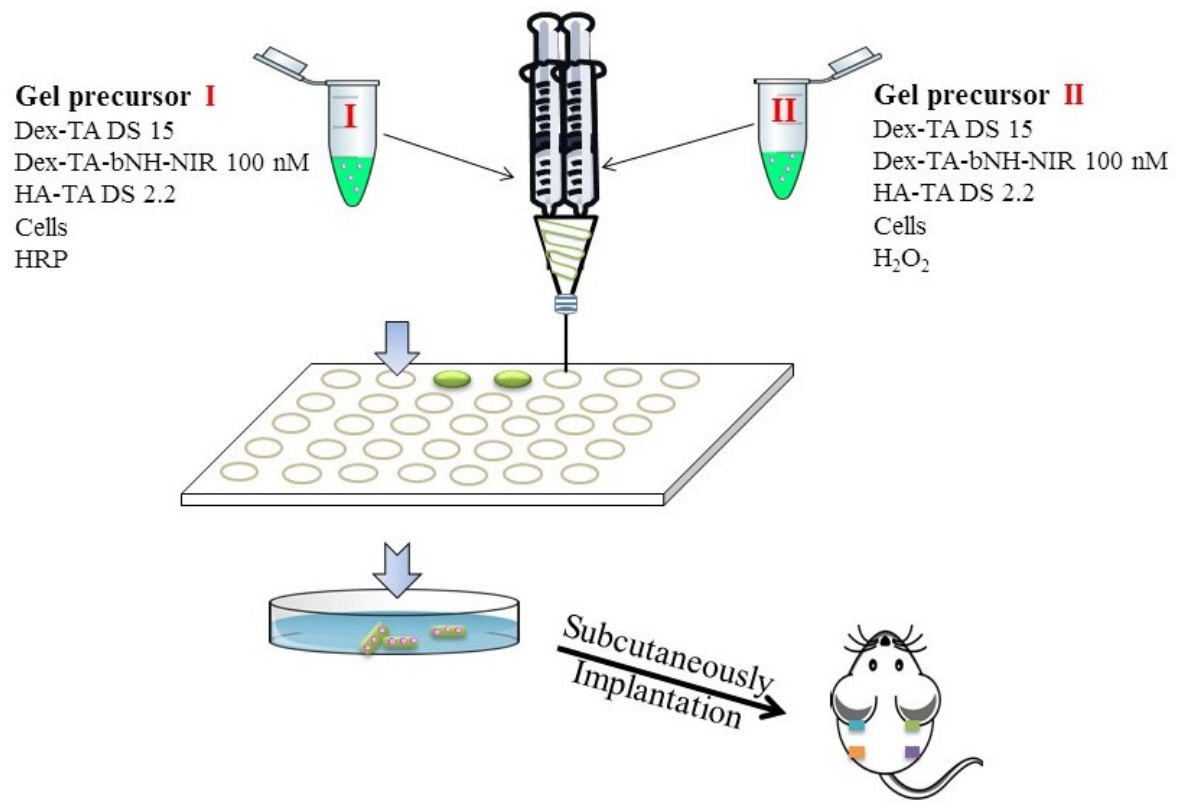

Figure 4.4 Schematic representation of subcutaneous implantation of NIR labeled hydrogels in mice.

A typical mouse image, fluorescence image and an overlay image are presented in Figure 4.5 top. The time dependent fluorescence signal intensities determined for different hydrogels with or without cells, as presented in Figure 4.5 clearly show a decrease in the NIR fluorescence signal for the Dex- $\mathrm{TA}_{15} / \mathrm{HA}-\mathrm{TA}_{2.2}(50 / 50)$ hydrogel constructs with or without cells over the implantation time (week 2 to week 10). Non- 
macroscopic signal changes can be observed for Dex-TA 15 hydrogels with or without cells. To further quantify the NIR fluorescent signal loss, NIR intensity values measured were normalized against day 0 and are graphically presented in Figure 4.6. Interestingly all graphs revealed a steady decrease in signal intensities in time. This decrease in somewhat larger for the Dex-TA $15 / \mathrm{HA}-\mathrm{TA}_{2.2}$ (50/50) hydrogels ( $60 \%$ loss) compared to Dex-TA 15 hydrogels (40 50\% loss) but there appears no significant influence of cells incorporated.
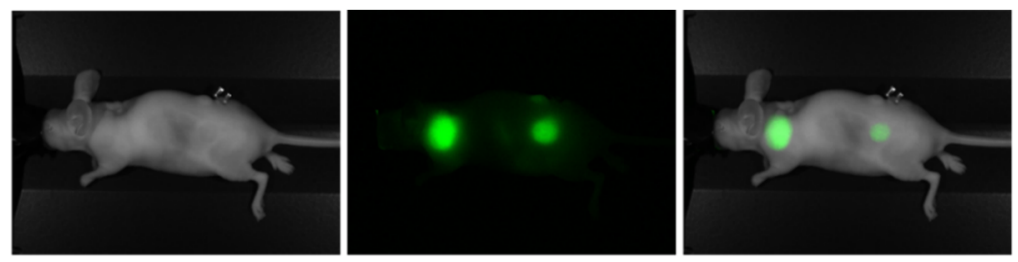

\section{no implant week 2 week 4 week 6 week 8 week 10}

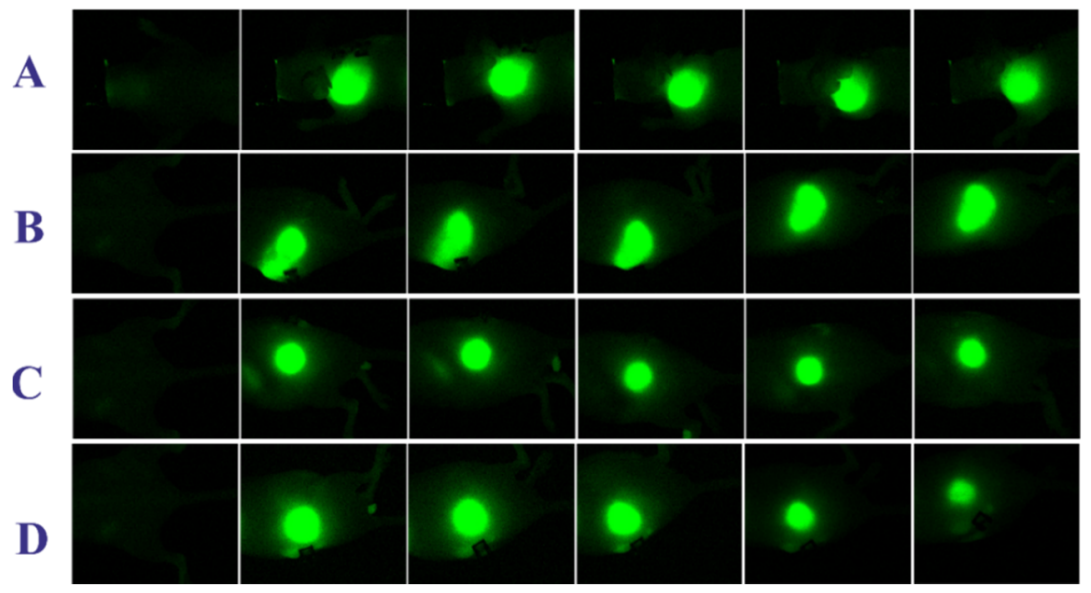

Figure 4.5 Top: images of a mouse, fluorescence signal and superposition. Fluorescence intensity imaging of a NIR labeled Dex-TA 15 hydrogel (A), a NIR labeled Dex-TA 15 hydrogel with hMsc/bCh (80/20) encapsulated (B), a NIR labeled Dex$\mathrm{TA}_{15} / \mathrm{HA}-\mathrm{TA}_{2.2}(50 / 50)$ hydrogel $(\mathrm{C})$ and a NIR labeled Dex-TA $15 / \mathrm{HA}-\mathrm{TA}_{2.2}(50 / 50)$ hydrogel with hMsc/bCh (80/20) encapsulated (D). 

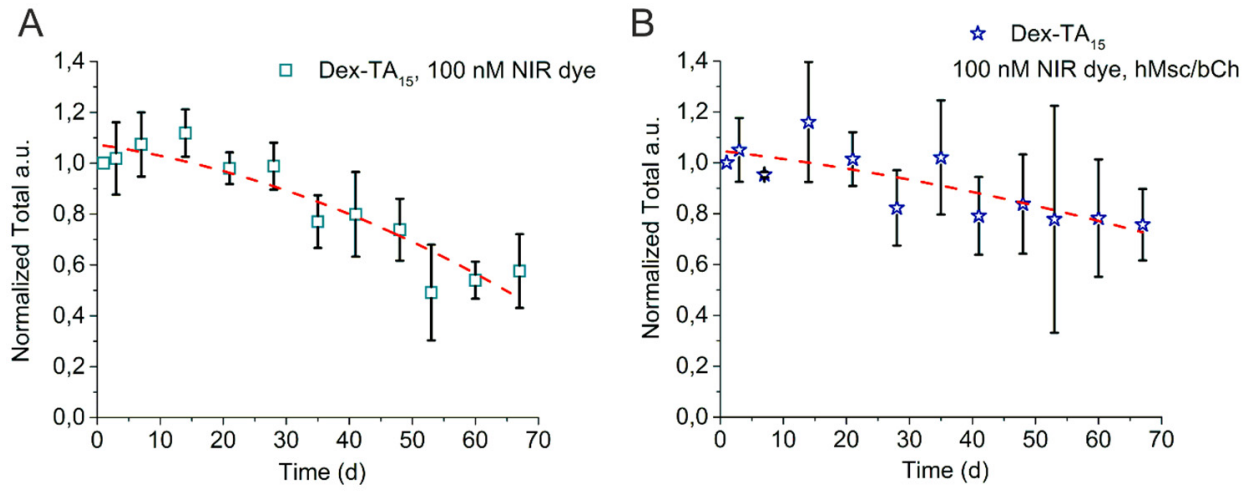

C

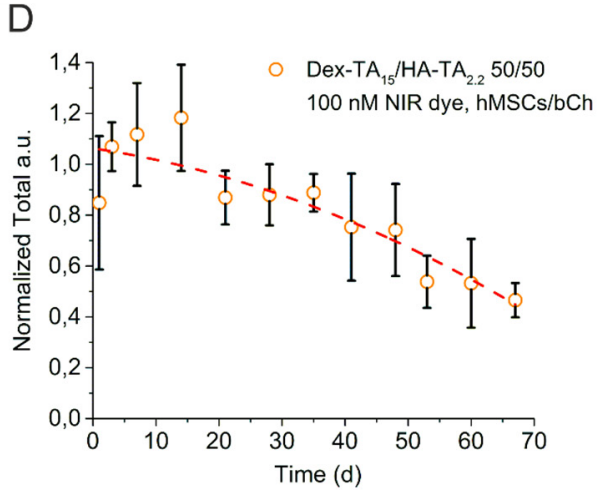

Figure 4.6 Normalized total fluorescence intensity as a function of time of Dex-TA 15 hydrogels without cells (A) and with hMSCs/bCh (80/20) (B). Normalized total fluorescence intensity as a function of time of Dex-TA $15 / \mathrm{HA}-\mathrm{TA}_{2.2}(50 / 50)$ hydrogels without cells (C) and with hMSCs/bCh (80/20) (D). Samples were imaged at an emission wavelength of $800 \mathrm{~nm}$. Hydrogels were prepared at $10 \mathrm{wt} \%$ and a NIR dye concentration of $100 \mathrm{nM}$. Cell density was $10 \times 10^{6} / \mathrm{mL}$. The dotted lines in the graphs are only a guide to the eye.

In previous research the in vitro degradation of Dex-TA ${ }_{15}$ and Dex-TA $15 / \mathrm{HA}-\mathrm{TA}_{2.2}$ (50/50) hydrogels in PBS containing 20 units/mL hyaluronidase was determined. The Dex-TA $15 /$ HA-TA $2.2(50 / 50)$ degraded in approximately 55 days, and no degradation was observed for Dex-TA 15 gels. This in vivo slow degradation profile may be due to much lower levels of hyaluronidase present at the implantation site compared to that used in the in vitro degradation study. However, the loss of the NIR signal for Dex-TA 15 gels indicated that chemical or enzymatic hydrolysis or a foreign body reaction may 
induce the degradation of the hydrogels. These results show that this NIR fluorescent imaging method may provide additional insight in in vivo degradation profiles in the long term. Particularly, the data show that no large differences were observed in the degradation rate for gels with or without cells incorporated. Histology of the explants, which is currently under investigation, may provide additional insight in processes regulating the degradation of these type of scaffolds and assess the biological performance of incorporated cells as well as their effect on the hydrogel degradation.

\subsection{Conclusions}

Non-invasive monitoring of degradable hydrogels using a near infrared fluorescent label allows an extensive long-term in vivo measurement of the degradation of hydrogels by imaging without the need to sacrifice large numbers of animals. A dextran co-conjugate comprising tyramine and free amine groups was successfully prepared and labeled with a NIR-dye. This conjugate was incorporated in tyramine conjugated dextran or dextran/hyaluronic acid hydrogels upon enzymatic crosslinking. In this study it was shown that these NIR labeled enzymatically crosslinkable hydrogels can be monitored by a non-invasive procedure over a 70 days period. The decrease in fluorescence revealed the degradation of the gels in time and appeared independent on the presence of hMSCs/bCh incorporated.

\section{References}

[1] Van Tomme, S. R.; Storm, G.; Hennink, W. E., In situ gelling hydrogels for pharmaceutical and biomedical applications. Int. J. Pharm. 2008, 355 (1-2), 1-18.

[2] Yu, L.; Ding, J., Injectable hydrogels as unique biomedical materials. Chem. Soc. Rev. 2008, 37 (8), 1473-1481.

[3] Singh, N. K.; Lee, D. S., In situ gelling pH- and temperature-sensitive biodegradable block copolymer hydrogels for drug delivery. J. Controlled Release 2014, 193, 214-227. 
[4] Cai, S. S.; Liu, Y. C.; Shu, X. Z.; Prestwich, G. D., Injectable glycosaminoglycan hydrogels for controlled release of human basic fibroblast growth factor. Biomaterials 2005, 26 (30), 6054-6067.

[5] Anseth, K. S.; Metters, A. T.; Bryant, S. J.; Martens, P. J.; Elisseeff, J. H.; Bowman, C. N., In situ forming degradable networks and their application in tissue engineering and drug delivery. J. Controlled Release 2002, 78 (1-3), 199-209.

[6] Patenaude, M.; Smeets, N. M. B.; Hoare, T., Designing Injectable, Covalently Cross- Linked Hydrogels for Biomedical Applications. Macromol. Rapid Commun. 2014, 35 (6), 598-617.

[7] Wang, Y.; Cai, L.-Q.; Nugraha, B.; Gao, Y.; Leo, H. L., Current Hydrogel Solutions for Repairing and Regeneration of Complex Tissues. Curr. Med. Chem. 2014, 21 (22), 2480-2496.

[8] Sofia, S. J.; Singh, A.; Kaplan, D. L., Peroxidase-catalyzed crosslinking of functionalized polyaspartic acid polymers. J. macromol. Sci. A 2002, 39 (10), 11511181.

[9] Jin, R.; Hiemstra, C.; Zhong, Z. Y.; Feijen, J., Enzyme-mediated fast in situ formation of hydrogels from dextran-tyramine conjugates. Biomaterials 2007, 28 (18), 2791-2800.

[10] Jin, R.; Teixeira, L. S. M.; Dijkstra, P. J.; Karperien, M.; van Blitterswijk, C. A.; Zhong, Z. Y.; Feijen, J., Injectable chitosan-based hydrogels for cartilage tissue engineering. Biomaterials 2009, 30 (13), 2544-2551.

[11] Jin, R.; Teixeira, L. S. M.; Dijkstra, P. J.; van Blitterswijk, C. A.; Karperien, M.; Feijen, J., Enzymatically-crosslinked injectable hydrogels based on biomimetic dextranhyaluronic acid conjugates for cartilage tissue engineering. Biomaterials 2010, 31 (11), 3103-3113.

[12] Park, K. M.; Lee, Y.; Son, J. Y.; Oh, D. H.; Lee, J. S.; Park, K. D., Synthesis and Characterizations of In Situ Cross-Linkable Gelatin and 4-Arm-PPO-PEO Hybrid Hydrogels via Enzymatic Reaction for Tissue Regenerative Medicine. Biomacromolecules 2012, 13 (3), 604-611.

[13] Lee, Y.; Bae, J. W.; Oh, D. H.; Park, K. M.; Chun, Y. W.; Sung, H. J.; Park, K. D., In situ forming gelatin-based tissue adhesives and their phenolic content-driven properties. J. Mater. Chem. B 2013, 1 (18), 2407-2414. 
[14] Park, K. M.; Jun, I.; Joung, Y. K.; Shin, H.; Park, K. D., In situ hydrogelation and RGD conjugation of tyramine-conjugated 4-arm PPO-PEO block copolymer for injectable bio-mimetic scaffolds. Soft Matter 2011, 7 (3), 986-992.

[15] Park, K. M.; Lee, Y.; Son, J. Y.; Bae, J. W.; Park, K. D., In Situ SVVYGLR Peptide Conjugation into Injectable Gelatin-Poly(ethylene glycol)-Tyramine Hydrogel via Enzyme-Mediated Reaction for Enhancement of Endothelial Cell Activity and NeoVascularization. Bioconjugate Chem. 2012, 23 (10), 2042-2050.

[16] Gohil, S. V.; Brittain, S. B.; Kan, H.-M.; Drissi, H.; Rowe, D. W.; Nair, L. S., Evaluation of enzymatically crosslinked injectable glycol chitosan hydrogel. J. Mater. Chem. B 2015, 3 (27), 5511-5522.

[17] Buwalda, S. J.; Boere, K. W. M.; Dijkstra, P. J.; Feijen, J.; Vermonden, T.; Hennink, W. E., Hydrogels in a historical perspective: From simple networks to smart materials. J. Controlled Release 2014, 190, 254-273.

[18] Artzi, N.; Oliva, N.; Puron, C.; Shitreet, S.; Artzi, S.; Ramos, A. B.; Groothuis, A.; Sahagian, G.; Edelman, E. R., In vivo and in vitro tracking of erosion in biodegradable materials using non-invasive fluorescence imaging. Nat. Mater. 2011, 10 (9), 704-709.

[19] Wang, W.; Liu, J.; Li, C.; Zhang, J.; Liu, J.; Dong, A.; Kong, D., Real-time and non-invasive fluorescence tracking of in vivo degradation of the thermosensitive PEGlyated polyester hydrogel. J. Mater. Chem. B 2014, 2 (26), 4185-4192.

[20] Stevenson Jr, A. T.; Reese, L. M.; Hill, T. K.; McGuire, J.; Mohs, A. M.; Shekhar, R.; Bickford, L. R.; Whittington, A. R., Fabrication and characterization of medical grade polyurethane composite catheters for near-infrared imaging. Biomaterials 2015, 54, 168-176.

[21] Kim, S. H.; Lee, J. H.; Hyun, H.; Ashitate, Y.; Park, G.; Robichaud, K.; Lunsford, E.; Lee, S. J.; Khang, G.; Choi, H. S., Near-Infrared Fluorescence Imaging for Noninvasive Trafficking of Scaffold Degradation. Sci. Rep. 2013, 3 (1198), 1-7.

[22] Jalani, G.; Naccache, R.; Rosenzweig, D. H.; Lerouge, S.; Haglund, L.; Vetrone, F.; Cerruti, M., Real-time, non-invasive monitoring of hydrogel degradation using LiYF4:Yb3+/Tm3+ NIR-to-NIR upconverting nanoparticles. Nanoscale 2015, 7 (26), $11255-11262$. 
[23] Hendriks, J.; Riesle, J.; Van Blitterswijk, C. A., Effect of stratified culture compared to confluent culture in monolayer on proliferation and differentiation of human articular chondrocytes. Tissue Eng. 2006, 12 (9), 2397-2405.

[24] Fernandes, H.; Dechering, K.; Van Someren, E.; Steeghs, I.; Apotheker, M.; Leusink, A.; Bank, R.; Janeczek, K.; Van Blitterswijk, C.; de Boer, J., The Role of Collagen Crosslinking in Differentiation of Human Mesenchymal Stem Cells and MC3T3-E1 Cells. Tissue Eng. Part A 2009, 15 (12), 3857-3867.

[25] Antoniou, E.; Tsianou, M., Solution properties of dextran in water and in formamide. J. Appl. Polym. Sci. 2012, 125 (3), 1681-1692.

[26] Antoniou, E.; Buitrago, C. F.; Tsianou, M.; Alexandridis, P., Solvent effects on polysaccharide conformation. Carbohydr. Polym. 2010, 79 (2), 380-390.

[27] Jin, R.; Dijkstra, P. J.; Feijen, J., Rapid gelation of injectable hydrogels based on hyaluronic acid and poly(ethylene glycol) via Michael-type addition. J. Controlled Release 2010, 148 (1), E41-E43. 


\section{Chapter 5}

Enzymatic co-crosslinking of star-shaped poly(ethylene glycol) tyramine and hyaluronic acid tyramine conjugates provides elastic biocompatible and biodegradable hydrogels

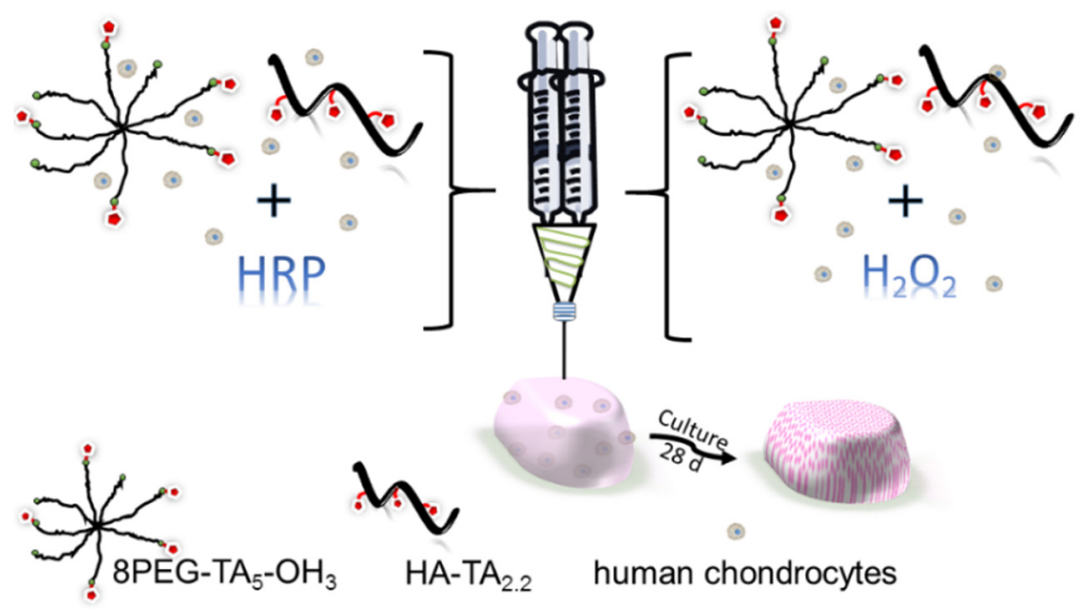

* This chapter is in preparation: Rong Wang, Xiaobin Huang, Pieter J. Dijkstra and Marcel Karperien. 2015. 


\section{Abstract}

Combining the viscoelastic properties of hyaluronic acid (HA) and the elastic properties of star shaped 8 arm poly(ethylene glycol) (8PEG) was used to design in-situ forming hydrogels. Hydrogels were prepared by the enzymatic crosslinking of a partially tyramine end group modified $8 P E G$ and a tyramine conjugated HA using horseradish peroxidase in the presence of hydrogen peroxide. Hydrogels of the homopolymer conjugates and mixtures thereof were rapidly formed within seconds under physical conditions at low polymer and enzyme concentrations. Elastic hydrogels with high gel content $(\geq 95 \%$ ) and high storage moduli (up to22.4 Kpa) were obtained. An in vitro study in the presence of hyaluronidase (100 U/mL) revealed that with increasing PEG content the degradation of the hybrid hydrogels decreased up to several weeks, whereas hydrogels composed of only hyaluronic acid degraded within 2 weeks. Human mesenchymal stem cells (hMSCs) incorporated in the hybrid hydrogels remained viable as shown by a PrestoBlue and a live-dead assay, indicating the biocompatibility of the constructs. The production of an extra cellular matrix by redifferentiation of encapsulated human chondrocytes was followed over a period of $28 d$. Gene expression indicated that these highly elastic hydrogels induced an enhanced production of collagen type II. At low 8PEG-TA/HA-TA ratios a higher expression of SOX 9 and ACAN was observed. These results indicate that by modulating the ratio of 8PEG/HA tyramine conjugates, injectable hydrogels can be prepared applicable as scaffolds for tissue regeneration applications. 


\subsection{Introduction}

In-situ forming hydrogels prepared by the enzymatic crosslinking of polymer conjugates received increasing attention for tissue regeneration applications during the past decade. Especially, the enzyme horseradish peroxidase which has a high substrate specificity for phenolic residues present in tyramine and tyrosine was extensively studied. Up to now the crosslinking of polymer conjugates was mainly directed to natural polysaccharides like e.g. dextran, hyaluronic acid, heparin and chitosan [1-14]. The fast covalent coupling of tyramine residues through radical generation by the enzyme in the presence of hydrogen peroxide made in-situ hydrogel formation possible within seconds and these systems are nowadays also named injectable hydrogels. Although concerns on the use of this plant enzyme and hydrogen peroxide have been posed, the very low concentrations of the catalyst and reagent necessary revealed no cytotoxic effects on cells in contact with the hydrogels or when cells were embedded into the hydrogel constructs $[15,16]$.

The versatility of the system can be illustrated by more recent studies, which are on the basis of the work of Kaplan and co-workers [17] who showed that poly( $\gamma$-glutamine acid) conjugated with tyramine or tyrosine were rapidly crosslinked into hydrogels by $\mathrm{HRP} / \mathrm{H}_{2} \mathrm{O}_{2}$. Based on these results tyramine has become the main choice for end-group modification of synthetic water soluble polymers or grafted to natural polymers. Concerning the latter, next to dextran, hyaluronic acid and chitosan tyramine conjugates, alginate, celluloses, and proteins like gelatin and albumin were studied for their application as injectable hydrogels [18-27]. Natural polymers containing carboxylic acid groups were conjugated with tyramine after activation methods and polymers containing free amine groups, like in chitosan or gelatin, conjugation is performed with an activated hydroxyphenyl alkanoic acid.

Synthetic water soluble polymers conjugated or end-functionalized with tyramine groups received minor attention up to now. Sakai and co-workers studied hydrogels based on tyramine conjugates of poly(vinyl alkohol) as wound dressing materials [28]. They developed a method for the in-situ generation of hydrogen peroxide by the enzymatic oxidation reaction of glucose in the present of glucose oxidase. Controlling the concentration of the hydrogen peroxide during the crosslinking process is beneficial 
because HRP is inactivated at relative high concentrations of $\mathrm{H}_{2} \mathrm{O}_{2}$. Park and coworkers described the use of tyramine coupled to end-functionalized four arm poly(propylene oxide)-poly(ethylene oxide) copolymers. These materials could be degraded via hydrolysis of labile esters linking units next to the PEO chain. Linear mono tyramine end-functionalized PEGs have been grafted to gelatin and hydrogels were prepared by co-crosslinking with hyaluronic acid. Importantly, it was also shown in these studies that peptides containing tyrosine residues can be co-crosslinked in the networks providing bioactivity to the hydrogels [24-26,29,30].

The highly efficient and rapid enzymatic crosslinking reaction of polymer tyramine conjugates triggered researchers in studies towards the supramolecular engineering of hydrogels. Inclusion complexes of cyclodextrin and PEG were co-crosslinked with chitosan providing materials with controlled degradation profiles [31]. In a paper by the group of Haag and co-workers the temperature dependency on the kinetics of the crosslinking reaction of 3-(4-hydroxyphenyl)propionic acid (HPA) functionalized hyperbranced polyglycerol (hPG) was shown. They showed that the enzyme concentration and temperature could be used as tools to control the reaction kinetics and thereby the ultimate mechanical properties of the hydrogel [32].

Hyaluronic acid is an important component of the extracellular matrix in many tissues and is an attractive material for a variety of biomedical applications. HA plays a central role in protein adhesion and provides attachment sites for e.g. chondrocytes. Hydrogels comprising HA generally show viscoelastic properties, good water retention, and interaction with cells [33-35]. Synthetic hydrophilic polymers like PEGs with controlled molecular weight and end-groups are nowadays commercially available. Among the large number of PEGs nowadays available, the star shaped 8 arm PEG has shown a versatile hydrophilic component in a variety of physically and chemically crosslinked hydrogels and is generally elastic. Combining the viscoelastic properties and cell interactions of hyaluronic acid and the generally elastic properties and controlled end group modification of the star shaped 8 arm poly(ethylene glycol) (8PEG) was regarded favorable considering in-situ forming hydrogels in tissue regeneration applications. These properties might create a suitable compatible environment for cells and when combined with controlled degradation in time a more optimal regeneration of intended tissues. Already in previous research it was shown that HA can be conjugated 
with tyramine groups. The 8 arm PEG containing hydroxyl end-groups was selected for its long-term in vivo inertness and possible tyramine coupling after activation with a chloroformate. The preparation of enzymatically crosslinked hydrogels based on these PEG-tyramine and HA-tyramine conjugates was investigated in the presence of HRP and $\mathrm{H}_{2} \mathrm{O}_{2}$. The properties of hydrogels such as gelation time, gel content, swelling/degradation and mechanical properties were studied. Moreover, human mesenchymal stem cells were incorporated in these hydrogels to evaluate their cytocompatibility and human chondrocytes were incorporated to evaluate their proliferation and differentiation.

\subsection{Materials and methods}

\subsubsection{Materials}

The 8 arm poly(ethylene glycol) (8PEG, hexaglycerol core, $\mathrm{Mw}=20,000$ ) was acquired from Jenkem Technology (Allen, Texas, USA) and purified before use by dissolution in dichloromethane and precipitation in cold diethyl ether. Hyaluronic acid sodium salt $(\mathrm{Mw}=15$ to $25 \mathrm{~kg} / \mathrm{mol})$ was purchased from Contipro. The polymer was freeze dried overnight before use. $p$-Nitrophenyl chloroformate (PNC, 96\%) was purchased from Sigma-Aldrich (Zwijndrecht, the Netherlands) and was sublimated before use. Anhydrous dimethylformamide (DMF, 99.8\%), tyramine (99\%), anhydrous pyridine (99.8\%), hydrogen peroxide $\left(\mathrm{H}_{2} \mathrm{O}_{2}, 30 \mathrm{wt} \%\right.$ in $\left.\mathrm{H}_{2} \mathrm{O}\right)$, lithium chloride and $\mathrm{HRP}$ (261 purpurogallin units $/ \mathrm{mg}$ solid) were obtained from Sigma-Aldrich and used without further purification. Dichloromethane (stabilized by Amylene, $\geq 99.9 \%$ ), ethanol (99.8\%) and diethyl ether (stabilized by BHT, $\geq 99.5 \%$ ) were from Biosolve (Valkenswaard, The Netherlands) and used as received. Dialysis membranes (Spectra/Por molecular porous membrane tubing 6, MWCO: $1 \mathrm{kD}$ ) were acquired from Spectrum Laboratories, Inc. (Breda, The Netherlands) and rinsed with distilled water before use. Deuterated solvents were purchased from Aldrich. Phosphate buffered saline (PBS, $150 \mathrm{mM}, \mathrm{pH}$ 7.4) was purchased from Gibco Life Technologies Ltd (Paisley, UK).

\subsubsection{Synthesis}


PEG-OH ${ }_{8}$ was firstly activated with p-nitrophenyl chloroformate (PNC) to form $p$ nitrophenyl carbonate conjugates (PEG-PNC $5-\mathrm{OH}_{3}$ ) as previously reported [36]. Hyaluronic acid-tyramine (HA-TA) was prepared as reported previously [8].

PEG-TA5-OH3. Typically, PEG-PNC $5-\mathrm{OH}_{3}(5.0 \mathrm{~g}, 1.2 \mathrm{mmol})$ was dissolved in $50 \mathrm{~mL}$ of anhydrous dichloromethane, and then tyramine $(0.33 \mathrm{~g}, 2.4 \mathrm{mmol})$ pre-dissolved in 5 $\mathrm{mL}$ of anhydrous DMF was added at room temperature. The solution was stirred under a $\mathrm{N}_{2}$ atmosphere for $2 \mathrm{~h}$. The product was precipitated in cold diethyl ether, followed by washing with cold ethanol and diethyl ether and then dried under vacuum for one day to give PEG-TA $5-\mathrm{OH}_{3}$ (yield 92\%). ${ }^{1} \mathrm{H}$ NMR (400 MHz, $\mathrm{CDCl}_{3}$ ): $\delta=2.7\left(\mathrm{t}, \mathrm{NHCH}_{2} \mathrm{CH}_{2}\right.$ ), 3.3-3.4 (dd, $\mathrm{NHCH}_{2} \mathrm{CH}_{2}$ ), 3.63 (PEG protons), 4.19 (t, $\mathrm{CH}_{2} \mathrm{OCO}$ ), 5.00 (s, NH), 6.796.99 (d, aromatic protons).

\subsubsection{Analysis}

NMR spectroscopy: NMR spectra were recorded on a Bruker Ascend ${ }^{\mathrm{TM}}$ III 400 NMR (Nanobay) Spectrometer working at $400.13 \mathrm{MHz}$ for ${ }^{1} \mathrm{H}$. The chemical shifts were calibrated against residual solvent signals. Peak multiplicity was denoted as s (singlet), d (doublet), dd (double doublet) and t (triplet).

Hydrogel formation and gelation time. Enzymatically cross-linked hydrogels $(0.2 \mathrm{~mL})$ of PEG-TA $-\mathrm{OH}_{3} / \mathrm{HA}-\mathrm{TA}_{2.2}(50 / 50)$ at a polymer concentration of 5, 10 or $20 \mathrm{wt} \%$ were prepared in vials at room temperature. The gelation time vs enzyme concentration was evaluated by changing HRP concentrations. In a typical procedure, to $0.16 \mathrm{~mL}$ of PEG$\mathrm{TA}_{5}-\mathrm{OH}_{3} / \mathrm{HA}-\mathrm{TA}_{2.2}(50 / 50,12.5 \mathrm{wt} \%)$ in PBS, a freshly prepared HRP solution $(20 \mu \mathrm{L}$, $25 \mathrm{unit} / \mathrm{mL})$ and $\mathrm{H}_{2} \mathrm{O}_{2}$ solution $(20 \mu \mathrm{L}, 0.3 \mathrm{wt} \%$ stock solution) were added and the mixture was gently vortexed. The final polymer, HRP and $\mathrm{H}_{2} \mathrm{O}_{2}$ concentrations were 10 $\mathrm{wt} \%, 2.5 \mathrm{unit} / \mathrm{mL}$ and $0.01 \mathrm{M}$, respectively. The gelation time was determined by using the vial tilting method. No flow within 5 min upon inverting the vial was regarded as the gel state. The experiment was performed in triplicate.

Gel content and water uptake: To determine the gel content, samples of PEG-TA5$\mathrm{OH}_{3}, \mathrm{HA}-\mathrm{TA}_{2.2}$ or PEG-TA $-\mathrm{OH}_{3} / \mathrm{HA}-\mathrm{TA}_{2.2}$ hydrogels $(0.2 \mathrm{~mL}, 10 \mathrm{wt} \%)$ were prepared as described above. The gels were lyophilized and weighted $\left(\mathrm{W}_{\mathrm{d}}\right)$, then extensively extracted with $5 \mathrm{~mL}$ of dichloromethane for a week to remove un-crosslinked polymer. 
The solvent was replaced twice. The hydrogel was washed 3 times with ethanol and dried under vacuum to a constant weight $\left(\mathrm{W}_{\mathrm{c}}\right)$. The gel content was expressed as $\mathrm{W}_{\mathrm{c}} / \mathrm{W}_{\mathrm{d}} \times 100 \%$.

The water uptake of PEG-TA $5-\mathrm{OH}_{3}, \mathrm{HA}-\mathrm{TA}_{2.2}$ or PEG-TA ${ }_{5}-\mathrm{OH}_{3} / \mathrm{HA}-\mathrm{TA}_{2.2}$ hydrogels $(0.2 \mathrm{~mL}, 10 \mathrm{wt} \%)$ was determined as follows. After lyophilization, the dry hydrogels $\left(\mathrm{W}_{\mathrm{d}}\right)$ were immersed in $5 \mathrm{~mL}$ of $\mathrm{PBS}$ at $37{ }^{\circ} \mathrm{C}$ for $3 \mathrm{~d}$ in order to reach swelling equilibrium. Swollen samples were removed from PBS and after removal of surface water, the samples were weighted $\left(\mathrm{W}_{\mathrm{s}}\right)$. The water uptake of the hydrogels was calculated as follows: $\left(\mathrm{W}_{\mathrm{s}}-\mathrm{W}_{\mathrm{d}}\right) / \mathrm{W}_{\mathrm{d}} \times 100 \%$.

Morphology of gels: In order to visualize the morphology of PEG-TA $5-\mathrm{OH}_{3}, \mathrm{HA}-\mathrm{TA}_{2.2}$ or PEG-TA $-\mathrm{OH}_{3} / \mathrm{HA}-\mathrm{TA}_{2.2}$ hydrogels, the samples were frozen in liquid nitrogen and then dried for two days. After coating with gold, samples were analyzed with a HRSEM-Zeiss scanning electron microscope (SEM) equipped with NORAN EDS and WDS with a maximum and minimum accelerating voltage of $30 \mathrm{kV}$ and $0.2 \mathrm{kV}$, respectively, and a field-emission.

Rheology: Oscillatory rheology experiments were performed on PEG-TA ${ }_{5}-\mathrm{OH}_{3}, \mathrm{HA}-$ $\mathrm{TA}_{2.2}$ or PEG-TA $-\mathrm{OH}_{3} / \mathrm{HA}-\mathrm{TA}_{2.2}$ hydrogels with a concentration of $10 \mathrm{wt} \%$. Hydrogels were in-situ formed using a MixPac ${ }^{\mathrm{TM}}$ double chamber syringe system with a Medmix Systems AG ML-2.0-16-LLM mixing chamber (Winterthur, Switzerland). In a typical experiment, a polymer solution $(0.16 \mathrm{~mL}, 12.5 \mathrm{wt} \%$ in PBS $)$ mixed with a freshly prepared solution of $\mathrm{H}_{2} \mathrm{O}_{2}(40 \mu \mathrm{L}, 0.3 \mathrm{wt} \%$ stock solution in PBS) was placed in one of the syringe chambers. Another polymer solution $(0.16 \mathrm{~mL}, 12.5 \mathrm{wt} \%$ in PBS) was mixed with a freshly prepared HRP solution ( $40 \mu \mathrm{L}, 25 \mathrm{unit} / \mathrm{mL}$ stock solution in PBS) and placed in the other chamber. After the sample was injected onto the bottom plate, the upper plate was immediately lowered to a measuring gap size of $0.3 \mathrm{~mm}$ followed by a time sweep for $10 \mathrm{~min}$ at $25^{\circ} \mathrm{C}$. A frequency $\omega$ of $1 \mathrm{~Hz}$ and a strain $\gamma$ of $1 \%$ were applied to minimize the influence of deformation on the hydrogels. Then, a strain sweep was recorded at a constant frequency $(\omega=1 \mathrm{~Hz} ; \gamma=0.1-50 \%)$ and after $10 \mathrm{~min}$ equilibration, a frequency sweep was recorded at constant strain amplitude $(\gamma=1 \%$; $\omega$ $=0.1-100 \mathrm{~Hz}$ ) at $25^{\circ} \mathrm{C}$.

Compression tests: Mechanical compression tests on PEG-TA $-\mathrm{OH}_{3}, \mathrm{HA}-\mathrm{TA}_{2.2}$ or PEG-TA $5-\mathrm{OH}_{3} / \mathrm{HA}-\mathrm{TA}_{2.2}$ hydrogels were carried out using a Zwick-Roell Z020 tester 
(Germany) with a load cell of 250 N. Standard cylindrical hydrogel samples were prepared using a PDMS substrate (4 $\mathrm{mm}$ height and $9 \mathrm{~mm}$ diameter). The specimens were placed between two loads, and the testing configuration was set to obtain the ultimate strength of specimens under compression. A compressive speed of $1 \mathrm{~mm} / \mathrm{s}$ was used. Six samples of each group were tested.

Swelling and degradation behavior: $\mathrm{PEG}-\mathrm{TA}_{5}-\mathrm{OH}_{3}, \mathrm{HA}-\mathrm{TA}_{2.2}$ or PEG-TA5 $-\mathrm{OH}_{3} / \mathrm{HA}-$ $\mathrm{TA}_{2.2}$ hydrogels $(0.2 \mathrm{~mL}, 10 \mathrm{wt} \%)$ were prepared as described above and accurately weighted $\left(\mathrm{W}_{\mathrm{i}}\right)$. The samples were subsequently incubated in $2 \mathrm{~mL}$ of PBS containing 100 units $/ \mathrm{mL}$ hyaluronidase at $37^{\circ} \mathrm{C}$. At regular time intervals, the enzyme solution was removed from the samples and the weight of the hydrogels was determined $\left(\mathrm{W}_{\mathrm{de}}\right)$. Remaining gel (\%) was calculated from $\mathrm{W}_{\mathrm{de}} / \mathrm{W}_{\mathrm{i}}$. After weighing, fresh enzyme solutions were added to the hydrogels. The experiments were performed in triplicate.

Human mesenchymal stem cells isolation: All polymers were sterilized by filtration of aqueous solutions through filters with a pore size of $0.22 \mu \mathrm{m}$ before lyophilization and thereafter stored in sterile tubes before use. Human bone marrow derived mesenchymal stem cells (hMSCs) were isolated as previously reported [37] and cultured in MSC proliferation medium ( $\alpha$-MEM (Gibco) supplemented with $10 \%$ fetal bovine serum, 1 \% L-glutamin (Gibco), $0.2 \mathrm{mM}$ ascorbic acid (Gibco), $100 \mathrm{U} / \mathrm{mL}$ penicillin, $10 \mu \mathrm{g} / \mathrm{mL}$ streptpmycin and $1 \mathrm{ng} / \mathrm{mL} \mathrm{bFGF}$ ). The use of human material was approved by a local medical ethical committee. Hydrogels containing hMSCs were prepared under sterile conditions by mixing a polymer/cell suspension together with $\mathrm{HRP}$ and $\mathrm{H}_{2} \mathrm{O}_{2}$ solutions (see section hydrogel formation and gelation time). PEG-TA $5-\mathrm{OH}_{3} / \mathrm{HA}-\mathrm{TA}_{2.2}$ solutions at different weight ratios $(100 / 0,80 / 20,50 / 50,20 / 80$ or $0 / 100)$ were prepared in proliferation medium and $\mathrm{HRP}$ and $\mathrm{H}_{2} \mathrm{O}_{2}$ stock solutions were made in PBS. Human mesenchymal stem cells (P3) were dispersed in the precursor solution of the hydrogels. The hydrogels were then formed as above mentioned in the absence of cells. The final polymer concentration was $10 \mathrm{wt} \%$ and the cell seeding density in the gels was $5 \times 10^{6}$ cells $/ \mathrm{mL}$. After gelation, the hydrogels (100 $\mu \mathrm{L}$ each) were transferred to a culture plate and $2 \mathrm{~mL}$ of hMSCs proliferation medium was added. The samples were incubated at $37{ }^{\circ} \mathrm{C}$ in a humidified atmosphere containing $5 \% \mathrm{CO}_{2}$. The medium was replaced every 3 or 4 days. 
Cell viability and proliferation. The metabolic activity and viability of hMSCs incorporated in the hydrogels were determined by a PrestoBlue assay and Live-dead assay according to the manufacturer's protocols [38]. At day 3, 7, 14, 21 and 28 the hydrogel constructs were rinsed with PBS and $30 \mu \mathrm{L}$ of PrestoBlue reagent in $270 \mu \mathrm{L}$ of hMSCs proliferation medium was added. Following an incubation time of 1 hour at 37 ${ }^{\circ} \mathrm{C}$, the PrestoBlue medium was transferred to a 96-well plate and the fluorescence of each well was measured using a microplate reader (Victor) with excitation at $560 \pm 10$ $\mathrm{nm}$ and emission at $590 \pm 10 \mathrm{~nm}$. Data are presented as average $\pm \mathrm{SD}(\mathrm{n}=3)$. Data were also statistically analyzed by using GraphPad Prism software. After removal of PrestoBlue medium, the hydrogel constructs were rinsed with PBS twice for $10 \mathrm{~min}$ each and then stained with calcein AM/ethidium homodimer using the Live-dead assay Kit (Invitrogen) according to the manufacturers' instructions. Hydrogel/cell constructs were visualized using a fluorescence microscope (EVOS). Living cells fluoresce green and the nuclei of dead cells red.

Chondrogenic differentiation of human chondrocytes in hydrogels: Human primary chondrocytes (hPCs) were obtained from full thickness cartilage dissected from knee biopsies of a patient undergoing total knee replacement as published previously [39] and cultured in chondrocyte expansion medium (DMEM with $10 \%$ heat inactivated fetal bovine serum, $1 \%$ penicillin/streptomycin (Gibco), $0.5 \mathrm{mg} / \mathrm{mL}$ fungizone (Gibco), 0.01 M MEM non-essential amino acids (Gibco) and $0.04 \mathrm{mM} \mathrm{L-proline)} \mathrm{at} 37{ }^{\circ} \mathrm{C}$ in a humidified atmosphere $\left(5 \% \mathrm{CO}_{2}\right)$. The use of human material was approved by a local medical ethical committee. Cylindrically shaped hydrogels with cells encapsulated were prepared using a home-made Teflon mold containing wells $(100 \mu \mathrm{L} /$ well, sterilized before use). PEG- $\mathrm{TA}_{5}-\mathrm{OH}_{3} / \mathrm{HA}-\mathrm{TA}_{2.2}$ hydrogel-cell constructs at different polymer weight ratios $(100 / 0,80 / 20,50 / 50,20 / 80$ or $0 / 100)$ were prepared by first mixing gel precursor solutions with chondrocytes (P3). The final polymer concentration was 12.5 wt $\%$. To each Teflon well $80 \mu \mathrm{L}$ of the mixture was added. Thereafter, a freshly prepared HRP solution ( $10 \mu \mathrm{L}$ of a $25 \mathrm{unit} / \mathrm{mL}$ stock solution) and $\mathrm{H}_{2} \mathrm{O}_{2}$ solution (10 $\mu \mathrm{L}, 0.3 \mathrm{wt} \%$ ) were mixed thoroughly, transferred to the wells and the mixture was quickly and gently shaken to allow gelation $(10 \mathrm{~s})$. The final cell density was $5 \times 10^{6}$ cells $/ \mathrm{mL}$ and the final concentration was $10 \mathrm{wt} \%$. All gel samples were then transferred to a 48 well plate and cultured in $1.0 \mathrm{~mL}$ of chondrogenic medium with transforming 
growth factor $\beta 3$ (TGF- $\beta 3$, R\&D System). Chondrogenic medium consisted of highglucose DMEM (Invitrogen), which was supplemented with $0.1 \mu \mathrm{M}$ dexamethasone, $1 \%$ penicillin/streptomycin, $100 \mu \mathrm{g} / \mathrm{mL}$ sodium pyruvate, $0.2 \mathrm{mM}$ L-ascorbic acid, 50 $\mathrm{mg} / \mathrm{mL}$ insulin-transferrin-selenite (ITS+1, Sigma) and $10 \mathrm{ng} / \mathrm{mL}$ TGF- $\beta 3$. Medium was changed every 3 or 4 days.

RNA isolation and real-time quantitative polymerase chain reaction (qPCR): After culturing the hydrogel/cell constructs in chondrogenic medium for 21 days, the samples were collected, washed with PBS and homogenized with a plastic grinding rod. Then 1 $\mathrm{mL}$ of Trizol reagent (Invitrogen, Carlsbad, CA) was added. Total RNA was isolated from the lysate according to the manufacturer's protocol [40]. The concentration of RNA was measured using the Nanodrop 2000 (Thermo scientific, Wilmington, USA). Subsequently, the RNA ( $1 \mu \mathrm{g})$ was reverse transcribed into single strand cDNA using the iScript Kit (BioRad) according to the manufacturer's recommendations. One microliter of each normalized cDNA sample was analyzed using the "SensiMix" SYBR \& Fluorescein kit" (Bioline) and a real-time PCR Cycler (Bio-Rad CFX96, Hercules, CA). The expression of collagen type II and aggrecan (Table 5.1) was analyzed and normalized to the expression of the housekeeping gene glyceraldehyde-3phosphate dehydrogenase (GAPDH). For each reaction a melting curve was generated to test primer dimer formation and non-specific priming.

Table 5.1 Polymerase chain reaction primers

\begin{tabular}{|c|c|c|}
\hline Primer & Direction & Sequence \\
\hline \multirow[t]{2}{*}{ Human aggrecan } & Forward & 5' AGGCAGCGTGATCCTTACC 3' \\
\hline & Reverse & 5' GGCCTCTCCAGTCTCATTCTC 3' \\
\hline \multirow{2}{*}{$\begin{array}{c}\text { Human collagen type } \\
\qquad \text { IIa1 }\end{array}$} & Forward & 5' CGTCCAGATGACCTTCCTACG 3' \\
\hline & Reverse & 5' TGAGCAGGGCCTTCTTGAG 3' \\
\hline \multirow[t]{2}{*}{ Human GAPDH } & Forward & 5' CGCTCTCTGCTCCTCCTGTT 3' \\
\hline & Reverse & 5' CCATGGTGTCTGAGCGATGT 3' \\
\hline
\end{tabular}

Histological analysis: Samples cultured for 28 days were fixed in $10 \%$ formalin for $2 \mathrm{~h}$ and then washed with PBS sucrose solution $(20 \%)$ for $2 \mathrm{~h}$ at $4{ }^{\circ} \mathrm{C}$. The samples were 
transferred to $1 \%$ PVA ( $\mathrm{Mw}=1 \mathrm{kDa})$ to reduce the formation of ice crystals when freezing for cryosectioning [41]. Constructs were then removed from the PVA solution, transferred to cryomolds with one flat plane facedown. Each cryomold was then filled with Cryomatrix $^{\mathrm{TM}}$ (Thermo Scientific) and frozen in liquid nitrogen. After solidification, cryomolds were removed from the slurry, stored at $-80{ }^{\circ} \mathrm{C}$ until cryosectioning. Routine cryosectioning was performed on the frozen blocks using a cryostat (Cryotome ${ }^{\mathrm{TM}} \mathrm{FE}$ ) at $-20{ }^{\circ} \mathrm{C}$. For each section, $10 \mu \mathrm{m}$ cross sections along the flat plane of the construct were collected on SuperFrost ${ }^{\mathbb{R}}$ Plus Gold slides (Fisher Scientific). The slides were air-dried for several hours before Alcian Blue staining and Safranin-O staining.

Alcian blue staining: Proteoglycan deposition and distribution was visualized by staining sections with Alcian Blue. Typically slides were stained for sulfated glycosaminoglycans (GAG) with a $0.5 \mathrm{wt} \%$ solution of Alcian blue $(\mathrm{pH}=1$, adjusted with $\mathrm{HCl})$ for $30 \mathrm{~min}$. The samples were then counterstained with nuclear fast red $(0.1$ $\mathrm{wt} \%$ of nuclear fast red in $5 \%$ aluminum sulfate) for $5 \mathrm{~min}$ to visualize nuclei. The slides were then washed with water and dehydrated.

Safranin-O staining: Safranin-O stains acidic proteoglycans present in cartilage tissues. Typically, slides were stained with a $0.1 \mathrm{wt} \%$ solution of Safranin-O for $5 \mathrm{~min}$, and counterstained with haematoxylin for $4 \mathrm{~min}$ to visualize nuclei. The slides were then washed with water and dehydrated. Sections were analyzed using a bright field microscope (Hamamatsu Nanozoomer).

Statistical analysis. Statistical differences between two groups were analyzed using a Student's $t$-test. Those among three or more groups were analyzed using the One-way Analysis of Variance (ANOVA) with Tukey's post-hoc analysis. Statistical significance was set to a $p$ value $\leq 0.01$. Results are presented as mean \pm standard deviation.

\subsection{Results and discussion}

\subsubsection{Synthesis of PEG-TA $-\mathrm{OH}_{3}$ and HA-TA 2.2}

The synthesis of partially tyramine functionalized 8 arm poly(ethylene glycol) (PEG$\left.(\mathrm{OH})_{8}\right)$ was performed by first reacting the hydroxyl groups of PEG- $(\mathrm{OH})_{8}$ with p- 
nitrophenyl chloroformate (PNC) to give $\mathrm{PEG}-\mathrm{PNC}_{5}-\mathrm{OH}_{3}$ as previously reported [42]. Attempting to reach higher end-group activation using a large excess of p-nitrophenyl chloroformate resulted in increasing amount of side products. Because the PEG-PNC $5^{-}$ $\mathrm{OH}_{3}$ could be obtained in high yield and purity no further attempts were made to increase the degree of the activation. Reaction of $\mathrm{PEG}-\mathrm{PNC}_{5}-\mathrm{OH}_{3}$ with a two times excess of tyramine (TA) afforded $\mathrm{PEG}-\mathrm{TA}_{5}-\mathrm{OH}_{3}$ as determined from the relative integrals of TA (6.79-6.99 ppm) and PEG peaks (3.63 ppm) (Figure 5.1). The signal at $4.2 \mathrm{ppm}$ represents the end $-\mathrm{CH}_{2}-\mathrm{O}-\mathrm{C}(\mathrm{O})$ - protons. The integral ratio of the tyramine aromatic protons and this signal was 5:2, conforming the average degree of end-group modification of 5 tyramine units per polymer molecule. The synthesis of tyramine conjugated hyaluronic acid $\left(\mathrm{HA}_{-} \mathrm{TA}_{2.2}\right)$ by EDAC/NHS activation of HA and reaction with the amine was previously reported [8].
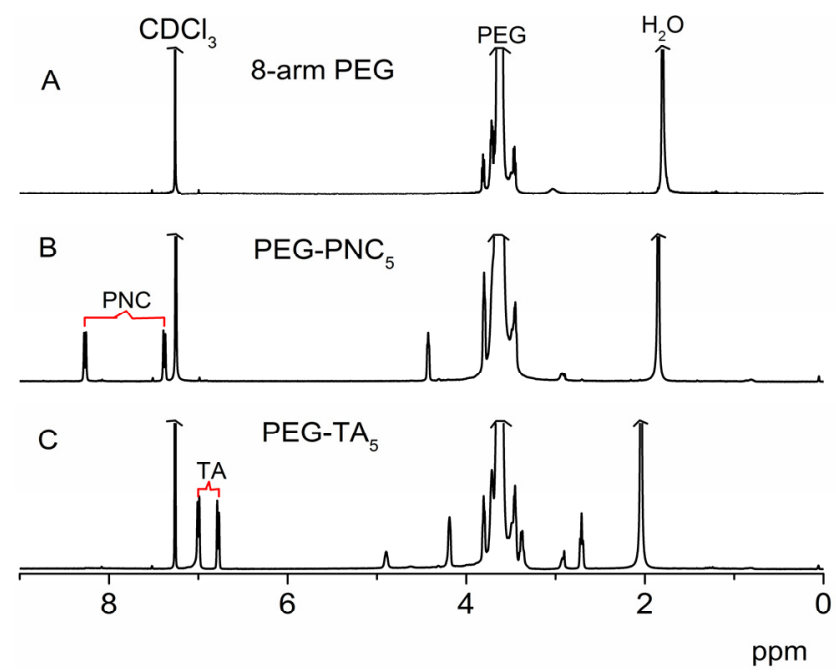

Figure 5.1 ${ }^{1} \mathrm{H}$ NMR spectra $\left(400 \mathrm{MHz}, \mathrm{CDCl}_{3}\right)$ of 8 arm PEG (A), PEG-PNC ${ }_{5}-\mathrm{OH}_{3}$ (B) and $\mathrm{PEG}-\mathrm{TA}_{5}-\mathrm{OH}_{3}(\mathrm{C})$.

\subsubsection{Hydrogel formation}

Enzymatic crosslinking of aqueous solutions of the tyramine conjugated polymers PEG$\mathrm{TA}_{5}-\mathrm{OH}_{3}, \mathrm{HA}-\mathrm{TA}_{2.2}$ and mixture thereof using horseradish peroxidase in the presence of hydrogen peroxide readily gave hydrogels. In previous research it was shown that at 
a HRP concentration of 15 units $/ \mathrm{mL}$ and $\mathrm{H}_{2} \mathrm{O}_{2}$ concentration of $0.01 \mathrm{M}$, hydrogels of dextran and hyaluronic acid were obtained within $1 \mathrm{~min}$ at concentrations ranging from 10 to $20 \mathrm{wt} \%$ [4]. Similar concentrations of $\mathrm{HRP}$ and $\mathrm{H}_{2} \mathrm{O}_{2}$ were used in initial gelation tests, however, instant gel formation was observed for this system. In order to accomplish suitable gelation times for the preparation of gel/cell constructs (vide infra) the concentration of HRP was decreased to $1 \sim 3$ units $/ \mathrm{mL}$ at a similar $\mathrm{H}_{2} \mathrm{O}_{2}$ concentration of $0.01 \mathrm{M}$. The gelation times of the polymer mixture PEG-TA5- $\mathrm{OH}_{3} / \mathrm{HA}$ $\mathrm{TA}_{2.2} 50 / 50$ decreased from $40 \mathrm{~s}$ to $10 \mathrm{~s}$ with an increasing concentration of HRP from 1 to 3 units $/ \mathrm{mL}$ and was independent of polymer concentration (Figure 5.2 a). Interestingly, at the highest polymer concentration, somewhat longer gelation times were observed ( $15 \mathrm{~s}$ vs $10 \mathrm{~s}, p<0.05)$, which may be due to the increased viscosity of solutions at higher polymer concentrations impairing mobility of the HRP. These initial experiments on the gelation kinetics of $\mathrm{PEG}-\mathrm{TA}_{5}-\mathrm{OH}_{3} / \mathrm{HA}-\mathrm{TA}_{2.2}$ revealed fast gelation even at a very low polymer concentration $(5 \mathrm{wt} \%)$ and 15 times lower HRP concentration (1 unit/mL) compared to Dex-TA/HA-TA gels as previously reported.

a

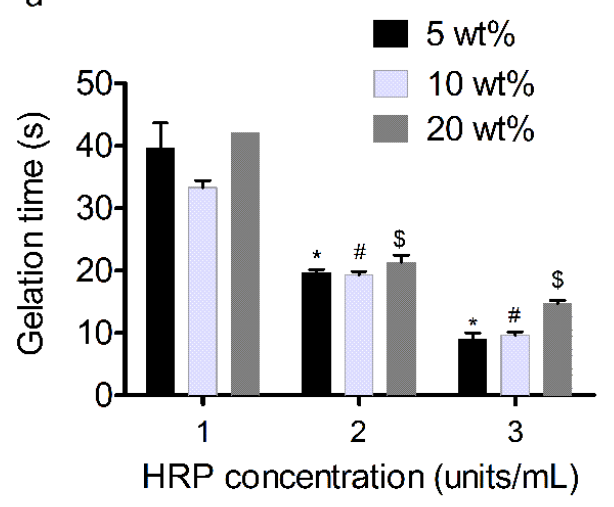

b

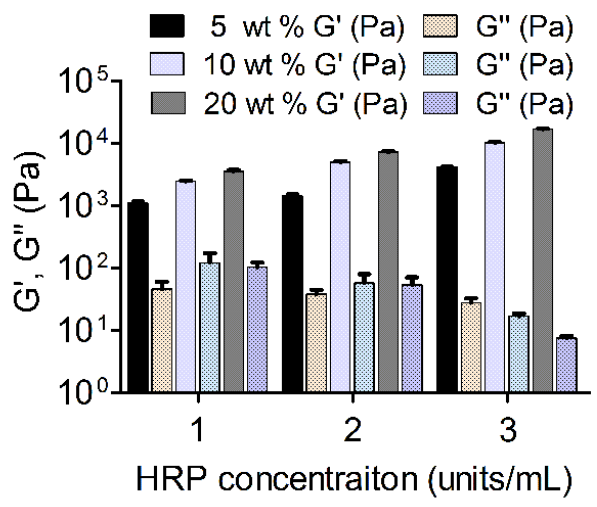

Figure 5.2 a: Gelation time of 5-20 wt\% PEG-TA $5-\mathrm{OH}_{3} / \mathrm{HA}-\mathrm{TA}_{2.2}(50 / 50)$ solutions as a function of HRP concentration. The $\mathrm{H}_{2} \mathrm{O}_{2}$ concentration was $0.01 \mathrm{M}$. $\left(\mathrm{n}=3,{ }^{*} p<0.05\right.$ vs $5 \mathrm{wt} \%, 1-3$ units $/ \mathrm{mL} ; \# p<0.05$ vs $10 \mathrm{wt} \%, 1-3$ units $/ \mathrm{mL} ; \$ p<0.05$ vs $20 \mathrm{wt} \%, 1-3$ units/mL.) b: the storage modulus (G') and loss modulus ( $\left.\mathrm{G}^{\prime \prime}\right)$ of 5 - $20 \mathrm{wt} \%$ PEG-TA $5^{-}$ $\mathrm{OH}_{3} / \mathrm{HA}-\mathrm{TA}_{2.2}(50 / 50)$ solutions as a function of HRP concentration. 


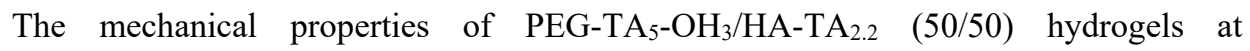
concentrations of 5,10 , and $20 \mathrm{wt} \%$ as a function of HRP concentration were studied by oscillatory rheology experiments at $25{ }^{\circ} \mathrm{C}$. Using a double syringe hydrogels were insitu formed at the plate of the rheometer and after lowering the measuring plate the storage and loss modulus were recorded. In Figure 5.2 b it is shown that at similar HRP concentrations, the storage modulus increased with increasing polymer concentration. At the same polymer concentration, the damping factor (G'/ $\left./ G^{\prime}\right)$ decreased with increasing HRP concentration. In following experiments, hydrogels were prepared at a HRP concentration of 2.5 units $/ \mathrm{mL}$

\subsubsection{Hydrogel properties}

The gel content and water uptake of $\mathrm{PEG}-\mathrm{TA}_{5}-\mathrm{OH}_{3} / \mathrm{HA}-\mathrm{TA}_{2.2}$ hydrogels with ratios ranging from $100 / 0$ to $0 / 100$ were subsequently determined. The variation in gel content and water uptake of $10 \mathrm{wt} \%$ hydrogels is presented in Figure 5.3. It was found that the gel content at all compositions was high ( $\geq 95 \%$ ), indicating that almost all polymers chains were incorporated into the networks. The water uptake increased from 13.3 to 16.3 with increasing HA-TA 2.2 weight ratios which can be attributed to a less efficient crosslinking by HRP due to increasing viscosity of the polymer mixture.

The kinetics of hydrogel formation was followed by monitoring the storage modulus $\left(G^{\prime}\right)$ and loss modulus (G') in time and results are presented in Figure 5.4 A. At all polymer ratios ( $\mathrm{PEG}-\mathrm{TA}_{5}-\mathrm{OH}_{3} / \mathrm{HA}-\mathrm{TA}_{2.2}$ ) no gelation point (defined as the crossover point of G' and G") was observed due to short gelation times. All hydrogels composed of PEG-TA $-\mathrm{OH}_{3} / \mathrm{HA}-\mathrm{TA}_{2.2}$ mixture showed high storage moduli, at least one order of magnitude higher than a hydrogel prepared from $\mathrm{HA}-\mathrm{TA}_{2.2}$ at the same $\mathrm{HRP}$ and $\mathrm{H}_{2} \mathrm{O}_{2}$ concentration. The storage moduli (G') and loss moduli (G') values graphically presented in Figure 5.4 B, revealed that highly elastic hydrogels were formed at different PEG- $\mathrm{TA}_{5}-\mathrm{OH}_{3} / \mathrm{HA}-\mathrm{TA}_{2.2}$ polymer ratios at very low enzyme and $\mathrm{H}_{2} \mathrm{O}_{2}$ concentrations. 


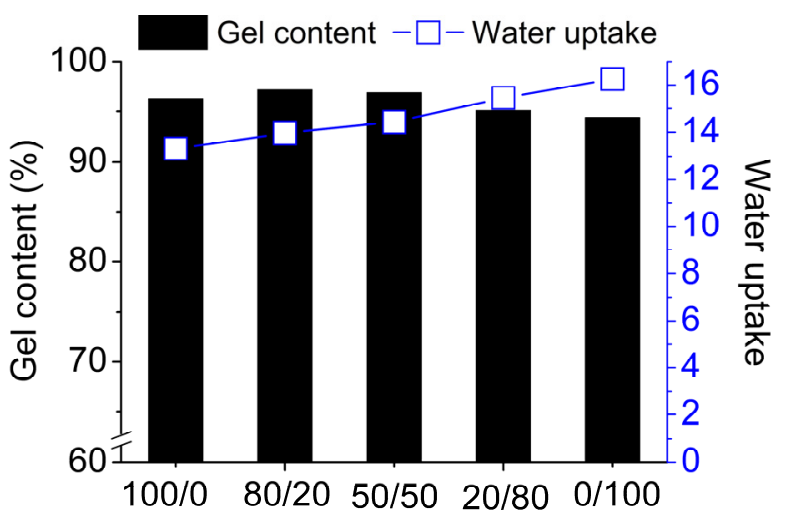

Figure 5.3 Gel content and water uptake of $10 \mathrm{wt} \%$ PEG-TA $-\mathrm{OH}_{3} / \mathrm{HA}-\mathrm{TA}_{2.2}$ hydrogels with various polymer compositions $(100 / 0$ to $0 / 100)$ at $10 \mathrm{wt} \%$.
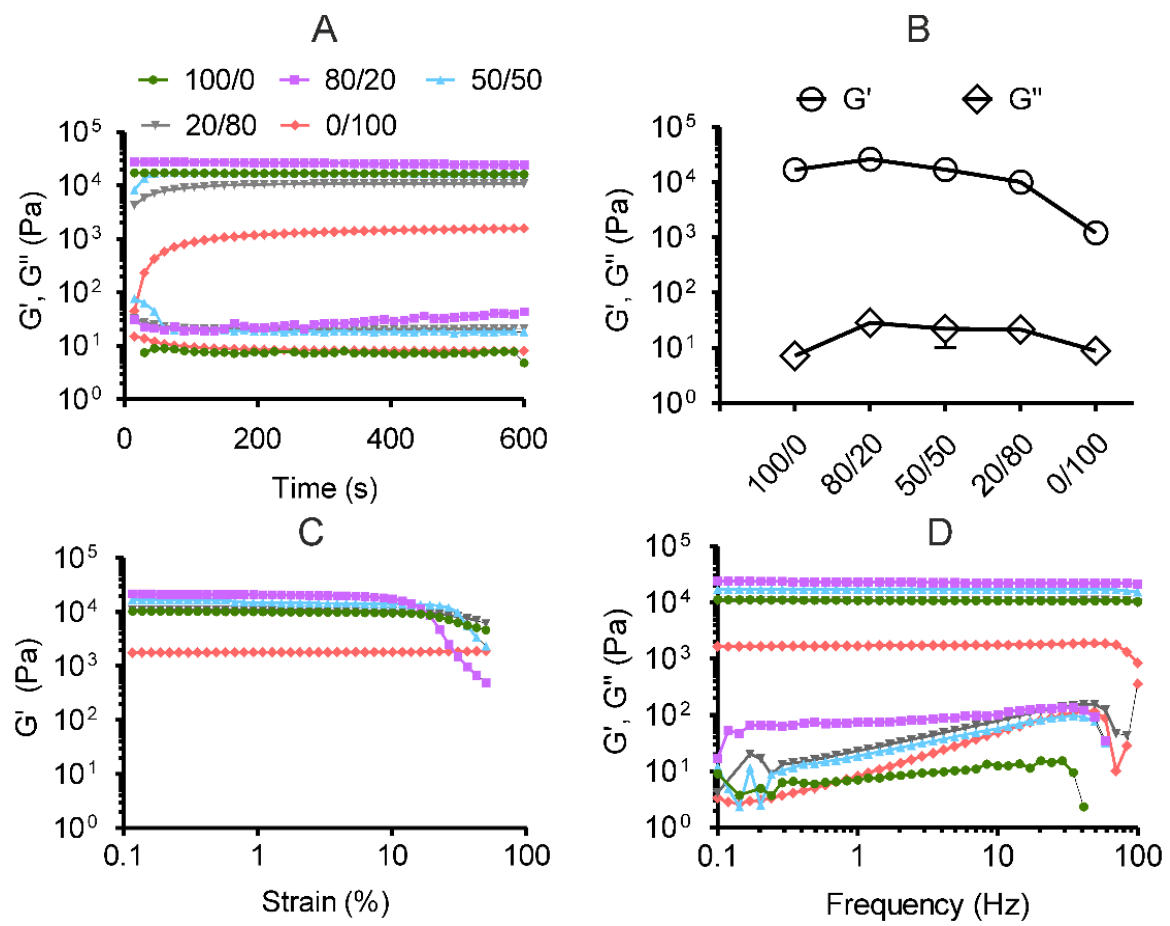

Figure 5.4 A: storage moduli (G', solid lines) and loss moduli (G', dotted lines) of 10 $\mathrm{wt} \%$ PEG-TA $-\mathrm{OH}_{3} / \mathrm{HA}-\mathrm{TA}_{2.2}$ hydrogels as a function of time; B: Strain sweep of $10 \mathrm{wt} \%$ PEG-TA $-\mathrm{OH}_{3} / \mathrm{HA}-\mathrm{TA}_{2.2}$ hydrogels; C: Frequency sweep of $10 \mathrm{wt} \%$ PEG-TA 5 $\mathrm{OH}_{3} / \mathrm{HA}-\mathrm{TA}_{2.2}$ hydrogels. Hydrogels were prepared at a $\mathrm{H}_{2} \mathrm{O}_{2}$ concentration of $0.01 \mathrm{M}$ and a HRP concentration of 2.5 units $/ \mathrm{mL}$. All measurements were performed at $25{ }^{\circ} \mathrm{C}$. 
Strain and frequency sweeps were subsequently performed. Using dynamic oscillation testing, an increasing cyclic strain ranging from 0.1 to $50 \%$ was applied at a frequency of $\omega_{0}=1.0 \mathrm{~Hz}$ (Figure 5.4 C). Hydrogels with higher PEG-TA $-\mathrm{OH}_{3}$ content preserve a constant high modulus up to $20 \%$ strain, but the modulus rapidly drops at higher strains. These results show that hydrogels comprising PEG-TA $-\mathrm{OH}_{3}$ are highly elastic but cannot resist large deformations. The magnitude of viscoelastic response elicited by a polymeric network is governed primarily by both the length and flexibility of the polymer chains [43-46]. Frequency sweeps from 0.1 to $100 \mathrm{~Hz}$ were conducted within the linear viscoelastic region at a strain amplitude $\gamma$ of $1 \%$. These measurements showed no decrease in the G' moduli of PEG-TA 5 /HA-TA 2.2 hydrogels and revealed a solid-like behavior over the entire frequency range (Figure 5.4 D).

\subsubsection{Compressive modulus}

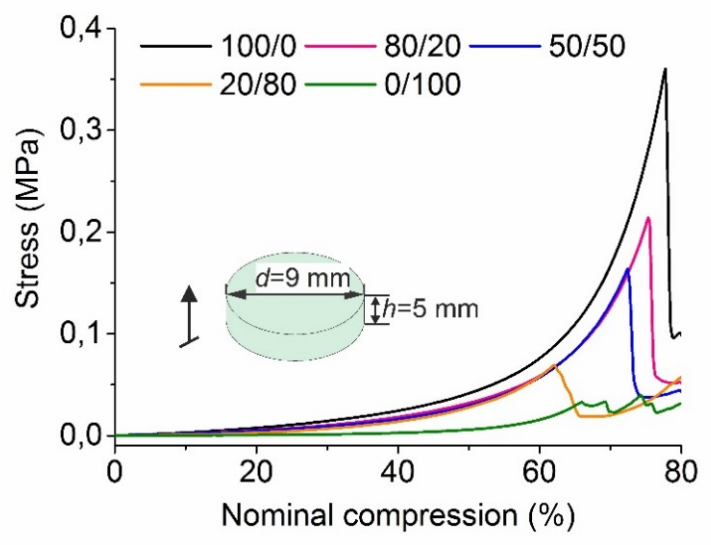

Figure 5.5 Compression tests of $10 \mathrm{wt} \%$ PEG-TA $5-\mathrm{OH}_{3} / \mathrm{HA}-\mathrm{TA}_{2.2}$ hydrogels at different ratios. $(n=5)$.

Because the stiffness has an important impact on cell function $[47,48]$, compression tests were used to examine the compressive modulus of these hydrogels from tissue engineering perspective. Hydrogels $(10 \mathrm{wt} \%$ ) containing various weight ratios of PEG$\mathrm{TA}_{5}-\mathrm{OH}_{3}$ and HA-TA 2.2 were pre-formed in PDMS molds and compressed up to failure. The stress-strain curves as depicted in Figure 5.5, revealed the highest modulus of the PEG-TA $5-\mathrm{OH}_{3}$ hydrogel at failure. Increasing the HA-TA 2.2 content the Young's 
modulus decreased and hydrogels yielded at lower stress and strain. The relation between storage modulus and Young's modulus was calculated from: $E=2 G^{\prime}(1+v)$, where $E$ is the Young's modulus, $G$ ' is the storage modulus and $v$ is Poisson's ratio. For rubber-like materials, the Poisson ratio (v) is assumed to be $\sim 0.5$ [49]. With the $G^{\prime}$ values obtained from rheological studies, the $E$ values of the hydrogels with different ratios are calculated ranging from $3.7-78.9 \mathrm{kPa}$.

\subsubsection{Hydrogel morphology}

The morphology and pore size of the hydrogels were determined by high resolution imaging. SEM images (Figure 5.6) of cross-sections of lyophilized hydrogels showed that the PEG-TA $-\mathrm{OH}_{3}$ hydrogel had a lower porosity and a more compact structure. This may due to very fast enzymatic crosslinking reaction; the gelation takes place immediately upon addition of HRP and $\mathrm{H}_{2} \mathrm{O}_{2}$. This hydrogel also showed the highest stiffness and lowest water uptake. A HA-TA $2.2(100 \%)$ gel had the highest pore size whereas at intermediate ratios the PEG-TA $5-\mathrm{OH}_{3} / \mathrm{HA}-\mathrm{TA}_{2.2}(80 / 20$ and 50/50) hydrogels showed intermediate porosities (Figure 5.6).

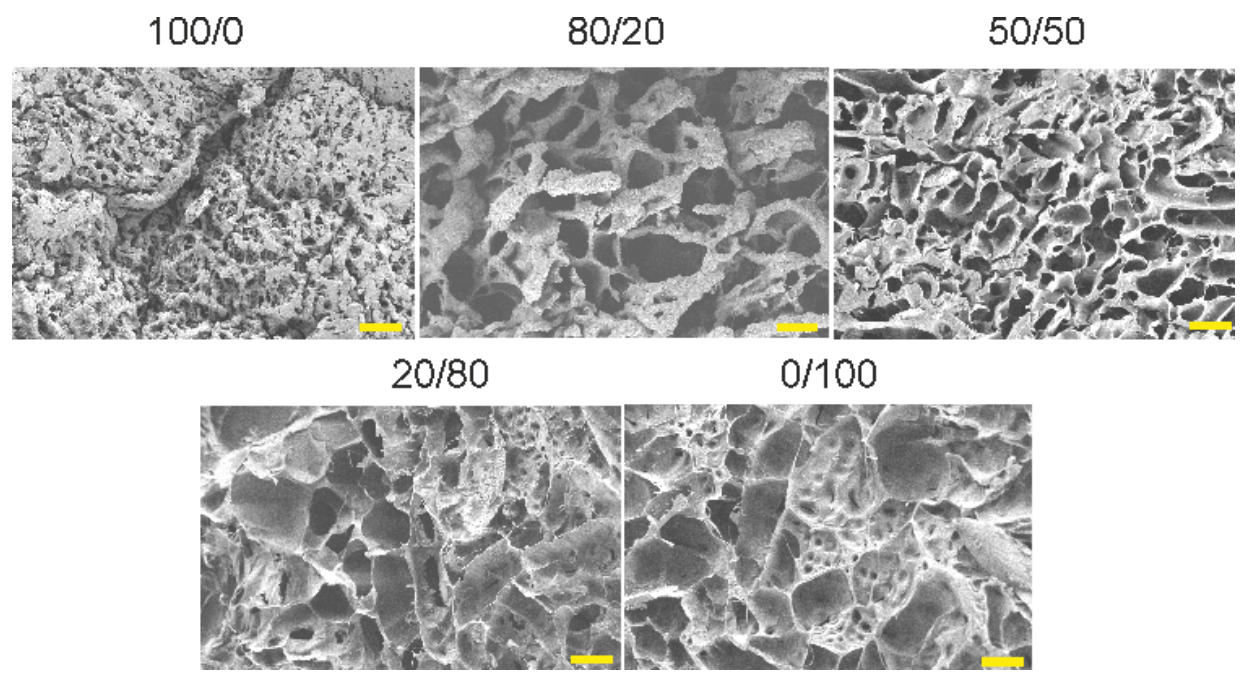

Figure 5.6 SEM images of $\mathrm{PEG}-\mathrm{TA}_{5}-\mathrm{OH}_{3} / \mathrm{HA}-\mathrm{TA}_{2.2}$ hydrogels at various weight ratios. Scale bar: $10 \mu \mathrm{m}$. 


\subsubsection{In vitro enzymatic degradation}

Degradation of scaffolds during tissue regeneration is a basis for remodeling and morphogenesis to form functional tissue. Hyaluronic acid can be degraded via enzymatic hydrolysis using hyaluronidase (HAse) [50]. The degradation of PEG-TA5$\mathrm{OH}_{3} / \mathrm{HA}-\mathrm{TA}_{2.2}$ hydrogels at different polymer ratios was determined by placing PBS containing $100 \mathrm{U} / \mathrm{mL}$ HAse on top of $0.2 \mathrm{~mL}$ hydrogels. The degradation at $37{ }^{\circ} \mathrm{C}$ was monitored by determining the remaining weight as a function of incubation time (Figure 5.7).

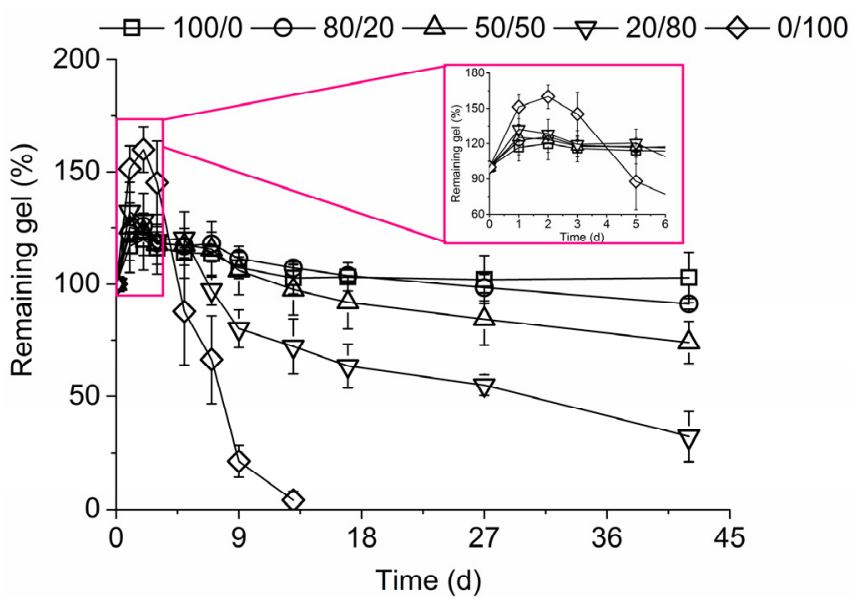

Figure 5.7 Enzymatic degradation of $10 \mathrm{wt} \%$ PEG-TA $5-\mathrm{OH}_{3} / \mathrm{HA}-\mathrm{TA}_{2.2}$ hydrogels in PBS containing $100 \mathrm{U} / \mathrm{mL}$ HAse at $37^{\circ} \mathrm{C}(\mathrm{n}=4)$.

The HA-TA 2.2 hydrogels showed the highest swelling (up to 165\%) in the first 3 days and were fully degraded within 2 weeks. Contrary, PEG-TA $5-\mathrm{OH}_{3}$ hydrogels did not show degradation over prolonged periods of time. Hydrogels composed of PEG-TA5$\mathrm{OH}_{3}$ and HA-TA 2.2 showed an intermediate degradation rate. After 6 weeks, the $10 \mathrm{wt} \%$ PEG-TA $5-\mathrm{OH}_{3} / \mathrm{HA}-\mathrm{TA}_{2.2}$ hydrogels with weight ratios of 20/80, 50/50 and 80/20 was 30, 75 and $96 \%$, respectively. Control experiments of gels in buffer without enzymes were carried out simultaneously showing similar swelling during the first 3 days, but no degradation over a $42 \mathrm{~d}$ period (data not shown). 


\subsubsection{Cytotoxicity and morphology}

In situ forming hydrogels allow an easy encapsulation of cells in the gel matrix. Human mesenchymal stem cells (hMSCs) in proliferation medium were mixed with a hydrogel precursor solution containing the enzyme HRP. These mixtures were subsequently mixed with a hydrogen peroxide solution to give hydrogels with a final polymer concentration of $10 \mathrm{wt} \%$. These hydrogel/cell constructs were analyzed for their cell cytotoxicity by a PrestoBlue assay and a live-dead assay for up to 28 days. The PB assay as presented in Figure 5.8, revealed that cells encapsulated in hydrogel constructs of different composition were metabolic active over the entire period. The HA-TA 2.2 hydrogels were degraded after 10 days in culture, therefore, no data was shown afterwards. The biocompatibility of these hydrogels was analyzed by a live-dead assay after culturing for 3, 7, 14 and 21 days, in which the live cells fluoresce green, and dead cells fluoresce red. Merged images with both green and red showed that over $95 \%$ of the cells fluoresced green after cultured 21 days, indicating cytocompatible enzymatic crosslinking conditions (Figure 5.9).

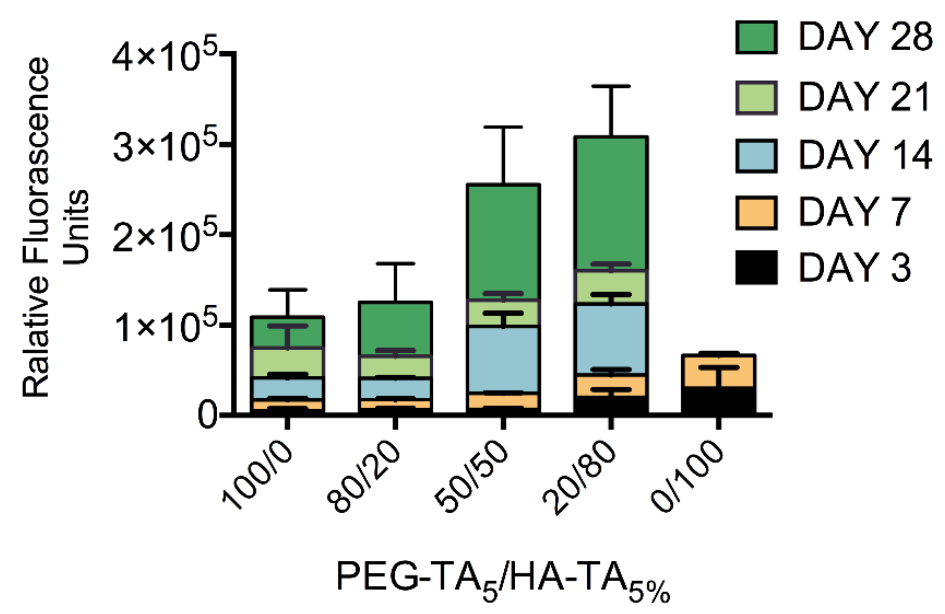

Figure 5.8 Metabolic activity of hMSCs encapsulated in PEG-TA5- $\mathrm{OH}_{3} / \mathrm{HA}-\mathrm{TA}_{2.2}$ hydrogels with weight ratios of $100 / 0$ to $0 / 100 \%$ at a concentration of $10 \mathrm{wt} \%$ (PrestoBlue assay). Cell seeding density: $5 \times 10^{6}$ cells $/ \mathrm{mL}$. 


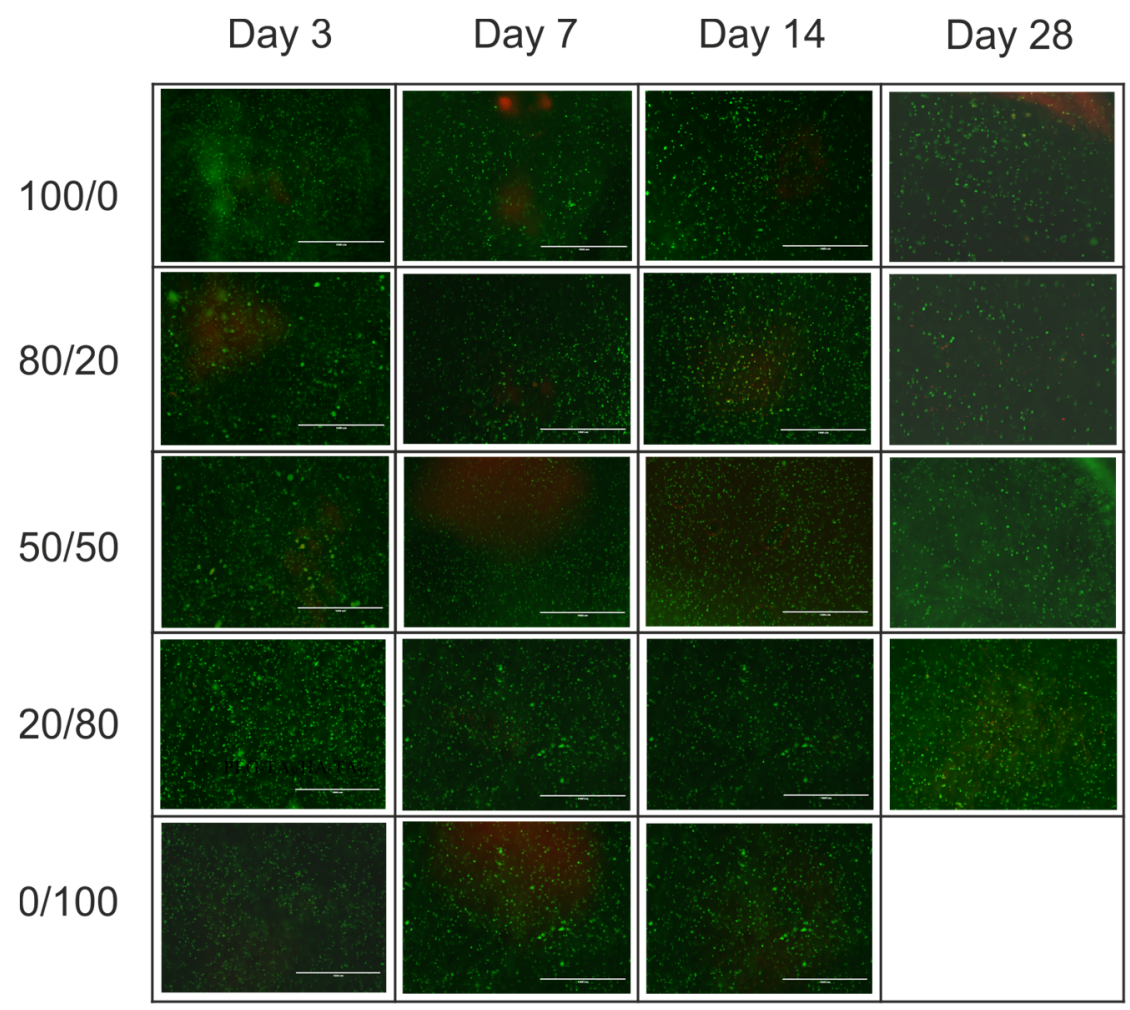

Figure 5.9 Live-dead assay showing hMSCs incorporated in PEG-TA $5-\mathrm{OH}_{3} / \mathrm{HA}-\mathrm{TA}_{2.2}$ hydrogels with weight ratios of $100 / 0$ to $0 / 100$ at a concentration of $10 \mathrm{wt} \%$ after 3,7 , 14 and 21 days in culture. Cell seeding density: $5 \times 10^{6}$ cells $/ \mathrm{mL}$. Scale bar: $1000 \mu \mathrm{m}$.

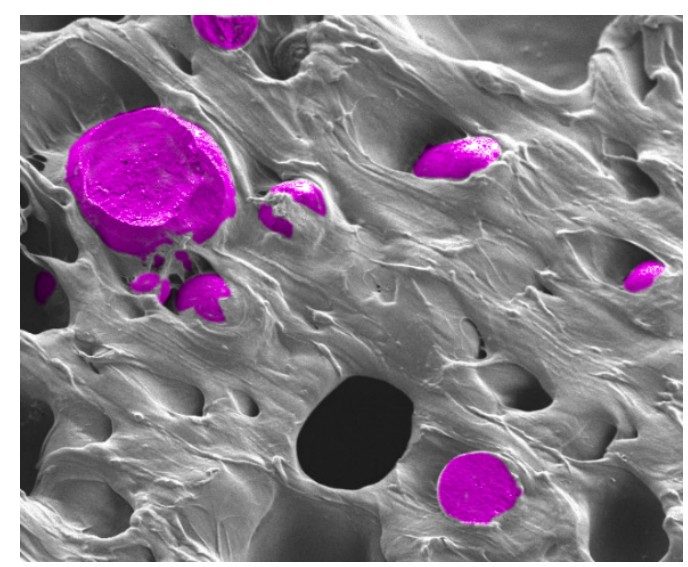

Figure 5.10 SEM image of hMSCs incorporated in a PEG-TA $-\mathrm{OH}_{3} / \mathrm{HA}-\mathrm{TA}_{2.2}$ 50/50 hydrogel (10 wt \%) at day 28 . Scale bar: $10 \mu \mathrm{m}$. 
A typical SEM image of a hydrogel cross-section of encapsulated hMSCs in a PEG$\mathrm{TA}_{5}-\mathrm{OH}_{3} / \mathrm{HA}-\mathrm{TA}_{2.2} 50 / 50$ at day 28 is shown in Figure 5.10. The cells encapsulated inside the gel retained a round shape for 28 days in culture, which indicates that the hydrogels were biocompatible and support cell survival but do not stimulate cell spreading.

\subsubsection{In vitro differentiation of human chondrocytes encapsulated in hydrogels}

Differentiation of human chondrocytes was quantitatively evaluated using RT-PCR. The ability of chondrocytes encapsulated in the hydrogels, to maintain a chondrocytic phenotype and form cartilaginous tissue in vitro in chondrogenic differentiation medium was followed up to 21 days. Gene expression of SOX 9, collagen type II and aggrecan as specific chondrogenic markers was analyzed and results are presented in Figure 5.11. The gene expression was normalized to control samples in which cells were cultured on tissue culture plastic (2D). As shown in Figure 5.11, chondrocytes in hydrogels (3D) expressed significantly higher levels of the markers compared to cells cultured in 2D, similar as reported by Discher et al. and Yang et al. [48,51].

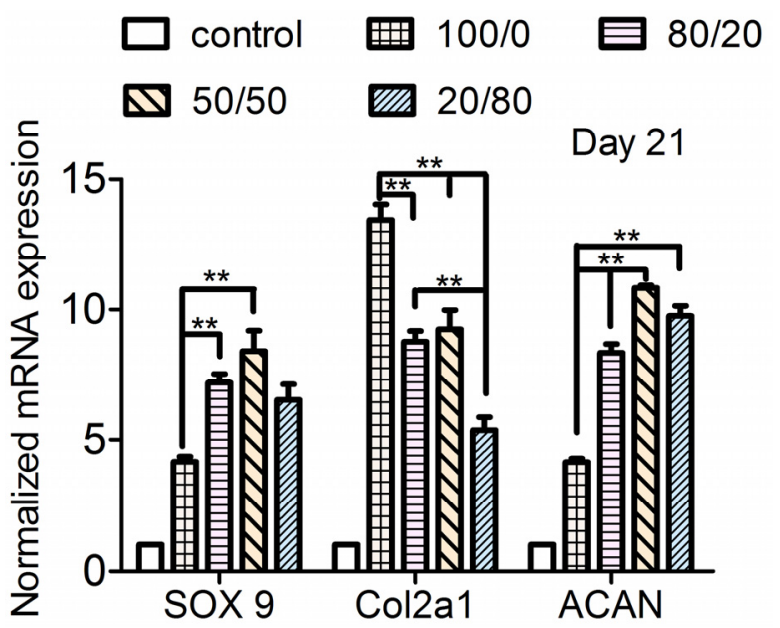

Figure 5.11 Gene expression profile of differentiating human chondrocytes at day 21 in culture. Expression of the specific cartilage makers Sox 9, Col2al and ACAN in $10 \mathrm{wt} \%$ 
PEG-TA5 $-\mathrm{OH}_{3} / \mathrm{HA}-\mathrm{TA}_{2.2}$ hydrogel $(100 / 0$ - 20/80) constructs are presented as fold change relative to mRNA expression from a control (cells cultured on tissue plate). Data are shown as mean $\pm \operatorname{SD}(\mathrm{n}=3)$.

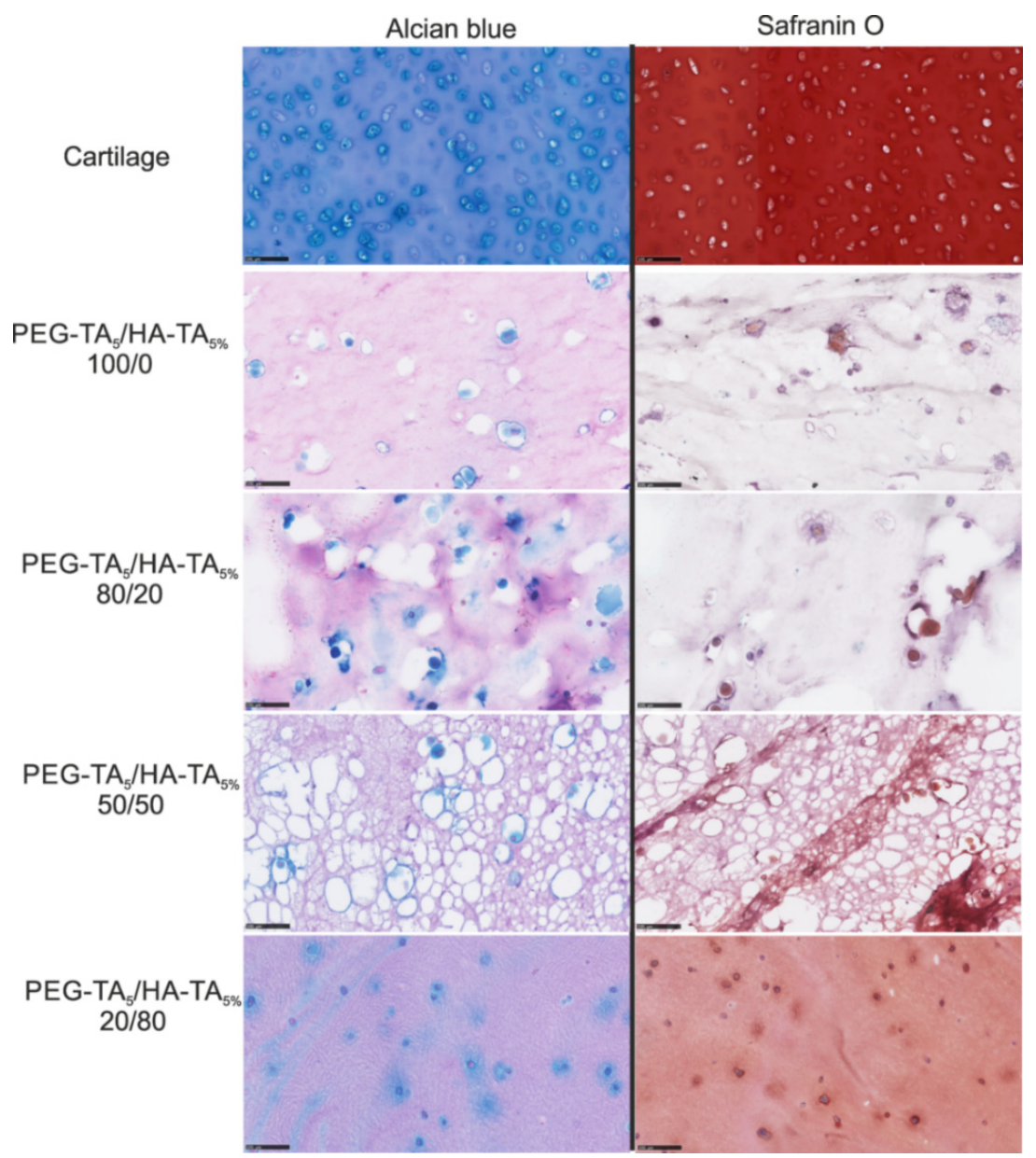

Figure 5.12 Alcian blue and safranin $\mathrm{O}$ staining of $\mathrm{PEG}-\mathrm{TA}_{5}-\mathrm{OH}_{3} / \mathrm{HA}-\mathrm{TA}_{2.2}$ hydrogels with weight ratios of 100/0 to 0/100 after culturing for 21 days. Scale bar: $100 \mu \mathrm{m}$.

The expression levels of these three genes depended on the hydrogel composition. Chondrocytes encapsulated in $\mathrm{PEG}-\mathrm{TA}_{5}-\mathrm{OH}_{3} / \mathrm{HA}-\mathrm{TA}_{2.2}$ 50/50 hydrogels showed significantly upregulated SOX 9 and ACAN gene expressions compared to those in PEG-TA5 $-\mathrm{OH}_{3}$ hydrogels $(p<0.01)$. However, PEG-TA $-\mathrm{OH}_{3}$ hydrogels expressed higher collagen type II levels than all other hydrogels, indicating an existence of a 
chondrocyte phenotype. The results showed that at higher HA content SOX9 and ACAN levels were upregulated in an inverse relation with the mechanical strength of the hydrogel. Our data also suggest a direct correlation between collagen type II mRNA expression levels and gel mechanical properties. An increased expression of Collagen type II is observed for hydrogels with higher storage modulus [48]. Additionally, the accumulation of a chondrogenic specific ECM in the hydrogel/cell constructs was examined by histological analysis. Bovine cartilage sections were stained as positive control. Alcian blue and Safranin O, which stain glycosaminoglycan with an intense blue color (Figure 5.12, left) and acidic proteoglycans with an orange-red color (Figure 5.12, right) were clearly observed in hydrogels after 28 days in culture, showing the production of a cartilage specific ECM. As shown in Figure 5.12, GAG and proteoglycan stainings were observed in all hydrogels with different compositions. PEG- $\mathrm{TA}_{5}-\mathrm{OH}_{3}$ hydrogels showed distinct cell encapsulation and less ECM production, which may be due to the high stiffness of the PEG gels and low cell-hydrogel interactions [52]. However, in the presence of HA-TA2.2, intense blue and red were produced. Especially, in the PEG-TA $5-\mathrm{OH}_{3} / \mathrm{HA}-\mathrm{TA}_{2.2} 20 / 80$ hydrogel, newly formed matrix was evenly distributed over the construct. This may be due to HA which can easily interact with proteins present on the surface of the cells as well as with cells via CD44 cell surface receptors.

\subsection{Conclusions}

Biodegradable synthetic/natural hybrid hydrogels were successfully formed by the oxidative coupling reaction of tyramine conjugates of a star shaped 8 arm PEG and hyaluronic acid in the presence of $\mathrm{H}_{2} \mathrm{O}_{2}$ and low HRP. The physicochemical properties of the hydrogels, including gelation time, mechanical properties, swelling ratio and degradation could be adjusted by varying the HRP concentration and polymer composition. Hybrid hydrogels with different ratios of $8 \mathrm{PEG}$ and HA showed excellent cytocompatibitliy. The metabolic activity of hMSCs encapsulated in the hydrogels was highest at a higher HA content of $80 \%$. Moreover, human chondrocytes encapsulated in hydrogels maintained their chondrocyte phenotype and formed a cartilaginous specific matrix. High collagen type II expression was observed in higher elastic gels. 
Interestingly, higher SOX9 and ACAN expression was present in gels with higher HA content resulting in gels with lower stiffness. These results demonstrate that PEGTA/HA-TA hybrid hydrogels are promising candidates for tissue regeneration applications.

\section{Reference}

[1] Jin, R.; Hiemstra, C.; Zhong, Z. Y.; Feijen, J., Enzyme-mediated fast in situ formation of hydrogels from dextran-tyramine conjugates. Biomaterials 2007, 28 (18), 2791-2800.

[2] Lee, F.; Chung, J. E.; Kurisawa, M., An injectable enzymatically crosslinked hyaluronic acid-tyramine hydrogel system with independent tuning of mechanical strength and gelation rate. Soft Matter 2008, 4 (4), 880-887.

[3] Lee, F.; Chung, J. E.; Kurisawa, M., An injectable hyaluronic acid-tyramine hydrogel system for protein delivery. J. Controlled Release 2009, 134 (3), 186-193.

[4] Wennink, J. W. H.; Niederer, K.; Bochynska, A. I.; Teixeira, L. S. M.; Karperien, M.; Feijen, J.; Dijkstra, P. J., Injectable Hydrogels by Enzymatic Co-Crosslinking of Dextran and Hyaluronic Acid Tyramine Conjugates. Advanced Polymers in Medicine 2011, 309-310 (1), 213-221.

[5] Teixeira, L. S. M.; Bijl, S.; Pully, V. V.; Otto, C.; Rong, J.; Feijen, J.; van Blitterswijk, C. A.; Dijkstra, P. J.; Karperien, M., Self-attaching and cell-attracting insitu forming dextran-tyramine conjugates hydrogels for arthroscopic cartilage repair. Biomaterials 2012, 33 (11), 3164-3174.

[6] Xu, K. M.; Lee, F.; Gao, S. J.; Chung, J. E.; Yano, H.; Kurisawa, M., Injectable hyaluronic acid-tyramine hydrogels incorporating interferon-alpha $2 \mathrm{a}$ for liver cancer therapy. J. Controlled Release 2013, 166 (3), 203-210.

[7] Wang, L. S.; Lee, F.; Lim, J.; Du, C.; Wan, A. C. A.; Lee, S. S.; Kurisawa, M., Enzymatic conjugation of a bioactive peptide into an injectable hyaluronic acidtyramine hydrogel system to promote the formation of functional vasculature. Acta Biomater. 2014, 10 (6), 2539-2550. 
[8] Wang, R.; Leber, N.; Buhl, C.; Verdonschot, N.; Dijkstra, P. J.; Karperien, M., Cartilage adhesive and mechanical properties of enzymatically crosslinked polysaccharide tyramine conjugate hydrogels. Polym. Adv. Technol. 2014, 25 (5), 568574.

[9] Gohil, S. V.; Brittain, S. B.; Kan, H.-M.; Drissi, H.; Rowe, D. W.; Nair, L. S., Evaluation of enzymatically crosslinked injectable glycol chitosan hydrogel. J. Phys. Chem. B 2015, 3 (27), 5511-5522.

[10] Sakai, S.; Khanmohammadi, M.; Khoshfetrat, A. B.; Taya, M., Horseradish peroxidase-catalyzed formation of hydrogels from chitosan and poly(vinyl alcohol) derivatives both possessing phenolic hydroxyl groups. Carbohydr. Polym. 2014, 111 (404-409.

[11] Li, L.; Wang, N.; Jin, X.; Deng, R.; Nie, S.; Sun, L.; Wu, Q.; Wei, Y.; Gong, C., Biodegradable and injectable in situ cross-linking chitosan-hyaluronic acid based hydrogels for postoperative adhesion prevention. Biomaterials 2014, 35 (12), 39033917.

[12] Tran, N. Q.; Joung, Y. K.; Lih, E.; Park, K. D., In Situ Forming and RutinReleasing Chitosan Hydrogels As Injectable Dressings for Dermal Wound Healing. Biomacromolecules 2011, 12 (8), 2872-2880.

[13] Nair, S.; Remya, N. S.; Remya, S.; Nair, P. D., A biodegradable in situ injectable hydrogel based on chitosan and oxidized hyaluronic acid for tissue engineering applications. Carbohydr. Polym. 2011, 85 (4), 838-844.

[14] Lih, E.; Joung, Y. K.; Bae, J. W.; Park, K. D., An in situ gel-forming heparinconjugated PLGA-PEG-PLGA copolymer. J. Bioact. Compatible Polym. 2008, 23 (5), 444-457.

[15] Su, T.; Zhang, D.; Tang, Z.; Wu, Q.; Wang, Q. G., HRP-mediated polymerization forms tough nanocomposite hydrogels with high biocatalytic performance. Chem. Commun. 2013, 49 (73), 8033-8035.

[16] Jin, R.; Moreira Teixeira, L. S.; Dijkstra, P. J.; Zhong, Z.; van Blitterswijk, C. A.; Karperien, M.; Feijen, J., Enzymatically crosslinked dextran-tyramine hydrogels as injectable scaffolds for cartilage tissue engineering. Tissue Eng Part A 2010, 16 (8), 2429-2440. 
[17] Sofia, S. J.; Singh, A.; Kaplan, D. L., Peroxidase-catalyzed crosslinking of functionalized polyaspartic acid polymers. J. Macromol sci., Part A 2002, 39 (10), 1151-1181.

[18] Hou, J. X.; Li, C.; Guan, Y.; Zhang, Y. J.; Zhu, X. X., Enzymatically crosslinked alginate hydrogels with improved adhesion properties. Polym Chem-Uk 2015, 6 (12), 2204-2213.

[19] Sakai, S.; Kawakami, K., Development of Porous Alginate-Based Scaffolds Covalently Cross-Linked through a Peroxidase-Catalyzed Reaction. J. Biomater. Sci. Polym. Ed. 2011, 22 (18), 2407-2416.

[20] Sakai, S.; Hirose, K.; Moriyama, K.; Kawakami, K., Control of cellular adhesiveness in an alginate-based hydrogel by varying peroxidase and $\mathrm{H} 2 \mathrm{O} 2$ concentrations during gelation. Acta Biomater. 2010, 6 (4), 1446-1452.

[21] Lee, Y.; Bae, J. W.; Oh, D. H.; Park, K. M.; Chun, Y. W.; Sung, H. J.; Park, K. D., In situ forming gelatin-based tissue adhesives and their phenolic content-driven properties. J. Phys. Chem. B 2013, 1 (18), 2407-2414.

[22] Kondo, D.; Ogino, Y.; Ayukawa, Y.; Sakai, S.; Kawakami, K.; Koyano, K., Bone Regeneration of Tibial Defects in Rats with Enzymatic Hydrogelation of Gelatin Derivative and Recombinant Human Platelet-Derived Growth Factor-BB Complex. Int. J. Oral Maxillofac. Implants 2013, 28 (5), 1377-1385.

[23] Wang, L. S.; Du, C.; Chung, J. E.; Kurisawa, M., Enzymatically cross-linked gelatin-phenol hydrogels with a broader stiffness range for osteogenic differentiation of human mesenchymal stem cells. Acta Biomater. 2012, 8 (5), 1826-1837.

[24] Park, K. M.; Lee, Y.; Son, J. Y.; Oh, D. H.; Lee, J. S.; Park, K. D., Synthesis and Characterizations of In Situ Cross-Linkable Gelatin and 4-Arm-PPO-PEO Hybrid Hydrogels via Enzymatic Reaction for Tissue Regenerative Medicine. Biomacromolecules 2012, 13 (3), 604-611.

[25] Park, K. M.; Lee, Y.; Son, J. Y.; Bae, J. W.; Park, K. D., In Situ SVVYGLR Peptide Conjugation into Injectable Gelatin-Poly(ethylene glycol)-Tyramine Hydrogel via Enzyme-Mediated Reaction for Enhancement of Endothelial Cell Activity and NeoVascularization. Bioconjugate Chem. 2012, 23 (10), 2042-2050. 
[26] Park, K. M.; Ko, K. S.; Joung, Y. K.; Shin, H.; Park, K. D., In situ cross-linkable gelatin-poly(ethylene glycol)-tyramine hydrogel via enzyme-mediated reaction for tissue regenerative medicine. J. Mater. Chem. 2011, 21 (35), 13180-13187.

[27] Wang, L. S.; Boulaire, J.; Chan, P. P. Y.; Chung, J. E.; Kurisawa, M., The role of stiffness of gelatin-hydroxyphenylpropionic acid hydrogels formed by enzymemediated crosslinking on the differentiation of human mesenchymal stem cell. Biomaterials 2010, 31 (33), 8608-8616.

[28] Sakai, S.; Tsumura, M.; Inoue, M.; Koga, Y.; Fukano, K.; Taya, M., Polyvinyl alcohol-based hydrogel dressing gellable on-wound via a co-enzymatic reaction triggered by glucose in the wound exudate. J. Phys. Chem. B 2013, 1 (38), 5067-5075.

[29] Park, K. M.; Jun, I.; Joung, Y. K.; Shin, H.; Park, K. D., In situ hydrogelation and RGD conjugation of tyramine-conjugated 4-arm PPO-PEO block copolymer for injectable bio-mimetic scaffolds. Soft Matter 2011, 7 (3), 986-992.

[30] Park, K. M.; Shin, Y. M.; Joung, Y. K.; Shin, H.; Park, K. D., In Situ Forming Hydrogels Based on Tyramine Conjugated 4-Arm-PPO-PEO via Enzymatic Oxidative Reaction. Biomacromolecules 2010, 11 (3), 706-712.

[31] Li, J.; Ni, X.; Leong, K. W., Injectable drug-delivery systems based on supramolecular hydrogels formed by poly(ethylene oxide)s and alpha-cyclodextrin. $J$. Biomed. Mater. Res. A 2003, 65 (2), 196-202.

[32] Wu, C. Z.; Strehmel, C.; Achazi, K.; Chiapisi, L.; Dernedde, J.; Lensen, M. C.; Gradzielski, M.; Ansorge-Schumacher, M. B.; Haag, R., Enzymatically Cross-Linked Hyperbranched Polyglycerol Hydrogels as Scaffolds for Living Cells. Biomacromolecules 2014, 15 (11), 3881-3890.

[33] Seidlits, S. K.; Khaing, Z. Z.; Petersen, R. R.; Nickels, J. D.; Vanscoy, J. E.; Shear, J. B.; Schmidt, C. E., The effects of hyaluronic acid hydrogels with tunable mechanical properties on neural progenitor cell differentiation. Biomaterials 2010, 31 (14), 39303940.

[34] Kogan, G.; Soltes, L.; Stern, R.; Gemeiner, P., Hyaluronic acid: a natural biopolymer with a broad range of biomedical and industrial applications. Biotechnol. Lett 2007, 29 (1), 17-25.

[35] Jiang, D.; Liang, J.; Noble, P. W. In Annu. Rev. Cell Dev. Biol. 2007; Vol. 23, p 435-461. 
[36] Wang, R.; Geven, M.; Dijkstra, P. J.; Martens, P.; Karperien, M., Hydrogels by supramolecular crosslinking of terpyridine end group functionalized 8-arm poly(ethylene glycol). Soft Matter 2014, 10 (37), 7328-7336.

[37] Fernandes, H.; Dechering, K.; Van Someren, E.; Steeghs, I.; Apotheker, M.; Leusink, A.; Bank, R.; Janeczek, K.; Van Blitterswijk, C.; de Boer, J., The role of collagen crosslinking in differentiation of human mesenchymal stem cells and MC3T3E1 cells. Tissue Eng. Part A 2009, 15 (12), 3857-3867.

[38] Boncler, M.; Rozalski, M.; Krajewska, U.; Podsedek, A.; Watala, C., Comparison of PrestoBlue and MTT assays of cellular viability in the assessment of antiproliferative effects of plant extracts on human endothelial cells. J. Pharmacol. Toxicol. Methods 2014, 69 (1), 9-16.

[39] Hendriks, J. A. A.; Miclea, R. L.; Schotel, R.; de Bruijn, E.; Moroni, L.; Karperien, M.; Riesle, J.; van Blitterswijk, C. A., Primary chondrocytes enhance cartilage tissue formation upon co-culture with a range of cell types. Soft Matter 2010, 6 (20), 50805088.

[40] Hummon, A. B.; Lim, S. R.; Difilippantonio, M. J.; Ried, T., Isolation and solubilization of proteins after TRIzol((R)) extraction of RNA and DNA from patient material following prolonged storage. BioTechniques 2007, 42 (4), 467-472.

[41] Ruan, J. L.; Tulloch, N. L.; Muskheli, V.; Genova, E. E.; Mariner, P. D.; Anseth, K. S.; Murry, C. E., An improved cryosection method for polyethylene glycol hydrogels used in tissue engineering. Tissue Eng Part C Methods 2013, 19 (10), 794-801.

[42] Wang, R.; Both, S. K.; Geven, M.; Calucci, L.; Forte, C.; Dijkstra, P. J.; Karperien, M., Kinetically stable metal ligand charge transfer complexes as crosslinks in nanogels/hydrogels: Physical properties and cytotoxicity. Acta Biomater. 2015, 26 (136144.

[43] Kloxin, A. M.; Kloxin, C. J.; Bowman, C. N.; Anseth, K. S., Mechanical Properties of Cellularly Responsive Hydrogels and Their Experimental Determination. Adv. Mater. 2010, 22 (31), 3484-3494.

[44] Anseth, K. S.; Bowman, C. N.; BrannonPeppas, L., Mechanical properties of hydrogels and their experimental determination. Biomaterials 1996, 17 (17), 1647-1657. 
[45] Teng, D.-y.; Wu, Z.-m.; Zhang, X.-g.; Wang, Y.-x.; Zheng, C.; Wang, Z.; Li, C.-X., Synthesis and characterization of in situ cross-linked hydrogel based on self-assembly of thiol-modified chitosan with PEG diacrylate using Michael type addition. Polymer 2010, 51 (3), 639-646.

[46] Kavanagh, G. M.; Ross-Murphy, S. B., Rheological characterisation of polymer gels. Prog. Polym. Sci. 1998, 23 (3), 533-562.

[47] Discher, D. E.; Mooney, D. J.; Zandstra, P. W., Growth Factors, Matrices, and Forces Combine and Control Stem Cells. Science 2009, 324 (5935), 1673-1677.

[48] Engler, A. J.; Sen, S.; Sweeney, H. L.; Discher, D. E., Matrix elasticity directs stem cell lineage specification. Cell 2006, 126 (4), 677-689.

[49] W., M. C. Rheology: principles, measurements and applications; Wiley-VCH, 1994; Vol. Ch.1.

[50] Menzel, E. J.; Farr, C., Hyaluronidase and its substrate hyaluronan: biochemistry, biological activities and therapeutic uses. Cancer Lett. 1998, 131 (1), 3-11.

[51] Liu, S. Q.; Tian, Q.; Hedrick, J. L.; Hui, J. H. P.; Ee, P. L. R.; Yang, Y. Y., Biomimetic hydrogels for chondrogenic differentiation of human mesenchymal stem cells to neocartilage. Biomaterials 2010, 31 (28), 7298-7307.

[52] Discher, D. E.; Janmey, P.; Wang, Y. L., Tissue cells feel and respond to the stiffness of their substrate. Science 2005, 310 (5751), 1139-1143. 


\section{Chapter 6}

\section{Hydrogels by supramolecular crosslinking of terpyridine end group functionalized 8 arm poly(ethylene glycol)*}

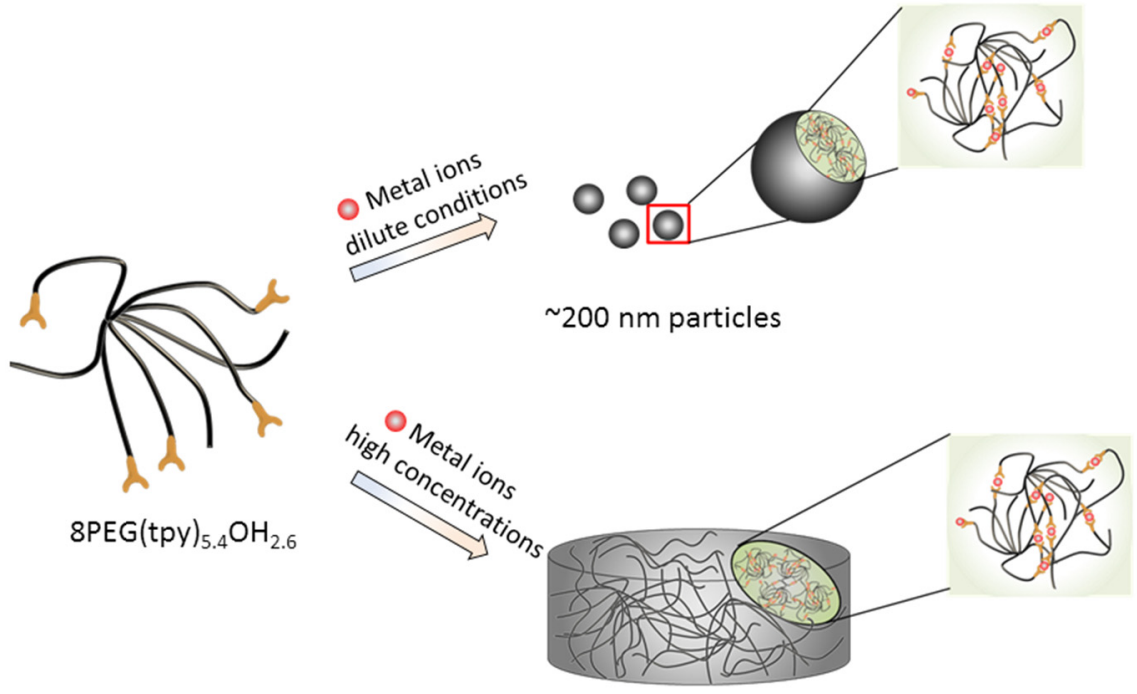

Hydrogels

* This chapter has been published: Wang, R; Geven, M.; Dijkstra, P. J.; Martens, P.; Karperien, M., Soft Matter 2014, 10 (37), 7328-7336. 


\section{Abstract}

Metallo supramolecular assemblies of an 8 arm poly(ethylene glycol) partially substituted with terpyridyl end-groups and the transition metal ions $\mathrm{Ni}^{2+}, \mathrm{Fe}^{2+}, \mathrm{Co}^{2+}$ and $\mathrm{Zn}^{2+}$ were studied for their nano-particle formation at dilute conditions and gelation at higher concentrations. The large differences in dissociation rate constants of the metal ligand complexes largely determines the assembly behavior. Thermodynamically stable complexes are generated with $\mathrm{Ni}^{2+}, \mathrm{Fe}^{2+}$ chlorides, which lead to distinct particle sizes of $\sim 200 \mathrm{~nm}$ in dilute conditions. The $\mathrm{Co}^{2+}$ and $\mathrm{Zn}^{2+}$ chlorides provide multiple size distributions revealing that mono and bis-complexes are present at equilibrium. Upon complexation, terpyridyl groups move to the outer sphere giving aggregates with a charged surface. At polymer concentrations above 5 wt\%, crosslinking upon addition of transition metal ions provides hydrogels. Elastic hydrogels were obtained with $\mathrm{Ni}^{2+}, \mathrm{Fe}^{2+}$ and $\mathrm{Co}^{2+}$ having storage moduli in excess of 20 $\mathrm{kPa}$, whereas $\mathrm{Zn}^{2+}$ gels are relatively viscous. Only $\mathrm{Zn}^{2+}$ gels show a thermoreversible sol to gel transition at a temperature of $25^{\circ} \mathrm{C}$ independent of polymer concentration. 


\subsection{Introduction}

Polymer gels are networks of polymer molecules that are either covalently or physically crosslinked and expanded throughout their volume by a fluid [1]. Physically crosslinked polymer gels are based on supramolecular chemistry, classically defined as the chemistry of complex formation through non-covalent interactions [2]. These interactions include dipole-dipole interactions, ionic bonding, hydrogen bonding, $\pi-\pi$ stacking or combinations of these, such as ion-dipole interactions $[2,3]$. The properties of physically cross-linked gels may vary widely, especially when the interactions are governed by dynamic equilibrium processes. As an example, cation-anion interactions can yield bond strengths up to $350 \mathrm{~kJ} / \mathrm{mol}$. This type of interaction has been used in several supramolecular polymer gels like calcium alginate gels [4,5], gellan gum [6] and chitosan polyelectrolyte complexes [7]. Although cation-anion interactions can yield mechanically strong gels, the cross-links in these gels can be dynamic due to the relatively high kinetic lability of the interaction [8]. This latter can result in interesting features of the gels, such as gelling transitions effected by temperature, $\mathrm{pH}$ or ultrasound and the ability to self-heal [9-11].

Gels formed by supramolecular cross-linking using metal ions as guests and ligands as hosts will also strongly depend on the predominant equilibrium state. A gel is only formed when the host-guest complex is favored at equilibrium. Bond strengths in metal ligand complexes can be as high as those of covalent bonds [12]. In certain cases highly stable complexes are formed when multiple Brønsted bases in a molecule donate electrons to a single Lewis acid which is known as the chelate effect $[13,14]$. Importantly, although these interactions can have a high thermodynamic stability, the metal coordination bond can nonetheless possess a relatively high kinetic lability. This combination of relatively high thermodynamic bond stability and kinetic lability makes metal coordination bonds an interesting choice for use as cross-links in polymeric gels. Such systems are currently attracting increasing interest and are termed metallosupramolecular polymer gels (MSPGs) [15].

Terpyridines are well known to effectively bind to transition metal ions, although reports of binding to alkali, alkaline earth and rare earth metal ions exist as well [16-19]. Binding with third row transition metal ions ideally proceeds in a two-step process [20]. 
The equilibria involve the stepwise coordination of two terpyridine moieties to a metal ion with different stability constants for the metal-mono(terpyridine) complex $\left(\mathrm{K}_{1}\right)$ and metal-bis(terpyridine) complex $\left(\mathrm{K}_{2}\right)$, respectively. The overall equilibrium is represented by the overall stability constant $\beta$, the product of $K_{1}$ and $K_{2}$. The stability constants are equilibrium constants and are defined by their association and dissociation rate constants [21]. The stability of transition metal bis(terpyridine) complexes is affected by several factors, such as the metal ion, temperature, $\mathrm{pH}$ and solvent. The metal ion is of paramount importance in this respect as it is known that the overall stability constants $(\beta)$ of transition metal ion-bis(terpyridine) complexes in water follows the series $\mathrm{Ni}^{2+}>\mathrm{Fe}^{2+}>\mathrm{Co}^{2+}>\mathrm{Zn}^{2+}[22,23]$.

Several extended overviews on terpyridine chemistry and their complexes with a variety of transition metal ions have been published in the past decade [24,25]. However, research on MSPGs has mainly focused on complex formation in organic solvents, i.e. on the formation of organogels and only minor research has been directed to hydrogels. Reports on MSPGs formed in water cover either terpyridyl end-group modified thermosensitive block copolymers, a terpyridyl grafted amphiphilic block copolymer and end-group modified 3 or 4 armed poly(ethylene glycol) [26]. Chiper et al. end modified commercially available pluronics with terpyridyl groups and determined their ability to form nano-particles in dilute solution upon complexation with $\mathrm{NiCl}_{2}$ [27]. The aqueous aggregation of these modified pluronics into micelles in the absence of a metal ion took place via hydrophobic interactions of the hydrophobic blocks and terpyridyl groups. Addition of $\mathrm{NiCl}_{2}$ led to the rearrangement and disruption of the micellar aggregates due to the hydrophilicity of the metal ion-terpyridine complexes generated. Furthermore, intramicellar bis(terpyridine) complexes were formed due to the relatively high effective concentration of these groups at the micelle coronas. At high concentrations of $20 \mathrm{wt} \%$, the pluronic F127 end modified with terpyridyl groups in the presence of half an equivalent $\mathrm{Ni}^{2+}$ ions showed thermoreversible gelation behaviour with a transition temperature of $34{ }^{\circ} \mathrm{C}$. As suggested, gelation depends on the packing of micelles and not on intermicellar $\mathrm{Ni}^{2+}$-terpyridine complexes formed.

Jochum et al. showed that a terpyridine modified block-copolymer of hydrophilic poly(triethyleneglycol methylether methacrylate) (PTEGMA) grafted with a low percentage of terpyridyl groups and polystyrene readily formed gels upon the addition 
of $\mathrm{NiCl}_{2}$ [28]. Compared to gels based on only terpyridine modified PTEGMA, the selfassembly of the polystyrene blocks led to additional cross-links resulting in an increased modulus of the gels.

Only a few papers on terpyridyl modified PEG's that can be crosslinked into MSPGs in an aqueous environment have appeared in literature. Schmatloch and Schubert reported on a low molecular weight three-arm PEG terminated with terpyridyl groups [26]. When combined with a linear terpyridyl end modified PEO, the addition of $\mathrm{Fe}^{2+}$ ions it was shown that the branched structure acted as a chain stopper instead of crosslinker. Kimura prepared 4-armed PEG, terminated with terpyridyl groups and studied the complexation behavior of this polymer with $\mathrm{Fe}^{2+}$ in methanol. The dried polymer formed a stable gel in water at a concentration of $20 \mathrm{wt} \%$. They also showed that varying the $\mathrm{pH}$ from 1 to 13 or heating to $90{ }^{\circ} \mathrm{C}$ did not give visible changes [29]. A three armed terpyridyl terminated PEG was recently studied for gelation with cobalt ions. Hydrogels were only formed from a water-soluble polymer having a Mn of 8000, but not with lower molecular weight polymers [30]. Recently, Yoshida and coworkers showed that terpyridyl end-modified 4 and 8 armed PEG form hydrogels upon complexation with $\mathrm{Ru}^{2+}$ ions. They used the complexes as a catalyst in the BelousovZhabotinsky reaction. The mechanical properties of the gels are almost independent on the number of arms, but gelation kinetics is higher for the 8-armed polymer [31]. Although the above presented examples show that terpyridyl-modified polymers may be used for the design of hydrogels, only minor attention has been given to the assembly process and stability of these systems in an aqueous environment using different transition metal ions. In a recent paper by Rossow and Seiffert supramolecular hydrogels based on 4-arm PEG end-modified with terpyridyl end-groups and $\mathrm{Mn}^{2+}, \mathrm{Zn}^{2+}$ and $\mathrm{Co}^{2+}$ ions were described [32]. They showed that network inhomogeneity is higher in $\mathrm{Co}^{2+}$ complexed gels compared to $\mathrm{Zn}^{2+}$ complexed gels. However, this nanometerscale inhomogeneity seems to enforce rather than weaken the gel mechanical properties. In this paper, we describe supramolecular gels formed by the self-assembly of an 8 arm PEG end modified with terpyridyl groups via metal-ligand interaction using $\mathrm{Ni}^{2+}, \mathrm{Fe}^{2+}$, $\mathrm{Co}^{2+}$ and $\mathrm{Zn}^{2+}$ ions. The aggregation at dilute aqueous conditions and gelation properties at concentrations above $5 \mathrm{wt} \%$ were determined. Importantly, we show that the kinetic 
stability of the metal-ligand crosslinks in the gels determines the temperature dependent gelation properties of the hydrogels.

\subsection{Experimental}

\subsubsection{Materials}

8 arm poly(ethylene glycol) (8PEG, hexaglycerol core, $\mathrm{Mw}=20,000)$ was acquired from Jenkem Technology (Allen, Texas, USA) and purified before use by dissolution in dichloromethane and precipitation in cold diethyl ether. The polymer was freeze dried overnight before use. Dimethyl sulfoxide (anhydrous, $\geq 99.9 \%$ ), 4'-chloro-2,2':6',2'”terpyridine $(99 \%)$, potassium hydroxide (BioXtra, $\geq 85 \%$ ), sodium sulfate (anhydrous, ACS reagent, $\geq 99 \%$ ), cobalt(II) chloride (anhydrous, 99.999\%), zinc chloride (anhydrous, $\geq 99.999 \%$ ), nickel(II) chloride hexahydrate (99.999\%) and iron(II) chloride (anhydrous, 99.99\%) were purchased from Sigma-Aldrich (Zwijndrecht, the Netherlands) and were used as received. Dichloromethane (AR, stabilized by Amylene, $\geq 99.9 \%$ ) and diethyl ether (AR, stabilized by BHT, $\geq 99.5 \%$ ) were from Biosolve and chloroform (for analysis, $\geq 99.8 \%$ ) was from Emsure and were used as received. Water used in this study was demineralized water purified by a Millipore Synergy system and bubbled with nitrogen gas for at least 30 minutes before use. Dialysis membranes (Spectra/Por molecular porous membrane tubing 4, MWCO: 12-14000) were acquired from Spectrum Laboratories, Inc. and were soaked in water for 30 minutes before use. Deuterated chloroform ( 99.8 atom\% D, $0.5 \mathrm{wt} \%$ silver foil as stabilizer) was from Aldrich. Durapore PVDF membrane, $0.22 \mu \mathrm{m}$ pore size filters were from Millex GV.

\subsubsection{Synthesis}

The 8 arm poly(ethylene glycol) end-group modified with terpyridyl groups was synthesized by the following procedure. Ground potassium hydroxide $(\mathrm{KOH}, 0.68 \mathrm{~g}$, $12.1 \mathrm{mmol})$ and 8PEG $(5.90 \mathrm{~g}, 0.30 \mathrm{mmol})$ were dissolved in $240 \mathrm{~mL}$ of dimethyl sulfoxide (DMSO) at $60{ }^{\circ} \mathrm{C}$ in a $\mathrm{N}_{2}$ atmosphere. After 90 min 4'-chloro-2,2':6',2'- 
terpyridine $(0.5 \mathrm{~g}, 1.87 \mathrm{mmol})$ was added and the reaction mixture was stirred for another 90 min at $60{ }^{\circ} \mathrm{C}$, during which the color of the solution changed from dark red to orange. The reaction mixture was cooled and the products precipitated in diethyl ether. After decantation, the sticky, slightly orange product was mixed with $125 \mathrm{~mL}$ of brine. The resulting mixture was extracted 4 times with $250 \mathrm{~mL}$ of chloroform and the combined organic layers were dried over sodium sulfate. After filtration, the chloroform was evaporated under reduced pressure and the remaining liquid was dissolved in dichloromethane and precipitated in cold diethyl ether. The product was dried in vacuum at room temperature (yield: $4.8 \mathrm{~g} ; 77 \%$ ). Additional purification of the product was performed by dissolving the polymer in water and subsequent dialysis against water for 24 hours. The $8 \mathrm{PEG}(\text { tpy })_{5.4} \mathrm{OH}_{2.6}$ was recovered by lyophilization. ${ }^{1} \mathrm{H}$ NMR (400 $\mathrm{MHz}, \mathrm{CDCl}_{3}$ ): $\delta=3.63$ (PEG protons), 3.92 (t, $2 \mathrm{H}, \mathrm{OCH}_{2} \mathrm{CH}_{2} \mathrm{O}$-terpyridyl), $4.46(\mathrm{t}, 2 \mathrm{H}$, $\mathrm{OCH}_{2} \mathrm{CH}_{2} \mathrm{O}$-terpyridyl), 7.32 (m, 2H, terpyridyl), 7.84 (dd, $2 \mathrm{H}$, terpyridyl), $8.03(\mathrm{~s}, 2 \mathrm{H}$, terpyridyl), $8.60(\mathrm{~d}, 2 \mathrm{H}$, terpyridyl), 8.69 ( $\mathrm{d}, 2 \mathrm{H}$, terpyridyl).

\subsection{Methods}

\subsubsection{UV-Vis titration}

Stock solutions of $8 \mathrm{PEG}(\text { tpy })_{5.4} \mathrm{OH}_{2.6}\left(0.2-0.5 \mathrm{mg} \mathrm{mL}^{-1}\right)$ were prepared in water. Solutions of the metal chloride salts in water were prepared at suitable concentrations that minimize dilution upon titration. In all experiments, after adding a selected amount of metal chloride solution the resulting solution was stirred for at least 5 min to allow equilibration. UV-Vis spectra were recorded using a Cary 300 UV-Vis spectrophotometer (Agilent technologies) at wavelengths between 200 and $800 \mathrm{~nm}$ at room temperature. The data assembled were corrected for dilution upon titration. The polymer concentration $\left(\mathrm{mg} \mathrm{mL}^{-1}\right)$, absorption coefficient $\left(\varepsilon\right.$ in $\left.\mathrm{L} \mathrm{mol}^{-1} \mathrm{~cm}^{-1}\right)$ and $\lambda \mathrm{c}(\mathrm{nm})$ for the different transition metal ion $8 \mathrm{PEG}(\text { tpy })_{5.4} \mathrm{OH}_{2.6}$ complexes were for: $\mathrm{NiCl}_{2}(0.30$, $17700,324), \mathrm{FeCl}_{2}(0.50,13400,556), \mathrm{CoCl}_{2}(0.25,20600,307)$, and $\mathrm{ZnCl}_{2}(0.20$, 27800, 322). 


\subsubsection{Dissociation rate constants}

Dissociation rate constants of $\mathrm{Fe}^{2+}$ and $\mathrm{Co}^{2+}-8 \mathrm{PEG}(\text { tpy })_{5.4} \mathrm{OH}_{2.6}$ complexes were determined according to a method described by Holyer et al. [22,33]. Stock solutions of $\mathrm{Fe}^{2+}$ and $\mathrm{Co}^{2+}-8 \mathrm{PEG}\left(\right.$ tpy) ${ }_{5.4} \mathrm{OH}_{2.6}$ complexes (metal ion:terpyridyl-groups is $1: 2 \mathrm{~mol} / \mathrm{mol}$ ) in water were prepared at a concentration of $0.78 \mathrm{mg} / \mathrm{mL}$. Subsequently, the resulting solution was added to a fused quartz cuvette and a 100 times molar excess of $\mathrm{NiCl}_{2} \cdot 6 \mathrm{H}_{2} \mathrm{O}$ or $\mathrm{FeCl}_{2}$ in $50 \mu \mathrm{l}$ of water was added. The change in the absorption at 556 $\mathrm{nm}$ (exchange of $\mathrm{Fe}^{2+}$ by $\mathrm{Ni}^{2+}$ ) or increase in the absorption at $556 \mathrm{~nm}$ (exchange of $\mathrm{Co}^{2+}$ by $\mathrm{Fe}^{2+}$ ) were recorded over time at room temperature.

\subsubsection{Dynamic light scattering (DLS)}

Aqueous solutions of $8 \mathrm{PEG}(\mathrm{tpy})_{5.4} \mathrm{OH}_{2.6}$ were prepared at a concentration of $3 \mathrm{mg} / \mathrm{ml}$. A titration experiment was performed by adding set amounts of metal chloride solution under stirring at room temperature to give $\mathrm{M}^{2+}$ :terpyridyl-groups molar ratios of $0,1: 8$, $3: 8,1: 2,3: 4$ and 1:1. After addition of an aliquot of the metal ion solution the resulting solution was equilibrated overnight. Size distributions were recorded on a Malvern Instruments Zetasizer Nano ZS at $20{ }^{\circ} \mathrm{C}$ and a $173^{\circ}$ backscatter angle. Solutions containing a $\mathrm{M}^{2+}$ :terpyridyl-groups molar ratio of 1:2 were used to measure the zeta potential. The zeta potentials were recorded (Malvern Instruments Zetasizer Nano ZS) at $20{ }^{\circ} \mathrm{C}$, using a $633 \mathrm{~nm} \mathrm{He}-\mathrm{Ne}$ laser and backscattering detection. Polystyrene capillary folded cells were used.

\subsubsection{Rheometry}

Oscillatory rheometry experiments were performed on an Anton Paar, Physica MCR201 system, using a PP25 measuring plate ( $25 \mathrm{~mm}$ diameter). Approximately $300 \mu \mathrm{l}$ of a gel with a concentration of either 5,10 or $20 \mathrm{wt} \%$ were prepared by dissolution of $8 \mathrm{PEG}(\text { tpy })_{5.4} \mathrm{OH}_{2.6}$ in water and addition of a calculated amount of metal chloride solution $\left(\mathrm{M}^{2+}\right.$ :terpyridyl-groups molar ratio of 1:2). The gels were left overnight at room 
temperature. A gel was placed at the center of the measuring plate at $5{ }^{\circ} \mathrm{C}$ and the measuring gap was set to $0.3 \mathrm{~mm}$, and excess gel was removed. The system was equipped with an oil based solvent trap and the storage ( $\left.\mathrm{G}^{\prime}\right)$ and loss modulus (G') were recorded under oscillatory shear. Measurements were performed at a frequency $(\omega)$ of 1 $\mathrm{Hz}$ and strain $(\gamma)$ of $1 \%$. Subsequently, heating and cooling cycles were performed between 5 and $60{ }^{\circ} \mathrm{C}$ at a rate of $1{ }^{\circ} \mathrm{C} / \mathrm{min}$. After the temperature cycles, samples were cooled to $20^{\circ} \mathrm{C}$ and a frequency and amplitude sweeps were performed between $\omega=$ $0.1-100 \mathrm{~Hz}(\gamma=1 \%)$ and $\gamma=0.01-100 \%(\omega=1 \mathrm{~Hz})$ to confirm that all samples were within the linear viscoelastic regime.

\subsection{5 ${ }^{1} \mathrm{H}-\mathrm{NMR}$}

Proton nuclear magnetic resonance $\left({ }^{1} \mathrm{H}-\mathrm{NMR}\right)$ spectra were recorded on a Bruker Ascend 400/Avance III $400 \mathrm{MHz}$ NMR spectrometer system using deuterated chloroform as a solvent. Solutions were prepared at a concentration of approximately 10 $\mathrm{mg} / \mathrm{ml}$ and measurements were performed at ambient temperature.

\subsection{Results and discussion}

\subsubsection{Synthesis}

The hydroxyl groups of the star shaped 8PEG were partly converted into terpyridylgroups by a nucleophilic aromatic substitution reaction of potassium hydroxide generated alkoxide groups of the 8PEG to 4'-chloro-2,2':6',2'-terpyridine as depicted in Figure 6.1. The reaction was performed for $1 \mathrm{~h}$ and within this time period a color change can be observed from dark red to orange. The final ratio of hydroxyl to terpyridyl-groups was controlled by the molar feed ratio of the reactants, although full conversion of the hydroxyl groups could not be obtained when an excess of the chloroterpyridine was used. The reaction was performed in such a way that approximately 5 of the 8 hydroxyl groups were substituted with terpyridyl-groups. The ${ }^{1} \mathrm{H}-\mathrm{NMR}$ spectrum confirmed the structure of the product (Figure 6.2) and the number of substituted 
hydroxyl groups was calculated from the ratio of the terpyridine protons (a-e) and the $\mathrm{CH}_{2}$-groups of the terminal PEG oxy ethyl unit ( $\mathrm{f}$ and $\mathrm{g}$ ). Of the 8 hydroxyl groups initially present on average 5.4 hydroxyl groups per polymer molecule were substituted by terpyridyl-groups affording $8 \mathrm{PEG}\left(\operatorname{tpy}_{5.4}\right) \mathrm{OH}_{2.6}$.

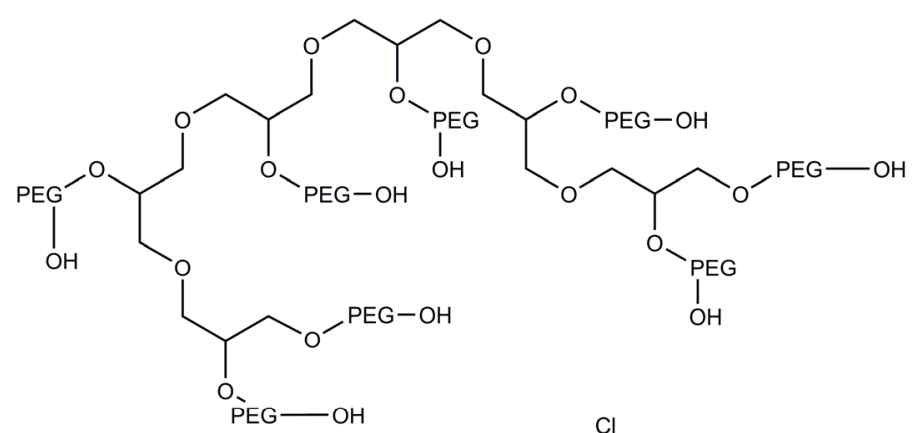<smiles>O=S(=O)(O)O[Na]</smiles>

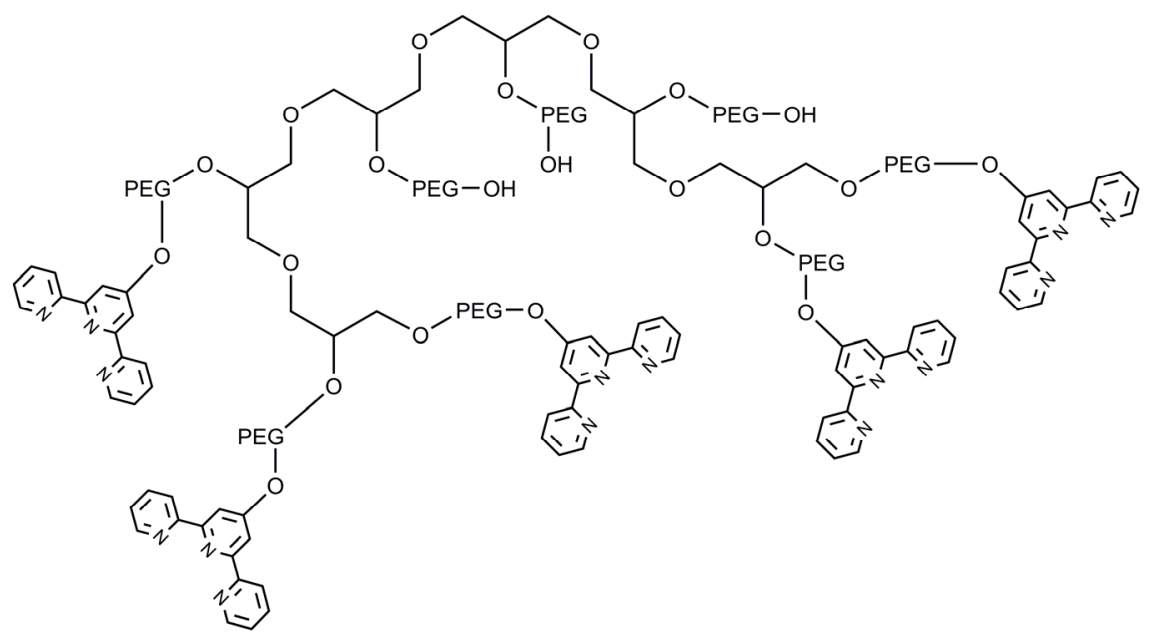

Figure 6.1 Synthesis of an 8 PEG partially substituted with terpyridyl-groups. 

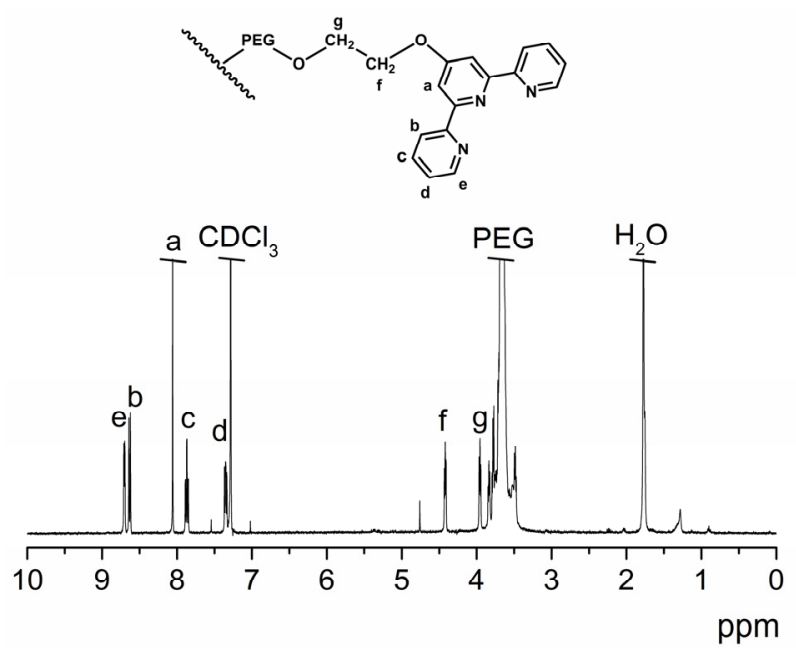

Figure 6.2 ${ }^{1} \mathrm{H}-\mathrm{NMR}$ spectrum of $8 \mathrm{PEG}\left(\mathrm{tpy}_{5.4}\right) \mathrm{OH}_{2.6}\left(\mathrm{CDCl}_{3}\right)$.

\subsubsection{Characterization of complexion with transition metal ions}

To determine complexation ratios of the $8 \mathrm{PEG}\left(\right.$ tpy $\left._{5.4}\right) \mathrm{OH}_{2.6}$ with $\mathrm{Ni}(\mathrm{II}), \mathrm{Fe}(\mathrm{II}), \mathrm{Co}(\mathrm{II})$ and $\mathrm{Zn}(\mathrm{II})$ ions in water, UV-Vis titration experiments were carried out. Moreover, given the known high kinetic stability of the $\mathrm{Ni}^{2+}$ bis(terpyridine) complexes, the titration with a $\mathrm{NiCl}_{2}$ solution was used to determine the average number of terpyridylgroups per polymer molecule. Upon titration, the evolution of the characteristic adsorption band of the $\mathrm{Ni}^{2+}$-complex at $324 \mathrm{~nm}$ (Figure 6.3A) was used to determine the molar complex ratio. By definition, the $\mathrm{Ni}^{2+}$-complex absorption will reach a

maximum when all terpyridyl-groups are complexed. As depicted in Figure 6.4A, at a ratio of $\mathrm{Ni}^{2+}$ to terpyridyl-groups of $1: 2$, the absorption indeed remains constant upon further addition of $\mathrm{Ni}^{2+}$ ions,. The number of terpyridyl-groups coupled to the 8PEG can then be calculated from this maximum (i.e., from the intercept of the straight lines). The average number of coupled terpyridyl-groups was calculated as 5.4, which is similar to that which was determined by ${ }^{1} \mathrm{H}-\mathrm{NMR}$. 

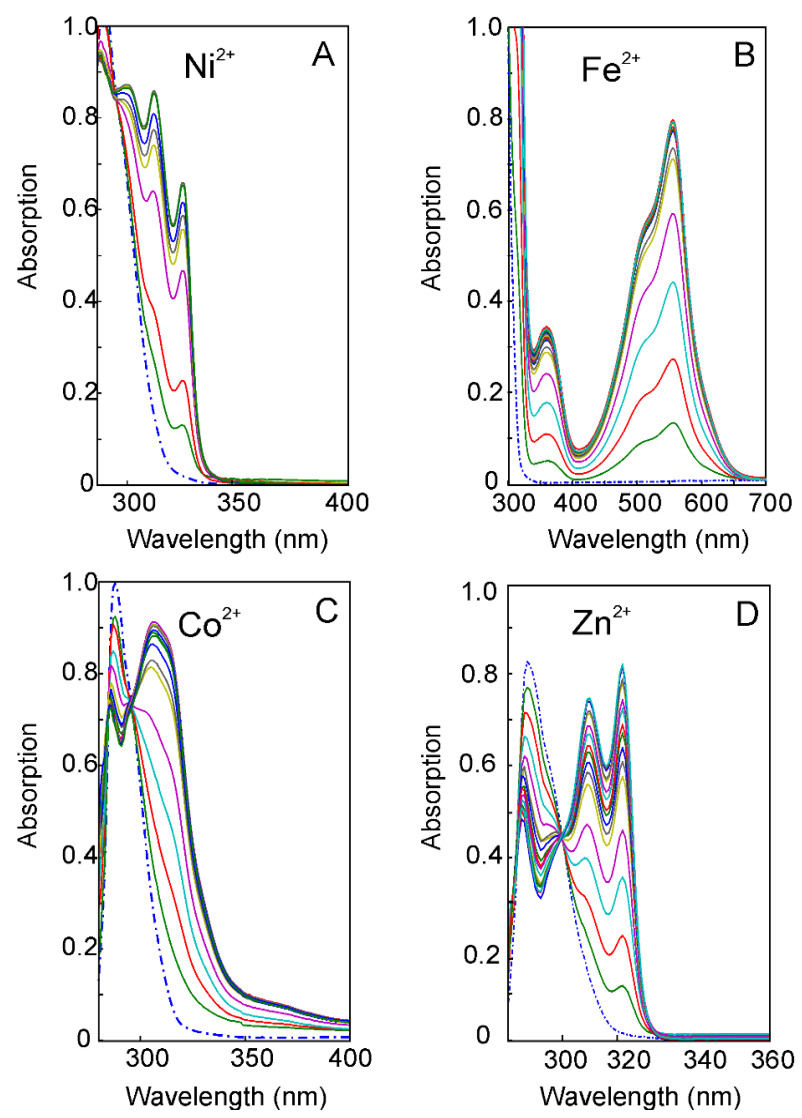

Figure 6.3 UV-Vis absorption spectra recorded for the titration of a $8 \mathrm{PEG}\left(\operatorname{tpy}_{5.4}\right) \mathrm{OH}_{2.6}$ solution with a $\mathrm{NiCl}_{2}(\mathrm{~A}), \mathrm{FeCl}_{2}(\mathrm{~B}), \mathrm{CoCl}_{2}$ (C) and $\mathrm{ZnCl}_{2}$ solution (D). Dotted lines are of the uncomplexed polymer.

Titrations with the other transition metal chlorides, $\mathrm{Fe}^{2+}, \mathrm{Co}^{2+}$, and $\mathrm{Zn}^{2+}$ were performed similarly. From the characteristic $\mathrm{Fe}^{2+}, \mathrm{Co}^{2+}$ and $\mathrm{Zn}^{2+}$ bis-terpyridyl metal ligand charge transfer absorption bands at $556 \mathrm{~nm}, 307 \mathrm{~nm}$ and $322 \mathrm{~nm}$, respectively (Figure 6.3 B, C \& D), the complex ratios were determined (Figure 6.4 B, C \& D). Titration with $\mathrm{Fe}^{2+}$ also demonstrated a maximum complexation ratio of 1:2, whereas the metal ion:terpyridyl-groups ratios were somewhat higher for the $\mathrm{Co}^{2+}$ and $\mathrm{Zn}^{2+}$ complexes. This is a clear indication that $\mathrm{Fe}^{2+}$ forms stable bis-terpyridyl complexes with 8PEG(tpy $\left.{ }_{5.4}\right) \mathrm{OH}_{2.6}$ in water, whereas in the case of $\mathrm{Co}^{2+}$ or $\mathrm{Zn}^{2+}$ mono-complexes may also be present [34]. Moreover, the decrease of the absorption at higher 
$\mathrm{Co}^{2+}$ :terpyridyl-groups ratios is indicative of a lower stability of cobalt complexes compared to iron and nickel complexes. Similarly, adding an excess of $\mathrm{Zn}^{2+}$ ions results in a decrease of the absorption at $322 \mathrm{~nm}$, indicative of the formation of monocomplexes [20].
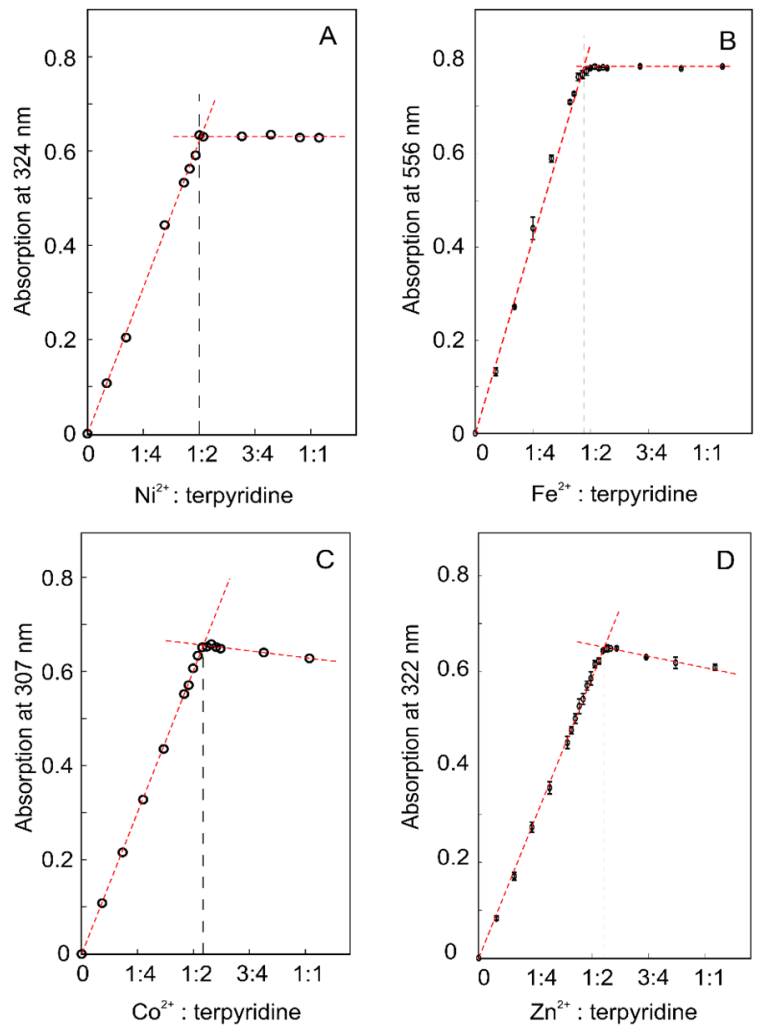

Figure 6.4 UV-Vis absorption spectra recorded for the titration of a $8 \mathrm{PEG}\left(\operatorname{tpy}_{5.4}\right) \mathrm{OH}_{2.6}$ solution with a $\mathrm{NiCl}_{2}$ (A), $\mathrm{FeCl}_{2}$ (B), $\mathrm{CoCl}_{2}$ (C) and $\mathrm{ZnCl}_{2}$ solution (D). Absorption values were determined at $324 \mathrm{~nm}\left(\mathrm{Ni}^{2+}\right)$, at $556 \mathrm{~nm}\left(\mathrm{Fe}^{2+}\right)$, at $307 \mathrm{~nm}\left(\mathrm{Co}^{2+}\right)$ and at 322 $\mathrm{nm}\left(\mathrm{Zn}^{2+}\right)$.

\subsubsection{Determination of dissociation rate constants}

In the titration experiments, complex formation appears quickly and may even be diffusion controlled. Rate constants for complex formation of transition metal ions and 
terpyridyl-groups have been determined in the past and are generally $10^{4} \mathrm{M}^{-1} \cdot \mathrm{s}^{-1}$ [22]. The differences observed in the titration experiments with an excess of metal ions are therefore indicative of differences in the dissociation rate constants. Because the dissociation rate constants were expected to have an influence on the aggregation at dilute conditions and crosslinking into hydrogels at higher concentrations (vide infra), the dissociation rate constants of the transition metal complexes of the polymers in water were first determined.

Metal exchange experiments in dilute solutions were performed according to a procedure described by Henderson and Hayward [33]. $\mathrm{Fe}^{2+}$-bis(terpyridyl) complexes were dissociated with a 100 -fold excess $\mathrm{Ni}^{2+}$ and of $\mathrm{Co}^{2+}$-bis(terpyridyl) complexes were dissociated with a 100 -fold excess $\mathrm{Fe}^{2+}$. In both cases, the absorption band of the $\mathrm{Fe}^{2+}$-bis(terpyridyl) complexes was followed by UV-Vis spectroscopy. Henderson and Hayward have shown that these exchanges enable the determination of dissociation rate constants of the $\mathrm{Fe}^{2+}$ and $\mathrm{Co}^{2+}$ bis(terpyridyl) complexes.

$O=\mathrm{M}_{1}$, the weaker complex forming metal ion

1)

$\hookrightarrow \odot) \frac{k_{-2,1}}{k_{2,1}}$<smiles>C=CO[Te]C1=CC=CC1</smiles>

$\mathrm{O}=\mathrm{M}_{2}$, the stronger complex forming metal ion

2) $\int+0 \frac{k_{1,2}}{k_{-1,2}}$
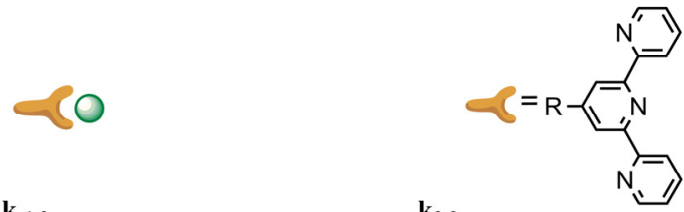

3)
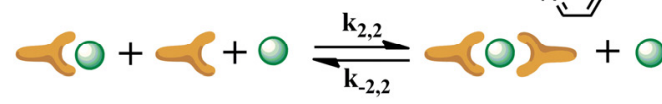

Figure 6.5 Equilibria involved in the metal ion exchange of bis(terpyridyl) complexes.

Rate constants are indicated by $\mathrm{k}_{\mathrm{m}, \mathrm{n}}$, in which $\mathrm{m}$ refers to a mono (1) or bis-complex (2) and $\mathrm{n}$ refers to the metal ion as depicted in the figure.

The dissociation and association of the metal ion bis(terpyridyl) complexes in the presence of an excess of stronger complexing metal ions can be described by a series of equilibrium reactions as depicted in Figure 6.5. During metal ion exchange the initial bis-complex dissociates (equilibrium 1) and the resulting free terpyridyl-groups rapidly forms a mono-complex with the stronger complexing metal ion (equilibrium 2). The 
mono-complexes subsequently disproportionate and bis-complexes are finally formed (equilibrium 3).

Equations describing the changes in $\mathrm{Fe}^{2+}$-bis(terpyridyl) concentration in time were derived for either the exchange of $\mathrm{Fe}^{2+}$-bis(terpyridyl) to $\mathrm{Ni}^{2+}$-bis(terpyridyl) complexes (Eq. 1) or $\mathrm{Co}^{2+}$-bis(terpyridyl) to $\mathrm{Fe}^{2+}$-bis(terpyridyl) complexes (Eq. 2). Details for deriving these equations are given in Appendix in the end of this chapter. In these equations, $\mathrm{k}$ 2 is the dissociation rate constant in $\mathrm{s}^{-1}$ and $\mathrm{t}$ is time in sec. Using Matlab ${ }^{\circledR}$ scripts, Eq. 1 and 2 were fitted to the collected data (Figure 6.6).

$$
\begin{aligned}
& {\left[\mathrm{Fe}^{2+}(\text { tpy })_{2}\right]_{t}=\left[\mathrm{Fe}^{2+}(\text { tpy })_{2}\right]_{0} e^{-k-2, F e t}} \\
& {\left[\mathrm{Fe}^{2+}(\text { tpy })_{2}\right]_{t}=1 / 2\left[\mathrm{Co}^{2+}(\text { tpy })_{2}\right]_{0}\left(1-e^{-k-2, \mathrm{Cot}}\right)}
\end{aligned}
$$

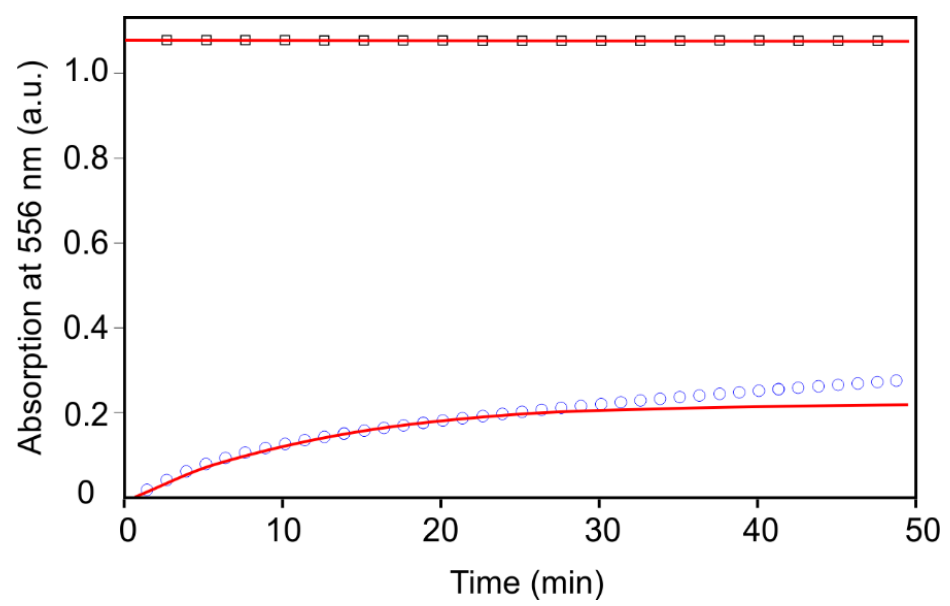

Figure 6.6 Absorption at $556 \mathrm{~nm}$ as a function of time of the $\mathrm{Fe}^{2+}$ to $\mathrm{Ni}^{2+}$ exchange (squares) and of the $\mathrm{Co}^{2+}$ to $\mathrm{Fe}^{2+}$ exchange (circles). Lines represent fits of the measured data.

The exchange of $\mathrm{Co}^{2+}$ by $\mathrm{Fe}^{2+}$ ions from the $\mathrm{Co}^{2+}$-bis(terpyridyl) complex proceeds relatively quickly and could be accurately fit over the first 20 minutes of the metal ion exchange. Within this time span, metal ion exchange is dominated by $\mathrm{Co}^{2+}-$ bis(terpyridyl) dissociation. Thereafter, the data deviate from the fit due to $\mathrm{Co}^{2+}$ - 
mono(terpyridyl) dissociation. The fit yields a dissociation rate constant $\mathrm{k}_{-2, \mathrm{Co}}$ of $1.4 \cdot 10^{-3}$ $\mathrm{s}^{-1}$, similar to the value presented in literature for the dissociation rate constant of a $\mathrm{Co}^{2+}$ complex of a linear terpyridyl end-group modified PEG $\left(1.5 \cdot 10^{-3} \mathrm{~s}^{-1}\right)$ [33]. The exchange of $\mathrm{Fe}^{2+}$ by $\mathrm{Ni}^{2+}$ ions from the $\mathrm{Fe}^{2+}$-bis(terpyridyl) complex proceeds rather slowly and the data could be fit according to Eq. 1 over at least 6 hours, yielding a dissociation rate constant of $8.1 \cdot 10^{-7} \mathrm{~s}^{-1}$. This dissociation rate constant is lower than that determined for a $\mathrm{Fe}^{2+}$ complex of terpyridyl end-group modified linear PEG $\left(5.0 \cdot 10^{-6} \mathrm{~s}^{-1}\right)$ but higher than that of an unsubstituted terpyridine $\left(1.6 \cdot 10^{-7}\right)[22,33]$. The differences observed in the dissociation rate constants could be a result of more effective shielding of the bis(terpyridyl) complexes by the 8 arm polymer. This may limit the accessibility of the complexes by metal ions in solution. The dissociation rate constants of bis(terpyridyl) complexes with $\mathrm{Ni}^{2+}$ and $\mathrm{Zn}^{2+}$ could not be determined. For the $\mathrm{Ni}^{2+}$ complexes this is due to their high stability. The $\mathrm{Zn}^{2+}$ complexes have too low a stability and mainly free ligands are present (equilibrium 1 in Figure 6.5 is to the right).

\subsubsection{Complexation behavior at dilute conditions}

In dilute aqueous solutions $(3 \mathrm{mg} / \mathrm{ml}), 8$ PEG-TEP $5.4 \mathrm{OH}_{2.6}$ forms nano-particles with an average diameter of $\sim 10 \mathrm{~nm}$. Please note that intensity plots do not provide ratios of particle size distributions, as larger particles scatter more than small particles. However, such plots give insight into changes in aggregation phenomena that can be caused by structural changes. The particle size of the 8 PEG-TEP ${ }_{5.4} \mathrm{OH}_{2.6}$ as measured with DLS appeared similar to that of the 8 armed $\mathrm{PEG}(\mathrm{OH})_{8}$ revealing that the polymer does not aggregate in water at dilute conditions. Titration of $8 \mathrm{PEG}\left(\operatorname{tpy}_{5.4}\right) \mathrm{OH}_{2.6}$ with the transition metal ions $\mathrm{Ni}^{2+}$ and $\mathrm{Fe}^{2+}$ resulted in a distinct shift of the average particle size (Figure 6.7 A \& B). Upon titration, a new distribution of particles with an average size of $\sim 200 \mathrm{~nm}$ appears, whereas the distribution of the small particles of $\sim 10 \mathrm{~nm}$ shifts to lower values with decreased intensities. A high resolution SEM picture of $\mathrm{Fe}^{2+}$ complexed particles is presented in Figure 6.8 and this is consistent with data from DLS measurements. 

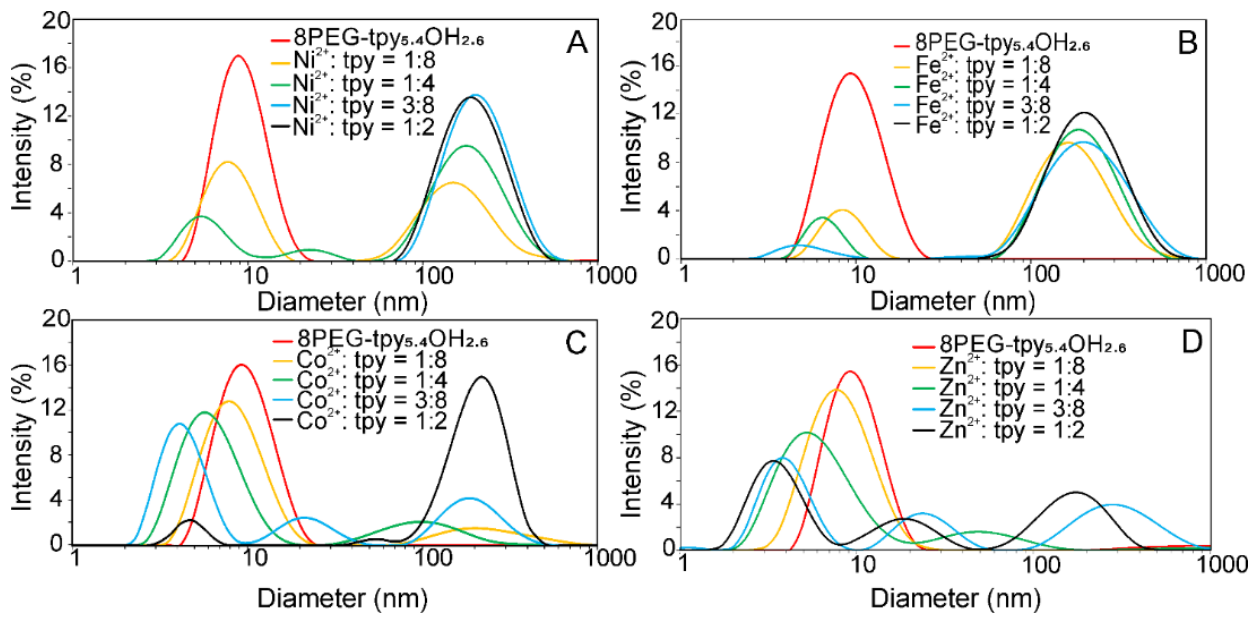

Figure 6.7 Nano-particle formation of $8 \mathrm{PEG}\left(\operatorname{tpy}_{5.4}\right) \mathrm{OH}_{2.6}$ with $\mathrm{Ni}^{2+}(\mathrm{A}), \mathrm{Fe}^{2+}(\mathrm{B}), \mathrm{Co}^{2+}$ (C) and $\mathrm{Zn}^{2+}$ (D) chlorides at different ratios probed by DLS.

The distinct change in size distribution indicates the formation of $\mathrm{Ni}^{2+}$-terpyridyl complexes disrupting the hydrophobic interactions between terpyridyl-groups and causing rearrangement to minimize the surface free energy. At a $\mathrm{Ni}^{2+}$ :terpyridyl-groups ratio of 1:2 groups the smaller particles are no longer present and addition of an excess metal ions does not result in changes of the particle size distribution. The decrease in the average diameter of the small particles $(\sim 10 \mathrm{~nm})$ upon addition of $\mathrm{Ni}^{2+}$ ions is similar as observed by Chiper et al. on terpyridyl end-group modified pluronics [27]. They explained this decreasing average particle size by rearrangement into intramicellar complexes, generating flower type micelles. Here, the distinct change to particles with an average diameter of $\sim 200 \mathrm{~nm}$ upon titration also reveals a fast rearrangement. Likely the $\mathrm{Ni}^{2+}$ - complexes formed rearrange to the outer surface of the particles. Both the charge distribution and shielding of charges by PEG may contribute to the stability of the particles (vide supra).

Contrary to the distinct complete change in size distribution upon titration with $\mathrm{Fe}^{2+}$ or $\mathrm{Ni}^{2+}$ chlorides, the titration with $\mathrm{Co}^{2+}$ or $\mathrm{Zn}^{2+}$ chlorides yields nano-particle suspensions with multiple size distributions (Figure 6.7 C \& D). Similarly as described above, upon the addition of $\mathrm{Co}^{2+}$ the average size of the small particles is shifted to lower values. However, the formation of larger particles is less pronounced. Intermediate sized particles (average diameter of $20 \sim 100 \mathrm{~nm}$ ) are observed during 
titration at $\mathrm{Co}^{2+}$ :terpyridyl-groups ratios of 1:4 and 3:8. Furthermore, small micellar type nano-particles with an average diameter of $5 \mathrm{~nm}$ are still present at a $\mathrm{Co}^{2+}$ :terpyridyl-groups ratio of $1: 2$. Even adding an excess of $\mathrm{Co}^{2+}$ relative to the amount of terpyridyl-groups results in a narrowing of the particle size distribution at $200 \mathrm{~nm}$ diameter while the small particle distribution at $5 \mathrm{~nm}$ still present (data not shown). The larger variation of the nano-particle size distribution is likely caused by the relatively low stability of the $\mathrm{Co}^{2+}$-terpyridyl complexes compared to the $\mathrm{Ni}^{2+}$-terpyridyl and $\mathrm{Fe}^{2+}$-terpyridyl complexes in water. Possibly, exchange of $\mathrm{Co}^{2+}$ ions between terpyridyl-groups occurs, allowing the formation of both mono-complexes and biscomplexes into intermediate sized particles. Upon addition of distinct volumes of a $\mathrm{Zn}^{2+}$ chloride solution, similar changes were observed. The multimodal size distributions observed indicate that both mono and bis-complexes are present at equilibrium. At higher metal ion to terpyridyl-groups ratios only small changes were observed in the size distribution of the $\mathrm{Zn}^{2+}$ and $\mathrm{Co}^{2+}$ complexes, the distribution at $200 \mathrm{~nm}$ becoming more narrow, whereas the distribution of the $\mathrm{Fe}^{2+}$ and $\mathrm{Ni}^{2+}$ complexes did not change.

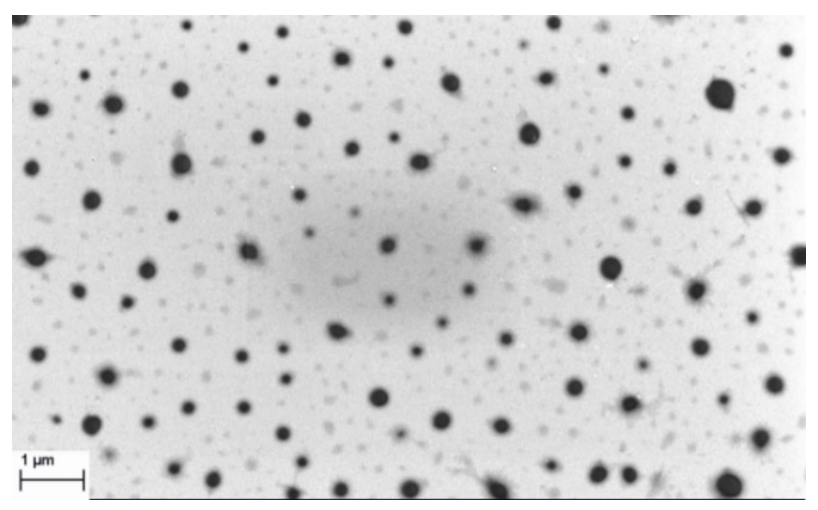

Figure 6.8 SEM image of $\mathrm{Fe}^{2+}$ complexed 8PEG(tpy $\left.{ }_{5.4}\right) \mathrm{OH}_{2.6}(1: 2)$ nanoparticles.

The changes in size distribution by complex formation of the terpyridyl-groups with transition metal ions indicate the reorganization of the initially present micelles. The zeta potentials were determined at a metal ion:terpyridyl-groups ratio of $1: 2$ and revealed significant differences between the particle or particle distributions formed with $\mathrm{Fe}^{2+}$ or $\mathrm{Ni}^{2+}$ and those formed with $\mathrm{Zn}^{2+}$ or $\mathrm{Co}^{2+}$. For the latter the determined zeta potentials gave an average positive surface charge of 5 and $8 \mathrm{mV}$, respectively. The $\mathrm{Ni}^{2+}$ 
and $\mathrm{Fe}^{2+}$-terpyridyl complexes form only larger particles at this ratio providing a larger positive charge of $17 \sim 18 \mathrm{mV}$. Although particle suspensions prepared by $\mathrm{Ni}^{2+}$ and $\mathrm{Fe}^{2+}$ terpyridine complexation are more stable than those comprising $\mathrm{Zn}^{2+}$ or $\mathrm{Co}^{2+}$ complexes, they will still tend to flocculate over time as their zeta potential is lower than $30 \mathrm{mV}$.

\subsubsection{Hydrogel mechanical properties}

Addition of transition metal ions to aqueous solutions of $8 \mathrm{PEG}\left(\operatorname{tpy}_{5.4}\right) \mathrm{OH}_{2.6}$ at concentrations higher than $3 \mathrm{wt} \%$ produced hydrogels instead of discrete particles. Gelation took place instantaneously. Previous research on three and four armed poly(ethylene glycol)s having terpyridyl end-groups indicated that stable gels could be obtained with either $\mathrm{Co}^{3+}$ or $\mathrm{Fe}^{2+}$ [26,30], but the mechanical properties of the gels were not studied. Considering the large differences in aggregation behavior, we studied the temperature dependent mechanical properties of the hydrogels by oscillatory rheology (see Table 6.1). The effect of temperature on the gels was assessed by measuring changes in the storage (G') and loss (G') moduli between 5 and $60{ }^{\circ} \mathrm{C}$ applying cyclic temperature measurements. Such cyclic measurements can show that the gels are in equilibrium and may show temperature dependent gelation. The mechanical properties presented in Table 6.1 are from the last cooling cycle.

The damping factor of the $\mathrm{Ni}^{2+}$ and $\mathrm{Fe}^{2+}$ gels varies from approximately $1 \cdot 10^{-3}$ to $5 \cdot 10^{3}$, which demonstrates that elastic hydrogels were obtained. The much higher damping factor of the $\mathrm{Co}^{2+}$ gels at higher temperatures and $\mathrm{Zn}^{2+}$ gels reveals a much more viscous behavior.

As depicted in Figure 6.9 the storage moduli of the $\mathrm{Ni}^{2+}$ and $\mathrm{Fe}^{2+}$ gels increase with an increase in temperature according the theory of rubber elasticity (Eq. 3). In this equation $\mathrm{G}^{\prime}$ is the storage modulus in $\mathrm{Pa}, \rho$ is the cross-link density in moles of elastically effective network chains per $\mathrm{m}^{3}, \mathrm{R}$ is the gas constant $\left(8.314 \mathrm{~J} \cdot \mathrm{K}^{-1} \cdot \mathrm{mol}^{-1}\right)$ and $\mathrm{T}$ is the temperature in $\mathrm{K}$.

$$
G^{\prime}=\rho R T
$$


Table 6.1 Storage and loss moduli of $\mathrm{Ni}^{2+}, \mathrm{Fe}^{2+}, \mathrm{Co}^{2+}$ and $\mathrm{Zn}^{2+} 8 \mathrm{PEG}\left(\operatorname{tpy}_{5.4}\right) \mathrm{OH}_{2.6}$ hydrogels.

\begin{tabular}{|c|c|c|c|c|c|}
\hline Metal ion & $\begin{array}{l}\text { Polymer } \\
(\mathrm{wt} \%)\end{array}$ & $\begin{array}{c}\text { Temperature } \\
\left({ }^{\circ} \mathrm{C}\right)\end{array}$ & $\begin{array}{c}\mathrm{G}^{\prime} \\
(\mathrm{Pa})\end{array}$ & $\begin{array}{l}\text { G” } \\
(\mathrm{Pa})\end{array}$ & $\begin{array}{l}\tan \delta \\
\times 10^{3}\end{array}$ \\
\hline \multirow{4}{*}{$\mathrm{Ni}^{2+}$} & \multirow{2}{*}{5} & 10 & 3100 & 4 & 1.3 \\
\hline & & 60 & 6100 & 8 & 1.3 \\
\hline & \multirow{2}{*}{10} & 10 & 9300 & 13 & 1.4 \\
\hline & & 60 & 8200 & 50 & 6.1 \\
\hline \multirow{6}{*}{$\mathrm{Fe}^{2+}$} & \multirow{2}{*}{5} & 10 & 3200 & 10 & 3.1 \\
\hline & & 60 & 5500 & 20 & 3.7 \\
\hline & \multirow{2}{*}{10} & 10 & 8200 & 18 & 2.2 \\
\hline & & 60 & 12500 & 35 & 2.8 \\
\hline & \multirow{2}{*}{20} & 10 & 21000 & 56 & 2.7 \\
\hline & & 60 & 28000 & 116 & 4.1 \\
\hline \multirow{6}{*}{$\mathrm{Co}^{2+}$} & \multirow{2}{*}{5} & 10 & 3600 & 17 & 4.7 \\
\hline & & 60 & 2700 & 230 & 85 \\
\hline & \multirow{2}{*}{10} & 10 & 5100 & 8 & 1.5 \\
\hline & & 60 & 6100 & 250 & 41 \\
\hline & \multirow{2}{*}{20} & 10 & 6900 & 10 & 1.1 \\
\hline & & 60 & 8900 & 340 & 38 \\
\hline \multirow{6}{*}{$\mathrm{Zn}^{2+}$} & \multirow{2}{*}{5} & 10 & 2000 & 620 & 310 \\
\hline & & 60 & 7 & 55 & 7900 \\
\hline & \multirow{2}{*}{10} & 10 & 13000 & 3100 & 240 \\
\hline & & 60 & 110 & 1200 & 11000 \\
\hline & \multirow{2}{*}{20} & 10 & 28000 & 6900 & 250 \\
\hline & & 60 & 8800 & 5000 & 570 \\
\hline
\end{tabular}



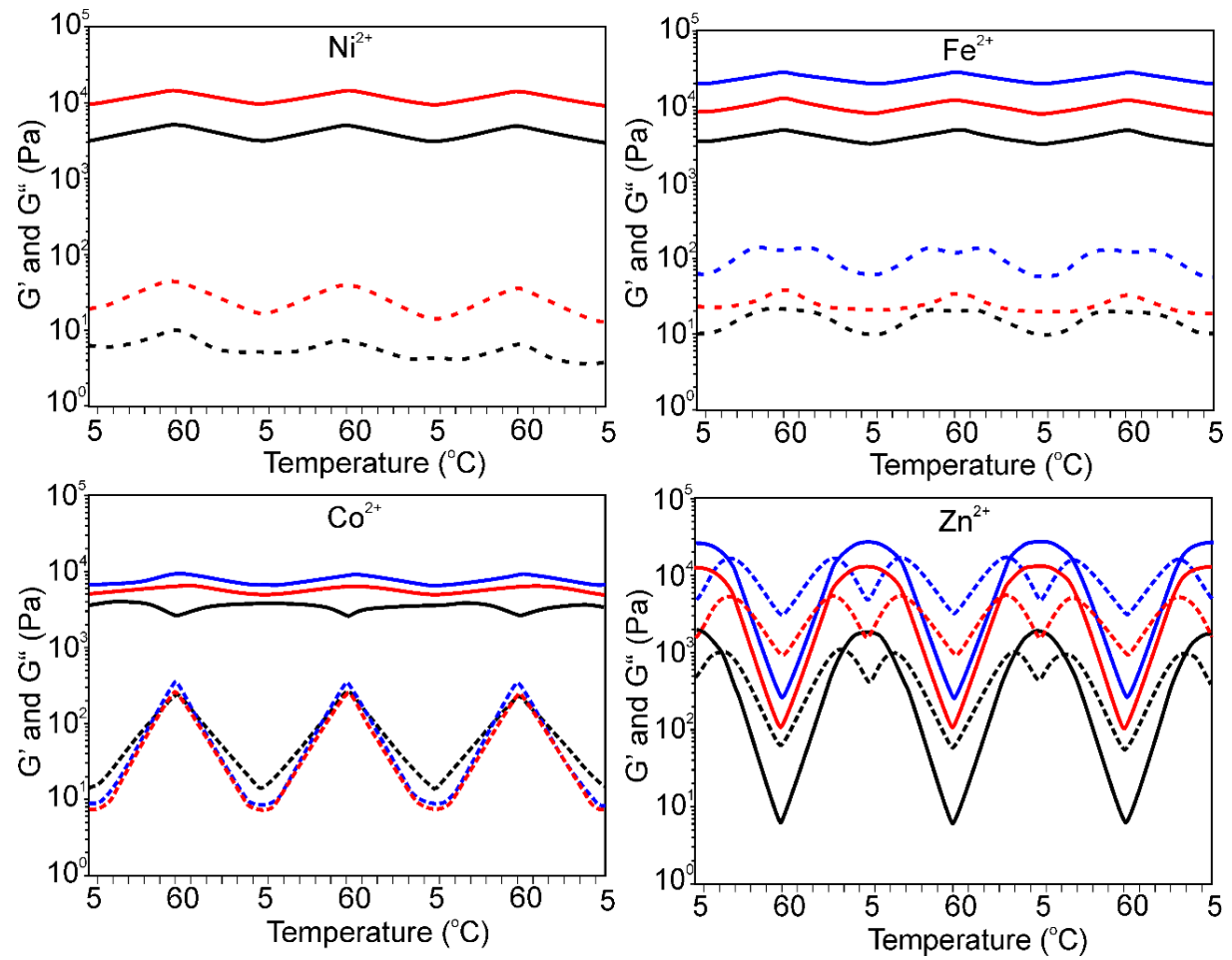

Figure 6.9 Temperature dependent storage ( $G^{\prime}$, solid line) and loss ( $G^{\prime \prime}$, dotted line) modulus of $\mathrm{Ni}^{2+}, \mathrm{Fe}^{2+}, \mathrm{Co}^{2+}$ or $\mathrm{Zn}^{2+} 8 \mathrm{PEG}\left(\right.$ tpy $\left.{ }_{5.4}\right) \mathrm{OH}_{2.6}\left(\mathrm{M}^{2+}\right.$ :terpyridyl-groups = 1:2) hydrogels upon heating to $60{ }^{\circ} \mathrm{C}$ and subsequent cooling to $5{ }^{\circ} \mathrm{C}$ (three cycles) at different concentrations. Blue, $20 \mathrm{wt} \%$, red, $10 \mathrm{wt} \%$ and black, $5 \mathrm{wt} \%$.

Depending on concentration, the $\mathrm{Co}^{2+}$ gels show a different change in storage and loss modulus with temperature. The damping factors of the $\mathrm{Co}^{2+}$ gels are approximately twenty five-fold higher at $60{ }^{\circ} \mathrm{C}$ than at $10{ }^{\circ} \mathrm{C}$. As illustrated in Figure 6.9, the storage modulus of the $10 \mathrm{wt} \%$ gel increases with temperature similarly as observed for the $\mathrm{Ni}^{2+}$ and $\mathrm{Fe}^{2+}$ gels. Contrary, $5 \mathrm{wt} \% \mathrm{Co}^{2+}$ gels show an initial increase in the storage modulus between 5 and $25^{\circ} \mathrm{C}$ followed by a decrease upon further heating. This is a clear indication that temperature affects the number of effective crosslinks in the gel.

This becomes even more pronounced for the $\mathrm{Zn}^{2+}$ gels. Increasing the temperature results in a gel to sol transition. The transition from a gel to sol occurs at approximately $25^{\circ} \mathrm{C}$ and is reversible upon cooling. Within the temperature range investigated, the storage modulus decreases with increasing temperatures, whereas the loss modulus 
increases up to the transition temperature and decreases upon further heating. This trend is witnessed for all $\mathrm{Zn}^{2+}$ gels at different concentrations. These results suggest that the increasing elastic modulus upon a temperature increase is counteracted by a decrease in elastic contribution due to dissociation of complexes. Thus, although temperature increases the modulus the equilibrium cross-link density decreases resulting in an overall decreasing modulus. Moreover, strain and frequency sweeps of the different complexes as presented in Figure 6.10 reveal that at a frequency of $\sim 1 \mathrm{~Hz}$ the $\mathrm{Zn}$ complexes are in the gel state and are stable up to high strains. The high modulus of the $\mathrm{Zn}$-complexes is possibly the result of efficient crosslinking. The frequency sweeps of the $\mathrm{Zn}$-complex show that at frequencies below $0.5 \mathrm{~Hz}$ the storage modulus is smaller than the loss modulus indicating a solution state. At low frequencies rearrangement by decomplexation and complexation of the Zn-complexes can occur. Above $0.5 \mathrm{~Hz}$ a gel state is observed and at these frequencies complexes have no time to rearrange leading to the formation of an elastic gel. These frequency sweeps indicate that the lability of Zn-complexes allows reorganization, which can lead to the formation of a homogeneous network [35]. At higher concentrations the mechanical properties of the $\mathrm{Zn}$ gels are increasing due to a higher crosslink density.
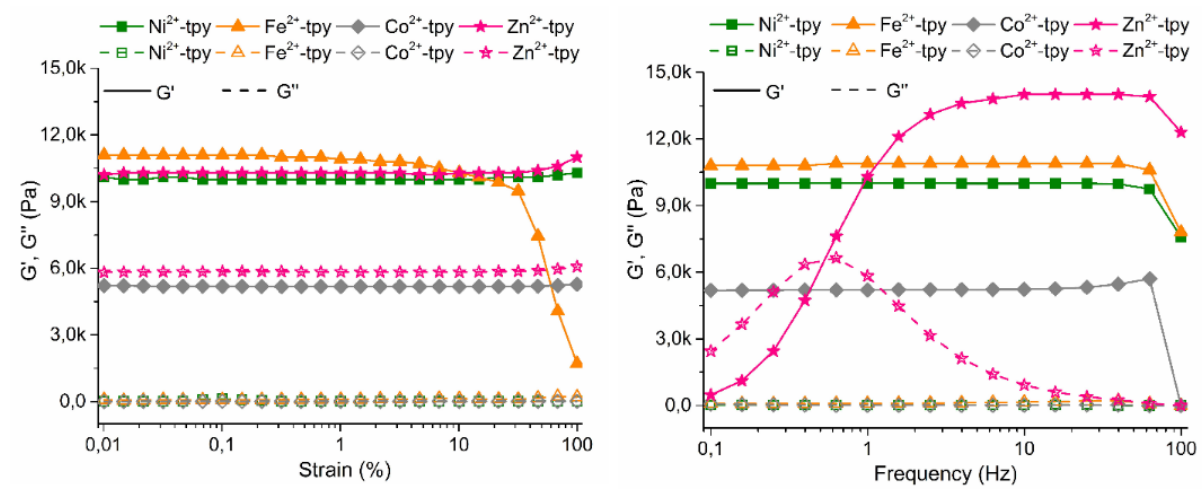

Figure 6.10 Strain (left) and frequency (right) sweeps of $\mathrm{Ni}^{2+}, \mathrm{Fe}^{2+}, \mathrm{Co}^{2+}$ or $\mathrm{Zn}^{2+}$ 8PEG(tpy $\left.{ }_{5.4}\right) \mathrm{OH}_{2.6}\left(\mathrm{M}^{2+}\right.$ :terpyridyl-groups $\left.=1: 2\right) 10 \mathrm{wt} \%$ hydrogels.

Interestingly, the gel to sol transition temperature of the $\mathrm{Zn}^{2+}$ gels does not change significantly with polymer content even though the modulus does increase at higher 
concentrations. The increasing cross-link density with polymer content resulting in an increased modulus is in accordance with the rubber elasticity theory. The temperature independent and reversible sol to gel transition suggests that more cross-links per unit volume are disrupted upon increasing temperatures in the higher polymer content gels compared to gels with a lower polymer content. At this moment we only can give a hypothesis to this phenomenon. The mechanical stability of hydrogels depends on the number of crosslinks formed and the inherent stability of the supramolecular crosslinks. Assuming the association rate of complex formation is very high, hydrogel properties likely depend on the dissociation rate constant of the complexes which has an Arrhenius type temperature dependency. This is reasonable as the formation of terpyridine complexes is an equilibrium process. Thus, at a certain temperature, the ratio between mono-complex and bis-complex (i.e. cross-links) per unit volume will be independent of the polymer content and free polymer chain ends will be formed more rapidly in respect to temperature in the higher polymer content gels compared to those with a lower polymer content. Therefore, polymer chain mobility will increase more rapidly with temperature, yielding a similar transition temperature for a gel with higher polymer content compared to a gel with lower polymer content.

\subsection{Conclusions}

The differences in dissociation rate constants of a supramolecularly crosslinked terpyridine end-group modified 8 arm PEG (8PEG-terpyridyl) with transition metal ions is reflected in nanoparticle formation at low concentrations and hydrogel properties at concentrations above $5 \mathrm{wt} \%$. Whereas $\mathrm{Ni}^{2+}$ or $\mathrm{Fe}^{2+}$ complexes at low concentrations afforded nanoparticles, $\mathrm{Co}^{2+}$ or $\mathrm{Zn}^{2+}$ complexes were mainly present as micellar type aggregates. Hydrogels formed by $8 \mathrm{PEG}\left(\right.$ tpy $\left._{5.4}\right) \mathrm{OH}_{2.6}$ with $\mathrm{Ni}^{2+}$ and $\mathrm{Fe}^{2+}$ were elastic and showed minor changes with temperature between 5 and $60{ }^{\circ} \mathrm{C}$. Due to the low kinetic stability of the $\mathrm{Co}^{2+}$ and $\mathrm{Zn}^{2+}$ complexes, the loss modulus of the hydrogels is largely influenced by temperature and led for the $\mathrm{Zn}^{2+}$ complexes to a reversible sol gel transition at $25^{\circ} \mathrm{C}$. Importantly, the sol-gel transition temperature of the $\mathrm{Zn}^{2+}$ complexes was independent of polymer content. 


\section{Reference}

[1] Aleman, J.; Chadwick, A. V.; He, J.; Hess, M.; Horie, K.; Jones, R. G.; Kratochvil, P.; Meisel, I.; Mita, I.; Moad, G.; Penczek, S.; Stepto, R. F. T., Definitions of terms relating to the structure and processing of sols, gels, networks, and inorganic-organic hybrid materials (IUPAC Recommendations 2007). Pure Appl. Chem. 2007, 79 (10), 1801-1827.

[2] Atwood, J. W. S. a. J. L. Supramolecular Chemistry; 2nd ed.; John Wiley \& Sons, Ltd.: United Kingdom, 2009.

[3] Hoffman, A. S., Hydrogels for biomedical applications. Adv. Drug Del. Rev. 2002, $54(1), 3-12$.

[4] Drury, J. L.; Mooney, D. J., Hydrogels for tissue engineering: scaffold design variables and applications. Biomaterials 2003, 24 (24), 4337-4351.

[5] Dobratz, E. J.; Kim, S. W.; Voglewede, A.; Park, S. S., Injectable Cartilage Using Alginate and Human Chondrocytes. Arch. Facial Plast. Surg. 2009, 11 (1), 40-47.

[6] Tang, Y. J.; Sun, J.; Fan, H. S.; Zhang, X. D., An improved complex gel of modified gellan gum and carboxymethyl chitosan for chondrocytes encapsulation. Carbohydr. Polym. 2012, 88 (1), 46-53.

[7] Suh, J. K. F.; Matthew, H. W. T., Application of chitosan-based polysaccharide biomaterials in cartilage tissue engineering: a review. Biomaterials 2000, 21 (24), 25892598.

[8] Goshe, A. J.; Crowley, J. D.; Bosnich, B., Supramolecular recognition: Use of cofacially disposed bis-terpyridyl square-planar complexes in self-assembly and molecular recognition. Helv. Chim. Acta 2001, 84 (10), 2971-2985.

[9] Brassinne, J.; Fustin, C. A.; Gohy, J. F., Polymer gels constructed through metalligand coordination. J. Inorg. Organomet P. 2013, 23 (1), 24-40.

[10] de Greef, T. F. A.; Meijer, E. W., Materials science - Supramolecular polymers. Nature 2008, 453 (7192), 171-173.

[11] Weng, W. G.; Beck, J. B.; Jamieson, A. M.; Rowan, S. J., Understanding the mechanism of gelation and stimuli-responsive nature of a class of metallosupramolecular gels. J. Am. Chem. Soc. 2006, 128 (35), 11663-11672. 
[12] Jones, C. J. D- and F-block Chemistry; 1st ed.; Royal Society of Chemistry: Cambridge, 2002.

[13] Albrecht, M., Supramolecular chemistry - general principles and selected examples from anion recognition and metallosupramolecular chemistry. Naturwissenschaften 2007, 94 (12), 951-966.

[14] König, B. Vorlesung Supramolekulare Chemie, Organische Chemie, 2009.

[15] Constable, E. C., 2,2':6',2"-terpyridines: From chemical obscurity to common supramolecular motifs. Chem. Soc. Rev. 2007, 36 (2), 246-253.

[16] Benniston, A. C.; Harriman, A.; Lawrie, D. J.; Mehrabi, M.; Russell, O. D., A ditopic receptor for cation binding and facilitated transport through a supported liquid membrane. Inorg. Chim. Acta 2005, 358 (12), 3483-3490.

[17] Constable, E. C.; Healy, J.; Drew, M. G. B., The crystal and molecular-structure of bis(2,2'-6',2"-terpyridine)magnesium perchlorate monohydrate. Polyhedron 1991, 10 (16), 1883-1887.

[18] Satterfield, M.; Brodbelt, J. S., Relative binding energies of gas-phase pyridyl ligand/metal complexes by energy-variable collisionally activated dissociation in a quadrupole ion trap. Inorg. Chem. 2001, 40 (21), 5393-5400.

[19] Kosbar, L.; Srinivasan, C.; Afzali, A.; Graham, T.; Copel, M.; Krusin-Elbaum, L., Self-assembled multilayers of transition-metal-terpyridinyl complexes; Formation, and characterization. Langmuir 2006, 22 (18), 7631-7638.

[20] Shunmugam, R.; Gabriel, G. J.; Aamer, K. A.; Tew, G. N., Metal-LigandContaining Polymers: Terpyridine as the Supramolecular Unit. Macromol. Rapid Commun. 2010, 31 (9-10), 784-793.

[21] Steed, J. W., Atwood, J.L. In Supramolecular Chemistry, ; 2 ed.; John Wiley \& Sons, Ltd.: 2009, p 27-36.

[22] Holyer, R. H.; Hubbard, C. D.; Kettle, S. F. A.; Wilkins, R. G., The Kinetics of Replacement Reactions of Complexes of the Transition Metals with 2,2',2"-Terpyridine. Inorg. Chem. 1966, 5 (4), 622-625.

[23] Henderson, I. M. Thesis, University of Massachusetts Amherst: Amherst, 2012.

[24] Whittell, G. R.; Hager, M. D.; Schubert, U. S.; Manners, I., Functional soft materials from metallopolymers and metallosupramolecular polymers. Nat Mater 2011, $10(3), 176-188$. 
[25] Lohmeijer, B. G. G.; Schubert, U. S., Playing LEGO with macromolecules: Design, synthesis, and self-organization with metal complexes. J. Polym. Sci., Part A: Polym. Chem. 2003, 41 (10), 1413-1427.

[26] Schmatloch, S.; Schubert, U. S., Engineering with metallo-supramolecular polymers: Linear coordination polymers and networks. Macromol Symp. 2003, 199, 483-497.

[27] Chiper, M.; Hoeppener, S.; Schubert, U. S.; Fustin, C. A.; Gohy, J. F., SelfAssembly Behavior of Bis(terpyridine) and Metallo-bis(terpyridine) Pluronics in Dilute Aqueous Solutions. Macromol. Chem. Phys. 2010, 211 (21), 2323-2330.

[28] Jochum, F. D.; Brassinne, J.; Fustin, C. A.; Gohy, J. F., Metallo-supramolecular hydrogels based on copolymers bearing terpyridine side-chain ligands. Soft Matter 2013, 9 (7), 2314-2320.

[29] Kimura, M.; Nakagawa, Y.; Adachi, N.; Tatewaki, Y.; Fukawa, T.; Shirai, H., Metallosupramolecular Gels Made of Four-armed Poly(ethylene glycol)s Having Terpyridine Termini. Chem. Lett. 2009, 38 (4), 382-383.

[30] Asoh, T.-A.; Yoshitake, H.; Takano, Y.; Kikuchi, A., Fabrication of self-healable hydrogels through sol-gel transition in metallo-supramolecular aqueous solution by aeration. Macromol. Chem. Phys. 2013, 214 (22), 2534-2539.

[31] Ueki, T.; Takasaki, Y.; Bundo, K.; Ueno, T.; Sakai, T.; Akagi, Y.; Yoshida, R., Autonomous viscosity oscillation via metallo-supramolecular terpyridine chemistry of branched poly(ethylene glycol) driven by the Belousov-Zhabotinsky reaction. Soft Matter 2014, 10 (9), 1349-1355.

[32] Rossow, T.; Seiffert, S., Supramolecular polymer gels with potential modelnetwork structure. Polym Chem-UK 2014, 5 (8), 3018-3029.

[33] Henderson, I. M.; Hayward, R. C., Substituent effects on the stabilities of polymeric and small molecule bis-terpyridine complexes. Polym Chem-UK 2012, 3 (5), 1221-1230.

[34] Hogg, R.; Wilkins, R. G., Exchange studies of certain chelate compounds of the transitional metals. Part VIII. 2,2',2'-terpyridine complexes. J. Chem. Soc. 1962, (0), 341-350.

[35] Rossow, T.; Habicht, A.; Seiffert, S., Relaxation and Dynamics in Transient Polymer Model Networks. Macromolecules 2014, 47 (18), 6473-6482. 


\section{Appendix}

From the assumption that the bis(terpyridyl) complex dissociation is rate limiting it follows that the rate equation below applies for the exchange of $\mathrm{Fe}^{2+}$ by $\mathrm{Ni}^{2+}$.

$$
v=k_{-2, F e^{2+}\left[F e^{2+}(t p y)_{2}\right]}
$$

Rewriting the rate equation into a differential equation and separating the variables yields the following.

$$
\frac{1}{\left[F e^{2+}(t p y)_{2}\right]} d\left[F e^{2+}(t p y)_{2}\right]=-k_{-2, F e} d t
$$

Subsequent integration yields equation 1 as shown below.

$$
\begin{gathered}
\int_{\left[F e^{2+}(t p y)_{2}\right]_{0}}^{\left[\mathrm{Fe}^{2+}(t p y)_{2}\right]_{t}} \frac{1}{\left[F e^{2+}(t p y)_{2}\right]} d\left[\mathrm{Fe}^{2+}(t p y)_{2}\right]=-\int_{0}^{t} k_{-2, F e} d t \\
\ln \left(\frac{\left[F e^{2+}(t p y)_{2}\right]_{t}}{\left[F e^{2+}(t p y)_{2}\right]_{0}}\right)=-k_{-2, F e} t \\
\rightarrow \quad\left[F e^{2+}(t p y)_{2}\right]_{t}=\left[F e^{2+}(t p y)_{2}\right]_{0} e^{-k-2, F e} t
\end{gathered}
$$

Similarly, during exchange of $\mathrm{Co}^{2+}$ by $\mathrm{Fe}^{2+}$, the concentration of $\mathrm{Co}^{2+}$ bis(terpyridyl) complexes is given by the following equation (A).

$$
\left[\mathrm{Co}^{2+}(t p y)_{2}\right]_{t}=\left[\mathrm{Co}^{2+}(t p y)_{2}\right]_{0} e^{-k_{-2, C o} t}
$$

The concentration of the $\mathrm{Co}^{2+}$ bis(terpyridyl) complexes can be related to the concentration of $\mathrm{Fe}^{2+}$ bis(terpyridyl) complexes through the equations below.

$$
\begin{gathered}
{\left[\mathrm{Fe}^{2+}(\mathrm{tpy})_{2}\right]_{t}=\frac{1}{2}\left(\left[\mathrm{Co}^{2+}(t p y)_{2}\right]_{0}-\left[\mathrm{Co}^{2+}(t p y)_{2}\right]_{t}\right)} \\
{\left[\mathrm{Co}^{2+}(t p y)_{2}\right]_{t}=\left[\mathrm{Co}^{2+}(t p y)_{2}\right]_{0}-2\left[\mathrm{Fe}^{2+}(t p y)_{2}\right]_{t}}
\end{gathered}
$$

Substituting the latter equation in equation A yields the premier equation beneath. Subsequent rewriting results in equation 2 . 


$$
\left[\mathrm{Co}^{2+}(t p y)_{2}\right]_{0}-2\left[\mathrm{Fe}^{2+}(t p y)_{2}\right]_{t}=\left[\mathrm{Co}^{2+}(t p y)_{2}\right]_{0} e^{-k_{-2, C o} t}
$$

$\rightarrow \quad\left[\mathrm{Fe}^{2+}(\mathrm{tpy})_{2}\right]_{t}=\frac{1}{2}\left[\mathrm{Co}^{2+}(t p y)_{2}\right]_{0}\left(1-e^{-k_{-2, C o} t}\right)$ 


\section{Chapter 7}

\section{Kinetically stable metal ligand charge transfer complexes as crosslinks in nanogels/hydrogels: physical properties and cytotoxicity*}
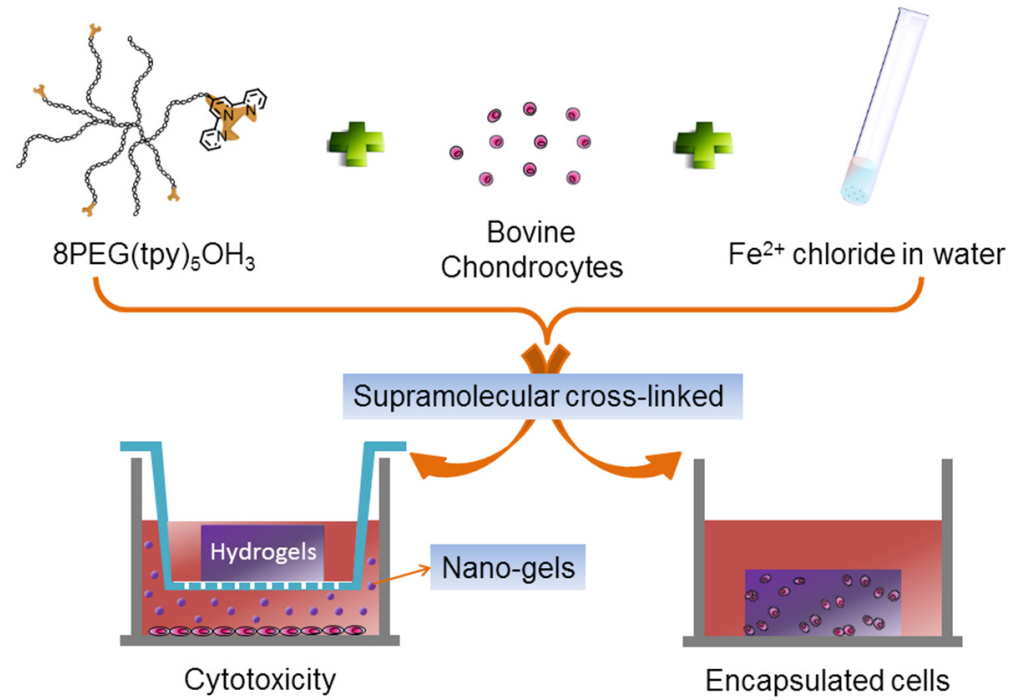

* This chapter has been published: Wang, R.; Both, S. K.; Geven, M.; Calucci, L.; Forte, C.; Dijkstra, P. J.; Karperien, M., Acta Biomater. 2015, 26, 136-144. 


\section{Abstract}

A terpyridine end-functionalized 8 arm poly(ethylene glycol) was prepared using the reaction of a 4'-aminopentanoxy substituted terpyridine with a p-nitrophenyl chloroformate activated 8PEG. Supramolecular complexation of the polymer terpyridine moieties by $\mathrm{Fe}^{2+}$ ions was investigated using NMR, UV-Vis and dynamic light scattering experiments. At low concentrations addition of $\mathrm{Fe}^{2+}$ ions to an aqueous solution of the polymer conjugate afforded nanogels with a single size distribution around $250 \mathrm{~nm}$. At concentrations above $3 \mathrm{wt} \%$, and at a 1:2 metal to ligand molar ratio, hydrogels were formed with increasing mechanical properties at increasing polymer concentrations. Using bovine chondrocytes, the biocompatibility and potential cytotoxicity of the polymer conjugate, nanogels and hydrogels were studied. Indirect contact of gels with chondrocytes using transwells revealed the absence of toxic components by leaching. A live-dead assay on chondrocytes encapsulated in the hydrogels indicated that the hydrogels are cytocompatible, revealing the potential use of these materials for biomedical and pharmaceutical applications. 


\subsection{Introduction}

Hydrogels, hydrophilic polymer networks that can retain large amounts of water, gained increasing interest in the past decades for their potential use in biomedical and pharmaceutical applications [1,4]. Due to their high water content, hydrogels do not evoke serious immune reactions and generally show good cytocompatibility. Hydrogels are commonly prepared by either chemical or physical cross-linking of polymer molecules. Chemical cross-linking generally affords mechanically stable polymer networks. Such hydrogels can be pre-formed before implantation or gelation can be performed in-situ by chemical or enzymatic reactions of polymer conjugates [1,2] [5,10]. Physical crosslinking occurs through supramolecular interactions like dipole interactions, ionic or biomimetic interactions, hydrogen bonding, $\pi-\pi$ stacking, stereocomplex formation or host-guest complexation [11,12]. As such physically cross-linked hydrogels do not require chemical reactions, conditions needed to form a gel generally can be mild. Moreover, the reversibility of these interactions provide material properties, like stimuliresponsiveness and self-healing, which are highly useful characteristics when applied in the biomedical and pharmaceutical fields.

The formation of host-guest complexes, as a method to induce physical cross-linking, can result in stable materials when multiple bases in a molecule donate electrons to a single Lewis acid. The thermodynamic stability of complexes not only depends on the multiple bonding interactions present, known as the chelate effect, but also on the preorganization of the ligands prior to complex formation $[13,14]$. In recent years it was shown that, although these interactions can be relatively strong, and depend on the transition metal ion used, the kinetic stability of the metal coordination bond can vary widely. The kinetic properties of coordination complexes are particularly of relevance for their possible exploitation, for example, as dynamic cross-links in hydrogels. Such stimuli-responsive gels are nowadays termed metallo supramolecular polymer gels (MSPGs) [15].

Several strategies may be applied to construct polymer gels using metal-ligand coordination, as recently reviewed by the group of Gohy [16]. The complexation of two $2,2^{\prime}: 6$ ',2"-terpyridine ligands with a single transition metal ion, as a method to induce cross-linking, was frequently studied to prepare MSPGs $[17,18]$. In particular, the use of 
multi-armed poly(ethylene glycol)s end-functionalized with terpyridine groups provides a versatile method to prepare hydrogels by cross-linking through transition metal ion complexation. Kimura et al. [19] showed that a terpyridine end-functionalized 4-arm PEG formed a hydrogel at a relatively high concentration of $20 \mathrm{wt} \%$ upon complexation with $\mathrm{Fe}^{2+}$ ions in water. This gel could be dissociated by the addition of ammonium hydroxide, as shown by the disappearance of the characteristic purple color of $\mathrm{Fe}^{2+}$-terpyridine complexes. When a terpyridine functionalized 3-arm PEG was complexed with $\mathrm{Co}^{2+}$ ions, oxidation to $\mathrm{Co}^{3+}$ by aeration led to the formation of a hydrogel. Reduction-oxidation cycles revealed the reversibility of the system [20]. A more elaborate study on 4-arm PEG end-functionalized with terpyridine groups through click chemistry was performed by Rossow and Seiffert [21-23]. They studied the network mechanical properties of gels formed by complex formation with $\mathrm{Zn}^{2+}, \mathrm{Co}^{2+}$ and $\mathrm{Mn}^{2+}$ ions. The complexation of $\mathrm{Co}^{2+}$ ions leads to network heterogeneity, which appears absent in the case of gels formed with $\mathrm{Zn}^{2+}$ ions. When complexed with $\mathrm{Ru}^{2+}$ ions, terpyridine end-functionalized 4- and 8 arm PEG afforded hydrogels. Rheological measurements revealed that the sol-gel transition occurred much more quickly in the 8 arm PEG than in the 4-arm PEG, but that the number of efficient cross-linking points participating in the gel network is almost the same in both systems [24]. Recently, multi-responsive supramolecular cross-linked polyglycerol hydrogels by hydrogen bonding and metal complexation were developed. The responsiveness allows degelling by a change in $\mathrm{pH}$ to reverse the cross-linking by hydrogen bonding. Metal chelators can be used to reverse the cross-linking by metal complexation. This class of hydrogels is regarded promising for use in life science studies [25]. In a recent study it was shown that 8 arm poly(ethylene glycol) partially functionalized with terpyridine groups formed hydrogels with the transition metal ions $\mathrm{Ni}^{2+}, \mathrm{Fe}^{2+}, \mathrm{Co}^{2+}$ and $\mathrm{Zn}^{2+}$ at concentrations above $5 \mathrm{wt} \%$. The $\mathrm{Ni}^{2+}$ and $\mathrm{Fe}^{2+}$ complexes appeared kinetically stable and showed minor changes in mechanical properties at temperatures between 5 and $60{ }^{\circ} \mathrm{C}$, whereas the $\mathrm{Co}^{2+}$ and $\mathrm{Zn}^{2+}$ complexes revealed a low kinetic stability. Interestingly, the reversible sol to gel transition temperature of the $\mathrm{Zn}^{2+}$ complexes was independent of polymer content [26].

Polymeric hydrogels based on terpyridine end-functionalized PEGs may have potential in biomedical and pharmaceutical applications as in-situ forming materials. Up to now the biological evaluation of this class of hydrogels has not been investigated. It 
may be envisaged that released metal ions may invoke a toxic effect or that decomplexed ligands will complex essential trace metal ions from the tissue. In this study, research was focused on a terpyridine end-functionalized 8 arm PEG either in the uncomplexed and complexed state with $\mathrm{Fe}^{2+}$ ions and the potential toxic effects were investigated in direct or indirect contact tests using chondrocytes. Nanogels and hydrogels were physically characterized and biologically evaluated through proliferation, viability and cytotoxicity assays of chondrocytes.

\subsection{Materials and methods}

\subsubsection{Materials}

Hydroxyl terminated 8 arm poly(ethylene glycol) ( $8 \mathrm{PEG}, \mathrm{M}_{\mathrm{n}, \mathrm{NMR}}=21400 \mathrm{~g} / \mathrm{mol}$ ) was purchased from Jenkem (Allen, Texas, USA) and purified before use by dissolution in dichloromethane and precipitation in cold diethyl ether. 4-arm PEG acrylate (4PEG-DA, Mw $20 \mathrm{kDa}$ ) was obtained from Laysan Bio (Arab, AL, USA). 4'-Chloro-2,2':6',2'”terpyridine (99\%), 5-amino-1-pentanol (95\%), pyridine (99.8\%), lithium chloride ( $\mathrm{LiCl}$, $\geq 98 \%$ ), potassium hydroxide ( $\mathrm{KOH}, \geq 85 \%$ ), 2-hydroxy-4'-(2-hydroxyethoxy)-2methylpropiophenone (Irgacure 2959), Iron(II)chloride (anhydrous, 99.99\%) and anhydrous dimethyl sulfoxide (DMSO, $\geq 99.9 \%$ ) were obtained from Aldrich-Sigma (Zwijndrecht, the Netherlands). $p$-Nitrophenyl chloroformate (PNC) was purchased from Fluka (Zwijndrecht, the Netherlands) and was purified by sublimation under vacuum at $90{ }^{\circ} \mathrm{C}$. $\mathrm{LiCl}$ was dried at $105^{\circ} \mathrm{C}$ in a vacuum oven before use. All solvents were from Biosolve (Valkenswaard, the Netherlands). All chemicals and solvents were used as received.

\subsubsection{Synthesis}

5-(2,2':6',2',-Terpyridine-4'-yloxy)-pentyl-1-amine (tpy-A): To a stirred suspension of powdered $\mathrm{KOH}(0.505 \mathrm{~g}, 9.02 \mathrm{mmol})$ in DMSO $(50 \mathrm{~mL}), 5$-amino-1-pentanol $(1.67 \mathrm{~g}$, $16.2 \mathrm{mmol}$ ) was added drop wise at $40^{\circ} \mathrm{C}$. After $20 \mathrm{~min} 4^{\prime}$-chloro-2,2':6',2'”-terpyridine was added $(1.00 \mathrm{~g}, 3.73 \mathrm{mmol})$ and the mixture was stirred for $2 \mathrm{~h}$ at $40{ }^{\circ} \mathrm{C}$ and then 
poured into deionized water $(500 \mathrm{~mL})$. The product was filtered, washed with deionized water and subsequently the crude product was recrystallized from hot ethanol. The pale yellow solid was dried under vacuum (yield $85 \%$ ). ${ }^{1} \mathrm{H}$ NMR (400 $\mathrm{MHz}, \mathrm{CDCl}_{3}$ ): $\delta=1.52$ $1.57\left(\mathrm{~m}, 4 \mathrm{H}, \mathrm{OCH}_{2} \mathrm{CH}_{2} \mathrm{CH}_{2} \mathrm{CH}_{2} \mathrm{CH}_{2} \mathrm{NH}_{2}\right.$ ), 1.87 (t, $\left.2 \mathrm{H}, \mathrm{OCH}_{2} \mathrm{CH}_{2} \mathrm{CH}_{2}\right), 2.73$ (t, $2 \mathrm{H}$, $\mathrm{CH}_{2} \mathrm{NH}_{2}$ ), 4.23 (t, $2 \mathrm{H}, \mathrm{OCH}_{2} \mathrm{CH}_{2}$ ), 7.32 (m, $2 \mathrm{H}$, terpyridine), 7.84 (dd, $2 \mathrm{H}$, terpyridyl), 8.03 (s, $2 \mathrm{H}$, terpyridine), $8.60\left(\mathrm{~d}, 2 \mathrm{H}\right.$, terpyridine), $8.69\left(\mathrm{~d}, 2 \mathrm{H}\right.$, terpyridine) ${ }^{13} \mathrm{C}$ NMR (400 MHz, $\left.\mathrm{CDCl}_{3}\right): \delta=23.4\left(\mathrm{CH}_{2} \mathrm{CH}_{2} \underline{\mathrm{CH}_{2}} \mathrm{CH}_{2} \mathrm{CH}_{2}\right), 28.9\left(\mathrm{CH}_{2} \underline{\mathrm{CH}_{2}} \mathrm{CH}_{2} \mathrm{CH}_{2} \mathrm{CH}_{2}\right), 33.6$

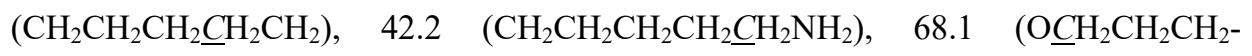
$\mathrm{CH}_{2} \mathrm{CH}_{2}$ ), 107.4, 121.3, 123.8, 136.8, 149.0, 156.2, 157.1, 167.3 (terpyridine).

8PEG(PNC) $)_{5} \mathbf{O H}_{3}$ : To a solution of 8 PEG $(2.00 \mathrm{~g}, 0.093 \mathrm{mmol})$ and lithium chloride $(1.00 \mathrm{~g}, 23.8 \mathrm{mmol})$ in $100 \mathrm{~mL}$ of DMF were added $p$-nitrophenol chloroformate $(0.804$ $\mathrm{g}, 4.00 \mathrm{mmol})$ and pyridine $(0.978 \mathrm{~g}, 12.4 \mathrm{mmol})$ at $0{ }^{\circ} \mathrm{C}$. The polymer solution was stirred at $0{ }^{\circ} \mathrm{C}$ for $1 \mathrm{~h}$ and then poured into $500 \mathrm{~mL}$ of cold ethanol/diethyl ether $(4 / 1 \mathrm{v} / \mathrm{v})$. The precipitate was filtered and washed with cold ethanol and diethyl ether, and then dried in a vacuum oven, yielding $8 \mathrm{PEG}(\mathrm{PNC})_{5} \mathrm{OH}_{3}$ as a white solid (yield 94\%). ${ }^{1} \mathrm{H}$ NMR (400 $\mathrm{MHz}, \mathrm{CDCl}_{3}$ ): $\delta=3.63$ (PEG protons), 4.34 ( $\mathrm{m}, \mathrm{OCH}_{2} \mathrm{CH}_{2} \mathrm{OCO}$ ), 7.37-7.40 and 8.268.28 (m, aromatic protons).

8PEG(tpy) $)_{5} \mathrm{OH}_{3}$ : To a solution of $8 \mathrm{PEG}(\mathrm{PNC})_{5} \mathrm{OH}_{3}(2.00 \mathrm{~g}, 0.092 \mathrm{mmol})$ in $20 \mathrm{~mL}$ of dichloromethane was added 5-(2,2':6',2''-terpyridine-4'-yloxy)-pentyl-1-amine (0.272 $\mathrm{g}, 0.814 \mathrm{mmol}$ ) at room temperature. The solution was stirred under a $\mathrm{N}_{2}$ atmosphere for $24 \mathrm{~h}$. The product was precipitated in cold diethyl ether, washed with cold ethanol and then dried under vacuum for 1 day to give $8 \mathrm{PEG}(\text { tpy) })_{5} \mathrm{OH}_{3}$ (yield 84\%). ${ }^{1} \mathrm{H}$ NMR (400 $\left.\mathrm{MHz}, \quad \mathrm{CDCl}_{3}\right): \delta=1.52-1.57 \quad\left(\mathrm{~m}, \quad \mathrm{NHCH}_{2} \mathrm{CH}_{2} \mathrm{CH}_{2} \mathrm{CH}_{2} \mathrm{CH}_{2} \mathrm{O}\right), 1.87 \quad(\mathrm{t}$, $\mathrm{NHCH}_{2} \mathrm{CH}_{2} \mathrm{CH}_{2} \mathrm{CH}_{2} \mathrm{CH}_{2} \mathrm{O}$ ), 3.21 (m, $\mathrm{CH}_{2} \mathrm{NH}$ ), 3.63 ( $\mathrm{PEG}$ protons), 4.23 (t, $\mathrm{CH}_{2} \mathrm{O}$ ), 5.00 (s, $\mathrm{NH}), 7.32$ (m, terpyridine), 7.84 (dd, terpyridine), 8.03 (s, terpyridine), 8.60 (d, terpyridine), 8.69 (d, terpyridine).

\subsubsection{Analysis}

NMR spectroscopy: ${ }^{1} \mathrm{H}$ and ${ }^{13} \mathrm{C}$ NMR spectra on samples dissolved in $\mathrm{CDCl}_{3}$ were recorded on a Bruker Ascend ${ }^{\mathrm{TM}}$ III 400 NMR (Nanobay) Spectrometer working at 400.13 and $100.62 \mathrm{MHz}$ for ${ }^{1} \mathrm{H}$ and ${ }^{13} \mathrm{C}$, respectively. The chemical shifts were calibrated against 
residual solvent signals. Peak multiplicity was denoted as s (singlet), d (doublet), dd (double doublet), $\mathrm{t}$ (triplet), q (quartet), and $\mathrm{m}$ (multiplet).

${ }^{1} \mathrm{H}$ NMR experiments on samples dissolved in water were carried out on a Bruker AMX-300 WB spectrometer working at $300.13 \mathrm{MHz}$ for proton, using a $5 \mathrm{~mm}$ probe head, a $90^{\circ}$ pulse of $6 \mu \mathrm{s}$ and a recycle delay of $5 \mathrm{~s}$, and acquiring from 40 to 200 scans depending on sample concentration. Samples containing 1, 2, 5, 8 and 10 wt \% of 8PEG(tpy $)_{5} \mathrm{OH}_{3}$, were prepared by dissolving the appropriate amounts of polymer in a $\mathrm{H}_{2} \mathrm{O} / \mathrm{D}_{2} \mathrm{O}$ mixture (10 vol\% $\mathrm{D}_{2} \mathrm{O} 99.98 \% \mathrm{D}$, Eurisotop). ${ }^{1} \mathrm{H}$ NMR spectra were recorded on all samples at $37^{\circ} \mathrm{C}$; the $10 \mathrm{wt} \%$ sample experiments were also performed at different temperatures from 20 to $70{ }^{\circ} \mathrm{C}$ in 5 degree steps. The signals of $8 \mathrm{PEG}(\text { tpy })_{5} \mathrm{OH}_{3}$ in $\mathrm{D}_{2} \mathrm{O}$ were assigned with the aid of a ${ }^{1} \mathrm{H}$ COSY experiment. Chemical shifts are given in ppm with respect to 3-(trimethylsilyl)-1-propanesulfonic acid sodium salt in $\mathrm{D}_{2} \mathrm{O}$ as external standard.

${ }^{1} \mathrm{H}$ NMR titration experiments were performed at $37^{\circ} \mathrm{C}$ by reacting $500 \mu \mathrm{l}$ of a $10 \mathrm{mg}$ $\mathrm{mL}^{-1}$ solution of $8 \mathrm{PEG}(\text { tpy })_{5} \mathrm{OH}_{3}$ in $\mathrm{D}_{2} \mathrm{O}$ with successive $5 \mu$ aliquots of a $1.2510^{-2} \mathrm{M}$ solution of $\mathrm{FeCl}_{2}$ in $\mathrm{D}_{2} \mathrm{O}$. Solutions were prepared using $\mathrm{D}_{2} \mathrm{O}$ saturated with argon and the mixtures were vortexed for at least $5 \mathrm{~min}$ after each addition.

UV-Vis spectroscopy: UV-Vis absorption spectra were recorded at room temperature on a Cary $300 \mathrm{UV}$-Vis using $1 \mathrm{~cm}$ quartz cuvettes. An aqueous solution of $8 \mathrm{PEG}(\text { tpy })_{5} \mathrm{OH}_{3}$ $\left(0.5 \mathrm{mg} \mathrm{mL}^{-1}, 2.15 \times 10^{-4} \mathrm{mmol}\right)$ was prepared. A solution of $\mathrm{FeCl}_{2}$ in water was prepared at a suitable concentration that minimizes dilution upon titration. During the titration experiment, a selected amount was added stepwise and the resulting solution was stirred for at least $5 \mathrm{~min}$ to allow equilibration. The formation of the terpyridine- $\mathrm{Fe}^{2+}$ complex was monitored by recording spectra between 200 and $800 \mathrm{~nm}$. The data were corrected for dilution upon titration. The absorption and $\lambda(\mathrm{nm})$ for the $8 \mathrm{PEG}(\text { tpy })_{5} \mathrm{OH}_{3} \cdot \mathrm{Fe}^{2+}$ complex were: $2.33(315 \mathrm{~nm}), 0.32(360 \mathrm{~nm})$ and $0.74(558 \mathrm{~nm})$.

Dynamic light scattering: DLS experiments were performed on $0.5 \mathrm{wt} \%$ solutions using a Malvern zetasizer 4000 (Malvern Corp., Malvern, UK) at a laser wavelength of $633 \mathrm{~nm}$ and a scattering angle of $173^{\circ}$. The micelle or aggregate size of copolymers in deionized water was determined as a function of metal ion concentration at $25^{\circ} \mathrm{C}$. All suspensions were allowed to equilibrate for $15 \mathrm{~min}$ before each measurement. 
Scanning electron microscopy: The size of the nanogels was determined with a HRSEM-Zeiss scanning electron microscope (SEM) equipped with NORAN EDS and WDS with a maximum and minimum accelerating voltage of $30 \mathrm{kV}$ and $0.2 \mathrm{kV}$, respectively, and a field-emission.

Rheology: Oscillatory rheology experiments were performed on $8 \mathrm{PEG}(\text { tpy })_{5} \mathrm{OH}_{3} \cdot \mathrm{Fe}^{2+}$ hydrogels with concentrations of 5,8,10, and $20 \mathrm{wt} \%$ prepared by dissolution of $8 \mathrm{PEG}(\text { tpy })_{5} \mathrm{OH}_{3}$ in water and subsequent addition of an appropriate amount of a $\mathrm{FeCl}_{2}$ solution $\left(\mathrm{Fe}^{2+}\right.$ :terpyridine molar ratio equal to $\left.1: 2\right)$. The storage $\left(\mathrm{G}^{\prime}\right)$ and loss $\left(\mathrm{G}^{\prime \prime}\right)$ modulus of the hydrogels were monitored for $30 \mathrm{~min}$ at $25^{\circ} \mathrm{C}$ on a Physica MCR 301 rheometer (Anton Paar) using a flat plate geometry ( $25 \mathrm{~mm}$ diameter, $0.3 \mathrm{~mm}$ gap) in oscillating mode. The hydrogels (approximately $300 \mu \mathrm{L}$ ) were placed on the rheometer plate and a solvent trap was used over the geometry to prevent water evaporation. A preshear was applied for $10 \mathrm{~s}$, after which the polymer solution was allowed to equilibrate for $10 \mathrm{~min}$. The mechanical properties were monitored by measuring the storage modulus $\mathrm{G}^{\prime}$ and the loss modulus $\mathrm{G}$ " at $25^{\circ} \mathrm{C}$. A frequency $\omega$ of $1 \mathrm{~Hz}$ and a strain $\gamma$ of $1 \%$ were applied to minimize the influence of deformation on the hydrogels. Then, a strain sweep was recorded at constant frequency $(\omega=1 \mathrm{~Hz} ; \gamma=0.1-10 \%)$ and after $10 \mathrm{~min}$ equilibration, a frequency sweep was recorded at constant strain amplitude $(\gamma=1 \% ; \omega=$ $0.1-100 \mathrm{~Hz})$ at $25^{\circ} \mathrm{C}$.

\subsubsection{In vitro cytotoxicity}

Direct cytotoxicity tests: Chondrocytes from bovine cartilage were isolated as previously described and cultured in chondrocyte expansion medium composed of DMEM (Invitrogen) with $10 \%$ fetal bovine serum (FBS, Sigma), 1\% penicillin/streptomycin (Invitrogen), $0.01 \mathrm{M}$ nonessential amino acids (NEAA, Sigma), $0.02 \mathrm{mM}$ ascorbic acid (ASAP, Sigma) and $0.04 \mathrm{mM}$ L-proline (Sigma) at $37^{\circ} \mathrm{C}$ in a humidified atmosphere $(95$ $\%$ air and $5 \% \mathrm{CO}_{2}$ ) [27]. The chondrocytes (passage 2) were seeded in 24-well plates at a density of 2500 cells $/ \mathrm{cm}^{2}$ and cultured in chondrocyte expansion medium overnight. Solutions of $8 \mathrm{PEG}\left(\mathrm{tpy}_{5}\right)_{5} \mathrm{OH}_{3}$ with polymer concentrations of 250 or $500 \mu \mathrm{g} \mathrm{mL}-1, \mathrm{FeCl}_{2}$ solutions with concentrations of 3.83 or $7.66 \mu \mathrm{g} \mathrm{mL} \mathrm{L}^{-1}$ and $8 \mathrm{PEG}(\text { tpy })_{5} \mathrm{OH}_{3} \cdot \mathrm{Fe}^{2+}$ $\left(\mathrm{Fe}^{2+}\right.$ :terpyridine molar ratio of $\left.1: 2\right)$ nanogel suspensions of 250 or $500 \mu \mathrm{g} \mathrm{mL}^{-1}$ in 
chondrocyte expansion medium were sterilized using a $0.22 \mu \mathrm{m}$ filter. To the wells, $1 \mathrm{~mL}$ of the above given solutions or suspensions was added. The well plates were placed in an incubator $\left(37^{\circ} \mathrm{C}, 5 \% \mathrm{CO}_{2}\right)$ and the medium was replaced every 2 or 3 days. Viability of the cells was determined at days 1, 4 and 7 by a PrestoBlue assay (Invitrogen). Quantification of total DNA was done by a CyQUANT DNA assay (Molecular Probe, Invitrogen) using a fluorescent plate reader (Perkin-Elmer).

Indirect cytotoxicity tests: The cytotoxicity of potential leaching compounds from the hydrogels on chondrocytes was tested using a transwells system. The $8 \mathrm{PEG}(\text { tpy })_{5} \mathrm{OH}_{3} \cdot \mathrm{Fe}^{2+}$ hydrogels were prepared at a concentration of $8 \mathrm{wt} \%$. Chondrocytes from bovine cartilage (passage 2) were seeded in 24-well plates at a density of 2500 cells $/ \mathrm{cm}^{2}$ and left in chondrocyte expansion medium overnight. Thereafter the inserts, each containing $200 \mathrm{mg}$ of hydrogels were placed in the wells. Inserts with $8 \mathrm{wt} \%$ 4PEGDA hydrogels or empty transwells were used as controls. The medium was replaced every 2 or 3 days. Viability of the cells was determined at days 1, 4 and 7 by PrestoBlue assay and quantification of total DNA content was done by a CyQUANT DNA assays.

4PEG-DA gel: An $8 \mathrm{wt} \%$ solution of 4-arm PEG acrylate in $200 \mu \mathrm{L}$ of phosphate buffered saline (PBS, pH 7.4) containing $0.05 \mathrm{wt} \%$ Irgacure 2959 as initiator was prepared. The solution was placed in a UV box and polymerization was performed at 365 $\mathrm{nm}, 10 \mathrm{~mW} / \mathrm{cm}^{2}$ (UV lamp, Ultra-Lum, Inc.) for $30 \mathrm{~min}$ to yield hydrogels. Hydrogels were sterilized by incubating in ethanol overnight and washed three times with PBS for one hour.

Chondrocyte-hydrogel constructs: Hydrogels incorporating bovine chondrocytes were prepared under sterile conditions by mixing $8 \mathrm{PEG}(\text { tpy })_{5} \mathrm{OH}_{3} / \mathrm{cell}$ suspensions with a freshly made $\mathrm{FeCl}_{2}$ stock solution. Stock solutions of $\mathrm{FeCl}_{2}$ in cell culture water were diluted using medium and sterilized using a filter with a pore size of $0.22 \mu \mathrm{m}$. In a typical example, to incorporate chondrocytes in the hydrogels, $70 \mu \mathrm{L}$ of the chondrocytes/medium suspension was mixed with $10 \mathrm{mg}$ of $8 \mathrm{PEG}(\text { tpy })_{5} \mathrm{OH}_{3}$ and subsequently $30 \mu \mathrm{L}$ of a $0.49 \mathrm{wt} \% \mathrm{FeCl}_{2}$ stock solution was added to give a $10 \mathrm{wt} \%$ $8 \mathrm{PEG}(\text { tpy })_{5} \mathrm{OH}_{3} \cdot \mathrm{Fe}^{2+}$ complex hydrogel; the cell density was $3 \times 10^{6}$ cells $/ \mathrm{mL}$. In a similar way the $4 \mathrm{wt} \%$ and $8 \mathrm{wt} \% 8 \mathrm{PEG}(\text { tpy })_{5} \mathrm{OH}_{3} \cdot \mathrm{Fe}^{2+}$ hydrogels were prepared and an $0.5 \mathrm{wt} \%$ agarose gel was used as a positive control. The gels were prepared in round-bottom 96well plates and transferred to 24-well plates and subsequently incubated in chondrocyte 
expansion medium. The medium was replaced every 2 or 3 days. Viability of the cells was determined at days 1, 4 and 7 by a Live-Dead assay (Invitrogen).

PrestoBlue assay: The metabolic activity of chondrocytes in the conditioned environments (see section direct cytotoxicity tests), was determined using the PrestoBlue kit (Invitrogen) according the manufacturer's instructions [28]. The cells were first washed with PBS and $10 \mu \mathrm{L}$ of PrestoBlue reagent in $90 \mu \mathrm{L}$ of chondrocyte medium was added. Following an incubation time of $30 \mathrm{~min}$ at $37^{\circ} \mathrm{C}$, the PrestoBlue medium was transferred to a 96-well plate and the fluorescence of each well was measured using a microplate reader (Victor) with excitation at 560/10 nm and emission at 590/10 nm. Data are presented as average $\pm \mathrm{SD}(\mathrm{n}=3)$. Data were also statistically analyzed by using GraphPad Prism software.CyQUANT DNA assay: The DNA content of chondrocytes present at days 1, 4 and 7 in culture was quantitated by using a fluorimetric assay of cell lysates (CyQUANT Cell Proliferation Assay Kit, Invitrogen). In order to lyse the cells, the samples were put in a freezer overnight, thereafter thawed and sonicated for $30 \mathrm{~min}$. To each sample, $650 \mu \mathrm{L}$ of CyQUANT reagent was added. Samples were fully covered with foil to protect from light and incubated at room temperature for $1 \mathrm{~h}$. The fluorescence was measured with excitation at $480 / 10 \mathrm{~nm}$ and emission at 520/10 $\mathrm{nm}$ using a microplate reader (Victor) according the manufacturer's instructions. Cell numbers were determined by comparing the average fluorescence of sample to cell standard curve.

Live-Dead assay: The viability of the chondrocytes (passage 2-3) encapsulated in the hydrogels was determined using a Live-Dead assay. At days 1, 7 and 14, the hydrogel constructs were rinsed with PBS and stained with calcein AM/ethidium homodimer, according the manufacturer's instructions. Agarose hydrogels $(0.5 \mathrm{wt} \%)$ with a similar chondrocyte density were used as a control. Hydrogel/cell constructs were visualized using fluorescence microscopy (Nikon). As a result living cells stain green and the nuclei of dead cells red.

Statistical analysis: Data $(n=3)$ are presented as mean \pm standard deviation (SD). Statistical differences among three or more groups were analyzed using the One-way Analysis of Variance (ANOVA) with Turkey's post-hoc analysis. Statistical significance was set to a $p$ value $\leq 0.05$. 


\subsection{Results and Discussion}

\subsubsection{Synthesis of terpyridine end functionalized 8 arm poly(ethylene glycol)}

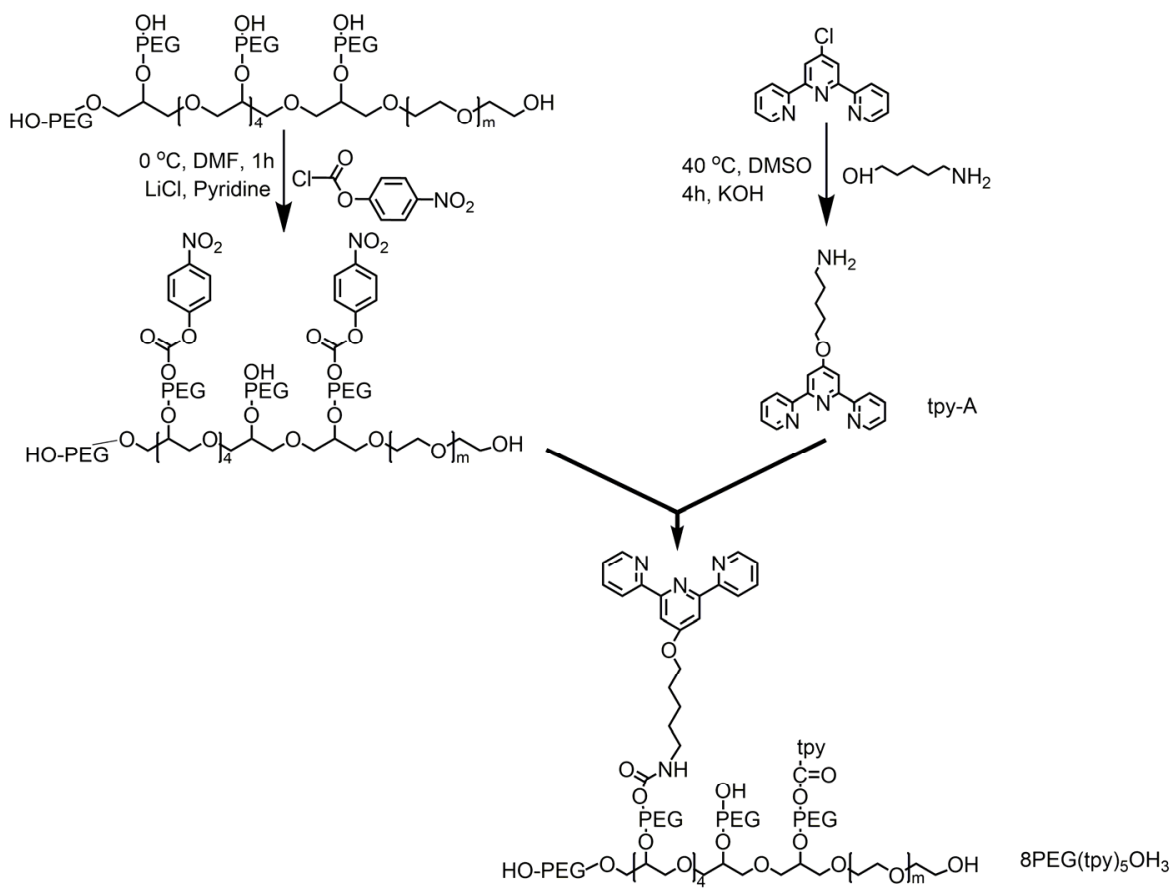

Scheme 7.1 Synthesis of the terpyridine end-functionalized 8PEG.

The synthesis of a partially terpyridine end-functionalized 8 arm poly(ethylene glycol) (8PEG) was performed by first reacting the hydroxyl end-groups of the 8PEG with pnitrophenyl chloroformate. Full conversion of the hydroxyl groups, even at a large excess of the p-nitrophenyl chloroformate, could not be accomplished. This result is similar as observed previously in the end-group modification of an 8 arm PEG with terpyridine groups [26]. However, in a reproducible way five of the hydroxyl groups could be converted to p-nitrophenyl carbonate (PNC) groups as determined from the relative intensities of the aromatic and PEG proton signals in the ${ }^{1} \mathrm{H}$ NMR spectra. To couple terpyridine groups to the $8 \mathrm{PEG}(\mathrm{PNC})_{5} \mathrm{OH}_{3}$, a 4'-aminopentanoxy substituted terpyridine 
(tpy-A) was prepared from the corresponding 4'-chloro substituted terpyridine [29]. Reaction of the $8 \mathrm{PEG}(\mathrm{PNC})_{5} \mathrm{OH}_{3}$ with an excess of tpy-A monomer afforded the 8 arm PEG linked with 5 terpyridine and 3 hydroxyl end groups $\left(8 \mathrm{PEG}(\mathrm{tpy})_{5} \mathrm{OH}_{3}\right)$ per polymer molecule (Scheme 7.1). The successful coupling of tpy-A was assessed by ${ }^{1} \mathrm{H}$ NMR spectral data, which showed the signals ascribable to the carbamate protons at $5.0 \mathrm{ppm}$ and terpyridine protons at 7.32-8.69 ppm (Figure 7.1). The number of terpyridine groups per polymer molecule was determined from the relative integrals of terpyridine and poly(ethylene glycol) peaks.

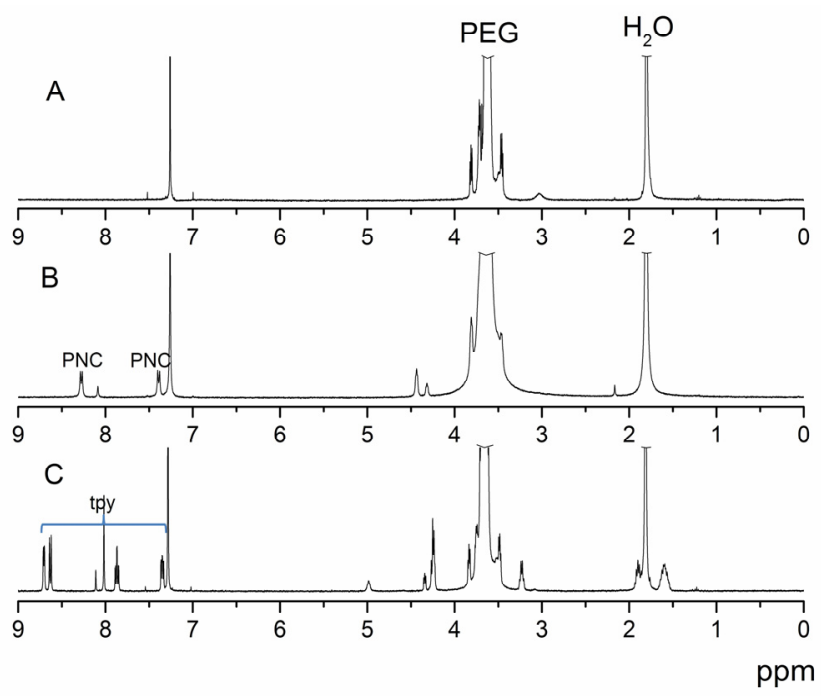

Figure 7.1 ${ }^{1} \mathrm{H}$ NMR spectra $\left(400 \mathrm{MHz}, \mathrm{CDCl}_{3}\right)$ of $8 \mathrm{PEG}(\mathrm{A}), 8 \mathrm{PEG}(\mathrm{PNC})_{5} \mathrm{OH}_{3}(\mathrm{~B})$ and 8PEG(tpy) ${ }_{5} \mathrm{OH}_{3}(\mathrm{C})$.

\subsubsection{Complexation behavior}

The complexation of the polymer terpyridine groups with $\mathrm{Fe}^{2+}$ ions in water was investigated by UV-Vis and ${ }^{1} \mathrm{H}$ NMR titration experiments. The UV-Vis spectra of a 8PEG(tpy $)_{5} \mathrm{OH}_{3}$ aqueous solution upon the stepwise addition of $\mathrm{FeCl}_{2}$ showed the metalto-ligand charge-transfer (MLCT) band at $556 \mathrm{~nm}$ (Figure 7.2) characteristic of $\mathrm{Fe}^{2+}$ :terpyridine complexes. The maximum absorption observed for the MCLT band at a 
1:2 $\mathrm{Fe}^{2+}$ :terpyridine molar ratio shows that likely only complexes where two terpyridine moieties are coordinated to a metal ion are formed [30].

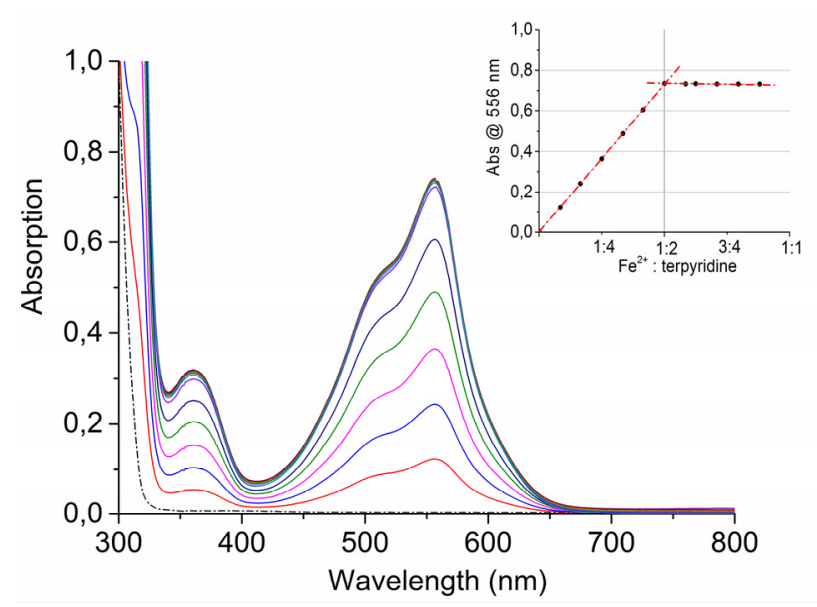

Figure 7.2 UV-Vis absorption spectra recorded for the titration of $8 \mathrm{PEG}\left(\mathrm{tpy}_{5}\right)_{5} \mathrm{OH}_{3}$ with $\mathrm{FeCl}_{2}$ in water, corrected for the absorption of $8 \mathrm{PEG}(\text { tpy })_{5} \mathrm{OH}_{3}$ (dashed line). In the inset absorption values at $556 \mathrm{~nm}$ are presented. Data were corrected for dilution upon titration.

The complex formation was further investigated using ${ }^{1} \mathrm{H}$ NMR titration experiments. Upon complexation, shifts of the signals relative to terpyridine protons and to protons of terminal methylene groups of the PEG chains (Figure 7.3) connecting the terpyridine groups are observed in the ${ }^{1} \mathrm{H}$ NMR spectra [31,34]. Due to the formation of an octahedral complex, the pyridine rings flip from an antiperiplanar into a synperiplanar conformation. Therefore, the protons in positions 6 and 6" are shifted to higher fields and those in the $3^{\prime}$ and $5^{\prime}$ positions to lower fields by more than $1 \mathrm{ppm}$, the other terpyridine protons showing shifts within $0.3 \mathrm{ppm}$ (Figure 7.4) [35,36]. After each addition, an increase in intensity of the characteristic signals of the complexed polymer units and a concomitant decrease of the corresponding signals of the uncomplexed ones are observed. The presence of distinct sets of signals of the complexed and non-complexed terpyridine moieties is in agreement with the high stability constant reported for the $\mathrm{Fe}^{2+}$ (terpyridine) $)_{2}$ complex in water [37]. When the 1:2 stoichiometric ratio between $\mathrm{Fe}^{2+}$ ions and terpyridine moieties was reached, the signals of the free ligand fully disappeared. 


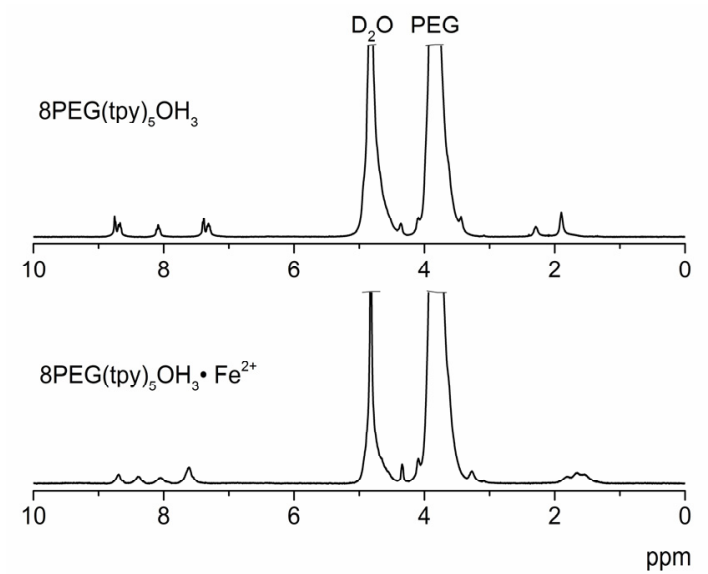

Figure 7.3 ${ }^{1} \mathrm{H}$ NMR spectra of $8 \mathrm{PEG}(\text { tpy })_{5} \mathrm{OH}_{3}$ and $8 \mathrm{PEG}(\text { tpy })_{5} \mathrm{OH}_{3} \cdot \mathrm{Fe}^{2+}\left(\mathrm{D}_{2} \mathrm{O}\right)$.

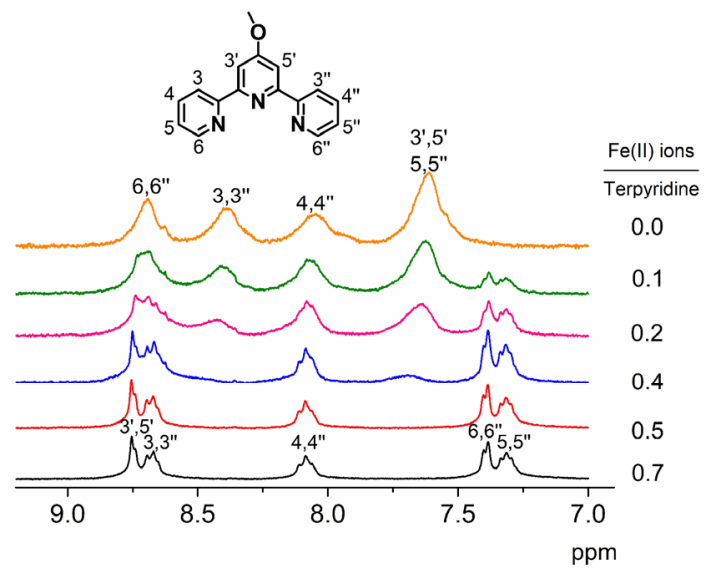

Figure 7.4 Aromatic region of the ${ }^{1} \mathrm{H}$ NMR spectra $\left(\mathrm{D}_{2} \mathrm{O}\right)$ recorded during titration experiments at different $\mathrm{Fe}^{2+}$ :terpyridine molar ratios.

In order to investigate the aggregation behavior of $8 \mathrm{PEG}(\text { tpy })_{5} \mathrm{OH}_{3}$ and its complex (8PEG(tpy) $\left.)_{5} \mathrm{OH}_{3} \cdot \mathrm{Fe}^{2+}\right)$ in water, DLS, ${ }^{1} \mathrm{H}$ NMR and SEM experiments were performed. DLS measurements on $5 \mathrm{mg} \mathrm{mL} \mathrm{mL}^{-1}$ suspensions showed that $8 \mathrm{PEG}(\mathrm{tpy})_{5} \mathrm{OH}_{3}$ forms nanoparticles with an average size distribution of $10 \mathrm{~nm}$ (Figure 7.5 left). Upon titration with a $\mathrm{FeCl}_{2}$ solution the formation of larger nanogels, displaying a monomodal size distribution with an average diameter of $250 \mathrm{~nm}$, was observed. This distribution did not change upon addition of $\mathrm{Fe}^{2+}$ in excess. Nanogels with similar average size were detected in SEM images (Figure 7.5 right). 

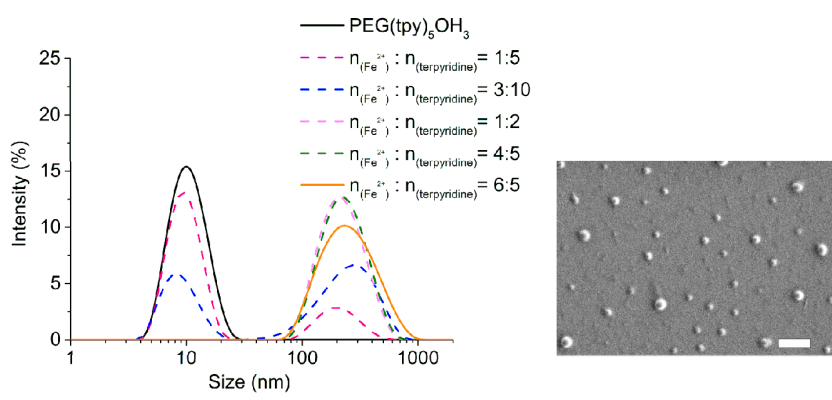

Figure 7.5 Left: DLS intensity plots showing the size distribution profiles of $8 \mathrm{PEG}(\text { tpy })_{5} \mathrm{OH}_{3} \cdot \mathrm{Fe}^{2+}$ complexes at different $\mathrm{Fe}^{2+}$ :terpyridine ratios. The polymer concentration was $5 \mathrm{mg} \mathrm{mL}$. Right: SEM image of $8 \mathrm{PEG}(\text { tpy })_{5} \mathrm{OH}_{3} \cdot \mathrm{Fe}^{2+}$ nanogels in deionized water (bar is $1 \mu \mathrm{m}$ ).

The distinct aggregation behavior apparent from the DLS experiments was also reflected in the differences in signal linewidths in the ${ }^{1} \mathrm{H}$ NMR spectra of $8 \mathrm{PEG}(\operatorname{tpy})_{5} \mathrm{OH}_{3}$ and $8 \mathrm{PEG}(\mathrm{tpy})_{5} \mathrm{OH}_{3} \cdot \mathrm{Fe}^{2+}$ complexes shown (Figure 7.3). In particular, protons in the terpyridine units (in the 7-9 ppm region) and in the 8PEG-tpy linking chains (1-3 ppm) showed broad signals for $8 \mathrm{PEG}(\text { tpy })_{5} \mathrm{OH}_{3}$ but quite narrow ones for $8 \mathrm{PEG}(\mathrm{tpy})_{5} \mathrm{OH}_{3}$. The significantly different linewidths indicate that the hydrophobic terpyridine moieties tend to self-aggregate in water, whereas the positively charged $\mathrm{Fe}^{2+}$ :terpyridine terminal units of the complexed polymer are more soluble in water and hence are highly mobile.

Further insights into the aggregation behavior of the terpyridine units in 8PEG(tpy) $)_{5} \mathrm{OH}_{3}$ came from ${ }^{1} \mathrm{H}$ NMR experiments performed at different concentrations and temperatures. The spectra run on samples with concentration in the 1 to $10 \mathrm{wt} \%$ range did not show any significant difference either in chemical shifts or linewidths (data not shown). On the other hand, significant downfield shifts were observed for the aromatic and linking chain protons upon heating, with only a slight narrowing of linewidths (Figure 7.6). The temperature dependence observed indicates that an equilibrium is present between two limiting situations, possibly identifiable in an aggregated and a loosened structure. The former could arise from terpyridine $\pi-\pi$ stacking and hydrogen bonding between nearby amide groups. Indeed, $\pi-\pi$ stacking of aromatic molecules typically induce upfield shifts of the NMR signals of the aromatic protons [38]. Such shifts are generally concentration and temperature dependent, increasing with increasing concentrations and decreasing with increasing temperatures [39]. 


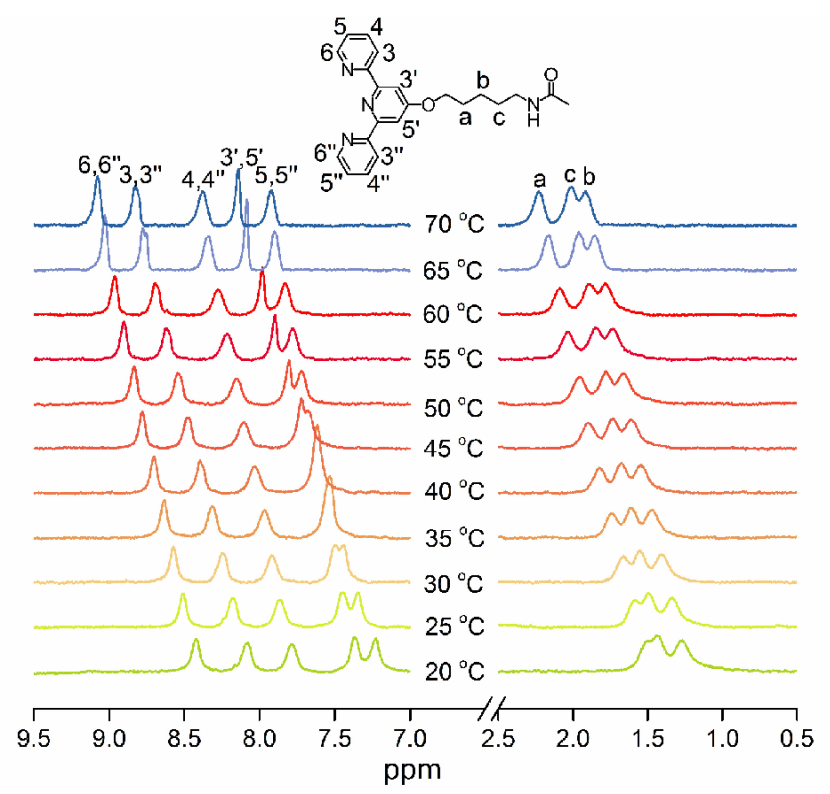

Figure 7.6 Selected regions of ${ }^{1} \mathrm{H}$ NMR spectra recorded on $10 \mathrm{wt} \% 8 \mathrm{PEG}(\operatorname{tpy})_{5} \mathrm{OH}_{3}$ in $\mathrm{D}_{2} \mathrm{O}$ at different temperatures.

As shown in Figure 7.6 the chemical shifts of terpyridine protons moved downfield as the temperature increased and the equilibrium was shifted to a less aggregated structure. Similar temperature dependence was observed for the protons of the linking chains, suggesting the participation of the amide groups to the stabilization of the stacked structure possibly through hydrogen bonding. However, differently from what commonly observed, no concentration dependence was here highlighted. This could be due to the peculiar star architecture of the $8 \mathrm{PEG}(\mathrm{tpy})_{5} \mathrm{OH}_{3}$ molecules which allows the formation of an intramolecular stacked structure surrounded by PEG chains which limits the interaction with terpyridine units belonging to other molecules.

\subsubsection{Mechanical properties and morphology of the hydrogels}

On the basis of ${ }^{1} \mathrm{H}$ NMR, DLS and SEM data, and considering the PEG arm length, estimated on the order of $20 \mathrm{~nm}$ in the all trans configuration, it seems plausible that $8 \mathrm{PEG}(\text { tpy })_{5} \mathrm{OH}_{3}$ self-aggregates in water forming unimolecular micelles. Upon $\mathrm{Fe}^{2+}-$ 
terpyridine complex formation, the terminal terpyridine units become water soluble, so that intermolecular complexation becomes favored, giving rise to larger nanogels at low concentrations and to hydrogels at higher concentrations. In fact, at $8 \mathrm{PEG}(\operatorname{tpy})_{5} \mathrm{OH}_{3}$ polymer concentrations higher than $3 \mathrm{wt} \%$ addition of an $\mathrm{FeCl}_{2}$ solution containing a stoichiometric amount of $\mathrm{Fe}^{2+}$ ions with respect to the terpyridine groups $\left(\mathrm{Fe}^{2+}\right.$ :terpyridine molar ratio of 1:2) afforded hydrogels. Gelation was instantaneous and hydrogels prepared at different concentrations were directly used for mechanical measurements. Oscillatory rheology experiments were applied to determine the storage modulus ( $\left.\mathrm{G}^{\prime}\right)$ and loss modulus (G') of the hydrogels at concentrations ranging from 5 to $20 \mathrm{wt} \%$. The storage modulus of the hydrogels increased with increasing polymer concentration as a result of the increasing cross-linking density (Figure 7.7 left). As an example, at polymer concentrations of $5 \mathrm{wt} \%$ and $8 \mathrm{wt} \%$ the storage modulus was 1.4 and $4.8 \mathrm{kPa}$, respectively. The damping factor ( $\tan \delta=\mathrm{G}^{\prime} / \mathrm{G}^{\prime}$ ) of the hydrogels largely decreased with increasing polymer concentration from $24.7 \times 10^{-3}$ to $4.9 \times 10^{-3}$, indicating that more elastic networks were formed. In addition, strain sweep results showed that all hydrogels at concentrations ranging from 5 to $20 \mathrm{wt} \%$ had a constant storage modulus up to $10 \%$ strain (Figure 7.8 left). Subsequent frequency sweeps from 0.1 to $100 \mathrm{~Hz}$ at $1 \%$ strain showed that the storage moduli exhibited a constant value over the whole range indicative of a stable, cross-linked network (Figure 7.8 right).

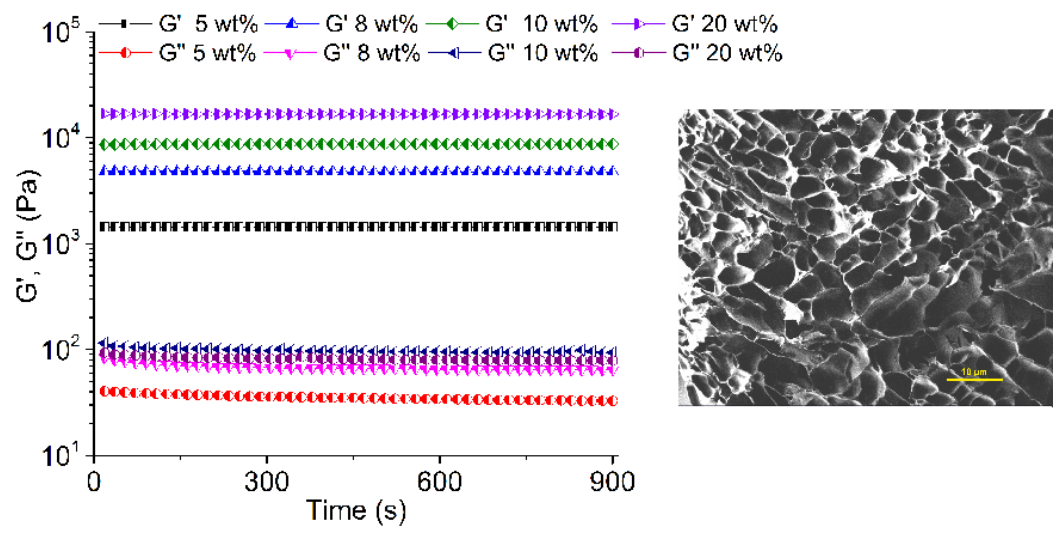

Figure 7.7 Left: Storage moduli (G') and loss moduli (G') of $8 \mathrm{PEG}(\text { tpy })_{5} \mathrm{OH}_{3} \cdot \mathrm{Fe}^{2+}$ complex hydrogels at different polymer concentrations. A frequency of $1 \mathrm{~Hz}$ and a strain of $1 \%$ were applied. Right: SEM image of an $8 \mathrm{wt} \% 8 \mathrm{PEG}(\text { tpy })_{5} \mathrm{OH}_{3} \cdot \mathrm{Fe}^{2+}$ hydrogel. 

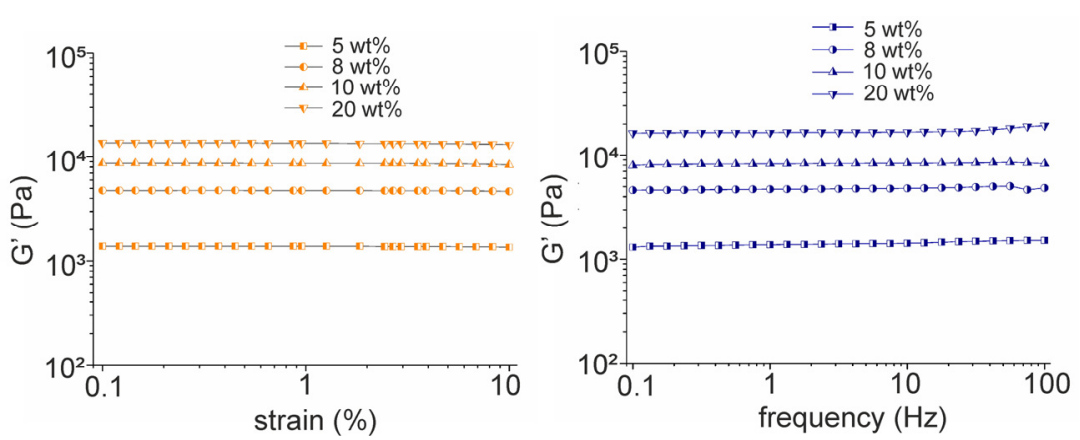

Figure 7.8 Strain (left) and frequency (right) sweep of $\mathrm{Fe}^{2+}$-terpyridine complexed gels at different concentrations.

The morphology of an $8 \mathrm{wt} \% \quad 8 \mathrm{PEG}(\mathrm{tpy})_{5} \mathrm{OH}_{3} \cdot \mathrm{Fe}^{2+}$ freeze-dried hydrogel was determined using scanning electron microscopy (SEM) (Figure 7.7 right) showing a highly porous structure with well-interconnected pores.

\subsubsection{Chondrocytes culture with nanogels or hydrogels}

In view of the potential biomedical and pharmaceutical applications of 8PEG(tpy $)_{5} \mathrm{OH}_{3} \cdot \mathrm{Fe}^{2+}$, cytotoxicity of both $8 \mathrm{PEG}(\text { tpy })_{5} \mathrm{OH}_{3}$ and $8 \mathrm{PEG}(\text { tpy })_{5} \mathrm{OH}_{3} \cdot \mathrm{Fe}^{2+}$ nanogels was investigated through direct contact with chondrocytes. The cytotoxicity of the $8 \mathrm{PEG}(\mathrm{tpy})_{5} \mathrm{OH}_{3} \cdot \mathrm{Fe}^{2+}$ hydrogels was studied by indirect contact with chondrocytes by using a transwells system and finally the biocompatibility of the gels incorporated with chondrocytes was investigated via a live-dead assay.
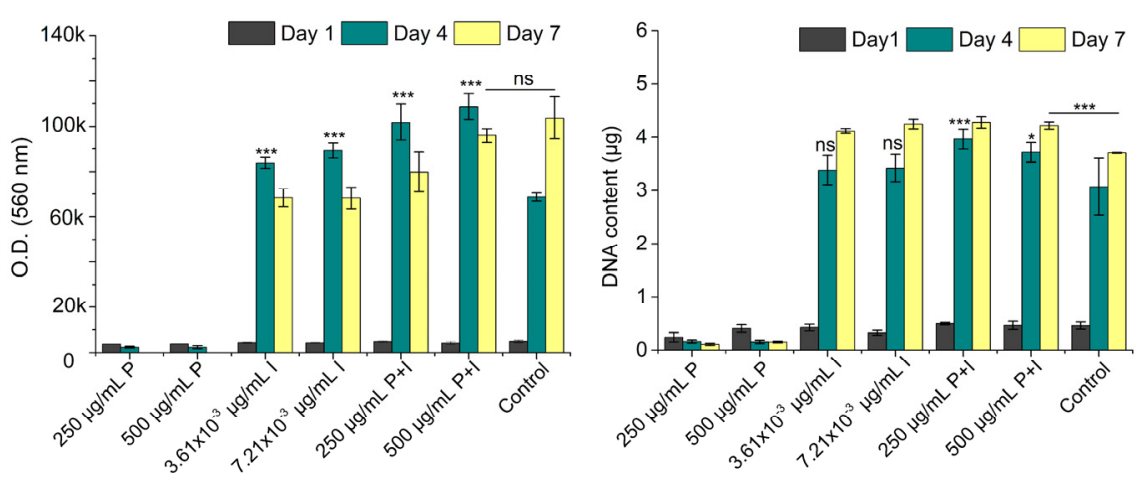

Figure 7.9 Metabolic activity (left) and DNA content (right) of chondrocytes in the 
presence of $8 \mathrm{PEG}(\text { tpy })_{5} \mathrm{OH}_{3}(\mathrm{P}), \mathrm{FeCl}_{2}$ solutions (I) and $8 \mathrm{PEG}(\mathrm{tpy})_{5} \mathrm{OH}_{3} \cdot \mathrm{Fe}^{2+}(\mathrm{P}+\mathrm{I})$ at different concentrations (cell seeding density: 5000 cells/well).

In direct cytotoxicity tests, chondrocytes were exposed to $\mathrm{FeCl}_{2}$ solutions at concentrations of 3.83 or $7.66 \mu \mathrm{g} \mathrm{mL}^{-1}$, and to polymer solution of $8 \mathrm{PEG}(\mathrm{tpy})_{5} \mathrm{OH}_{3}$ at 250 or $500 \mu \mathrm{g} \mathrm{mL} L^{-1}$ and $8 \mathrm{PEG}(\mathrm{tpy})_{5} \mathrm{OH}_{3} \cdot \mathrm{Fe}^{2+}\left(\mathrm{Fe}^{2+}\right.$ :terpyridine molar ratio of $\left.1: 2\right)$ nanogel suspensions at concentrations of 250 and $500 \mu \mathrm{g} \mathrm{mL}^{-1}$ for 1, 4 and 7 days. Cell survival of the chondrocytes at these different conditions was evaluated using PrestoBlue (PB) and CyQUANT DNA assays. The assays revealed no metabolic activity of the chondrocytes and a very low DNA content in the presence of $8 \mathrm{PEG}(\mathrm{tpy})_{5} \mathrm{OH}_{3} \mathrm{compared}$ to the control at all measuring time points (Figure 7.9) indicating that the uncomplexed polymer is cytotoxic. Importantly, cell metabolic activity was higher in the presence of the $8 \mathrm{PEG}(\mathrm{tpy})_{5} \mathrm{OH}_{3} \cdot \mathrm{Fe}^{2+}$ nanogel suspensions or in the presence of $\mathrm{Fe}^{2+}$ ions compared to the control at day 4 but slightly lower at day 7. Similar trends were found for the DNA content.
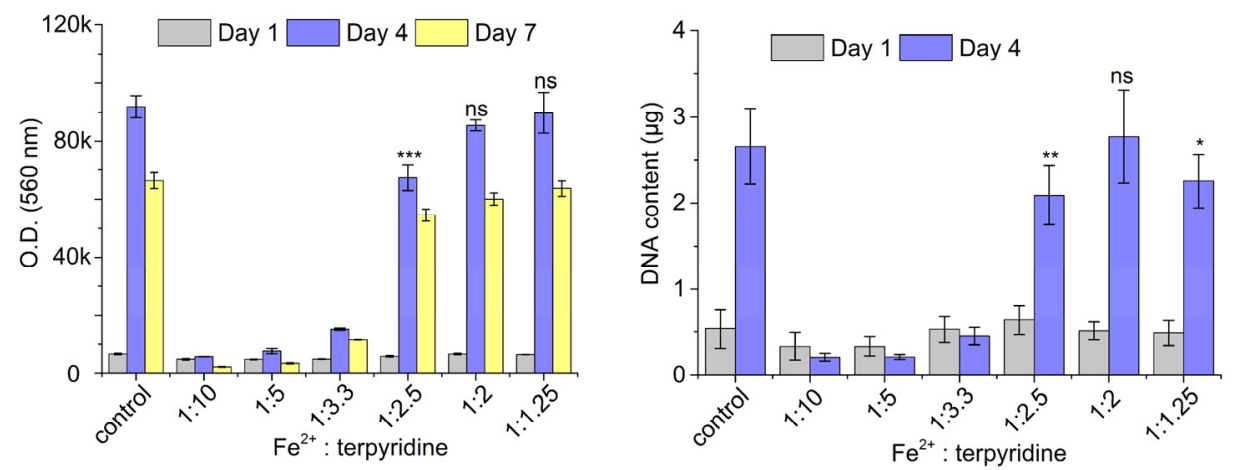

Figure 7.10 Metabolic activity (left) and DNA content (right) of chondrocytes cultured at different complex ratios of $8 \mathrm{PEG}(\mathrm{tpy})_{5} \mathrm{OH}_{3}$ and $\mathrm{Fe}^{2+}$. Cell seeding density: 5000 cells/well.

The cytotoxicity of the uncomplexed polymer was also determined at different $\mathrm{Fe}^{2+}$ ion: terpyridine molar ratios (Figure 7.10). At low ratios the partially complexed nanogels $\left(100 \mu \mathrm{g} \mathrm{mL}^{-1}\right)$ appeared cytotoxic to chondrocytes. Even at a $\mathrm{Fe}^{2+}$ :terpyridine molar ratio of 1:2.5 the metabolic activity and DNA content was significant lower than 
the control. At a $\mathrm{Fe}^{2+}$ :terpyridine molar ratio of 1:2, no significant difference was found both in metabolic activity and DNA content compared to the control. In the presence of an excess of $\mathrm{Fe}^{2+}$ ions, the metabolic activity was not affected whereas a slightly lower DNA content compared to the control was found. The results pointed to cytotoxicity of uncomplexed and partially complexed $8 \mathrm{PEG}(\text { tpy })_{5} \mathrm{OH}_{3}$ possibly due to depletion of essential metal ions by complexation with terpyridine groups.

Considering that cytotoxicity could be induced by leaching of compounds, $8 \mathrm{PEG}(\text { tpy })_{5} \mathrm{OH}_{3} \cdot \mathrm{Fe}^{2+}$ hydrogels were put in transwells and brought in indirect contact with chondrocytes for 1, 4 and 7 days. Chondrocyte proliferation was measured by a PrestoBlue and CyQUANT DNA assay. Compared to the controls (4PEG-DA hydrogels and empty transwells) no significant differences were found over a culturing period of 7 days (Figure 7.11). Because of the very small dissociation rate constant of the $\mathrm{Fe}^{2+}$ bis(terpyridine) complex $\left(8.1 \times 10^{-7} \mathrm{~s}^{-1}\right)$ the concentration of free ligand and mono complexes is very low [25]. Furthermore, the high stability of the $8 \mathrm{PEG}(\text { tpy })_{5} \mathrm{OH}_{3} \cdot \mathrm{Fe}^{2+}$ with respect to other metal ions, reduces the possibility of essential metal ions depletion.
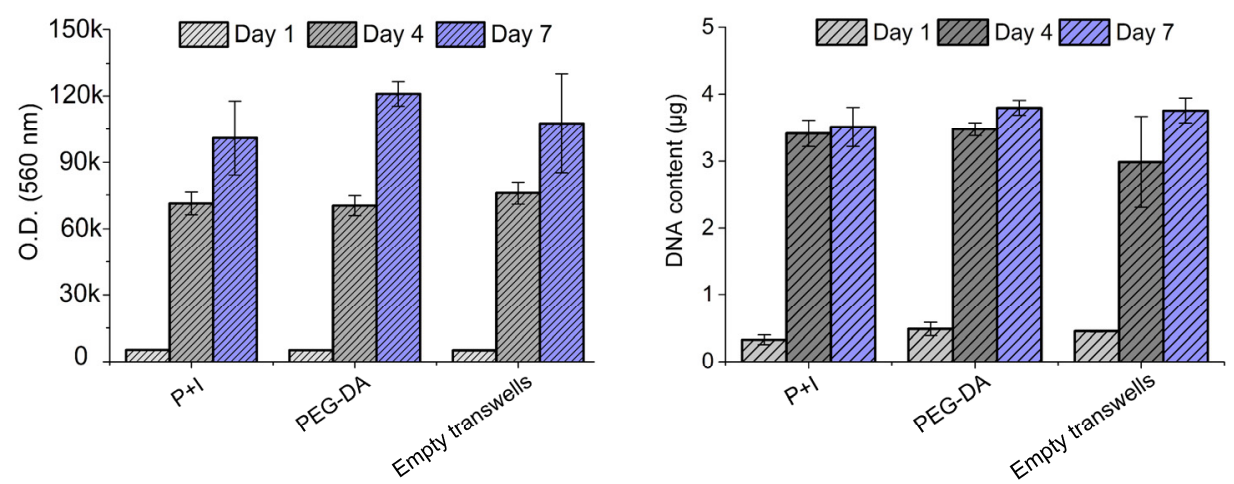

Figure 7.11 Metabolic activity (left) and DNA content (right) of chondrocytes in indirect contact with $8 \mathrm{PEG}(\text { tpy })_{5} \mathrm{OH}_{3} \cdot \mathrm{Fe}^{2+}$ hydrogels $(\mathrm{P}+\mathrm{I})$, 4PEG-DA hydrogels. Cell seeding density: 5000 cells/well.

The cytocompatibility of the $8 \mathrm{PEG}(\text { tpy })_{5} \mathrm{OH}_{3} \cdot \mathrm{Fe}^{2+}$ hydrogels at different concentrations was investigated by the incorporation of bovine chondrocytes into the gels and determining cell survival by a live-dead assay (Figure 7.12). In all cases, over $90 \%$ of the chondrocytes stained green, indicating cytocompatibility of the complexes. 
Furthermore, fluorescence microscopy, as presented in Figure 9, revealed slightly more dead cells present in the $10 \mathrm{wt} \%$ hydrogels compared to hydrogels prepared at lower polymer concentrations, indicating that the crosslink density affects cell viability. Therefore, when comparing both the mechanical properties and biological properties of the hydrogels, the $8 \mathrm{wt} \%$ hydrogels provided better conditions for cell growth. The microscope pictures also revealed a lower cell density in the hydrogels than in the agarose controls. Importantly, cells encapsulated in the hydrogels migrated to the surface and detached from the hydrogels in time, indicating the lack of anchoring points for the cells to the hydrophilic polymer chains.

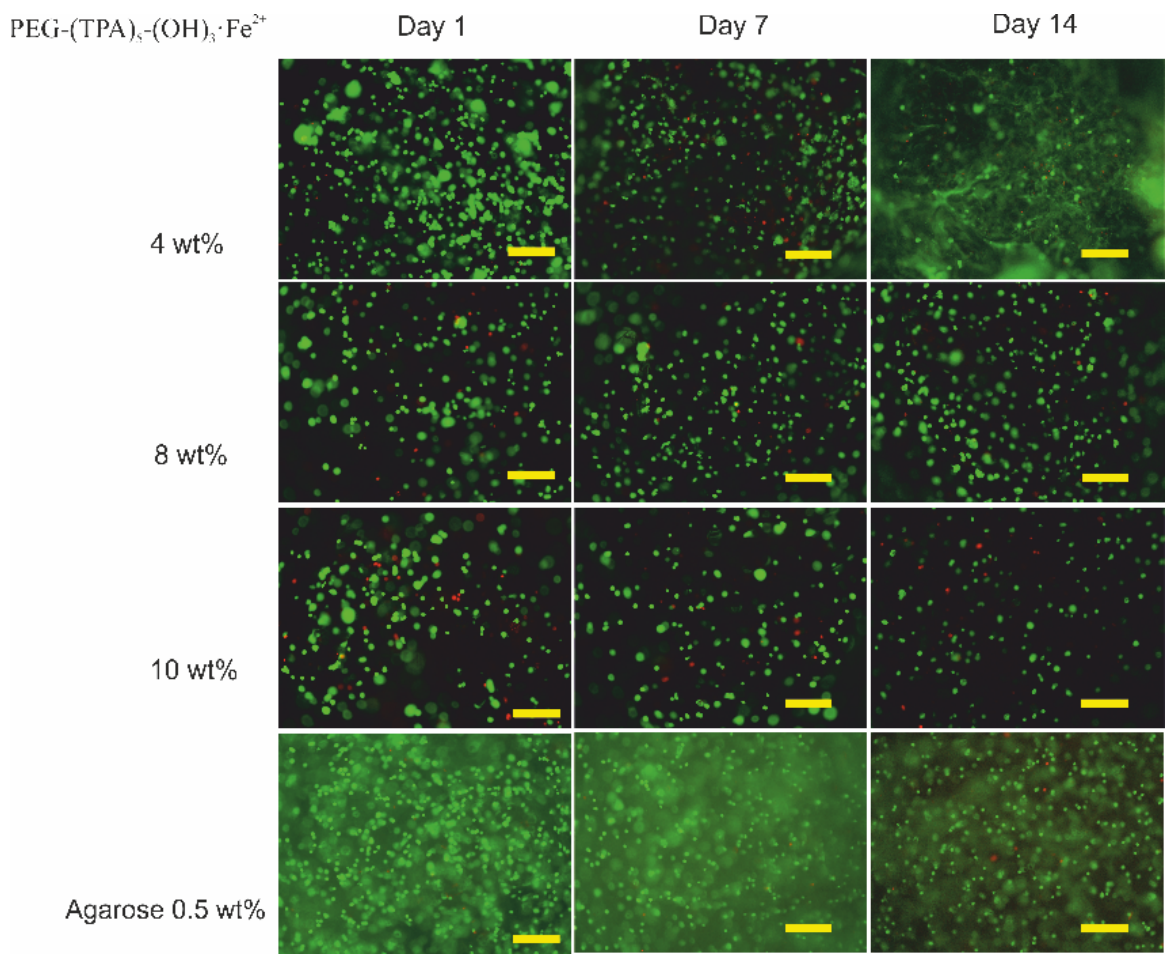

Figure 7.12 Live-dead assay showing chondrocytes incorporated in 4, 8 and $10 \mathrm{wt} \%$ $8 \mathrm{PEG}(\text { tpy })_{5} \mathrm{OH}_{3} \cdot \mathrm{Fe}^{2+}$ hydrogels after 1,7 and 14 days in culture. An agarose gel $(0.5$ wt $\%$ ) was used as control. Scale bar: $200 \mu \mathrm{m}$. 


\subsection{Conclusions}

In-situ supramolecular cross-linking of a terpyridine end group functionalized 8 arm poly(ethylene glycol) with $\mathrm{Fe}^{2+}$ ions afforded nanogels with an average diameter of 250 $\mathrm{nm}$ or hydrogels depending on the polymer concentration. Direct and indirect cytotoxicity tests using bovine chondrocytes revealed the polymer conjugate in the complexed form nontoxic to cells. In the uncomplexed form the polymer conjugate was toxic to the cells likely due to depletion of trace metal ions by complexation. Studies on hydrogels placed in transwells showed that no leaching of toxic compounds takes place. Chondrocytes incorporated in the hydrogels maintained high cell viability. This study indicates that the high kinetic stability of $\mathrm{Fe}^{2+}$ complexes of polymer conjugated terpyridine groups provides a start for the use of supramolecular complexes in potential biomedical or pharmaceutical applications.

\section{References}

[1] Peppas, N. A.; Bures, P.; Leobandung, W.; Ichikawa, H., Hydrogels in pharmaceutical formulations. Eur. J. Pharm. Biopharm. 2000, 50 (1), 27-46.

[2] Hennink, W. E.; van Nostrum, C. F., Novel crosslinking methods to design hydrogels. Adv. Drug Deliv. Rev. 2002, 54 (1), 13-36.

[3] Jeong, B.; Kim, S. W.; Bae, Y. H., Thermosensitive sol-gel reversible hydrogels. Adv. Drug Deliv. Rev. 2002, 54 (1), 37-51.

[4] Berger, J.; Reist, M.; Mayer, J. M.; Felt, O.; Peppas, N. A.; Gurny, R., Structure and interactions in covalently and ionically crosslinked chitosan hydrogels for biomedical applications. Eur. J. Pharm. Biopharm. 2004, 57 (1), 19-34.

[5] Kopecek, J.; Yang, J. Y., Revie., Hydrogels as smart biomaterials. Polym. Int. 2007, 56 (9), 1078-1098.

[6] Ahn, S. K.; Kasi, R. M.; Kim, S. C.; Sharma, N.; Zhou, Y. X., Stimuli-responsive polymer gels. Soft Matter 2008, 4 (6), 1151-1157. 
[7] Tomme, S. R.; Storm, G.; Hennink, W. E., In situ gelling hydrogels for pharmaceutical and biomedical applications. Int. J. Pharm. 2008, 355 (1-2), 1-18.

[8] Muzzarelli, R. A. A., Genipin-crosslinked chitosan hydrogels as biomedical and pharmaceutical aids. Carbohydr. Polym. 2009, 77 (1), 1-9.

[9] Muzzarelli, R. A. A., Chitins and chitosans for the repair of wounded skin, nerve, cartilage and bone. Carbohydr. Polym. 2009, 76 (2), 167-182.

[10] Maarten van Dijk, M.; Rijkers, D. T. S.; Liskamp, R. M. J.; van Nostrum, C. F.; Hennink, W. E., Synthesis and Applications of Biomedical and Pharmaceutical Polymers via Click Chemistry Methodologies. Bioconjugate Chem. 2009, 20 (11), 2001-2016.

[11] Steed, J. W., Atwood, J.L., Nature of supramolecular interactions. In Supramolecular Chemistry, , 2 ed.; John Wiley \& Sons, Ltd.: 2009; pp 27-36.

[12] Hoffman, A. S., Hydrogels for biomedical applications. Adv. Drug Deliv. Rev. 2002, 54 (1), 3-12.

[13] Albrecht, M., Supramolecular chemistry - general principles and selected examples from anion recognition and metallosupramolecular chemistry. Naturwissenschaften 2007, 94 (12), 951-966.

[14] König, B. Vorlesung Supramolekulare Chemie; Regensburg, 2009; pp pp.1-72.

[15] Weng, W. G.; Beck, J. B.; Jamieson, A. M.; Rowan, S. J., Understanding the mechanism of gelation and stimuli-responsive nature of a class of metallosupramolecular gels. J. Am. Chem. Soc. 2006, 128 (35), 11663-11672.

[16] Brassinne, J.; Fustin, C. A.; Gohy, J. F., Polymer Gels Constructed Through MetalLigand Coordination. J. Inorg. Organomet. Polym. 2013, 23 (1), 24-40.

[17] Chiper, M., Hoeppener, S., Schubert, U.S., Fustin, C.-A., Gohy, J.-F., Self-Assembly Behavior of Bis(terpyridine) and Metallo-bis(terpyridine) Pluronics in Dilute Aqueous Solutions. Macromol. Chem. Phys. 2010, 211 (21), 2323-2330.

[18] Jochum, F. D., Brassine, J., Fustin, C.-A., Gohy, J.-F., Metallo-supramolecular hydrogels based on copolymers bearing terpyridine side-chain ligands. Soft Matter 2013, 9 (7), 2314-2320.

[19] Kimura, M.; Nakagawa, Y.; Adachi, N.; Tatewaki, Y.; Fukawa, T.; Shirai, H., Metallosupramolecular Gels Made of Four-armed Poly(ethylene glycol)s Having Terpyridine Termini. Chem. Lett. 2009, 38 (4), 382-383. 
[20] Asoh, T. A.; Yoshitake, H.; Takano, Y.; Kikuchi, A., Fabrication of Self-Healable Hydrogels through Sol-Gel Transition in Metallo-supramolecular Aqueous Solution by Aeration. Macromol. Chem. Phys. 2013, 214 (22), 2534-2539.

[21] Rossow, T.; Bayer, S.; Albrecht, R.; Tzschucke, C. C.; Seiffert, S., Supramolecular Hydrogel Capsules Based on PEG: A Step Toward Degradable Biomaterials with Rational Design. Macromol. Rapid Commun. 2013, 34 (17), 1401-1407.

[22] Rossow, T.; Seiffert, S., Supramolecular polymer gels with potential model-network structure. Polym. Chem. 2014, 5 (8), 3018-3029.

[23] Rossow, T.; Habicht, A.; Seiffert, S., Relaxation and Dynamics in Transient Polymer Model Networks. Macromolecules 2014, 47 (18), 6473-6482.

[24]Ueki, T.; Takasaki, Y.; Bundo, K.; Ueno, T.; Sakai, T.; Akagi, Y.; Yoshida, R., Autonomous viscosity oscillation via metallo-supramolecular terpyridine chemistry of branched poly(ethylene glycol) driven by the Belousov-Zhabotinsky reaction. Soft Matter 2014, 10 (9), 1349-1355.

[25] Hackelbusch, S.; Rossow, T.; Becker, H.; Seiffert, S., Multiresponsive Polymer Hydrogels by Orthogonal Supramolecular Chain Cross-Linking. Macromolecules 2014, 47 (12), 4028-4036.

[26] Wang, R.; Geven, M.; Dijkstra, P. J.; Martens, P.; Karperien, M., Hydrogels by supramolecular crosslinking of terpyridine end group functionalized 8 arm poly(ethylene glycol). Soft Matter 2014, 10 (37), 7328-7336.

[27] Jin, R.; Moreira Teixeira, L. S.; Dijkstra, P. J.; Karperien, M.; van Blitterswijk, C. A.; Zhong, Z. Y.; Feijen, J., Injectable chitosan-based hydrogels for cartilage tissue engineering. Biomaterials 2009, 30 (13), 2544-2551.

[28] Boncler, M.; Rozalski, M.; Krajewska, U.; Podsedek, A.; Watala, C., Comparison of PrestoBlue and MTT assays of cellular viability in the assessment of antiproliferative effects of plant extracts on human endothelial cells. J. Pharmacol. Toxicol. Methods 2014, 69 (1), 9-16.

[29] Hofmeier, H.; El-ghayoury, A.; Schenning, A.; Schubert, U. S., New supramolecular polymers containing both terpyridine metal complexes and quadruple hydrogen bonding units. Chem. Commun. 2004, (3), 318-319.

[30] Beck, J. B.; Ineman, J. M.; Rowan, S. J., Metal/ligand-induced formation of metallosupramolecular polymers. Macromolecules 2005, 38 (12), 5060-5068. 
[31] Schmatloch, S.; Gonzalez, M. F.; Schubert, U. S., Metallo-supramolecular diethylene glycol: Water-soluble reversible polymers. Macromol. Rapid Commun. 2002, 23 (16), 957-961.

[32] Lohmeijer, B. G. G.; Schubert, U. S., Water-soluble building blocks for terpyridinecontaining supramolecular polymers: Synthesis, complexation, and $\mathrm{pH}$ stability studies of poly(ethylene oxide) moieties. Macromol. Chem. Phys. 2003, 204 (8), 1072-1078.

[33] Schmatloch, S.; van den Berg, A. M. J.; Alexeev, A. S.; Hofmeier, H.; Schubert, U. S., Soluble high-molecular-mass poly(ethylene oxide)s via self-organization. Macromolecules 2003, 36 (26), 9943-9949.

[34] Schmatloch, S.; Schubert, U. S., Engineering with metallo-supramolecular polymers: Linear coordination polymers and networks. Macromol. Symp. 2003, 199, 483-497.

[35] Elsbernd, H.; Beattie, J. K., NMR-spectra of terpyridine and bis-terpyridine complexes of cobalt(III) and iron(II). J. Inorg. Nucl. Chem. 1972, 34 (2), 771-\&.

[36] Pazderski, L.; Pawlak, T.; Sitkowski, J.; Kozerski, L.; Szlyk, E., H-1, C-13, N-15 NMR coordination shifts in Fe(II), Ru(II) and Os(II) cationic complexes with 2,2 :6 ',2 "-terpyridine. Magn. Reson. Chem. 2011, 49 (5), 237-241.

[37]Holyer, R. H.; Hubbard, C. D.; Kettle, S. F. A.; Wilkins, R. G., Kinetics of replacement reactions of complexes of transition metals with 2,2',2"-terpyridine. Inorg. Chem. 1966, 5 (4), 622-\&.

[38] Mitchell, P. R.; Sigel, H.,Pproton nmr-study of self-stacking in purine and pyrimidine nucleosides and nucleotides. Eur. J. Biochem. 1978, 88 (1), 149-154.

[39] Arena, G.; Calogero, G.; Campagna, S.; Scolaro, L. M.; Ricevuto, V.; Romeo, R., Synthesis, characterization, absorption spectra, and luminescence properties of organometallic platinum(II) terpyridine complexes. Inorg. Chem. 1998, 37 (11), 2763-2769. 


\section{Summary}

In-situ forming hydrogels are excellent candidates to be used as scaffolds in biomedical and pharmaceutical applications, like tissue regeneration and drug delivery systems. These systems are also called injectable hydrogels because of their initial flowing nature and hydrogel formation at the location site upon injection. These systems allow minimally invasive surgical procedures, easy incorporation of bioactive molecules or cells and a good fit in a cavity or a defect. These advantages address attractive strategies in the development of in-situ forming hydrogels for specific tissue engineering applications. In addition, the design of such materials is directed to biodegradable polymeric materials with a limited time span, of which degradation is in pace with new tissue formation.

From a material's point of view the design of a biodegradable injectable hydrogel is based on hydrophilic natural or synthetic polymers. These materials may be chemically modified or combined with hydrophobic polymers, a basis for the preparation of either chemically or physically crosslinked networks. Combinations of these have been the basis for hybrid and dually crosslinked networks. In the past decades a wide variety of polymeric hydrogels have been described in literature, covering a wide variety of chemical physical and mechanical properties. In the foregoing years within the research group Developmental BioEngineering new steps were taken in the macromolecular engineering of injectable hydrogels by enzymatic crosslinking with emphasis on the translation of these materials into clinically relevant products. Parallel to this our research has been directed to new ways that can be applied in the macromolecular engineering of in-situ forming hydrogels applying metallo-supramolecular complexation. Transition metal ion complexation by suitable ligands was studied as a strategy to create a single type of crosslinked hydrogels covering the wide range of physical and mechanical properties generally found for crosslinked hydrogels.

In Chapter 1, a general introduction and historical perspective on the developmental bioengineering of articular cartilage regeneration by injectable hydrogels is given. Initial studies on horse cadavers demonstrated that promising results were achieved stimulating further product development ultimately resulting in clinical studies in the near future. In 
Chapter 2, a literature review on the natural polymer dextran and the synthetic polymer star shaped poly(ethylene glycol), is presented as a basis for the research described in this thesis. These polymers are playing an important role in designing injectable hydrogels due their varying chemical, physical and mechanical properties.

In previous studies, enzymatically crosslinked dextran-tyramine (Dex-TA) and hyaluronic acid-tyramine (HA-TA) hydrogels in the presence of horseradish peroxidase (HRP) and hydrogen oxide $\left(\mathrm{H}_{2} \mathrm{O}_{2}\right)$ have proven potent candidates for cartilage regeneration. However, first horse cadaver studies revealed that an inappropriate integration of the injectable hydrogel constructs with the host tissue can easily give rise to failure of the injected construct. In Chapter 3 we demonstrate a strategy for quantitation the adhesion strength between explant cartilage and injectable hydrogels using a home-made tensile test machine. These experiments provided a new strategy to increase the adhesion of the in-situ forming hydrogel by prior priming the host tissue with a polymer precursor. Although the adhesion was largely improved, the fate of the developed injectable hydrogels in the body is yet mysterious. In an initial study we approached the in vivo degradation using real-time imaging and monitoring by conjugation the polysaccharides based injectable hydrogel system with a near-infrared fluorescent (NIR) label (Chapter 4). This system allows the monitoring of the degradation of the hydrogels over extensive long period without the need to sacrifice large number of animals. The degradation profiles showed a slow steady decrease of both on Dex-TA and Dex-TA/HA-TA (50/50) gels, results different from previous in vitro degradation studies. This study indicates the complexity of in vivo enzymatic degradation mechanisms and that prediction of degradation based on in vitro results cannot always be translated to in vivo.

Hyaluronic acid, a component of many tissues, is known to provide viscoelastic properties to tissues. Hydrogels prepared from end-group modified crosslinkable poly(ethylene glycols), are generally elastic. Combining the advantageous properties of these materials was used in the design and development of a new type of injectable applying enzymatic crosslinking by $\mathrm{HRP}$ and $\mathrm{H}_{2} \mathrm{O}_{2}$. In Chapter 5, a novel synthetic polymer, a star shaped 8 arm poly(ethylene glycol)-tyramine (PEG-TA) conjugate was synthesized and enzymatically co-crosslinked with hyaluronic acid-tyramine. Biodegradable highly elastic injectable hydrogels were obtained. Changing the polymer 
ratio provided adequate tuning of the mechanical properties and degradation rates of the injectable hydrogels. These biomimetic hydrogels provide excellent biocompatibility and a suitable network for cells matrix deposition.

A new type of physically crosslinked hydrogels based on an 8 arm poly(ethylene glycol) were explored as injectable hydrogels for biomedical applications (Chapter 6 \& 7). Hydrogels were formed by supramolecular cross-linking using the transition metal ions: $\mathrm{Ni}^{2+}, \mathrm{Fe}^{2+}, \mathrm{Co}^{2+}$ and $\mathrm{Zn}^{2+}$ as guests and terpyridine ligands as hosts. The bond strengths in metal ligand complexes can be as high as those of covalent bonds but also as low as those of electrostatic interactions depending mainly on the relatively thermodynamic and kinetic stability of the host-guest complexes. These supramolecular crosslinked complexes with transition metal ions form nanogels at low concentrations and hydrogels at concentrations above $5 \mathrm{wt} \%$ (Chapter 6). Specifically, the physical and biological properties of the $\mathrm{Fe}^{2+}$-terpyridine-end functionalized 8 arm poly(ethylene glycol) complex were evaluated and described in Chapter 7. Direct and indirect cytotoxicity tests using bovine chondrocytes revealed the polymer conjugate in the complexed form nontoxic to cells. This study indicates that the high kinetic stability of complexes of polymer conjugated terpyridine groups with $\mathrm{Fe}^{2+}$ provides a start for the use of supramolecular complexes in potential biomedical or pharmaceutical applications. 


\section{Samenvatting}

Hydrofiele netwerken die in situ worden gevormd staan sterk in de belangstelling voor biomedische en farmaceutische toepassingen zoals weefselregeneratie en afgifte systemen voor medicijnen. Deze systemen zijn initieel vloeibaar en vormen een netwerk door fysische interactie of chemische crosslinking op de plaats van de injectie en worden daarom ook wel injecteerbare hydrogelen genoemd. Het grote voordeel van deze methode is de minimaal invasieve procedure en het gemakkelijk inmengen van bioactieve stoffen of cellen in de initieel vloeibare systemen. Dit houdt ook in dat bij opvulling van b.v. een defect er een zeer goede aansluiting is van de hydrogel met het omringende weefsel. Omdat het netwerk een tijdelijke functie heeft is in het ontwerpen van dergelijke systemen biodegradatie, afgestemd op de vorming van nieuw weefsel, belangrijk.

Materiaalkundig gezien kunnen we in het ontwerpen van een injecteerbare hydrogel uitgaan van hydrofiele natuurlijke of synthetische polymeren. Deze materialen zijn onder andere chemisch te modificeren en daarbij een basis voor chemisch gecrosslinkte netwerken of te combineren met hydrofobe polymeren voor het maken van fysisch gecrosslinkte netwerken. Ook combinaties van deze methoden zijn mogelijk. In de afgelopen jaren is een grote variëteit aan hydrogelen met specifieke chemische, fysische en mechanische eigenschappen in de literatuur beschreven. In de afgelopen jaren zijn in de onderzoeksgroep "Developmental Bioengineering" nieuwe stappen gezet in de macromoleculaire technologie van injecteerbare hydrogelen door het enzymatisch crosslinken van polymeren, waarbij de nadruk lag op het ontwikkelen van een klinische toepasbaar product. Parallel aan dit onderzoek is een nieuwe technologie onderzocht toepasbaar in het veld van in-situ vormende hydrogelen. In het bijzonder zijn injecteerbare systemen ontwikkeld waarbij de knooppunten in het netwerk worden gevormd door complexen van overgangsmetaalionen met liganden. De strategie was er op gericht verschillende metaalionen te gebruiken in combinatie met een enkel polymeer, zodat de thermodynamische en kinetische stabiliteit van het complex bepalend zou zijn voor de fysische en mechanische eigenschappen van de hydrogel.

In Hoofdstuk 1 is, in een algemene inleiding, het onderzoek naar de regeneratie van kraakbeen middels injecteerbare hydrogelen in een kort historische perspectief gezet. De 
eerste studies op paardenkadavers, waarbij kunstmatig gemaakte kraakbeendefecten werden opgevuld met enzymatisch crosslinkbare hydrofiele polymeren, leverden uitstekende resultaten op en waren een stimulans voor verder onderzoek naar de klinische vertaling van de methode. In Hoofdstuk 2 is een literatuuronderzoek beschreven naar de eigenschappen en chemische modificatie van het natuurlijke hydrofiele polymeren dextran en het synthetische stervormig poly(ethyleen glycol), materialen die een basis waren voor het onderzoek beschreven in dit proefschrift. In het ontwerp van injecteerbare hydrogelen hebben deze materialen een belangrijke rol gespeeld vanwege hun verschillende fysische eigenschappen.

In eerdere studies is aangetoond dat dextran-tyramine (Dex-TA) en hyaluronzuurtyramine (HA-TA) conjugaten hydrogelen vormen door enzymatische crosslinking in de aanwezigheid van het enzym mierikswortel peroxidase in aanwezigheid van waterstofperoxide. De eerste paardenkadaver studies lieten echter ook zien dat verbetering van de hechting van de gel an het omringende weefsel noodzakelijk was. Bij onvoldoende integratie van de gel met het weefsel kon de hydrogel gemakkelijk worden verwijderd door mechanische belasting. Deze oorzaak is de basis geweest voor onderzoek naar een verbetering van de hechting van de gel aan het weefsel en is beschreven in Hoofdstuk 3. De adhesiesterkte tussen geëxplanteerd kraakbeen en hydrogel was te meten door trek rek testen gebruik makend van een aan het Radboud Medisch Centrum in Nijmegen gemaakte mini-trekbank (Prof. Verdonschot). Deze experimenten leverden een nieuwe strategie op om de adhesie tussen de in-situ vormende hydrogel en kraakbeenweefsel groter te maken dan de gelsterkte. De hydrogel heeft slechts een tijdelijke functie en de regeneratie van het weefsel is bij voorkeur afgestemd op de degradatie van de hydrogel. In een eerste studie hebben we de degradatie van geïmplanteerde hydrogelen gelabeld met een nabij-infrarood fluorescent (NIR) label bestudeerd middels ex-vivo imaging en monitoring (Chapter 4). Deze methode heeft als groot voordeel dat slechts enkele proefdieren voor de meting noodzakelijk zijn omdat de verandering van het fluorescente signaal een maat is voor de degradatie. Door de fluorescentie met een camera ex-vivo te meten kon over lange tijd metingen gedaan worden aan geïmplanteerde hydrogelen in een klein aantal proefdieren. De degradatie profielen lieten zien dat een langzame degradatie plaatsvindt en dat deze methode nieuwe inzichten geven in de degradatie van de enzymatisch gecrosslinkte hydrogelen. 
Hyaluronzuur is een belangrijke component in vele weefsels en levert daaraan een belangrijke bijdrage aan de viscoelasticiteit. Anderzijds zijn hydrogelen gemaakt van poly(ethyleen glycol) over het algemeen elastisch. In het ontwerp van enzymatisch gecrosslinkte injecteerbare hydrogelen zijn deze eigenschappen gecombineerd en beschreven in Hoofdstuk 5. Een nieuw synthetisch stervormig 8 arm poly(ethyleen glycol)-tyramine (PEG-TA) conjugaat werd gesynthetiseerd en enzymatisch gecrosslinkt met enn hyaluronzuur-tyramine conjugaat. Elastische hydrogelen met mechanische eigenschappen en degradatiesnelheden werden verkregen afhankelijk van de verhouding tussen de twee polymeer conjugaten. Deze biomimetische hydrogelen hebben een uitstekende biocompatibiliteit en matrix voor het afzetten van een nieuwe kraakbeen extra cellulaire matrix.

Een nieuw type fysisch gecrosslinkte hydrogelen gebaseerd op een 8 arm poly(ethyleen glycol) werden onderzocht als basis voor injecteerbare hydrogelen voor biomedische toepassingen (Hoofdstukken 6 \& 7). Door supramoleculaire crosslinking van terpyridine liganden geconjugeerd aan het poly(ethyleen glycol) met overgangsmetaalionen zoals $\mathrm{Ni}^{2+}, \mathrm{Fe}^{2+}, \mathrm{Co}^{2+}$ en $\mathrm{Zn}^{2+}$ werden hydrogelen gemaakt. De bindingsterkten in deze metaal ligand complexen kunnen die van covalente bindingen benaderen maar kunnen ook in de grootte orde liggen van elektrostatische interacties en is afhankelijk van de relatieve thermodynamische en kinetische stabiliteit van het gebruikte metaalion in deze gastheer-gast complexen. Deze supramoleculaire gecrosslinkte complexen vormen nanogelen bij lage concentraties en hydrogelen bij concentraties boven 5 gewichtsprocent en zijn kinetisch stabiel in het geval van $\mathrm{Ni}^{2+}$ en $\mathrm{Fe}^{2+}$ complexen (Hoofdstuk 6). De fysische en biologische eigenschappen van de $\mathrm{Fe}^{2+}-$ terpyridine gefunctionaliseerde 8 armige poly(ethyleen glycol) complexen werden geëvalueerd en beschreven in Hoofdstuk 7. Directe en indirecte cytotoxiciteit testen met kraakbeencellen lieten zien dat complexen niet toxisch zijn voor de cellen. Deze studie laat zien dat kinetisch stabiele complexen van polymeren geconjugeerd met terpyridine liganden en $\mathrm{Fe}^{2+}$ ionen een start kan zijn voor het gebruik van supramoleculaire complexering in biomedische en/of farmaceutische toepassingen. 


\section{摘}

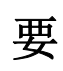

原位形成水凝胶作为优异的组织工程新型材料之一在近些年被广泛用于生物医学工程等方 面的应用, 比如用作组织再生及药物释放的载体等等。由于其初始材料的流动性及注射后 即成型的特点, 这类体系还可称为可注射水凝胶。介于其手术创伤面小, 且亲水的生物活 性物质或细胞易于在其中分散, 而且可修复具有不规则形状的缺损部位, 该体系目前已被 大量应用于特定部位的组织工程修复。此外, 此类材料的设计需要可生物降解的高分子材 料, 以达到在限定的时间内降解, 以及与新组织的形成相辅相成的作用。从材料的角度出 发, 生物可降解型可注射水凝胶的设计是基于亲水性天然高分子或合成聚合物。这些亲水 性与疏水性聚合物可以通过化学修饰或分子间作用结合, 从而制备出或化学交联或物理交 联或以这两种为基础的混合交联或多种交联方式的三维结构。在过去几十年里, 大量文献 描述了聚合物水凝胶, 及其多样化的物理、化学和机械性能。近年来, 我们课题组 (DBE) 研究了利用酶交联的方法制备可注射水凝胶, 重点研究和评价了该材料面向临床相关产品 的发展前景。与此同时, 我们还援引了超分子化学中金属络合的方法实现了制备原位形成 水凝胶, 利用多种过渡金属与三联吡啶络合能力的不同, 设计并评价了通过单一类型的交 联方法得到一系列物理和机械性能各异的可注射水凝胶。

该论文第一章节扼要地介绍了 DBE 课题组利用原位可注射水凝胶对关节软骨修复的研究历 史和现状。这些基础研究及针对大型动物 (马关节) 的临床研究揭示了可注射水凝胶作为 理想修复材料的需求。第二章节针对三种应用广泛且与该论文息息相关的天然或合成材料, 葡聚糖（Dextran）和聚乙二醇（poly(ethylene glycol)）在生物医学及制药学方面的应用进 行了总结和回顾。

在先前的研究中, 辣根过氧化物酶在过氧化氢的催化下交联葡聚糖 dextran-tyramine (DexTA) 和透明质酸 Hyaluronic acid-tyramine (HA-TA) 制备的水凝胶已被证明可用作软骨修复的 候选材料之一。第一章节马关节实验中水凝胶在生理盐水冲洗下出现脱落现象, 印证了无 黏附能力的可注射水凝胶根本无法与宿主组织产生整合也同时失去了暂时取代组织的意义。 基于此, 在第三章节中, 我们利用自主设计的拉伸测试仪对外植体软骨和水凝胶进行拉伸 测试, 这种新的实验技术增强了我们对于材料与宿主组织黏附的认识, 这一实验结果对于 可注射水凝胶的临床应用提供了很高的使用价值。由于生物材料的降解特性, 尽管水凝胶 具有较强的组织黏附能力, 但是随着时间更迭其在体内的命运仍然不得而知, 在第四章节 中我们进一步研究了可以在体外实时成像和观测的近红外荧光 (NIR) 标记可注射水凝胶。 
该体系允许我们监测水凝胶材料的体内降解过程, 其具有时间广度长, 使用动物量少, 且 易于操作等优点。裸鼠体内降解实验表明 Dex-TA 和 Dex-TA / HA-TA (50/50) 凝胶都具有 稳定缓慢的降解过程, 这与之前课题组得到的体外实验结果有异, 也侧面证实了体内酶降 解机制的复杂性和难预测性。

透明质酸 (HA) 是众多组织的一个重要组成部分, 其主要作用为为组织提供粘弹性能, 而末端改性的聚乙二醇交联后一般具有弹性。在第五章节中, 我们结合这两种材料的优越 性能研发了一种新型的具有高弹性的可注射水凝胶。改变聚合物混合比例可适当调节其力 学性能和降解速率。这类仿生水凝胶具有优良的生物相容性以及可供细胞基质附着的网络 结构。

论文在第六、七章节中介绍了一种新型的物理交联水凝胶, 我们利用过渡金属与三联吡啶 修饰的聚乙二醇进行超分子化学中的配位交联制备了具有多种物理性能和机械性能的原位 形成水凝胶。其基础配位能力, 热稳定性和动力学性能详述于第六章节。我们对二价铁离 子形成的配位凝胶进行了生物医学方面的研究, 通过直接和间接使用牛软骨细胞进行细胞 毒性测试, 揭示了聚合物与金属配位后对细胞无毒害作用, 评价结果详述于第七章节。本 研究表明化学改性聚乙二醇与二价铁离子配位形成的凝胶具有较高的化学动力学稳定性, 这一研究为超分子络合物在生物医学和制药方面的应用提供了一种新的可能。 


\section{Acknowledgements}

It might be difficult to write down a scientific article because you need tables, charts, graphs, characterizations to demonstrate. However, it must be easy to appreciate someone just by writing it down without any calculations. That is a thing I'd like to do here.

I always chitchat with my fellows, why time flies faster in the Netherlands? Four years just like a yesterday thing unless I hold my full thesis on hands. I am grateful that I can make my $\mathrm{PhD}$ life organized on time, well, without all family, friends, colleagues and supervisors' support, it would not be possible.

Foremost, my sincerely appreciation goes to my promotor, Prof.dr. Marcel Karperien, for kindly offering me this opportunity and freedom to conduct my $\mathrm{PhD}$ research in the Developmental BioEngineering group. I also appreciate your input on my final thesis correction. To my direct supervisor, Prof.dr. Piet Dijkstra, I don't know how to use words to express my appreciations, not only because of picking me up in the airport at first time came to the Netherlands, introducing me to all the registration procedures, offering me a free bike, taking a tour to the city, helping me moving home, encouraging me joining all the conferences and meetings but also because of your kind attitudes to guide me to the right directions of the research, professional input on our publications, patience on improving my English writing skills as well as introducing me for my future careers. I like working with you, because you never blame me jumping into your office and telling you the new creations I made. I think we may create very fascinating work anyway (we always believe that, $\odot$ ). In addition, Greatest thanks to Janny Dijkstra for joyful dinner time and wonderful trip together.

Moreover, I would like to thank Prof.dr.ir. W.E. Hennink, Prof.dr.ir. L.H. Koole, Dr. T.H. van Kuppevelt, Prof.dr.ir. J.W.M. Hilgenkamp, Prof.dr. J. Feijen, Prof.dr.ir. J. Huskens, Prof.dr. P.C.J.J. Passier and Prof.dr. D.B.F. Saris for taking place in the graduation committee.

Special appreciation goes to my Master supervisor, Prof.dr. Zhiyuan Zhong. You learned me well during master projects and you introduced me to this position. It means a lot to me and I'm appreciated your trust. 
I acknowledge my two paranymphs, Feb and Leilei Zhong, thanks very much for supporting me at this meaningful moment, we shared quite pleasant time together, not only dinners but also friendship. Feb, you are so young, smart, social and supportive, you opened my social circle, encouraged me to understand different cultures. Most importantly, you introduced me to the group of MERLN in Maastricht University as another post-doc that we can work together for the same project. I am so happy I knew you in the beginning of my life in Enschede and we can share more fun time together in the future. Leilie, you were the first Chinese friend I had in Enschede even you were not starting yet. We're sharing the office for four years, sharing countless times of dinners with Xiaobin and we are more like a family abroad. I witness the born and the care of Meilan, I am proud that I could be his exclusive aunt. I love him so much and I wish all the best to you and your family.

I would like to thank my students that helped me on accomplishing few chapters. Nadine and Christphor, you guys involved in the research at beginning of my $\mathrm{PhD}$ projects. Mike Geven, a talented student, not only because of your scientific views but also your kind personality, allow us to achieve the new discoveries in our new projects. I wish you all the best in your PhD research. Xandra, you are a special, precise and careful girl. Good luck with your new job and your life. In addition, good collaborations with Paula (from Span), Shaun, Caroline, Claudia (from Pisa, Italy), Lucia (from Pisa, Italy), Ivo Que (LUMC) and Yao made me got close to my goals. Thanks all for your contributions.

Too many colleagues, friends and family involved in these four years, I appreciate your help during the time, not only on scientific area but also social life. Jetse, Sieger Leilei and Jan, it was great luck to be an officemate with you. We shared quite some talks and discussions. Jean, Elahe, Niloofar, Jingwei, Parthiban, Mijke, Tim, Erik, Ivan and Paul thanks for being friends and sharing fun time together, great wishes for your future. Ingrid, thanks for your input for my graduation administration and also arrangements of group activities. Jacqueline, Sanne, Janine, Aart, Liliana, Jeroen, Janneke, Nicole, Jos, Giulia, Milou, Tom, Corina, Kannan, Maurice, Joao, it is a pleasure to be a colleague with you and thanks on helping me solve the biological problems. Marc Ankone, thanks for your guidance and advice concerning safety in the labs, although I broke lots of rules, you are still patient to tell me again and again. 
I am glad that during these years I joined two associations, PNUT and ACSSE. Because of this, I have met plenty of excellent $\mathrm{PhD}$ candidates and motherland friends. Thanks all for sharing great time together. It's hard to name them all, but I have all these beautiful memories in head. I specially appreciate all friends helped me to organize events when I was in PNUT and ACSSE. Nana, Mohammadreza, Adithya, David, Feb, Mihaela, Jonathan, Katarzyna, Anja, Janne, Joana and Burcu, thanks for great time and interesting events organized by PNUT. Xiaohua (小华), Junwen (珺文), Jianghan (姜涵), Luman (曼曼), Qibo (琦博), Yi Wang (王毅), Zhe Zhao (赵喆), Haoran (昊然) and few more volunteers, Peng Gao (高鹏), Lu Zhou (陆洲), Xiaokui Zhang (张小奎), Wenlong (文龙), Xingwu (醒悟), Yin Tao (陶寅), Quentin (东方), Lantian (蓝天), Ying Du (杜莹), Letian (乐天), Yuyu (玉玉), Zhoufang (方舟), Kenan (珂楠), Honglin (洪林), Minmin (敏敏), Xichen (曦晨) and all others, I appreciate all your contributions and wish you all the best. I also wish the new ACSSE board, Zhengchao Guo, Yuxin Zhou, Yunlong Yu and few new members have a great passion and contribute your time to make the life a bit colorful for Chinese students in Enschede.

Fortunately, I have the chance to express my appreciations to my future supervisors, Dr. Lorenzo Moroni and Aylvin Dias, thanks very much for taking me as your team, we together will make our new goal carried out sooner or later.

最后, 我想用自己国家的语言来感谢我的父母，兄长嫂及我的小林同学，以及 已逝的奶奶。

真心感谢你们的一直栽培, 信任爱护和挂念。我的所有一切经历和成果都是你 们从小教育, 陪伴的果实, 千言万语也无法感谢你们所做的一切。

愿健康和长寿时时围绕着父母和兄长嫂, 也希望我们可以经常团聚。

愿我的小林同学早日实现梦想。

愿奶奶安息。

我知道你们祝福我顺利毕业, 生活幸福。

11-12-2015

Rong / 荣

Enschede, the Netherlands 


\section{About the author}

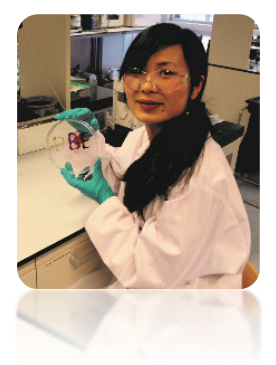

Rong Wang was born on February 1, 1984 in Suining, city of Xuzhou, a small town in Jiangsu province of China. Rong received her B.Sc. degree in the subject of Chemical Engineering and Technology at the Huaihai Institute of Technology in June, 2008. Starting from September, 2008, she continued her Master education in a major of Applied Chemistry at Soochow University, China. She was graduated in June, 2011with a Master dissertation entitled "Functional Polycarbonate Polymers for the Tissue Engineering" (supervisor: Prof. Zhiyuan Zhong, leader of BioMedical Polymers Laboratory). After graduation, she worked in an industry named EMS-CHEMIE (Suzhou) Ltd. for a half year and since February 1, 2012, she joined Developmental BioEngineering group in University of Twente as a PhD candidate under supervision of Prof. Piet Dijkstra and Prof. Marcel Karperien. Her main interests and focus are design and synthesis in-situ forming or called injectable biodegradable hydrogels based on natural or synthetic polymers for tissue engineering applications. Most of her work has been published in international journals and/or presented in international meetings. In July, 2013, she was awarded a Scholarships for summer school on Biomaterials and Regenerative Medicine, Italy. In March, 2015, she together with her supervisor Prof.dr. Piet were awarded a public award on 20 Seconds Science movie competition organized by MESA+ and MIRA faculties in University of Twente. During her stay in the Netherlands (2012-2016), She joined PhD network association in University of Twente (PNUT) and Chinese association in Enschede (ACSSE). 


\section{Publications}

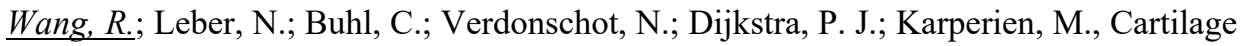
adhesive and mechanical properties of enzymatically crosslinked polysaccharide tyramine conjugate hydrogels. Polym. Adv. Technol. 2014, 25 (5), 568-574.

Wang, R.; Geven, M.; Dijkstra, P. J.; Martens, P.; Karperien, M., Hydrogels by supramolecular crosslinking of terpyridine end group functionalized 8-arm poly(ethylene glycol). Soft Matter 2014, 10 (37), 7328-7336.

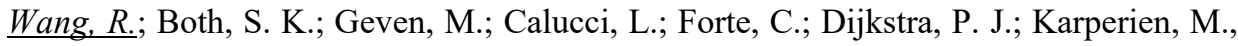
Kinetically stable metal ligand charge transfer complexes as crosslinks in nanogels/hydrogels: Physical properties and cytotoxicity. Acta Biomater. 2015, 26, 136144.

$\underline{\text { Wang } R .}$, Que I., Y Fu, Both S., Chan A., Dijkstra P. J., Karperien, M., Non-invasive near-infrared fluorescence in vivo imaging of biodegradable enzymatically crosslinked polysaccharide hydrogels. 2015. In preparation.

$\underline{\operatorname{Wang} R}$, Huang X. B., Dijkstra P. J., Karperien, M., Enzymatic co-crosslinking of starshaped poly(ethylene glycol) tyramine and hyaluronic acid tyramine conjugates provides elastic biocompatible and biodegradable hydrogels. 2015. In preparation.

Wang, R.; Chen, W.; Meng, F.; Cheng, R.; Deng, C.; Feijen, J.; Zhong, Z., Unprecedented Access to Functional Biodegradable Polymers and Coatings. Macromolecules 2011, 44 (15), 6009-6016.

Chen, W.; Yang, H.; Wang, R.; Cheng, R.; Meng, F.; Wei, W.; Zhong, Z., Versatile Synthesis of Functional Biodegradable Polymers by Combining Ring-Opening Polymerization and Postpolymerization Modification via Michael-Type Addition Reaction. Macromolecules 2010, 43 (1), 201-207.

Deng, C.; Wang, R.; Chen, W.; Meng, F.-h.; Cheng, R.; Zhong, Z.-y., Design and synthesis of rapidly photo-crosslinkable bioactive biodegradable hydrogels. Acta Polymerica Sinica 20135, 695-704. 


\section{Patents}

陈维, 王荣, 孟风华, 钟志远, 含有丙烯酸酯类功能基团的环碳酸酯单体及其制备和应用, 专利申请号：200910181912.2.

Wei Chen, Fenghua Meng, Rong Wang, and Zhiyuan Zhong, A method of making a polymer preferably an (alkyl)acryloyl polycarbonate PCT/EP2009/008859.

Zhiyuan Zhong, Fenghua Meng, Rong Wang and J. Feijen, Vinyl sulfone functionalized biodegradable polymers, PCT/EP2011/061256. 